\title{
Kohlenstoff- und Stickstofftransport in temperaten Laubbäumen und ihren Mykorrhizen
}

Transport of carbon and nitrogen in temperate broadleaf tree species and their associated mycorrhizas

Dissertation zur Erlangung des Dr. forest an der

Forstwissenschaftlichen Fakultät der Georg-August-Universität Göttingen

vorgelegt von

Michaela Rath

aus Fuldatal

Göttingen, im Juni 2015 
1. Gutachter: Prof. Andrea Polle

2. Gutachter: Prof. Stefan Scheu

Tag der mündlichen Prüfung: 16. Juli 2015 


\section{Zusammenfassung}

1.Kapitel Die einheimische Laubbaumarten Buche, Esche, Linde und Ahorn und ihre Mykorrhiza

1.2 Stofftransport in Bäumen 12

1.3 Anwendung von Isotopen in Tracerexperimenten 13

1.4 Ziele dieser Arbeit 14

$\begin{array}{ll}1.5 \text { Literatur } & 16\end{array}$

2. Kapitel: Stickstoffallokation in den Feinwurzeln der Buche (Fagus sylvatica) in Rein$\begin{array}{lr}\text { und Mischbeständen im Nationalpark Hainich } & 18\end{array}$

$\begin{array}{lll}2.1 & \text { Einleitung } & 18\end{array}$

2.2 Material \& Methoden 20

$\begin{array}{ll}\text { 2.2.1 Versuchsaufbau } & 20\end{array}$

$\begin{array}{ll}\text { 2.2.2 Probenbearbeitung } & 25\end{array}$

2.2.3 Bestimmung der Isotopensignatur 25

$\begin{array}{ll}\text { 2.2.4 Mykorrhizaanalyse } & 26\end{array}$

$\begin{array}{ll}\text { 2.2.4.1 Mykorrhizierungs- und Vitalitätsrate } & 27\end{array}$

$\begin{array}{ll}\text { 2.2.5 Diversitätsindex Shannon-Index } & 27\end{array}$

$\begin{array}{ll}\text { 2.2.6 Statistik } & 27\end{array}$

$\begin{array}{lll}2.3 & \text { Ergebnisse } & 28\end{array}$

$\begin{array}{ll}2.3 .1 \text { Biomasse } & 28\end{array}$

2.3.2 ${ }^{15} \mathrm{~N}$-Allokation in den Wurzeln und im Boden 30

2.3.3 Charakterisierung der Mykorrhizagesellschaft 33

2.4 Diskussion 36

$\begin{array}{lll}2.5 & \text { Literatur } & 38\end{array}$

3.Kapitel: C- und N-Fluss in Buche (Fagus sylvatica) und Esche (Fraxinus excelsior) von den Blättern in mykorrhizierte Wurzelspitzen 40

$\begin{array}{lll}3.1 & \text { Einleitung } & 40\end{array}$

$\begin{array}{lll}3.2 & \text { Material \& Methoden } & 43\end{array}$

3.2.1 Untersuchungsgebiet und Auswahl der Pflanzen 43 
3.2.2.1 Kohlenstoffmarkierung 45

3.2.2.2 Stickstoffmarkierung $\quad 49$

3.2.3 Ernte 52

3.2.4 Mykorrhizauntersuchung 52

3.2.4.1 Ektomykorrhiza an Buche 52

3.2.4.2 Arbuskuläre Mykorrhiza an Esche 53

3.2.5 Isotopenbestimmung $\quad 54$

3.2.6 Statistik 56

$\begin{array}{lll}3.3 & \text { Ergebnisse } & 57\end{array}$

$\begin{array}{ll}3.3 .1 \text { Isotopenmarkierung } & 57\end{array}$

3.3.1.1 Kohlenstoffmarkierung in Rinde, Feinwurzel und vitalen Wurzelspitzen von $\begin{array}{ll}\text { Buche und Esche } & 57\end{array}$

3.3.1.2 Stickstoffmarkierung in Rinde, Feinwurzel und vitalen Wurzelspitzen von $\begin{array}{ll}\text { Buche und Esche } & 61\end{array}$

3.4 Diskussion 66

$\begin{array}{ll}\text { 3.5 Literatur } & 69\end{array}$

4. Kapitel: Vitalitätsuntersuchungen an Wurzelspitzen von Buche (Fagus sylvatica) und Esche (Fraxinus excelsior)

$\begin{array}{ll}\text { 4.1 Einleitung } & 71\end{array}$

4.2 Material \& Methoden $\quad 74$

4.2.1 Optische Ansprache der Wurzelspitzen $\quad 74$

4.2.2 Einbettung der Wurzelspitzen zur Erstellung anatomischer Querschnitte $\quad 75$

4.2.3 Vitalitätsbestimmung der Wurzelspitzen an Hand von TTC-Tests 76

4.2.4 Analyse der ${ }^{13} \mathrm{C}$ - und ${ }^{15} \mathrm{~N}$-Akkumulation in Wurzelspitzen 77

$\begin{array}{ll}\text { 4.2.5 Statistik } & 77\end{array}$

$\begin{array}{ll}4.3 \text { Ergebnisse } & 78\end{array}$

4.3.1 Analyse der morphologischen Merkmale an Hand von Oberflächenaufnahmen und anatomischen Querschnitte 78

4.3.2 TTC-Tests an vitalen und trockenen Wurzelspitzen 80 
4.3.3 ${ }^{13} \mathrm{C}$ - und ${ }^{15} \mathrm{~N}$ - Transport in trockene Wurzelspitzen 81

$\begin{array}{ll}4.4 \text { Diskussion } & 86\end{array}$

$\begin{array}{ll}4.5 \text { Literatur } & 88\end{array}$

5. Kapitel: 213C- und 214C- Doppelmarkierung zur Analyse des Transportes von Kohlenstoff in die Rhizosphäre einheimischer Laubbaumarten

$\begin{array}{lll}5.1 & \text { Einleitung } & 90\end{array}$

5.2 Material \& Methoden 92

$\begin{array}{ll}\text { 5.2.1 Vorbereitung } & 92\end{array}$

5.2.2 13CO2- und 14CO2-Begasung 94

$\begin{array}{ll}\text { 5.2.3 Ernte } & 97\end{array}$

$\begin{array}{ll}\text { 5.2.4 Mykorrhiza } & 98\end{array}$

$\begin{array}{ll}\text { 5.2.4.1 Probennahme von Wurzelspitzen } & 98\end{array}$

$\begin{array}{ll}5.2 .5 \text { Isotopenanalyse } & 101\end{array}$

5.2.5.1 13C und 14C in verschiedenen Kompartimenten 101

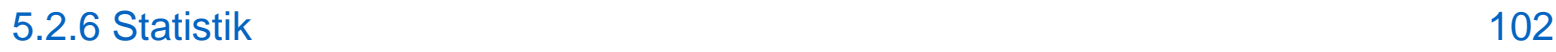

$\begin{array}{ll}5.3 \text { Ergebnisse } & 103\end{array}$

$\begin{array}{ll}\text { 5.3.1 Biometrische Analysen } & 103\end{array}$

5.3.2 Anreicherung von altem und neuem Kohlenstoff in Pflanzen 106

5.3.2.1 13C und 14C in oberirdischen Pflanzenkompartimenten 106

5.3.2.2 13C und 14C in unterirdischen Pflanzenkompartimenten 109

5.3.2.3 13C und 14C in Rhizosphärenboden und Mischboden 112

$\begin{array}{ll}5.4 \text { Diskussion } & 115\end{array}$

$\begin{array}{ll}5.5 \text { Literatur } & 117\end{array}$

$\begin{array}{ll}\text { 6. Synopsis } & 119\end{array}$

$\begin{array}{ll}\text { 7. Summary } & 123\end{array}$

$\begin{array}{ll}\text { 8. Anhang } & 125\end{array}$

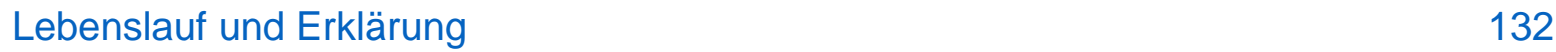

$\begin{array}{ll}\text { Danksagung } & 134\end{array}$ 
Abkürzungsverzeichnis (mit Chemikalien und Einheiten)

\begin{tabular}{|c|c|}
\hline 'E & east (Ost) \\
\hline$N$ & north ( Nord) \\
\hline$\partial$ & Delta \\
\hline${ }^{\circ} \mathrm{C}$ & Grad Celsius \\
\hline$\mu \mathrm{g}$ & Mikrogramm \\
\hline$\mu \mathrm{l}$ & Mikroliter \\
\hline$\%$ & Prozent \\
\hline$\%$ & Promille \\
\hline Abb. & Abbildung \\
\hline Abs & Absorption \\
\hline AG & Aktiengesellschaft \\
\hline$A G$ & Arbeitsgruppe \\
\hline ANOVA & Analyse of Variance \\
\hline bzw. & beziehungsweise \\
\hline C & Kohlenstoff \\
\hline $\mathrm{Ca}\left({ }^{15} \mathrm{NO}_{3}\right)_{2}$ & ${ }^{15} \mathrm{~N}$ - markiertes Calciumnitrat \\
\hline $\mathrm{ccm}$ & Kubikzentimeter \\
\hline $\mathrm{cm}$ & Zentimeter \\
\hline $\mathrm{CO}_{2}$ & Kohlendioxid \\
\hline d.h. & das heißt \\
\hline dest. & destilliertes (Wasser) \\
\hline DL (DI) & Diversitätslevel \\
\hline dpm & decay per minute; Zerfall pro Minute \\
\hline et al. & et alii (lat. und andere) \\
\hline$g$ & Gramm \\
\hline $\mathrm{GmbH}$ & Gesellschaft mit beschränkter Haftung \\
\hline $\mathrm{h}$ & Stunde \\
\hline $\mathrm{H}_{2} \mathrm{SO}_{4}$ & Schwefelsäure \\
\hline ha & Hektar \\
\hline HT & Hochtemperatur \\
\hline ITS-Region & Internal Transcribed Spacer Region \\
\hline $\mathrm{KH}_{2} \mathrm{PO}_{4}$ & Kaliumdihydrogenphosphat \\
\hline
\end{tabular}




\begin{tabular}{ll} 
KOH & Kalilauge \\
KOSI & Kompetenzzentrum für Stabile Isotope \\
LARI & Liter \\
$\mathrm{m}$ & Labor für Radioaktive Isotope \\
$\mathrm{M}$ & Meter \\
$\mathrm{mg}$ & Molar \\
$\mathrm{min}$ & Milligramm \\
$\mathrm{ml}$ & Minute \\
$\mathrm{mm}$ & Milliliter \\
$\mathrm{mol}$ & Meter \\
$\mathrm{MWS}$ & Mol \\
$\mathrm{N}$ & mykorrhizierte Wurzelspitzen \\
$\mathrm{Na} 2 \mathrm{CO}$ & Stickstoff \\
$\mathrm{NCBI}$ & Natriumcarbonat \\
$\mathrm{nm}$ & Nationales Zentrum für Biotechnologische Information \\
$\mathrm{rpm}$ & Nanometer \\
$\mathrm{TTC}$ & round per minute (Umdrehung pro Minute) \\
$\mathrm{TWS}$ & Triophenyltetrazolium \\
UK & trockene Wurzelspitzen \\
UNITE & United Kingdom \\
USA & Datenbank zur Identifizierung von Ektomykorrhiza \\
V & United States of America \\
vgl. & Volt \\
V-PDB & vergleiche \\
VWS & PeeDee-Formation \\
$\mathrm{W}$ & vitale Wurzelspitzen \\
z.B. & Watt \\
& zum Beispiel \\
\hline
\end{tabular}




\section{Zusammenfassung}

Die einheimischen Laubbäume sind mit Mykorrhizen vergesellschaftet. Diese Symbiose dient der gegenseitigen Nährstoffversorgung von Pflanze und Pilz. Die in Europa dominante Buche (Fagus sylvatica) ist mit Ektomykorrhizen vergesellschaftet und tritt in Deutschland sowohl in Reinbeständen als auch in Vergesellschaftung mit weiteren Laubbaumarten wie Linde, Esche und Ahorn auf. In der vorliegenden Arbeit wurde der Stofftransport zweier wichtiger Pflanzennährstoffe, Kohlenstoff und Stickstoff, von der Pflanze in die Rhizosphäre untersucht. Dabei wurde die Buche mit weiteren Laubbäumen verglichen und der Aspekt unterschiedlicher Mykorrhizaformen an den Baumarten beleuchtet. Dazu wurden mit Anwendung der Isotopentechnik als Tracer der Stoffe verschiedene Markierungsexperimente durchgeführt.

Die Buche ist mit Ektomykorrhizapilzen vergesellschaftet und wurde in der Untersuchung mit der endomykorrhizierten Esche verglichen. Daneben wurden die Untersuchungen der vorliegenden Arbeit mit dem - in Bezug auf Isotopentechnikbisher in geringerem Umfang untersuchten Bergahorn und der Winterlinde erweitert, um den Einblick in den Transport weiter zu vertiefen. Bei der Auswahl der beiden Baumarten waren zudem auch die Endomykorrhiza des Bergahornes und die Ektomykorrhiza der Winterlinde von Bedeutung. Um den Fokus auf die beiden Baumarten Buche und Esche in Bezug auf physiologische Aspekte zu vertiefen, wurde eine Vitalitätsuntersuchung durchgeführt.

Um den Transport von Kohlenstoff und Stickstoff in den einheimischen Baumarten und ihrer assoziierten Mykorrhiza zu analysieren, wurden verschiedene Markierungsexperimente durchgeführt.

In einem Freilandexperiment wurde die Aufnahme von Stickstoff in Buchenwurzeln sowohl im Rein- als auch im Mischbestand untersucht. Dazu wurde der Boden mit ${ }^{15} \mathrm{~N}$ markiert und die Aufnahme über einen Zeitraum von 8 Tagen analysiert. Dabei zeigte sich sowohl im Buchenreinbestand als auch im Buchenmischbestand eine starke Anreicherung von ${ }^{15} \mathrm{~N}$ in den Feinwurzeln zu Beginn mit verbleibendem konstantem Niveau über eine Woche. Dabei zeigte der Reinbestand eine höhere Stickstoffanreicherung in den Feinwurzeln als der Mischbestand. Dies wird als fehlende Konkurrenz um Stickstoff durch andere Baumarten interpretiert. 
Weiter wurde ein Freilandmarkierungsexperiment an Buche und Esche durchgeführt. Dabei wurde der Stofftransport innerhalb der Pflanze zur Rhizosphäre an zwei verschieden mykorrhizierten Baumarten untersucht, um den Einfluss der Mykorrhiza auf C- und N-Transport zu untersuchen. Die Bäume wurden dabei über die Blätter für drei Tage mit ${ }^{15} \mathrm{~N}$ und im Anschluss für 5 Stunden mit ${ }^{13} \mathrm{CO}_{2}$ markiert. Der Transport von $\mathrm{C}$ und $\mathrm{N}$ wurde über einen Zeitraum von 60 Tagen analysiert. Es zeigte sich, dass die endomykorrhizierte Esche sowohl eine höhere C- als auch N-Akkumulation in ihren Feinwurzeln und Wurzelspitzen aufwies als die ektomykorrhizierte Buche.

Um die Frage zu beantworten, ob und inwiefern der Stofftransport in der Pflanze aus physiologischer Sicht im Zusammenhang mit dem Vitalitätszustand der Wurzeln, insbesondere der Feinwurzeln, steht, wurde eine Vitalitätsuntersuchung mit einer Isotopenmarkierung mit ${ }^{15} \mathrm{~N}$ kombiniert. Dazu wurde an den beiden untersuchten Baumarten Buche und Esche die Methode der visuellen Ansprache von Wurzeln überprüft. Dabei wurden Wurzelspitzen an Hand von TTC-Tests auf ihren Vitalitätszustand hin überprüft. Aus dem Vergleich von vitalen und trockenen Wurzelspitzen ergab sich, dass die Methode der visuellen Ansprache bei der ektomykorrhizierten Buche verlässlich anwendbar ist, bei der endomykorrhizierten Esche jedoch nicht, da die trockenen Wurzelspitzen von Esche nahezu eine der vitalen Buchenwurzelspitzen vergleichbare Absorption reduzierten Farbstoffes zeigten. In Bezug auf die Isotopenmarkierung zeigte sich, dass in den trockenen Wurzelspitzen - im Gegensatz zur Buche- sowohl eine ${ }^{15} \mathrm{~N}$ als auch ${ }^{13} \mathrm{C}$ Akkumulation stattfand.

Die Frage, ob die Rhizosphäre durch langfristige oder kurzfristige C-Ressourcen von der Pflanze gespeist wird, wurde an Hand eines Gewächshausexperimentes an Bergahorn, Buche und Winterlinde untersucht. Dabei wurde eine langfristige ${ }^{13} \mathrm{CO}_{2}$ Begasung über zwei Vegetationsperioden mit einer kurzfristigen ${ }^{14} \mathrm{CO}_{2}$ Pulsbegasung kombiniert. Es zeigte sich dabei in den drei Baumarten, dass sowohl langfristiges als auch kurzfristiges $C$ von den Blättern in die Rhizosphäre transportiert wird. Daneben trat eine deutliche Anreicherung von beiden Isotopen in allen Pflanzenorganen über einen kurzen Zeitraum auf, die dann in der folgenden Vegetationsperiode absank. Es zeigte sich, dass über die kurzfristige Speicherung von Kohlenstoff hinaus, auch eine - wenn auch deutlich geringere- längerfristige Speicherung erfolgt. Dies wurde sowohl beim Kohlenstoff, der über zwei 
Vegetationsperiode gegeben wurde, als auch bei der kurzfristigen Pulsmarkierung beobachtet.

Zusammenfassend lässt sich für die vorliegende Arbeit festhalten, dass in allen untersuchten einheimischen Laubbaumarten der Stofftransport von den Blättern zu den Wurzeln nachgewiesen wurde und teilweise Unterschiede in den Transportstrategien der Baumarten auftreten. Besonders deutlich wurde dies in Bezug auf die ektomykorrhizierte Buche, die im Vergleich zur endomykorrhizierten Esche mehr Kohlenstoff und Stickstoff in der Rinde akkumulierte, in Bezug auf die unterirdischen Pflanzenorgane jedoch geringere Anreicherungen zeigte. 


\section{Kapitel: Einleitung}

1.1 Die einheimische Laubbaumarten Buche, Esche, Linde und Ahorn und ihre Mykorrhiza

$\mathrm{Zu}$ den einheimischen Laubbaumarten gehören Buche (Fagus sylvatica), Ahorn (Acer spec.), Esche (Fraxinus excelsor) und Linde (Tilia spec.). Die Buche ist in Mitteleuropa die konkurrenzstärkste Baumart (Ellenberg, 1996) und dominiert auf Grund ihrer hohen Standortamplitude viele Waldbestände (Röhrig \& Bartsch, 1992). Sie tritt auf mittel- bis tiefgründigen, kalkhaltigen Boden mit guter Nährstoffversorgung vorzugsweise auf (Röhrig \& Bartsch, 1992). Sie ist wie ihre vergesellschafteten Laubbaumarten Esche, Ahorn und Linde, die zu den Edellaubbäumen zählen, eine schattentolerante Baumart (Ellenberg, 1996; Röhrig \& Bartsch, 1992). Im Vergleich zu den genannten Edellaubbäumen zeigt die Buche höhere Toleranz gegen Bodensäure, aber niedrigere in Bezug auf Bodenfeuchte (Röhrig \& Bartsch, 1992). Das Auftreten der Buche in Deutschland besitzt nach der dritten Bundeswaldinventur einen Anteil von 15,4 \% und die Baumarten Ahorn, Linde und Esche, die in der Inventur zu den langlebigen Laubbaumarten gezählt werden, besitzen in Deutschland einen Anteil von zusammen 7,1\% (www.bundeswaldinventur.de). Gemeinsam treten die Baumarten in europäischen Waldgesellschaften auf, in denen die Esche als Charakterart und Linde und Ahorn als typische Begleitbaumart bezeichnet werden (Stinglwagner et al., 2005).

Die meisten höheren Pflanzen leben in einer Symbiose mit Pilzen (Smith \& Read, 1997). Bis zu 90\% aller Landpflanzen sind mit Pilzen aus 6000 unterschiedlichen Arten fakultativ oder obligat in Form der Mykorrhiza vergesellschaftet. Die Mykorrhiza dient dem gegenseitigen Austausch von Nährstoffen zwischen Pflanzenwurzel und Pilz (Simard et al., 1997). Dabei versorgt der Pilz die Pflanze beispielsweise mit Stickstoff (Smith \& Read, 1997). Der Anteil des photosynthetisch durch die Pflanze erzeugten Zuckers, der an den Pilz weitergegeben wird, ist mit 20\% nicht unerheblich (Jackobsen \& Rosendahl, 1990). Insbesondere bei schlechter Nährstoffversorgung wie in der borealen und temperaten Zone ist die Mykorrhiza von großer Bedeutung (Högberg et al., 1996; Tamm, 1991). Der Pilz ist weiterhin in der Lage, gegenüber der Pflanze eine Schutzfunktion einzunehmen, beispielsweise gegen Trockenheit (Shi et al., 2002) oder Schwermetalle (Schutzendübel \& Polle, 2002). Unter den verschiedenen Mykorrhizaformen (Smith \& Read, 1997) ist die arbuskuläre 
Mykorrhiza mit 70 - 90\% am weitesten verbreitet ist (Fitter, 2005; Smith \& Read, 2008) ist. Sie findet sich in temperaten Wäldern eher selten, kommt aber unter anderem an der hier untersuchten Esche und Ahorn vor (Lang et al., 2011; Seven \& Polle, 2014). Die Ektomykorrhiza kommt in borealen und temperaten Wäldern häufig vor und findet sich an Buche und Linde (Lang et al., 2011; Pena et al., 2013).

Die in Deutschland als dominante Baumart auftretende Buche (Ellenberg, 2009) ist nahezu zu 100\% mykorrhiziert (Pena et al., 2010; Lang et al. 2011). Die Pilzhyphen der Ektomykorrhiza bilden um die Wurzelspitze einen Mantel und bilden im Inneren der Wurzelspitze zwischen den Zellen das sogenannte Hartig'sche Netz, dringen aber nicht ins Wurzelinnere ein. Hyphenmantel und Hartig'sches Netz besitzen unterschiedliche Funktionen (Kottke \& Oberwinkler, 1986). Durch das Hyphengewebe wird die transportaktive Oberfläche für Nährstoffe und Wasser vergrößert. Durch den geringeren Durchmesser der Pilzhyphen im Vergleich zu den Wurzelhaaren, die sie ersetzen, können im Boden feinere Poren erschlossen werden, wodurch eine weitere Oberflächenvergrößerung von physiologisch aktivem Gewebe bewirkt wird. Die charakteristischen Merkmale des Hyphenmantels der Ektomykorrhiza sind Farbe, Oberflächenbeschaffenheit, Verzweigung sowie die Erkennbarkeit von Hyphen, die ins Bodenreich ausstrahlen (Agerer, 1987 - 2006).

Die arbuskuläre Mykorrhiza, die mit der Esche assoziiert ist, bildet wie die Ektomykorrhia Hyphen aus, aber im Vergleich zur Ektomykorrhiza ist die arbuskuläre Mykorrhiza in der Lage innerhalb der Wurzelzellen zu wachsen und bildet dort charakteristische Arbuskel aus (Smith \& Read, 1997). Die arbuskuläre Mykorrhiza verbindet Pflanzen über ein Netzwerk des Pilzes, das aus bis zu 100 Meter Hyphen pro $\mathrm{m}^{2}$ Boden bestehen kann (Miller et al., 1995) und auf den Transport von Wasser und Phosphor spezialisiert ist (Finlay, 2008). Eine Spezialisierung von Ekto- und Endomykorrhiza auf Nährelemente wurde in einer Untersuchung von Seven \& Polle (2014) beobachtet, bei der beispielsweise Ektomykorrhiza eine bedeutende Rolle in der Schwefel- und Phosphorernährung von Pflanzen spielte, wohingegen die Endomykorrhiza hohe Kaliumkonzentrationen aufwies.

1.2 Stoftransport in Bäumen

Bäume benötigen zur Aufrechterhaltung ihres Stoffwechsels und ihrer Biomasseproduktion Nährstoffe. Aus atmosphärischem Kohledioxid ( $\left.\mathrm{CO}_{2}\right)$ produzieren Bäume in der photosyntetisch aktiven oberirdischen Biomasse mit Hilfe 
von Lichtenergie Kohlenhydrate. Vom Produktionsort ("source") wird der Kohlenstoff zu den Verbrauchorten ("sink") transportiert und wird dort akkumuliert oder weiter metabolisiert. "Sinks" sind zu einem großen Teil die Wurzeln des Baumes selbst jedoch auch mit Wurzeln assoziierte Pilze und Bakterien. Bis zu $20 \%$ des photosynthetisch erzeguten Zuckers werden zur Mykorrhiza transportiert (Söderström, 1992). Der Transport von Kohlenstoff erfolgt von somit von den Blätter durch den Baum in Richtung der unterirdischen Biomasse. Dem gegenüber erfolgt der Transport von Stickstoff hauptsächlich in umgekehrter Richtung - von der Wurzel aus der Bodenlösung aufgenommen, wird dieser als lon in Form von Nitrat oder Ammonium, durch die Pflanze zu den Verbrauchsorten transportiert. Die Bedeutung des Stickstofftransportes durch die Mykorrhiza kommt insbesondere dort zum Tragen, wo Stickstoff der limitierende Faktor für das Pflanzenwachstum ist (Read, 1992), der durch die Mykorrhiza pflanzenverfügbar gemacht wird.

\subsection{Anwendung von Isotopen in Tracerexperimenten}

Die wichtigen Pflanzennährelemente $\mathrm{C}$ und $\mathrm{N}$ kommen in Form verschiedener Isotope vor. Die Isotope besitzen die gleiche Anzahl an Protonen und Elektronen, unterscheiden sich jedoch in der Anzahl ihrer Neutronen und dadurch in ihrer Masse. Das Kohlenstoffatom kommt zum Beispiel in der Luft zu 98,9 \% aus ${ }^{12} \mathrm{C}, 1,1 \%{ }^{13} \mathrm{C}$ und $10^{-10} \%{ }^{14} \mathrm{C}$ vor. Bei den beiden leichteren Isotopen ${ }^{12} \mathrm{C}$ und ${ }^{13} \mathrm{C}$ handelt es sich um stabile Isotope, während das schwerste Kohlenstoffisotop ${ }^{14} \mathrm{C}$ radioaktiv ist. ${ }^{12} \mathrm{C}$ wird auch als natürlicher Kohlenstoff bezeichnet. Das Stickstoffatom besteht aus zwei stabilen Isotopen zu 99,63\% aus dem leichten ${ }^{14} \mathrm{~N}$ und zu 0,366 \& aus dem schweren ${ }^{15} \mathrm{~N}$.

Der Einsatz stabiler Isotope in ökologischen und biogeochemischen Untersuchungen hat seit den Anfängen in den 1980er Jahren rapide zugenommen, da an Hand dieser Tracer die Untersuchung von sink-source-Beziehungen und Stofftransport detektierbar gemacht werden konnte (Peterson \& Fry, 1987). Insbesondere die pflanzenphysiologisch bedeutsamen Elemente Kohlenstoff, Stickstoff, Wasserstoff und Sauerstoff werden häufig in Untersuchungen eingesetzt. Sie verfügen alle über mehrere Isotope und ihre spezifischen Signaturen können präzise mit Hilfe der Massenspektrometrie gemessen werden (Peterson \& Fry, 1987). Bei den natürlichen Signaturen der schweren relativ zu den leichten Isotopen pflanzlicher Gewebe spricht 
man von "natural abundance“. Mit der Zugabe erhöhter Konzentrationen eines bestimmten Isotopes oberhalb der "natural abundance“ ist es möglich, Stofftransportprozesse nachzuweisen. Diese Markierungsexperimente beziehen sich je nach zeitlichem Verlauf auf Kurzzeituntersuchungen oder auf Langzeitexpositionen. Durch diese Untersuchungen ist es zum Beispiel möglich, im Freiland den Transport von ${ }^{13} \mathrm{C}$ innerhalb des Pflanzen-Boden-Systems (Epron et al., 2011) oder den des stabilen Stickstoffisotopes ${ }^{15} \mathrm{~N}$ zwischen mykorrhizierten Pflanzen (He et al., 2009) zu untersuchen. Die Methoden der Gaschromatographie und der Massenspektrometrie machen daneben auch die gleichzeitige Untersuchung verschiedener Isotopensignaturen durch in einem System möglich, wie zum Beispiel Aminosäureflüsse in Pflanzen-Mikroben-Vergesellschaftungen (Molero et al., 2011).

\subsection{Ziele dieser Arbeit}

In der vorliegenden Arbeit sollte der Transport von Stickstoff und Kohlenstoff in verschiedenen Baumarten untersucht werden, wozu verschiedene Experimente durchgeführt wurden. Der Fokus lag dabei auf dem Transport zu den Feinwurzeln und der angrenzenden Mykorrhiza sowie der Akkumulation in der Rhizosphäre.

Die Freilanduntersuchungen dieser Arbeit wurden im Nationalpark Hainich, einem von Norden nach Süden verlaufenden Höhenzug in Thüringen, in der Nähe der Stadt Mühlhausen, durchgeführt. Der Hainich als Europas größtes zusammenhängendes Laubwaldgebiet beherbergt auf seinen ca. 15000 Hektar Fläche zum Teil bis zu 14 verschiedene Laubbaumarten und gehört daher zu den baumartenreichsten Gebieten Mitteleuropas. Die vorliegenden Freilanduntersuchungen wurden in ausgewählten Bereichen wie der "Lichten Haardt" und auf den Flächen der ersten Forschungskohorte des GRK 1086, die auf Grund ihrer Baumartendiversität ausgewählt wurden, durchgeführt. Zu den untersuchten Baumarten des Hainichs gehören hier insbesondere die Buche (Fagus sylvatica), die mit Ahorn (Acer spec.), Esche (Fraxinus excelsior) und Linde (Tilia spec.) vergesellschaftet ist. Neben den Freilanduntersuchungen an Buche und Esche wurden an Linde, Buche und Ahorn auch Gewächshausversuche durchgeführt, in denen die Bäume in originalem Boden aus dem Hainich kultiviert wurden.

In der vorliegenden Arbeit sollen folgende Fragen beantwortet werden: 
1. Gibt es Unterschiede in der Stickstoffallokation in den Feinwurzeln der Buche in Bezug auf ihr Auftreten in Mono- und Mischbeständen?

Um die Bedeutung der Diversität für die Stickstoffversorgung von Buchenwurzeln zu untersuchen, wurde in einem Freilandexperiment im Hainich eine ${ }^{15} \mathrm{~N}$-Markierung der Feinwurzeln über den Boden durchgeführt. Dabei wurden Buchenreinbestände und Buchenbestände mit Einmischung von Ahorn, Esche, Hainbuche und Linde analysiert und miteinander verglichen. Die Stickstoffaufnahme der Buchenfeinwurzeln wurde dabei über einen kurzen Zeitraum von 8 Tagen untersucht.

2. Unterscheiden sich verschieden mykorrhizierte einheimische Laubbaumarten im Stofftransport?

Um die Untersuchung der Stickstoffaufnahme in den Buchenwurzeln zu erweitern, wurde ein Markierungsexperiment im Freiland durchgeführt, das neben der ektomykorrhizierten Buche die endomykorrhizierte Esche einbezog. An Hand einer Kombination von ${ }^{13} \mathrm{C}$ - und ${ }^{15} \mathrm{~N}$-Markierung wurde dabei der Transport zweier wichtiger Pflanzennährstoffe von den Blättern in die Wurzelspitzen über einen Zeitraum von 60 Tagen analysiert.

3. Erfolgt die Speisung die Rhizosphäre mit Kohlenstoff in einheimischen Laubbäumen aus kurzfristigen oder längerfristigen Ressourcen?

Um den Kohlenstofftransport genauer beurteilen zu können, wurde eine Doppelmarkierung mit ${ }^{13} \mathrm{CO}_{2}$ und ${ }^{14} \mathrm{CO}_{2}$ im Gewächshaus durchgeführt. In der Doppelmarkierung wurde über einen Zeitraum von zwei Vegetationsperioden eine Langzeitmarkierung mit einer Kurzzeitmarkierung kombiniert. Dabei sollten auch eventuelle Unterschiede zwischen einheimischen Laubbaumarten analysiert werden, sodass das Experiment neben der Buche an Linde und Ahorn durchgeführt wurde.

\section{Lässt sich die Vitalität von Wurzelspitzen visuell sicher bestimmen?}

Die Stoffanreicherung in Pflanzenkompartimenten hängt vom physiologischen Zustand ab. In einer Untersuchung zur Vitalitätsbestimmung von Wurzelspitzen an Hand von TTC-Tests wurde analysiert, ob sich Buchen- und Eschenwurzeln visuell verlässlich im Hinblick auf ihren Vitalitätszustand ansprechen lassen. 


\subsection{Literatur}

Agerer R (1987- 2006) Colour Atlas of Ectomycorrhizae. Einhorn Verlag, Schwäbisch Gmünd

Ellenberg H (1996) Vegetation Mitteleuropas mit den Alpen: in ökologischer, dynamischer und historischer Sicht. Eugen Ulmer Verlag, Stuttgart, 5. Auflage

Ellenberg H, Strutt GK (2009) Vegetation ecology of Central Europe, Cambridge University Press

Epron D, Ngao J, Dannoura M, Bakker MR, Zeller B, Bazot S, Bosc A, Plain C ,Lata JC, Priault P, Barthes L, Loustan D (2011) Seasonal variations of belowground carbon transfer assessed by in situ ${ }^{13} \mathrm{CO}_{2}$ pulse labelling of trees. Biogeosciences 8 : 1153- 1168

Finlay RD (2008) Ecological aspects of mycorrhizal symbiosis with special emphasison the functional diversity of interactions involving the extraradical mycelium. J Exp Bot 59: 1115-1126

Fitter A (2005) Darkness visible: Reflections on underground ecology. Journal of Ecology 93:231-243

He X, Minggang X, Guo YQ, Jianbin Z (2009) Use of ${ }^{15} \mathrm{~N}$ stable Isotope to quantify nitrogen transfer between mycorrhizal plants. J Plant Ecol Vol. 2 (3): 107-118

Högberg P, Högbom L, Schinkel H, Högberg M, Johannisson C, Wallmark H (1996) ${ }^{15} \mathrm{~N}$ abundance of surface soil, roots and mycorrhizas in profiles of European forest soils. Oecologia 108: 207-214

Jackobsen I, Rosendahl, L (1990) Carbon flow into soil and external hyphae from roots of mycorrhizal cucumber plants. New Phytol 115:77-83

Kottke I, Oberwinkler F(1986) Mycorrhiza of forest trees- structure and function. Trees 1:1-24

Lang C, Seven J, Polle A (2011) Host preferences and differential contributions of deciduous tree species mycorrhizal species richness in a mixed Central European forest. Mycorrhiza 21: 297- 308

Miller RM, Reinhardt DR, Jastrow JD (1995) External hyphal production of vesiculararbuscular mycorrhizal fungi in pasture and tallgrass prairie communities. Oecologia 103:17-23

Molero G, Aranjuelo I, Teixidor P, Araus JL, Nogues S (2011) Measurement of ${ }^{13} \mathrm{C}$ and ${ }^{15} \mathrm{~N}$ isotope labeling by gas chromatography/ combustion/ isotope ratio mass spectrometry to study amino fluxes in a plant microbe symbiotic association. Rapid Commun Mass Spectrom 25: 599-607 
Pena R, Offermann C, Simon J, Naumann PS, Gessler A, Holst J, Mayer H, KögelKnabner I, Rennenberg H, Polle A (2010) Girdling affects ectomycorrhizal fungal (EMF) diversity and reveals functional in EMF community composition in a beech forest. Applied and Environmental Studies 76: 1831-1841

Pena R, Simon J, Rennenberg H, Polle A (2012) Ectomycorrhiza affect architecture and nitrogen partitioning of beech (Fagus sylvatica L.) seedlings under shade and drought. Environ Exp Botany 2013: 207 - 217

Peterson BJ \& Fry B (1987) Stable Isotopes in Ecosystem studies. Ann Rev Ecol Sys 18: $293-320$

Read DJ (1992) The mycorrhizal mycelium. In: Allen MF Mycorrhizal functioning. Chapman \& Hall, New York,102-133

Röhrig E, Bartsch N (1992) Waldbau auf ökologischer Grundlage. Erster Band: Der Wald als Vegetationsform und seine Bedeutung für den Menschen. Parey Verlag, Hamburg \& Berlin, 6. Auflage

Schützendübel A, Polle A (2002) Plant responses to abitic stresses: heavy-metal induces oxidative stress and protection by mycorrhization. J Exp Bot 53: 1351-1365

Seven J, Polle A (2014) Subcellular nutrient element localization and enrichment in ecto- and arbuscular mycorrhizas of field-grown beech and ash trees indicate functional differences. PLosOne

Shi L, Guttenberger M, Kottke L, Hampp R (2002) The effect of drought on mycorrhizas on beech (Fagus sylvatica L): changes in community structure and the contentof carbohydrates and nitrogen storage bodies of the fungi. Mycorrhiza 12: 303-311

Simard SW, Perry DA, Jones MD, Myrbold DD, Durall DM, Molina R (1997) Net transfer of carbon between ectomycorrhizal tree species in the field. Nature 388 (6642): 579-582

Smith SE \& Read DJ (1997) Mycorrhizal Symbiosis. Academic press, London

Stinglwagner GKF, Haseder IE, Erlbeck R (2005) Das Kosmos Wald- und Forstlexikon. Kosmos Verlag, Stuttgart, 3. Auflage

Taylor AFS,Martin F, Read DJ (2000) Fungal diversity in ectomycorrhizal communities of Norway spruce (Picea abies L. (Karst)) and beech (Fagus sylvatica) along north-south transects in Europe. Ecological Studies 142:343-365

Tamm CO (1991) Nitrogen in terrestrial ecosystems, questions of productivity, vegetational changes and ecosystem stability. Ecological Studies 81 
2. Kapitel: Stickstoffallokation in den Feinwurzeln der Buche (Fagus sylvatica) in Rein- und Mischbeständen im Nationalpark Hainich

\subsection{Einleitung}

In den Wäldern der gemäßigten und borealen Breiten ist Stickstoff das limitierende Element für das Pflanzenwachstum (Tamm,1991; Vitousek \& Howarth, 1991). Dabei ist Stickstoff ein essentielles Nährelement für Bäume. In Waldbeständen treten die verschiedenen Baumarten zueinander in Konkurrenz, was unter anderem auch bei der Nährstoffakquise (Sadanandan \& Sands, 1993) und der Ausbreitung von Wurzeln im unterirdischen Bereich (Meinen et al., 2009) zum Tragen kommt. Die Buche (Fagus sylvatica) ist in unseren Breitengraden die flächenmäßig dominierende Baumart (Ellenberg \& Leuschner, 2008) und sowohl aus ökologischer als auch ökonomischer Sicht von Bedeutung (Ellenberg \& Strutt, 2009). Sie tritt sowohl in Reinbeständen als auch in Mischbeständen auf und verfügt über eine breite Standortamplitude (Schutt et al., 1992). Zudem wird der Buche ebenfalls eine starke Konkurrenzfähigkeit in Bezug auf die unterirdische Wurzelkonkurrenz attestiert (Rewald \& Leuschner, 2009). Eine besonders hohe Konkurrenzkraft der Buche zeigte sich in Vergesellschaftung mit Fichte (Schmid \& Kazda, 2002; Bolte \& Villaneuva, 2006). Die Produktion von Wurzeln einzelner Baumarten, die zum einen allein, jedoch zum anderen auch in Vergesellschaftung mit anderen Baumarten auftreten, ist Objekt verschiedener Untersuchungsansätze im Freiland (Leuschner et al., 2004; Rewald \& Leuschner, 2009; Meinen et al., 2009; Lei et al., 2012). In diesem Zusammenhang zeigte sich zum Beispiel, dass die Feinwurzelproduktion in Mischbeständen deutlich höher als in Reinbeständen der Buche war (Meinen et al., 2009). Desweiteren zeigte sich, dass die Feinwurzelproduktion der Buche auch bei zunehmender Entfernung zum Stamm stabil hoch bleibt (Lang et al., 2010)

Feinwurzeln spielen auch eine wichtige Rolle für die Aufnahme von Stickstoff in Pflanzen und stellen einen großen Anteil der unterirdischen Biomasse dar (Sanantonio et al., 1977; Persson, 1978). Über das Feinwurzelsystem nehmen Pflanzen Nährstoff auf und transportieren ihn innerhalb der Pflanze (McClaugherty \& Aber, 1982). Eine Untersuchung zur Aufnahmerate von Stickstoff in den Feinwurzeln zeigte zum Beispiel, dass sich Baumarten untereinander unterscheiden (Schulz et al., 2011). Die Aufnahmerate von Stickstoff war dabei Eiche < Buche < Fichte < Kiefer $<$ Linde $<$ Esche. 
Wenn sich Baumarten untereinander in der Aufnahmerate unterscheiden, stellt sich auch die Frage nach ihrem Verhalten in Bezug auf die Stickstoffaufnahme, wenn sie in verschiedener Vergesellschaftung, wie sie in den Wäldern der temperaten Breiten auftreten, vorkommen. In der vorliegenden Untersuchung sollte dazu die Stickstoffakkumulation über einen kurzfristigen Zeitraum in Feinwurzeln der Buche untersucht werden. Der Fokus der Untersuchung lag dabei auf etwaigen Unterschieden in der Akkumulationsdynamik zwischen Rein- und Mischbeständen von Buchenaltbäumen. 
Das Stickstoffmarkierungsexperiment im Freiland wurde vom 21.06.2013 bis 30.06.2013 im Nationalpark Hainich durchgeführt. Die Konstruktionen für die Bodenmarkierung wurden auf den Untersuchungsflächen DL 1a, 1b und 1c sowie DL3a, 3b und 3c des Graduiertenkollegs 1086 installiert. Die Versuchsflächen repräsentierten zwei verschiedene Baumartenzusammensetzungen (Reinbestand und Mischbestand). Die Bezeichnung DL bedeutet Diversitätslevel und beschreibt unter DL 1 Buchenreinbestände und unter DL3 Buchenbestände mit Einmischung von Hainbuche, Linde, Ahorn und Esche.

\subsubsection{Versuchsaufbau}

Die Mittelpunkte der ausgewählten Plots $(50 \mathrm{~m}$ x $50 \mathrm{~m})$ (DI 1a: $51^{\circ} 4^{\prime} 54^{\prime \prime} \mathrm{N}$

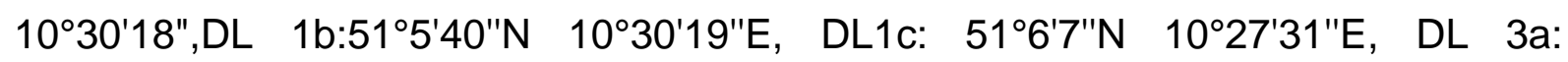

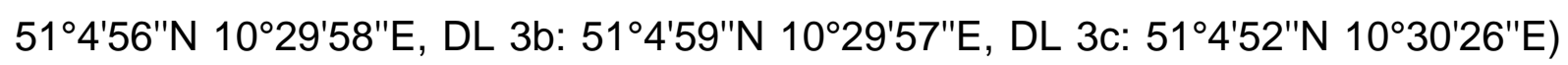
wurden mit einem GPS-Gerät ermittelt ("eTrex 10", Garmin Deutschland GmbH, Garching, Deutschland). Auf jedem Plot wurde unter zwei Buchenaltbäumen die Stickstoffmarkierung durchgeführt und ein weiterer Buchenaltbaum mit Abstand von mindestens $10 \quad \mathrm{~m}$ zu den markierten Bäumen zur Entnahme von Kontrollbodenproben ausgewählt. Daneben wurden auf einigen Plots jeweils ein weiterer Buchenaltbaum für eine Markierung mit farbiger brillant blue-Lösung (15 g auf 1 Liter Wasser) (SERVA Electrophoresis $\mathrm{GmbH}$, Heidelberg, Deutschland) ausgewählt. An Hand der dunkelblauen Lösung wurde der Transport der Markierung im Boden sichtbar gemacht und ein eventuelles "Auslaufen" der Markierungslösung sollte dadurch abgeschätzt werden können (Abbildung 2.1). Um dies zu dokumentieren wurden Bodensäulen mit einem Durchmesser von $5 \mathrm{~cm}$ (innerer Durchmesser $4,7 \mathrm{~cm}$ ) und $20 \mathrm{~cm}$ Tiefe entnommen und der Bohrkern sowie der entstandene Bodenhohlraum betrachtet (Abbildung 2.2.3 und 2.2.4) 


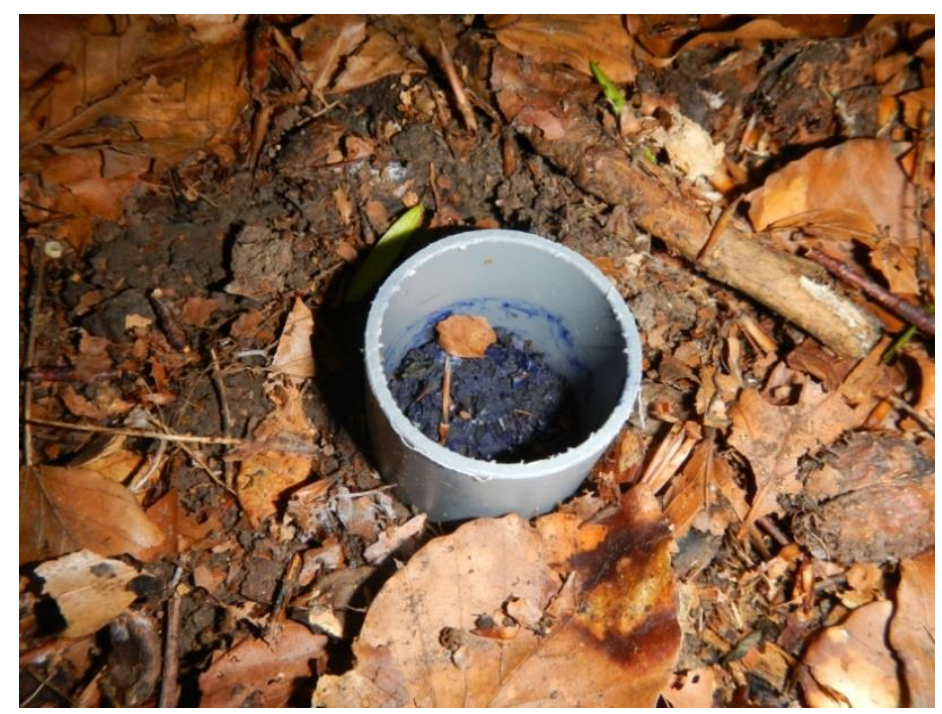

Abbildung 2.2.1: Anfärbung des Bodens mit "brillant blue" (Fotos: J. Sahner)

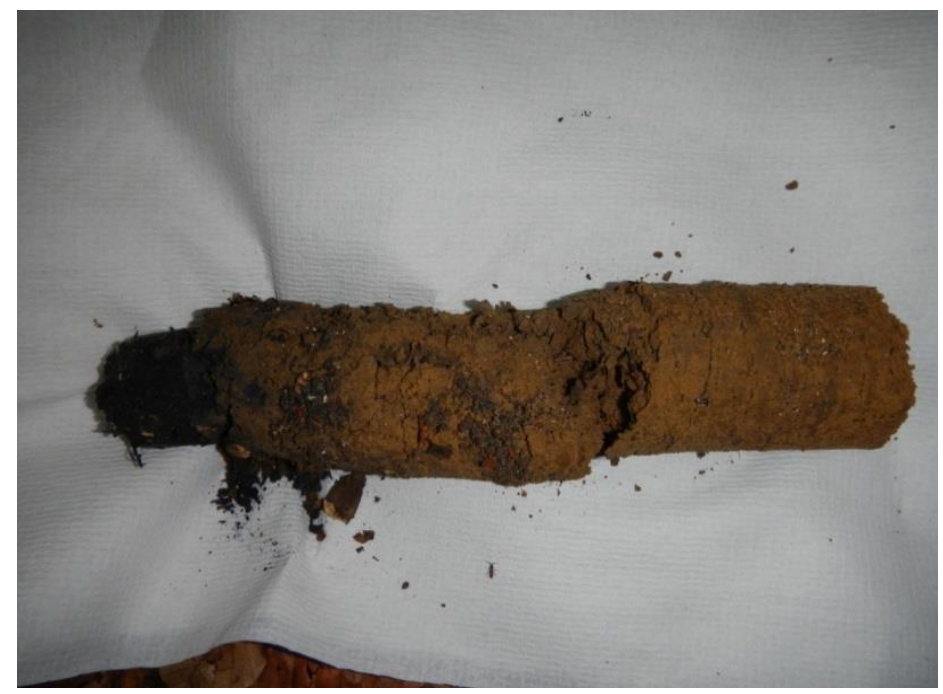

Abbildung 2.2.2: Entnommener Bohrkern nach "brillant blue"- Test 


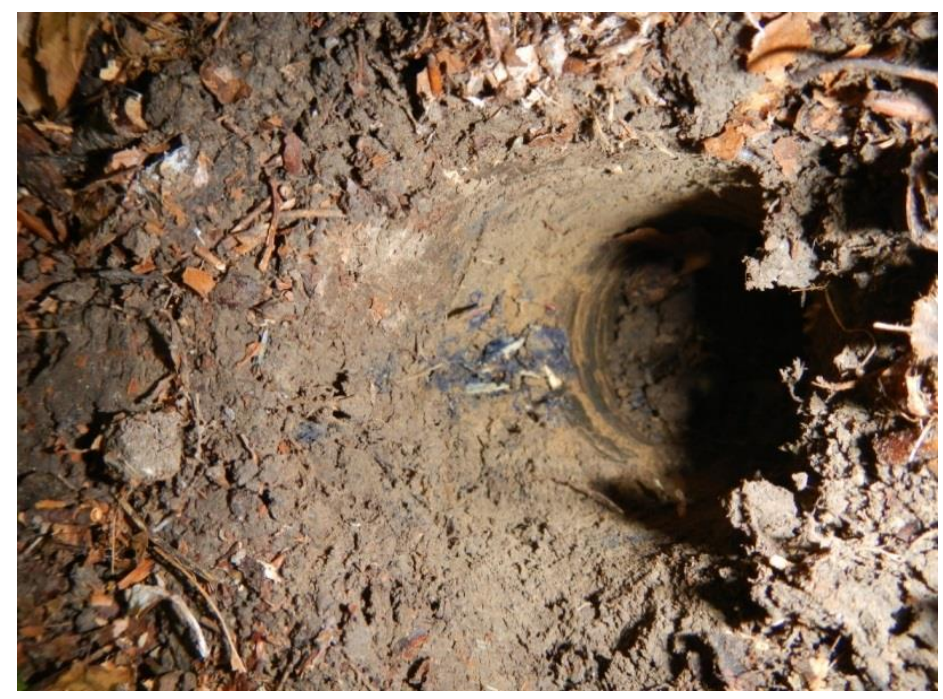

Abbildung 2.2.3: Bodenhohlraum mit ausgelaufener "brillant blue"- Lösung

Zur Stickstoffmarkierung wurde eine 0,05 mol Ammoniumchloridlösung verwendet. Dazu wurden 0,68 g 99\% ${ }^{15} \mathrm{~N}$ - Ammoniumchlorid (Cambridge Isotope Laboratories Inc., Andover, MA, USA) in $250 \mathrm{ml}$ destilliertem Wasser gelöst.

An jedem ausgewählten Baum wurden jeweils 4 Kunststoffringe von $4 \mathrm{~cm}$ Durchmesser im Abstand von einem halben Meter zum Stamm in einer Linie mit jeweils $30 \mathrm{~cm}$ Abstand zueinander in den Boden eingebracht. Die Ringe, in die mit einer Multipipette (Multipipette plus, Eppendorf $\mathrm{GmbH}$, Hamburg, Deutschland) jeweils $10 \mathrm{ml}$ markierte Ammoniumchloridlösung gegeben wurde (Abbildung 2.2.4), wurden zuvor aus Hochtemperatur (HT)-Rohren (Lünemann, Göttingen, Deutschland) auf eine Länge von jeweils $5 \mathrm{~cm}$ geschnitten. 


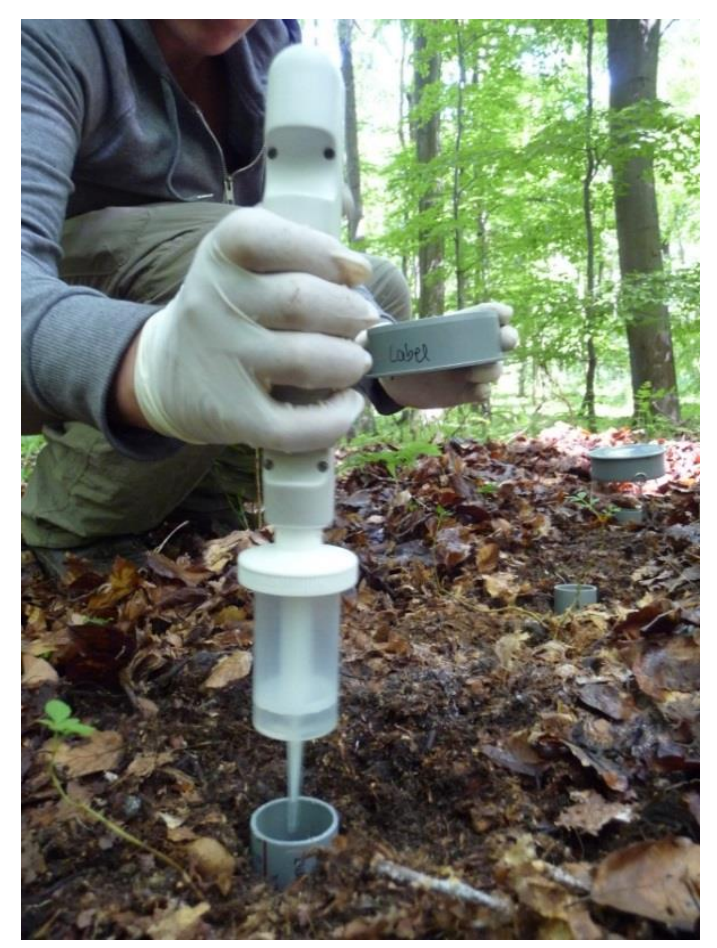

Abbildung 2.2.4: Markierung des Bodens mit ${ }^{15} \mathrm{~N}$-Ammoniumchloridlösung

Die markierten Stellen wurden mit $10 \mathrm{~cm}$-HT-Abschlussdeckeln (Lünemann, Göttingen, Deutschland) in einer Höhe von $15 \mathrm{~cm}$ überdacht (Abbildung 2.2.5 und 2.2.6). Dies sollte das Eindringen von Regen und die Verdünnung der markierten Lösung verhindern. Dazu wurden die Abschlussdeckel an Etikettenspießen mit Doppelöse (Meyer, Rellingen, Deutschland), die auf die entsprechende Länge gekürzt wurden, befestigt und über den Ringen in den Boden eingebracht (Abbildung 2.2.5) . Durch den Abstand der Deckel zum Boden sollte die Luftzirkulation gewährleistet werden und eine eventuelle erhöhte Bakterienaktivität durch verminderte Sauerstoffzufuhr vermieden werden (Abbildung 2.2.6). 


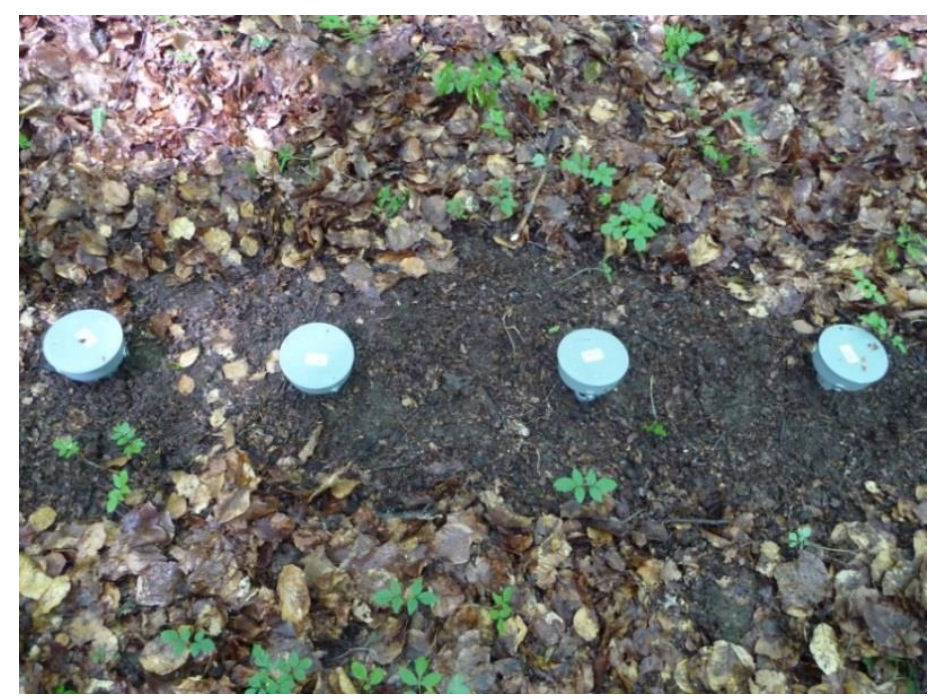

Abbildung 2.2.5: Abdeckung der markierten Stellen zum Schutz vor Regen

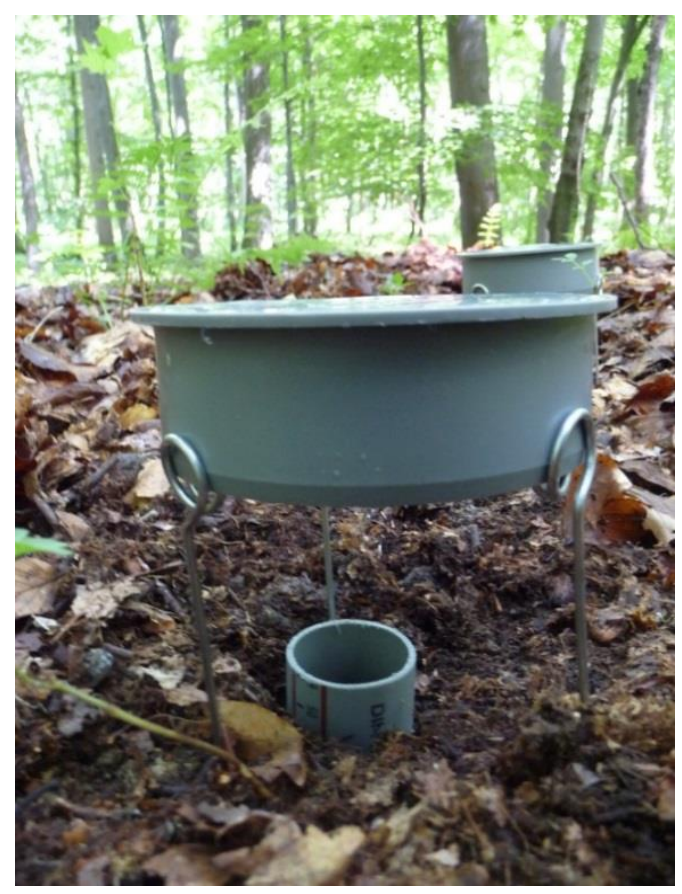

Abbildung 2.2.6: Freiraum zwischen markierter Stelle im Boden und Deckel

Nach 1, 2, 4 und 8 Tagen nach der Markierung wurden Bohrkerne von 4,7 cm Durchmesser (innen) und $20 \mathrm{~cm}$ Länge entnommen. Auf jedem Plot wurde unter beiden markierten Bäumen jeweils ein Bohrkern entnommen und zusätzlich eine Kontrollprobe pro Plot. Daraus ergaben sich über den Experimentverlauf 24 markierte Proben sowie 12 Kontrollproben für den Buchenreinbestand sowie die gleiche Anzahl für den Buchenmischbestand. 


\subsubsection{Probenbearbeitung}

Nach der Entnahme der Bohrkerne wurden diese unverzüglich nach Göttingen gebracht und dort verarbeitet. Aus den Bohrkernen wurden vorsichtig die Wurzeln herausgesucht und mit Hilfe von konventionellen Zahnstochern der Rhizosphärenboden, d.h. der Boden, der direkt der Wurzel anhängt, abgenommen. Der restliche Boden, dessen Volumen durch Entnahme des Rhizosphärenbodens nur gering unter einem Liter lag, wurde mit den Händen gründlich vermischt. Daraus wurde pro Bohrkern eine Bodenmischprobe von rund $30 \mathrm{Gramm}$ Frischgewicht gezogen. Die Feinwurzeln wurden nach Grob- und Feinwurzeln voneinander getrennt und gewogen. Die Grobwurzeln wurden bei $60^{\circ}$ Celsius für eine Woche im Trockenschrank getrocknet (Memmert GmbH \& Co KG, Schwabach, Deutschland).Die Feinwurzeln wurden bis zur weiteren Analyse bei $-20^{\circ}$ Celsius eingefroren. Bodenmischprobe und Rhizosphärenboden wurden gewogen und bei $60^{\circ}$ Celsius für eine Woche im Trockenschrank getrocknet (Memmert GmbH \& Co KG, Schwabach, Deutschland).

\subsubsection{Bestimmung der Isotopensignatur}

Nach dem Trocknen wurden Bodenproben sowie Grobwurzeln gemahlen (MM 2000, Retsch, Haan, Deutschland) und in Zinnkartuschen (4 6 mm, HEKAtech GmbH, Wegberg, Deutschland) eingewogen. Die eingewogene Menge betrug bei den Bodenproben $10 \mathrm{mg}$, bei den Wurzelproben 2-3 mg. Die Isotopenbestimmung erfolgte im KOSI (Kompetenzzentrum Stabile Isotope) des Büsgen-Institutes der Universität Göttingen mit einem Isotopenmassenspektrometer Delta plus (Finnigan MAT, Bremen, Deutschland) und einem Elementaranalysator NA 1500 (FisonsInstruments, Rodano, Milano, Italien) und wurde wie folgend berechnet.

a) Isotopensignatur Kohlenstoff $(\partial \% 0))$

$\partial{ }^{13} \mathrm{C}(\%)=\frac{R \text { Probe }-R \text { Standard }}{R \text { Standard }} * 1000$

mit $R=$ Verhältnis des schweren Isotops $\left({ }^{14} \mathrm{C}\right)$ zu leichtem Isotop $\left({ }^{13} \mathrm{C}\right)$

und Carbonat (Belemnite) aus der PeeDee-Formation (V-PDB) als Standard (Kendall, C., Caldwell, E. A. (1998)) 
b) Isotopensignatur Stickstoff (atom\%)

AP ${ }^{15} \mathrm{~N}($ atom\% $\%)=\frac{15 \mathrm{~N}}{15 \mathrm{~N}+14 \mathrm{~N}} * 100$

mit $\mathrm{R}=$ Verhältnis des schweren Isotops $\left({ }^{15} \mathrm{~N}\right)$ zu leichtem Isotop $\left({ }^{14} \mathrm{~N}\right)$

und Luftstickstoff als Standard

c) Isotopensignatur Stickstoff (Atom\%excess)

APE ${ }^{15} \mathrm{~N}$ (atom\%excess) $=\left(\left(\frac{15 N}{15 N+14 N}\right)\right.$ markierte Probe $-\frac{15 N}{15 N+14 N}$ Kontrolle $) * 100$

2.2.4 Mykorrhizaanalyse

Die eingefrorenen Feinwurzeln wurden zur Charakterisierung der verschiedenen Morphotypen (in Anlehnung an die Methode nach Agerer (1987- 2012)) in Glasschälchen aufgetaut. Da es sich bei den untersuchten Proben um Buchenbestände des Graduiertenkollegs 1086 handelte, die bereits sequenziert wurden, wurde der zugehörige institutseigene Katalog goe-fungi (Christa Lang, 2008, http://www.uni-goettingen.de/de/92389.html) zur Ansprache der Morphotypen hinzugezogen. In der ersten Phase des Graduiertenkollegs wurde dieser Katalog auf Grundlage der untersuchten Flächen angefertigt. Der Katalog beschreibt die Ektomykorrhizaarten des Hainichs mit entsprechenden Fotos und enthält Sequenzierungsdaten der Pilzarten der verschiedenen Laubbaumarten des Hainichs. Unter einer Stereolupe (M 205 FA, Leica, Wetzlar, Deutschland) wurden die Feinwurzeln mit ihren assoziierten Morphotypen betrachtet. An Hand ihrer Farbe, Verzweigung, dem Auftreten von Hyphen und der Struktur der Manteloberfläche wurden die pilzlichen Strukturen in Morphotypen eingeteilt. In jeder Probe (im Sinne von geerntetem soil core) wurden bis zu 300 Wurzelspitzen ausgezählt. Es traten in den untersuchten Proben auch soil cores auf, die lediglich 100 Wurzelspitzen insgesamt aufwiesen. Die Wurzelspitzen eines jeden soil cores setzten sich aus vitalen und trockenen Wurzelspitzen zusammen. Es sollten insgesamt 1000 vitale Wurzelspitzen pro Diversitätslevel gezählt werden. Dies diente zur Untersuchung, ob es einen Unterschied in der Mykorrhizagesellschaft zwischen den beiden Diversitätsleveln gibt. $\mathrm{Da}$ davon ausgegangen wurde, dass sich die Zusammensetzung der Mykorrhizagesellschaft nicht innerhalb des kurzen Versuchszeitraums von acht Tagen veränderte, wurden von jedem Versuchstag 2-3 
soil cores pro Diversitätslevel ausgezählt und addiert, um insgesamt eine Anzahl von 1000 vitalen Wurzelspitzen pro Diversitätslevel zu erreichen.

\subsubsection{Mykorrhizierungs- und Vitalitätsrate}

Auf Grundlage der gezählten Wurzelspitzen wurden die Mykorrhizierungsrate der

Buchenwurzeln (a) sowie die Vitalitätsrate der gezählten Wurzelspitzen (b)

untersucht. Bei den Zählungen der Wurzelspitzen wurden die einzelnen

Morphotypen, wie oben beschrieben, charakterisiert. Bei den trockenen

Wurzelspitzen wurde jedoch nicht nach "mykorrhiziert" und "nicht-mykorrhiziert" unterschieden.

a) Mykorrhizierungsrate (\%)

$=\frac{\text { Anzahl mykorrhizierter WS }}{\text { Anzahl nicht-mykorrhizierter WS + mykorrhizierter WS }}{ }^{*} 100$

b) Vitalitätsrate (\%)

$=\quad \frac{\text { Anzahl nicht-mykorrhizierter WS + mykorrhizierter WS }}{\text { Anzahl aller gezählten WS }} * 100$

\subsubsection{Diversitätsindex Shannon-Index}

Die Diversität der Pilzarten in den verschiedenen Diversitätsleveln der

Buchenbestände wurde mit Hilfe der Software EstimateS 8.2 (RK Coldwell, Storrs, USA) berechnet. Diesem Index liegt folgende Berechnung zu Grunde:

$\Sigma^{S} p_{i}{ }^{*} \ln p_{i} \quad$ mit $p_{i}=n_{i} / N$ und $\sum p_{i}=1$

Dabei ist $p_{i}$ die relative Häufigkeit der i-ten Art von der Gesamtanzahl von Individuen $(\mathrm{N})$ mit $\mathrm{n}_{\mathrm{i}}$ als Individuenanzahl der Art i und einer Gesamtartenanzahl $\mathrm{S}$. Der Shannon-Index wurde ebenfalls in Bezug auf die Anzahl an Baumarten in beiden Diversitätslevel berechnet.

\subsubsection{Statistik}

Die statistische Analyse erfolgte mit Microsoft Excel (Windows, Redmond, USA) und OriginPro 8.5 (OriginLab Corporation, Northampton,USA). Die Daten wurden an Hand von Shapiro-Wilk- bzw. Kolmogorov-Smirnov-Test auf Normalverteilung gestest. Normalverteilte Daten wurden an Hand von ANOVA analysiert. Zur Analyse von nicht-normalverteilten Daten wurde Kruskal-Wallis-ANOVA als nichtparametrischer Test verwendet. 


\subsection{Ergebnisse}

\subsubsection{Biomasse}

Das Frischgewicht der entnommenen Bodenkerne betrug im Buchenreinbestand (DL1) zwischen 246, $04 \pm$ 18,3 $\mathrm{g}$ und 276,50 \pm 21,6 $\mathrm{g}$ und zwischen 243,09 \pm 17,1 $\mathrm{g}$ und 268,76 $\pm 8,7 \mathrm{~g}$ in den Buchenmischbeständen (DL3) (Tabelle 2.3.1). Das Frischgewicht der aus den beiden unterschiedlichen Diversitätsleveln entnommenen Bodenkernen unterschied sich nicht signifikant zwischen Rein- und Mischbestand (Tabelle 2.3.1). Die Frischgewichte der Feinwurzeln in DL1 betrugen zwischen 0,63 \pm 0,1 $\mathrm{g}$ und 1,48 \pm 0,3 $\mathrm{g}$ und in DL 3 zwischen 0,99 $\pm 0,2 \mathrm{~g}$ und 2,07 $\pm 0,2 \mathrm{~g}$ (Tabelle 2.3.1). Es handelte sich dabei um alle aus dem Bodenkern aussortierten Feinwurzeln. In DL 1 handelte es sich dabei ausschließlich um Buchenwurzeln und in DL 3 um die Summe aller enthaltenen Baumarten. Die reine Buchenwurzelmasse in DL3 wurde im Frischgewicht nicht gesondert bestimmt. Die Feinwurzelmenge unterschied sich zwischen Rein- und Mischbestand signifikant (Tabelle 2.3.1). Das Frischgewicht der Grobwurzeln lag in DL 1 zwischen 1,37 \pm 0,7 g und 3,08 \pm 1,6 g sowie zwischen 1,55 0,7 und 6,08 \pm 3,8 g in DL 3 (Tabelle 2.3.1.) In Bezug auf die Grobwurzelmenge wurde kein signifikanter Unterschied zwischen den beiden Diversitätsleveln festgestellt (Kruskal-Wallis-ANOVA für nicht-normalverteilte Daten;

Tabelle 2.3.1)

Tabelle 2.3.1: Frischgewichte $(\mathrm{g})$ von entnommenem Boden und darin befindlichen Fein- und Grobwurzeln in den Buchenreinbeständen (DL1) und Buchenmischbeständen(DL3) an den Versuchstagen 1,2,4, und 8 sowie Tag 0 als Kontrolle. $\mathrm{n}=$ Anzahl an Wiederholungen pro Tag und Diversitätslevel (DL1, DL3). Daten zeigen Mittelwerte (MW) und Standardfehler (SE).

\begin{tabular}{|c|c|c|c|c|c|c|c|}
\hline \multirow[b]{2}{*}{ Tag } & \multirow[b]{2}{*}{ Kompartiment } & \multirow[b]{2}{*}{$\begin{array}{c}\text { Bodentiefe } \\
\mathrm{cm}\end{array}$} & \multirow[b]{2}{*}{$\mathbf{n}$} & \multicolumn{4}{|c|}{ Diversitätslevel } \\
\hline & & & & $\begin{array}{c}\text { DL1 } \\
\text { FG (g) } \\
M W \pm S E\end{array}$ & $\begin{array}{c}\text { DL3 } \\
\text { FG (g) } \\
M W \pm S E\end{array}$ & $\begin{array}{c}\mathbf{p} \\
\text { (Treatment) }\end{array}$ & $\begin{array}{c}p \\
(D L)\end{array}$ \\
\hline 0 & Boden & $0-10$ & 12 & $247,34 \pm 8,0$ & $268,76 \pm 8,7$ & & \\
\hline 1 & Boden & $0-10$ & 6 & $276,50 \pm 21,6$ & $254,43 \pm 13,6$ & & \\
\hline 2 & Boden & $0-10$ & 6 & $270,86 \pm 8,8$ & $259,20 \pm 6,1$ & 0,99 & 0,68 \\
\hline 4 & Boden & $0-10$ & 6 & $265,79 \pm 12,3$ & $243,09 \pm 17,1$ & & \\
\hline 8 & Boden & $0-10$ & 6 & $246,05 \pm 18,3$ & $249,17 \pm 10,9$ & & \\
\hline 0 & Feinwurzel & $0-10$ & 12 & $1,01 \pm 0,1$ & $2,07 \pm 0,2$ & & \\
\hline 1 & Feinwurzel & $0-10$ & 6 & $1,48 \pm 0,3$ & $2,05 \pm 0,5$ & & \\
\hline 2 & Feinwurzel & $0-10$ & 6 & $1,39 \pm 0,2$ & $1,91 \pm 0,2$ & 0,032 & $<0,01$ \\
\hline 4 & Feinwurzel & $0-10$ & 6 & $0,98 \pm 0,2$ & $1,41 \pm 0,3$ & & \\
\hline 8 & Feinwurzel & $0-10$ & 6 & $0,63 \pm 0,1$ & $0,99 \pm 0,2$ & & \\
\hline 0 & Grobwurzel & $0-10$ & 12 & $2,06 \pm 1,2$ & $2,06 \pm 1,2$ & & \\
\hline 1 & Grobwurzel & $0-10$ & 6 & $2,00 \pm 1,1$ & $6,08 \pm 3,8$ & & \\
\hline 2 & Grobwurzel & $0-10$ & 6 & $1,37 \pm 0,7$ & $1,55 \pm 0,7$ & 0,62 & 0,13 \\
\hline 4 & Grobwurzel & $0-10$ & 6 & $1,56 \pm 0,5$ & $2,85 \pm 0,8$ & & \\
\hline 8 & Grobwurzel & $0-10$ & 6 & $3,08 \pm 1,6$ & $1,69 \pm 0,4$ & & \\
\hline
\end{tabular}


Das Trockengewicht der Feinwurzeln betrug in DL1 zwischen $0,20 \pm 0,1 \mathrm{~g}$ und $0,49 \pm$ $0,1 \mathrm{~g}$ sowie zwischen $0,12 \pm 0,04 \mathrm{~g}$ und $0,85 \pm 0,2 \mathrm{~g}$ in DL3 (Tabelle 2.3.2). Dabei handelte es sich ebenfalls um alle, im Bodenkern befindlichen Feinwurzeln. Im Trockengewicht wurde bei den Feinwurzeln kein signifikanter Unterschied festgestellt (Tabelle 2.3.2). Das Trockengewicht der Grobwurzeln betrug in den Buchenreinbeständen zwischen $0,82 \pm 0,3$ und $1,78 \pm 0,6$ und in den Buchenmischbeständen zwischen $0,80 \pm 0,3$ und $3,41 \pm 2,1 \mathrm{~g}$ (Tabelle 2.3.2).

Tabelle 2.3.2: Trockengewichte (g) von Fein- und Grobwurzeln in den Bodenkernen in den Buchenreinbeständen (DL1) und Buchenmischbeständen(DL3) an den Versuchstagen 1,2,4, und 8 sowie Tag 0 als Kontrolle. $\mathrm{N}=$ Anzahl an Wiederholungen pro Tag und Diversitätslevel (DL1, DL3). Daten zeigen Mittelwerte (MW) und Standardfehler (SE). $\mathrm{P}=$ statistische Signifikanz

\begin{tabular}{|c|c|c|c|c|c|c|c|}
\hline \multirow[b]{2}{*}{ Tag } & \multirow[b]{2}{*}{ Kompartiment } & \multirow[b]{2}{*}{$\begin{array}{c}\text { Bodentiefe } \\
\mathrm{cm}\end{array}$} & \multirow[b]{2}{*}{$\mathbf{n}$} & \multicolumn{2}{|c|}{ Diversitätslevel } & \multirow[b]{2}{*}{$\begin{array}{c}\mathbf{p} \\
\text { (Treatment) }\end{array}$} & \multirow[b]{2}{*}{$\begin{array}{c}p \\
(D L)\end{array}$} \\
\hline & & & & $\begin{array}{c}\text { DL1 } \\
\text { FG (g) } \\
M W \pm S E\end{array}$ & $\begin{array}{c}\text { DL3 } \\
\text { FG (g) } \\
M W \pm S E\end{array}$ & & \\
\hline 0 & Feinwurzel & $0-10 \mathrm{~cm}$ & 5 & $0,27 \pm 0,3$ & $0,17 \pm 0,1$ & \multirow{5}{*}{0,224} & \multirow{5}{*}{0,810} \\
\hline 1 & Feinwurzel & $0-10 \mathrm{~cm}$ & 3 & $0,36 \pm 0,1$ & $0,85 \pm 0,2$ & & \\
\hline 2 & Feinwurzel & $0-10 \mathrm{~cm}$ & 4 & $0,49 \pm 0,1$ & $0,17 \pm 0,0$ & & \\
\hline 4 & Feinwurzel & $0-10 \mathrm{~cm}$ & 5 & $0,34 \pm 0,1$ & $0,12 \pm 0,0$ & & \\
\hline 8 & Feinwurzel & $0-10 \mathrm{~cm}$ & 5 & $0,20 \pm 0,1$ & $0,37 \pm 0,1$ & & \\
\hline 0 & Grobwurzel & $0-10 \mathrm{~cm}$ & 12 & $1,02 \pm 1,5$ & $1,28 \pm 1,6$ & \multirow{5}{*}{0,788} & \multirow{5}{*}{0,373} \\
\hline 1 & Grobwurzel & $0-10 \mathrm{~cm}$ & 6 & $1,78 \pm 0,6$ & $3,41 \pm 2,1$ & & \\
\hline 2 & Grobwurzel & $0-10 \mathrm{~cm}$ & 6 & $0,83 \pm 0,4$ & $0,80 \pm 0,3$ & & \\
\hline 4 & Grobwurzel & $0-10 \mathrm{~cm}$ & 6 & $0,82 \pm 0,3$ & $1,54 \pm 0,5$ & & \\
\hline 8 & Grobwurzel & $0-10 \mathrm{~cm}$ & 6 & $1,56 \pm 0,9$ & $0,91 \pm 0,2$ & & \\
\hline
\end{tabular}

Die Berechnung des Shannon-Index in Bezug auf die Baumartenanzahl in den beiden Diversitätsleveln zeigte einen signifikanten Unterschied zwischen beiden. Der Shannon-Index in DL 1 betrug 1 und in DL 3 2,15 (Tabelle 2.3.3).

In der Analyse wurden ebenfalls die Mykorrhizierungs- und Vitalitätsrate der Wurzeln sowie der Shannon-Index der Pilzarten bestimmt (Tabelle 2.3.3). Die Vitalitätsrate war mit 56,4 $\pm 5,3 \%$ in den Buchereinbeständen deutlich höher als in den Buchenmischbeständen, in denen eine durchschnittliche Vitalitätsrate von 35, $1 \pm 3,5$ $\%$ bestimmt wurde (Tabelle 2.3.3). Dies zeigte einen signifikanten Unterschied zwischen Rein- und Mischbestand (Tabelle 2.3.3) Die Mykorrhizierungsrate unterschied sich zwischen Rein- und Mischbeständen jedoch nicht und betrug in den Altbeständen $100 \pm 0 \%$ der vitalen Wurzelspitzen (Tabelle 2.3.3). Durch die 
identische Mykorrhizierungsrate in den Beständen konnte kein statistischer Test verwendet werden (Tabelle 2.3.3). Der berechnete Shannon-Index betrug im Buchenreinbestand 2,22 und im Buchenmischbestand 2,09 (Tabelle 2.3.3).

Tabelle 2.3.3: Shannon-Index von Wurzeln und Mykorrhizapilzen in DL1 und DL3 sowie Vitalitäts- und Mykorrhizierungsrate. Daten zeigen $\mathrm{MW} \pm \mathrm{SE} ; \mathrm{p}=$ statistische Signifikanz

\begin{tabular}{|c|c|c|c|}
\hline \multirow{2}{*}{ Parameter } & \multicolumn{2}{|c|}{ Diversitätslevel } & \\
& $\begin{array}{c}\text { DL1 } \\
\text { MW } \pm \text { SE }\end{array}$ & $\begin{array}{c}\text { DL3 } \\
\text { MW } \pm \text { SE }\end{array}$ & p \\
\hline Shannon-Index (Wurzeln) & 1 & 2,15 & $<0,05$ \\
\hline Vitalitätsrate (Wurzeln) in \% & $56,4 \pm 5,2$ & $35,1 \pm 3,5$ & $<0,01$ \\
\hline Mykorrhizierungsrate (Wurzeln) & $100 \pm 0$ & $100 \pm 0$ & n.s. \\
\hline Shannon-Index (Mykorrhiza) & 2,22 & 2,06 & n.s. \\
\hline
\end{tabular}

\section{3. $2{ }^{15} \mathrm{~N}$-Allokation in den Wurzeln und im Boden}

Um zu prüfen, ob die N-Aufnahme in DL1 und DL3 in Buchenwurzeln ähnlich verlief, wurde die Akkumulation von ${ }^{15} \mathrm{~N}$ über die natürliche Signatur von ${ }^{15} \mathrm{~N}$ hinaus bestimmt. Dies wurde im Folgenden als APE (atom\%excess) abgekürzt. Tabelle 2.3.4 zeigt die atom\% ${ }^{15} \mathrm{~N}$-Werte sowie APE der Kontrollen von Feinwurzeln, Grobwurzeln, Rhizosphärenboden und Boden.

Tabelle 2.3.4: Atom\%- ${ }^{-15} \mathrm{~N}$ in den Kontrollen von Feinwurzeln (FW), Grobwurzeln (GW), Rhizosphärenboden (RB) und Boden (BM) im Rein- (DL1) und Mischbestand (DL3); Tag $0=$ Kontrolle; Daten zeigen MW \pm SE, Ein-Weg-ANOVA zwischen DL1 und DL3;

\begin{tabular}{|c|c|c|c|c|c|c|c|c|c|}
\hline TAG & DL & KOMP. & $\mathbf{n}$ & $\begin{array}{c}\mathbf{1 5 N} \\
\text { ATOM \% }\end{array}$ & & $\mathbf{p}$ & $\begin{array}{c}\mathbf{1 5 N} \\
\text { APE\% }\end{array}$ & & $\mathbf{p}$ \\
& & & & $\mathbf{M W}$ & $\mathbf{S E}$ & & $\mathbf{M W}$ & $\mathbf{S E}$ & \\
\hline 0 & 1 & FW & 5 & 0,36601 & 0,00015 & 0,593 & $-2,22 \mathrm{E}-17$ & 0,00015 & 1 \\
0 & 3 & FW & 5 & 0,36614 & 0,00017 & & $1,11 \mathrm{E}-17$ & 0,00017 & \\
\hline 0 & 1 & GW & 7 & 0,36459 & 0,00014 & 0,040 & $1,59 \mathrm{E}-17$ & 0,00014 & 1 \\
0 & 3 & GW & 5 & 0,36419 & 0,00007 & & $-1,11 \mathrm{E}-17$ & 0,00007 & \\
\hline 0 & 1 & RB & 4 & 0,36483 & 0,00012 & 0,672 & $-1,39 \mathrm{E}-17$ & 0,00012 & 1 \\
0 & 3 & RB & 5 & 0,36488 & 0,00002 & & $6,66 \mathrm{E}-17$ & 0,00002 & \\
\hline 0 & 1 & BM & 5 & 0,36492 & 0,00008 & 0,786 & $-1,39 \mathrm{E}-17$ & 0,00008 & 1 \\
0 & 3 & BM & 5 & 0,36489 & 0,00009 & & $3,33 \mathrm{E}-17$ & 0,00009 & \\
\hline
\end{tabular}

Sowohl im Rein- als im Mischbestand war zu beobachten, dass APE über den Versuchszeitraum von 8 Tagen im Vergleich zu den Kontrollen (Tabelle 2.3.4) anstieg (Abbildung 2.3.2 a), wobei die Akkumulation in den Feinwurzeln im 
Reinbestand stets höher war als im Mischbestand. Es zeigte sich ein signifikanter Unterschied (Tabelle 2.3.5) zwischen den beiden Beständen.

Es war zu beobachten, dass die Akkumulation von ${ }^{15} \mathrm{~N}$ in allen Kompartimenten, besonders jedoch in den Feinwurzeln, in den ersten Tagen nach der Markierung erfolgte (Abbildung 2.3.2). Auch bei den Grobwurzeln eine signifikante Anreicherung von APE in den markierten Proben im Vergleich zu den Kontrollen beobachtet werden (Tabelle 2.3.5) (Abbildung 2.3.2 b). Bei den beobachteten tendenziell unterschiedlichen Verläufen in Rein- und Mischbestand konnte bei der statistischen Analyse jedoch kein signifikanter Unterschied nachgewiesen werden (Tabelle 2.3.5), was auch in Bezug auf Unterschiede an den einzelnen Tagen überprüft wurde ( $p$ Tag1 $=1, p_{\text {Tag } 2}=0,08, p_{\text {Tag }} 4=0,646, p_{\text {Tag }} 8=0,932$ ).

Im Rhizosphärenboden zeigte sich in beiden Beständen eine signifikante Erhöhung von APE ${ }^{15} \mathrm{~N}$ nach der Markierung (Tabelle 2.3.5). Über den Versuchszeitraum von 8 Tagen stieg die Akkumulation von APE im Reinbestand bis Tag 2 und verblieb dann auf einem konstanten Niveau (Abbildung 2.3.2 c). Im Vergleich dazu stieg die Akkumulation im Mischbestand von einem niedrigeren Niveau bis Tag 4, danach höher als im Reinbestand (Abbildung 2.3.2 c). Es konnte jedoch kein signifikanter Unterschied in der Akkumulation von APE im Rhizosphärenboden von Rein- und Mischbestand belegt werden (Tabelle 2.3.4). Die Untersuchung der beiden Diversitätslevel an einzelnen Messtagen ergab, dass lediglich am letzten Versuchstag ein signifikanter Unterschied in der Akkumulation im Rhizosphärenboden auftrat ( $\mathrm{p}_{\text {Tag }}=4,72 \mathrm{e}^{-4}$, Ein-Weg-ANOVA). Im Boden (bezeichnet als „bulk soil“) konnte beobachtet werden, dass sich die Akkumulation über der Kontrolle zum ersten Versuchstag erhöhte (Tabelle 2.3.5) und dann bis zum Versuchsverlauf in beiden Beständen auf einem konstanten Niveau verblieb (Abbildung 2.3.2 d). Zwischen den beiden Beständen wurde im markierten Boden jedoch kein signifikanter Unterschied beobachtet (Tabelle 2.3.5). 


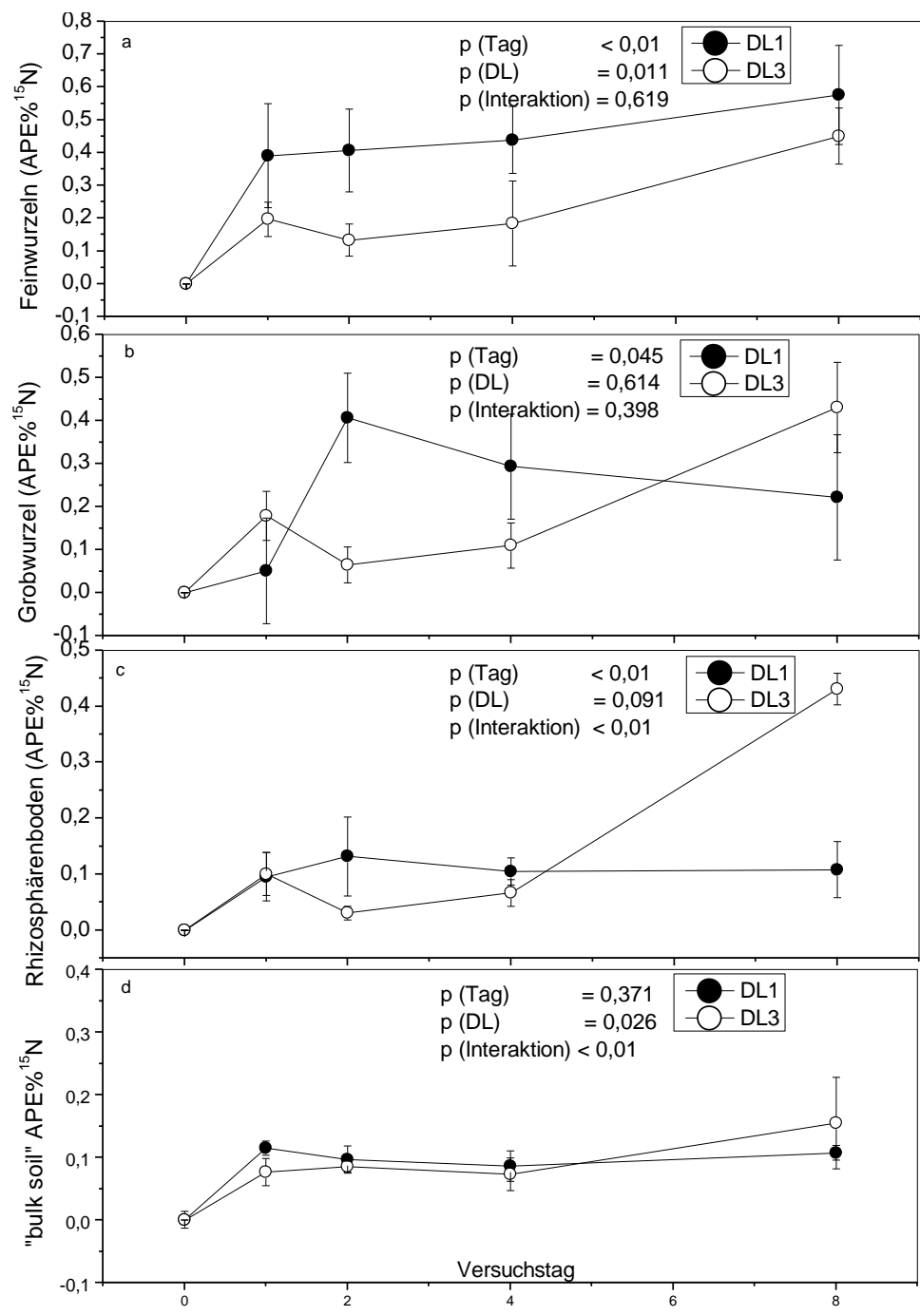

Abbildung 2.3.2: APE ${ }^{15} \mathrm{~N}$ - Akkumulation in Feinwurzeln (a), Grobwurzeln (b), Rhizosphärenboden (c) und „bulk soil“(d) in Rein- (DL1) und Mischbestand (DL 3) über einen Versuchszeitraum von 8 Tagen in Kontrollen (Tag 0) und markierten Proben (Tag 1,2,4,8). DL= Diversitätsleveln; Daten zeigen MW \pm SE. $n=3-6$ (Feinwurzeln), $n=5-7$ (Grobwurzeln), $n=3-6$ (Rhizosphärenboden), $n=4-5$ (,bulk soil“) pro Tag und Treatment. Treatment: Kontrolle bzw. markiert

Tabelle 2.3.5: $p$-Werte der Zwei-Wege- ANOVA von APE ${ }^{15} \mathrm{~N}$ in Feinwurzeln, Grobwurzeln, Rhizosphärenboden und Mischboden. Faktoren: DL (Reinbestand bzw. Mischbestand); Tag: 0 als Kontrolle und 1,2,4 bzw. 8 als markierte Probe.

\begin{tabular}{|c|c|c|c|}
\hline Kompartiment & $\mathbf{P}$ (Tag) & $\mathbf{P}$ (DL) & $\mathbf{P}$ (Interaktion) \\
\hline Feinwurzeln & $<0,01$ & 0,011 & 0,619 \\
\hline Grobwurzeln & 0,045 & 0,614 & 0,398 \\
\hline Rhizosphärenboden & $<0,01$ & 0,091 & $<0,01$ \\
\hline Boden & $<0,01$ & 0,870 & 0,696 \\
\hline
\end{tabular}




\subsubsection{Charakterisierung der Mykorrhizagesellschaft}

In einer zufälligen Auswahl der Feinwurzelproben wurde die Mykorrhizagesellschaft in Rein- und Mischbestand im oberen Bodenbereich (0-10 cm Tiefe) betrachtet; dabei wurde davon ausgegangen, dass die Mykorrhizagesellschaft in dem kurzen Versuchszeitraum keiner Veränderung unterlag. Zudem wurde ein Einfluss der Stickstoffmarkierung auf die Zusammensetzung der Pilzarten innerhalb der Gesellschaft ebenfalls ausgeschlossen. Daher wurde die Charakterisierung im Hinblick auf Unterschied zwischen Rein- und Mischbestand der Buche durchgeführt. In beiden Beständen wurden folgende Pilzarten beobachtet: Clavulina cristata, Tuber puberulum, Humaria hemisphaerica, Tomentella sublilacina, Lactarius subdulcis, Lactarius blennius, Cenococcum geophilum, Genea hispidula, Russula fellea und Xerocomus chrysenteron. Die Gesamtanzahl der beobachteten Individuen betrugen in DL1 1013 Individuen und in DL 31014 Individuen (Tabelle 2.3.6) Die Anzahl der beobachteten Individuen in Bezug auf das Diversitätslevel sind in Tabelle 2.3.8 aufgeführt. Daneben wurden im Reinbestand Individuen von Inocybe glabris, die im Mischbestand nicht auftraten sowie von Laccaria spec., von dem im Mischbestand lediglich 2 Individuen beobachtet wurden (Tabelle 2.3.6). Es zeigte sich, dass die Pilzarten Clavulina cristata, Lactarius subdulcis, und Russula fellea in beiden Diversitätsleveln am häufigsten auftraten (Abbildung 2.3.3). Es zeigte sich, dass die auftretenden Pilzarten in beiden Diversitätsleveln annähernd gleich waren, sich jedoch in ihrer Abundanz signifikant zwischen DL1 und DL3 unterschieden (ANOSIM, $p=0,0002$ ). Die unterschiedliche Häufigkeit der beobachteten Pilzarten ist Tabelle 2.3.6 zu entnehmen. 
Tabelle 2.3.6: Anzahl der beobachteten Individuen in der Bodentiefe $0-10 \mathrm{~cm}$ in Rein- und Mischbestand. Daten zeigen Summen über alle gezählte soil cores. Anzahl der soil cores beträgt im Reinbestand 6 sowie im Mischbestand 11.

\begin{tabular}{|c|c|c|}
\hline Pilzart & $\begin{array}{l}\text { Summe der Individuen } \\
\text { im Reinbestand (DL1) }\end{array}$ & $\begin{array}{l}\text { Summe der Individuen } \\
\text { im Mischbestand (DL3) }\end{array}$ \\
\hline Clavulina cristata & 237 & 146 \\
\hline Tuber puberulum & 77 & 155 \\
\hline Humaria hemisphaerica & 110 & 36 \\
\hline Tomentella sublilacina & 58 & 12 \\
\hline Lactarius subdulcis & 147 & 183 \\
\hline Cenococcum geophilum & 48 & 113 \\
\hline Lactarius blennius & 14 & 159 \\
\hline Genea hispidula & 30 & 60 \\
\hline Russula fellea & 146 & 131 \\
\hline Inocybe glabris & 48 & 0 \\
\hline Laccaria spec. & 15 & 2 \\
\hline $\begin{array}{l}\text { Xerocomus } \\
\text { chrysenteron }\end{array}$ & 83 & 17 \\
\hline Summe & 1013 & 1014 \\
\hline
\end{tabular}




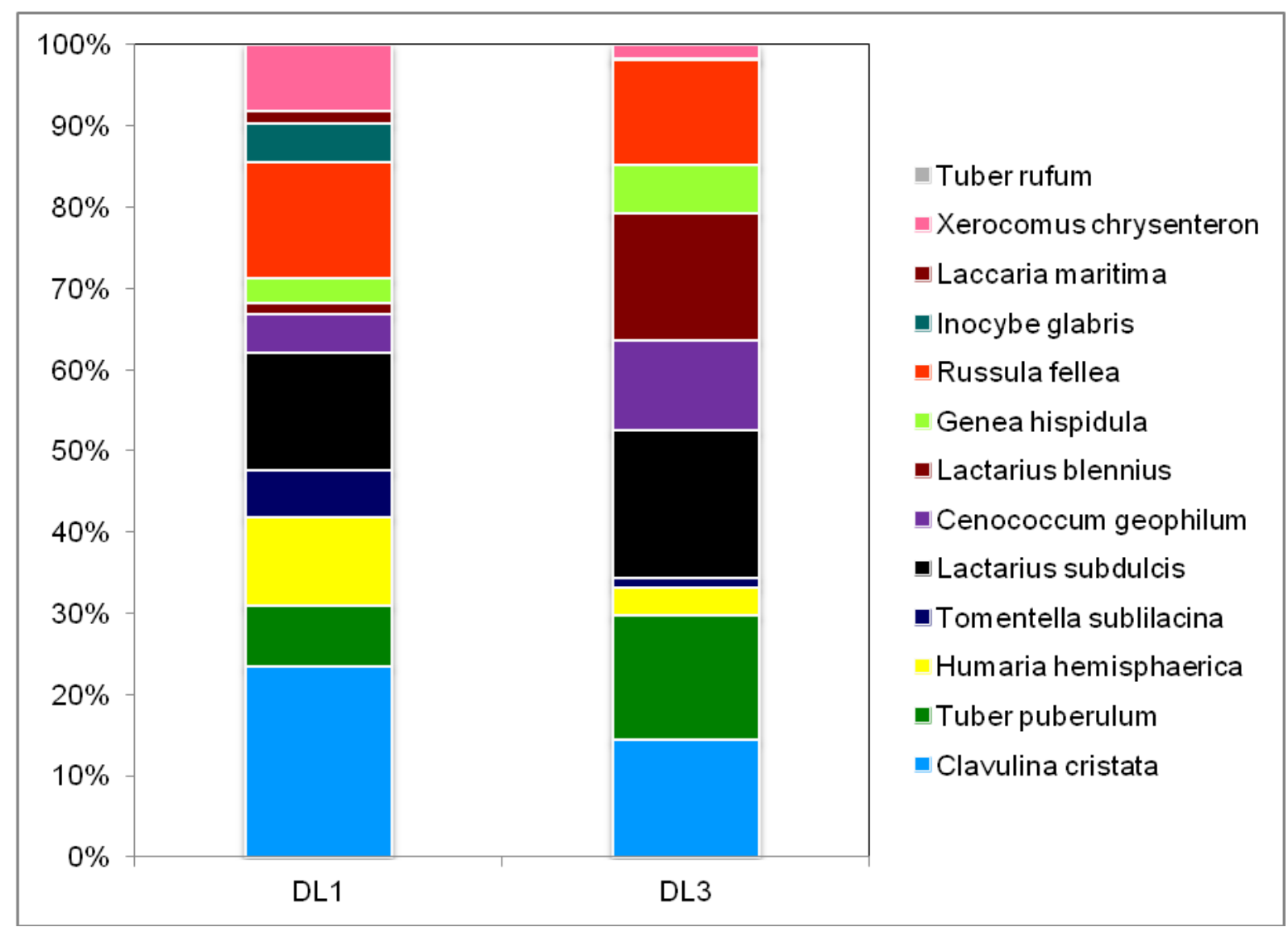

Abbildung 2.3.3: Relative Abundanzen der beobachteten Pilzarten in Reinbestand (DL1) und Mischbestand (DL3) 


\subsection{Diskussion}

In der vorliegenden Untersuchung wurde die kurzfristige Stickstoffakkumulation in Feinwurzeln in Buchenreinbeständen sowie Beständen der Buche unter Einmischung verschiedener Laubbaumarten im Nationalpark Hainich in Thüringen bestimmt.

Ausgehend von der Probenentnahme aus dem Freilandboden zeigte sich kein Unterschied in der frischen Bodenmenge, was auf die methodisch gleiche Vorgehensweise sowie der gleichen Dichte und des gleiche Wassergehaltes in beiden Beständen zurückzuführen ist. Die Analyse der aus den Bodenkernen separierten Feinwurzeln zeigte, dass die Feinwurzelmenge im Frischzustand im Mischbestand höher als im Reinbestand war. Dies ist vermutlich darin begründet, dass der Mischbestand ebenfalls Wurzeln anderer Baumarten enthielt, wobei der Einfluss des enthaltenen Wassers nicht ausgeschlossen werden darf. Weitergehend zeigte sich nämlich bei der Analyse der Trockenmasse der Feinwurzeln, kein signifikanter Unterschied in der Feinwurzelmasse zwischen Rein- und Mischbestand der Buche. Dieses Ergebnis geht konform mit Beobachtungen von Meinen et al. (2009 a), die jedoch in anderen Untersuchungen, ebenfalls auf den gleichen Versuchsflächen wie in der vorliegenden Untersuchung, eine tendenziell, jedoch nicht signifikant, höhere Feinwurzelmasse $\left(\mathrm{g} / \mathrm{cm}^{2}\right)$ in Mischbeständen beobachtete (Meinen et al., 2009 b). Die Mykorrhiza der Buchenfeinwurzeln wurde in beiden Beständen auf ihre Mykorrhizierungs- und Vitalitätsrate hin untersucht. Die Mykorrhizierungsrate war dabei in Rein- und Mischbestand sehr hoch, wie es der generellen Mykorrhizierung von Altbäumen entspricht (De Roman et al., 2005; Lang, 2008; Lang et al., 2011a; Lang \& Polle, 2011b). Die Vitalitätsrate war hingegen im Reinbestand höher als im Mischbestand, was auf Konkurrenzeffekte zurückzuführen ist. Die negative Beeinflussung der Buche im unterirdischen Wurzelbereich wurde beispielweise auch durch Beyer et al. (2013) in einem Konkurrenzversuch zwischen jungen Buchen und Eschen beobachtet. Unabhängig davon entsprachen die, in unserer Untersuchung beobachteten Morphotypen, denen der charakteristischen Arten für Buche (Lang, 2008) und es wurden keine nennenswerten Unterschiede zwischen Rein- und Mischbestand beobachtet. Das Auftreten von Inocybe glabris Reinbestand, jedoch nicht im Mischbestand, kann als eher zufälliges Ereignis verstanden werden, da nicht das komplette Wurzelwerk der Buchen untersucht wurden, sondern lediglich eine Teilmenge und daher nicht zweifelsfrei davon ausgegangen werden kann, dass Inocybe glabris nicht an anderer Stelle im 
Wurzelbereich des Mischbestandes auftritt. Zumal die Abundanz dieser Art im Reinbestand im Vergleich zu anderen Arten, wie z.B. Clavulina cristata und Lactarius subdulcis nicht besonders hoch erschien.

An Hand der Stickstoffmarkierung konnten wir zeigen, dass die Wurzeln den Stickstoff aus dem Boden in beiden Beständen aufgenommen haben, da sich die Akkumulation vom Boden über die Rhizosphäre in Richtung der Wurzeln erhöhte. In Bezug auf die Stickstoffakkumulation in den Feinwurzeln konnten wir zudem zeigen, dass sich der zeitliche Verlauf in beiden Beständen ähnlich verhält. Sowohl im Reinals auch im Mischbestand verbleibt das Stickstoffniveau nach einem deutlichen anfänglichen Anstieg konstant erhöht über den Versuchszeitraum. Die aus dem Transport aus dem Boden resultierende Akkumulation in den Feinwurzeln des Reinbestandes ist im Vergleich zu denen des Mischbestandes höher, was sich als Folge der ebenfalls leicht erhöhten Akkumulation von ${ }^{15} \mathrm{~N}$ im Boden interpretieren lässt. Möglicherweise ist die Akkumulation im Reinbestand auf Grund fehlender Nährstoffkonkurrenz durch andere Laubbaumarten erhöht. 


\subsection{Literatur}

Beyer F, Hertel D, Jung K, Fender AC, Leuschner C (2013) Competition effects on fine root survival of Fagus sylvatica and Fraxinus excelsior. For Ecol Manag 302: 1422

Bolte A, Villanueva I (2006) Interspecific competition impacts on the morphologyand distribution of fine roots in European beech (Fagus sylvatica L.) and Norway spruce (Picea abies (L.) Karst.). Eur J For Res 125:15-26

DeRoman M, Claveria V,DeMiguel AM (2005) A revision of the descriptions of ectomycorrhizas published since 1961. Mycol Res 109: 1063-1104

Ellenberg H, Leuschner C (2008) Vegetation Mitteleuropas mit den Alpen. 6. Auflage, Ulmer Verlag, Stuttgart

Ellenberg H, Strutt GK (2009) Vegetation Ecology of Central Europe. $4^{\text {th }}$ Edition, Cambridge Academic Press

Lang C (2008) Diversität in Ektomykorrhizen in verschieden artenreichen Laubbaumbeständen im Nationalpark Hainich (Thüringen). Band 1 Göttinger Forstwissenschaften, Universitätsverlag Göttingen

Lang C, Dolynska A, Finkeldey R, Polle A (2010) Are beech (Fagus sylvatica) roots territorial) For Ecol Manag 260:1212-1217

Lang C, Seven J, Polle A (2011a) Host preferences and differential contributions of deciduous tree species shape mycorrhizal species richness in a mixed Central European forest. Mycorrhiza 21: 297-308

Lang C, Polle A (2011b) Ectomycorrhizal fungal diversity, tree diversity and root nutrient relations in an mixed Central European forest. Tree Physiol 31 (5): 531-538

Lei P, Scherer-Lorenzen M, Bauhus J (2012) The effect of tree species diversity on fine-root production in a young temperate forest. Oecologia 169:1105-1115

Leuschner C, Hertel D, Schmid I, Koch O, Muhs A, Hölscher D (2004) Stand fine root biomass and fine root morphology in old-growth beech forests as a function of precipitation and soil fertility. Plant Soil 258:43-56

McClaugherty CA, Aber JD, Melillo JM (1982) The role of fine roots in the organic matter and nitrogen budgets of two forested ecosystems. Ecology 63(5): 1481-1490 Meinen C, Hertel D, Leuschner C (2009) Root growth and recovery in temperate broad-leaved forest stands differing in tree species diversity. Ecosystems 12:11031116 
Meinen C, Leuschner C, Ryan NT, Hertel D (2009 b) No evidence of spatial root system segregation and elevated fine root biomass in multi-species temperate broadleaved forests. Trees 23: 941- 950

Mühlenberg, M (1993) Freilandökologie 3. Auflage, Uni-Taschenbücher 595, Quelle \& Meyer, Heidelberg

Persson H (1978) Root dynamics in a young scots pine stand in Central Sweden. Oikos 30:508-519

Rewald B, Leuschner C (2009) Belowground competition in broad-leaved temperate mixed forest. Pattern analysis and experiments in a four-species stand. Eur $\mathrm{J}$ Forest Res 128:387- 398)

Sadanandan EK, Sands R (1993) Competition for water and nutrients in forests. Can J For Res 23:1955-1968

Sanantonio D, Hermann RK, Overton WS (1977) Root biomass studies in forest ecosystems. Pedobiologia 17:1-31

Schmid I, Kazda M (2002) Root distribution of Norway spruce in monospecific and mixed stands on different soils. For Ecol Manag 159:37-47

Schulz H, Härtling S, Stange CF (2011) Species-specific differences in nitrogen uptake and utilization by six European tree species. J Plant Nutr Soil Sci 174:28-37 Schutt P, Schuck HJ, Stimm B (1992) Lexikon der Baum- und Straucharten. Nikol Verlagsgesellschaft, Hamburg

Tamm CO (1991) Nitrogen in terrestrial ecosystem. Ecological studies 81, New York Vitousek PM, Horwarth RW (1991) Nitrogen limitation on land and in the sea: how can it occur? Biogeochemistry 13:87-115 
3.Kapitel: C- und N-Fluss in Buche (Fagus sylvatica) und Esche (Fraxinus excelsior) von den Blättern in mykorrhizierte Wurzelspitzen

\subsection{Einleitung}

Der Stofftransport ist einer der wichtigsten Prozesse für das Wachstum von Bäumen und die Aufrechterhaltung des Stoffwechsels in symbiontischen Systemen. In Bezug auf die Mykorrhiza, die Symbiose zwischen Pflanzenwurzeln und Pilzen, sind insbesondere der Transport von Kohlenstoff von Seiten der Pflanze in Richtung des Pilzes sowie der Transport von Stickstoff vom Pilz zur Pflanze von Bedeutung (Marschner \& Dell, 1993; Read \& Perez-Moreno,2003). Die Detektion der Transportprozesse ist durch die Anwendung von stabilen Isotopen möglich und wurde sowohl in Freiland- als auch Laborexperimenten häufig eingesetzt (Philip \& Simard, 2008; Högberg et al., 2007; He et al., 2009).

Die Wälder Mitteleuropas sind potentiell von der Buche (Fagus sylvatica) dominiert (Ellenberg, 1996), die aber häufig mit weiteren Laubbäumen wie z. B. der Esche (Fraxinus excelsior) vergesellschaftet ist. Der Transport von Kohlenstoff und Stickstoff kann zwischen Baumarten variieren. Die spezifische Stickstoffaufnahme verschiedener ökonomisch und ökologisch bedeutsamer Baumarten in Europa, darunter auch Buchen und Eschen, wurde an zweijährigen Pflanzen an Hand von $\mathrm{NH}_{4} \mathrm{NO}_{3}$-Injektion in den Boden in einem Gewächshausversuch untersucht (Schulz et al., 2011). Dabei wurde beobachtet, dass die Stickstoffaufnahme der Wurzeln von Eiche und Buche geringer war als bei Linde und Esche. Zum Beispiel nahmen die Buchen 30,1 $\mu \mathrm{g} \mathrm{NO} / \mathrm{Tag}$ und die Eichen 14,3 $\mathrm{mg} \mathrm{NO} / \mathrm{Tag}$ auf, während die durchschnittliche $\mathrm{NO}_{3}$-Aufnahmerate von Linde und Esche mit 95,7 bzw. 182,4 $\mathrm{NO}_{3}$ $\mu \mathrm{g} / \mathrm{Tag}$ deutlich höher war (Schulz et al., 2011). Die Esche tritt mit der Buche häufig in Gesellschaft auf und es wird erwartet, dass sich ihr Vorkommen unter wärmeren Umweltbedingungen ausbreiten wird (Broadmeadow \& Ray, 2005). Die beiden Baumarten zeigen unterschiedliche Charakteristika in Bezug auf Wachstumsgeschwindigkeit, Streuqualität, Mykorrhizaform und Wurzelanatomie (Grime et al., 1997; Meinen et al., 2009). Freilanduntersuchungen zeigten, dass die Buche eine höhere Anzahl an Wurzelspitzen und eine höhere spezifische Wurzeloberfläche aufweist als die Esche (Meinen et al., 2009). Der Vergleich von Buche und Esche ist interessant, weil sie mit verschiedenen Mykorrhizaformen vergesellschaftet sind. Während die Esche mit der arbuskulären Mykorrhiza, die im Inneren der Wurzelzellen wächst, vergesellschaftet ist, formt die Buche an der 
Oberfläche ihrer Wurzeln eine Symbiose mit Ektomykorrhizapilzen (Smith \& Read, 2008). In Gewächshausversuchen zeigte der Stofftransport von ${ }^{13} \mathrm{C}$ vom Baum in die Mykorrhiza und die im Boden lebenden Tiere eine hohe Anreicherung von $13 \mathrm{C}$ in den Wurzeln beider Baumarten mit maximalen Werten in der Ektomykorrhiza (Eissfeller et al., 2013). Die Pflanzen wurden dazu fünf Monate in einer ${ }^{13} \mathrm{CO}_{2}$ Atmosphäre kultiviert. In einem weiteren Markierungsexperiment im Gewächshaus wurden jungen Buchenpflanzen über eine Vegetationsperiode unter $\mathrm{CO}_{2}$-Atmosphäre kultiviert und zusätzlich mit einer ${ }^{15} \mathrm{~N}-\mathrm{Nährlösung} \mathrm{gegossen.} \mathrm{Dabei} \mathrm{zeigte} \mathrm{sich,} \mathrm{dass}$ die Akkumulation von ${ }^{13} \mathrm{C}$ und ${ }^{15} \mathrm{~N}$ den mykorrhizierten Wurzelspitzen um 40 bis $50 \%$ im Vergleich zu den Feinwurzeln erhöht war (Valtanen et al., 2014). Dies lässt einen größeren Bedarf an Kohlenstoff der Ektomykorrhiza vermuten und wies an Hand von Korrelationen weiter auf einen inversen Fluss von $\mathrm{C}$ und $\mathrm{N}$ zwischen Wurzeln und Ektomykorrhiza hin. Die Bedeutung der Feinwurzeln und der Mykorrhiza in Bezug auf Stoffallokation zeigte sich auch im Freiland. Feinwurzeln spielen eine Schlüsselrolle im Kohlenstoffhaushalt von Wäldern, wobei die Wurzelspitzen dabei eine besondere Bedeutung haben. Ihre primäre Aufgabe ist die Wasser- und Nährstoffaufnahme (Keel et al., 2012). Die an den Wurzelspitzen vergesellschaftete Mykorrhiza spielt auch in Bezug auf Stickstoff eine große Rolle. Die Mykorrhiza ist in der Lage Streu zu zersetzen und die enthaltenen Nährstoffe durch oxidative und hydrolytische Enzyme für die Pflanze verfügbar zu machen (Näsholm et al., 2012)). Die Bedeutung der Mykorrhiza für die Nährstoffmobilisierung wurden von Abuzinadah und Read (1986 a) in einem Versuch mit Kiefern gezeigt, worin Pflanzen, die mit Paxillus involutus, Rhizopogon roseolas und Suillus bovinus vergesellschaftet waren $\mathrm{N}$ aus Proteinquellen nutzen konnten; aber fehlender Mykorrhiza allerdings nicht. Diese Tatsache konnten sie weiterhin an Fichten, Kiefern und Birken, die mit Hebeloma crustuliniforme vergesellschaftet waren, zeigen (Abuzinadah \& Read, 1986 b). In einem Freilandexperiment wurde mit Hilfe von sogenannten "litter-bags" der Transport von ${ }^{15} \mathrm{~N}$ aus Streu in den umgebenden Boden und die Pflanzenwurzeln untersucht und es zeigte sich, dass einzelne Pilze räumlich und zeitlich in ihrer Allokation variieren können (Pena et al., 2013).

Stickstoff wird jedoch nicht nur aus dem Boden über die Wurzeln aufgenommen, sondern auch über oberirdische Pflanzenbiomasse. Wilson (1992) zeigte, dass Fichten die mit $\mathrm{NH}_{4}{ }^{+}$bzw. $\mathrm{NO}_{3}{ }^{-}$-Regenlösungen behandelt wurden, $\mathrm{NH}_{4}{ }^{+}$über die Blätter aufnahmen und die Konzentration in den Blättern mit zunehmender 
Stickstoffkonzentration in der Lösung zunahm. $\mathrm{NO}_{3}{ }^{-}$in der Lösung konnte jedoch nicht von den Pflanzen genutzt werden (Wilson, 1992) In einem Gewächshausversuch konnte gezeigt werden, dass junge Buchen signifikante Mengen an Stickstoff aufnehmen, wenn eine sprühregenartige Deposition künstlich erzeugt wird (Brumme et al., 1992). Obwohl der Großteil des markierten Stickstoffes in den Blättern und im Stamm gemessen wurde, wurden 7-14\% des markierten Stickstoffes in den Wurzeln gefunden, woraus auf einen Transport innerhalb der Pflanzen geschlossen werden kann. An jungen Buchen wurde festgestellt, dass die Akkumulation von Stickstoff in Form von Aminosäuren (Gln bzw. Asp.) über die oberirdische Biomasse einen Rückgang der Wurzelaufnahme zwischen 61\% (Gln) und $79 \%$ (Asp) verursacht (Geßler et al., 1998). Ähnliche Ergebnisse zeigten auch Untersuchungen an jungen Fichten, die mit ${ }^{14} \mathrm{C}$ und ${ }^{15} \mathrm{~N}$ - markierten Aminosäuren über die Zweige behandelt wurden (Geßler et al., 2003). Dabei zeigte sich, dass 22$26 \%$ der Markierung aufgenommen wurden und weiterhin, dass der Transport von Asp $-{ }^{14} \mathrm{C} 30 \%$ schneller erfolgte als im Falle des Gln-eingebetteten radioaktiven Kohlenstoffes. Dies zeigt, dass im Fall des Stickstoffes nicht unerhebliche Konzentrationen von der oberirdischen Biomasse in die unterirdische transportiert werden können. Ob dies auch für die mykorrhizierten Wurzelspitzen zutrifft, wurde bisher nicht überprüft. Ausserdem ist unklar, ob und welche Unterschiede es im Cund N-Transport zwischen Buchen und Eschen gibt. Der vorliegenden Untersuchung lagen folgende Fragen zu Grunde:

Wird in den Wurzelspitzen mehr Kohlenstoff akkumuliert als in den Feinwurzeln und gibt es Unterschiede zwischen Buche und Esche?

Wird in den Wurzelspitzen ebenfalls mehr Stickstoff akkumuliert als in den Feinwurzeln und kommt es zu einer Anreicherung über die Zeit? 


\subsection{Material \& Methoden}

\subsubsection{Untersuchungsgebiet und Auswahl der Pflanzen}

Das Untersuchungsgebiet "Lichte Haardt" ( $\left.51^{\circ} 05^{\prime} 28^{\prime \prime} \mathrm{N}, 10^{\circ} 31^{\prime} 24^{\prime \prime} \mathrm{E}\right)$ befindet sich angrenzend an den Ort Weberstedt in der Nähe der Kreisstadt Mühlhausen im Nationalpark Hainich, Thüringen, Deutschland. Der Hainich ist ein Muschelkalkhöhenzug und bildet das größte zusammenhängende Laubwaldgebiet in Deutschland. Das Gebiet des Nationalparks erstreckt sich über 16.000 Hektar und wird seit rund 40 Jahren nicht mehr bewirtschaftet. Die Jahresmitteltemperatur beträgt $7,5^{\circ}$ Celsius mit einem Jahresniederschlag von $670 \mathrm{~mm}$ (Leuschner et al., 2009). Auf dem Untersuchungsplot stellt die Rotbuche (Fagus sylvatica) die Hauptbaumart dar; mit einer natürlichen Beimischung von Esche (Fraxinus excelsior). Für das Experiment wurden jeweils 25 Pflanzen der Baumarten Buche (Fagus sylvatica) und Esche (Fraxinus excelsior) in einem durch Wanderwege eingerahmten Bestand (51 ${ }^{\circ} 50^{\prime}{ }^{\prime \prime} \mathrm{N} 10^{\circ} 30^{\prime} 20^{\prime \prime} \mathrm{E}$ ) (Abbildung 3.2.1) ausgewählt. 20 Bäume jeder Art wurden als zu markierende Pflanzen und 5 Bäume pro Art als Kontrollpflanzen ausgewählt. Die Bäume hatten ein geschätztes Alter von 3-5 Jahren und eine Höhe zwischen 2-4 m. Die Bäume wurden derart ausgewählt, dass jeweils eine Buche und Esche in möglichst ähnlichen Lichtverhältnissen standen und ein Mindestabstand von $5 \mathrm{~m}$ zwischen ihnen bestand.

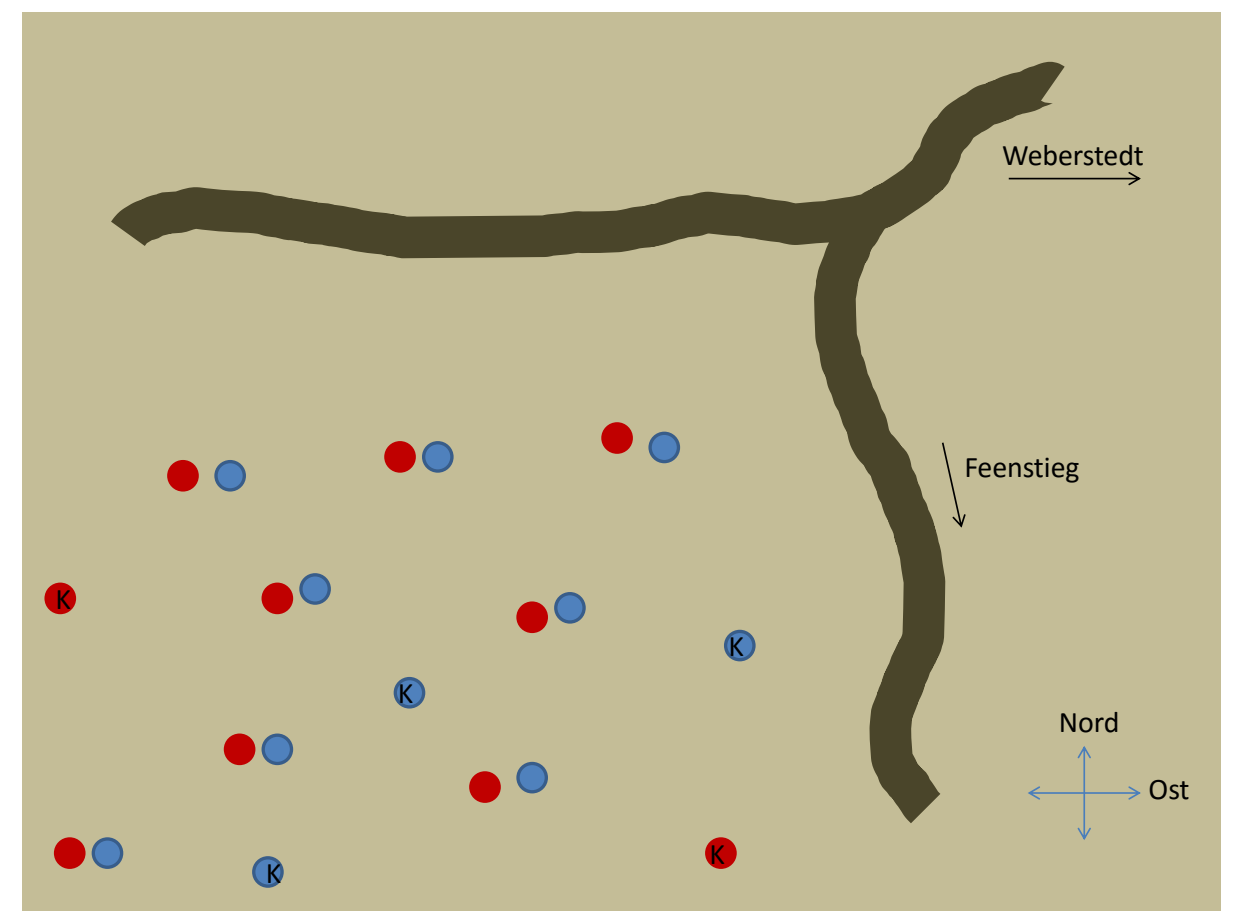

Abbildung 3.2.1: Schematische Darstellung des Untersuchungsgebietes (rot= Buche, blau $=$ Esche, $\mathrm{K}=$ Kontrolle) 
Es wurde eine quadratische Fläche von $1 \times 1 \mathrm{~m}$ mit dem Stamm als Mittelpunkt mit Kunststoffplatten (1 m Länge) abgesteckt und diese $10 \mathrm{~cm}$ in den Boden eingebracht (Abbildung 3.2.2). Die so entstandene Innenfläche durfte nicht betreten werden.

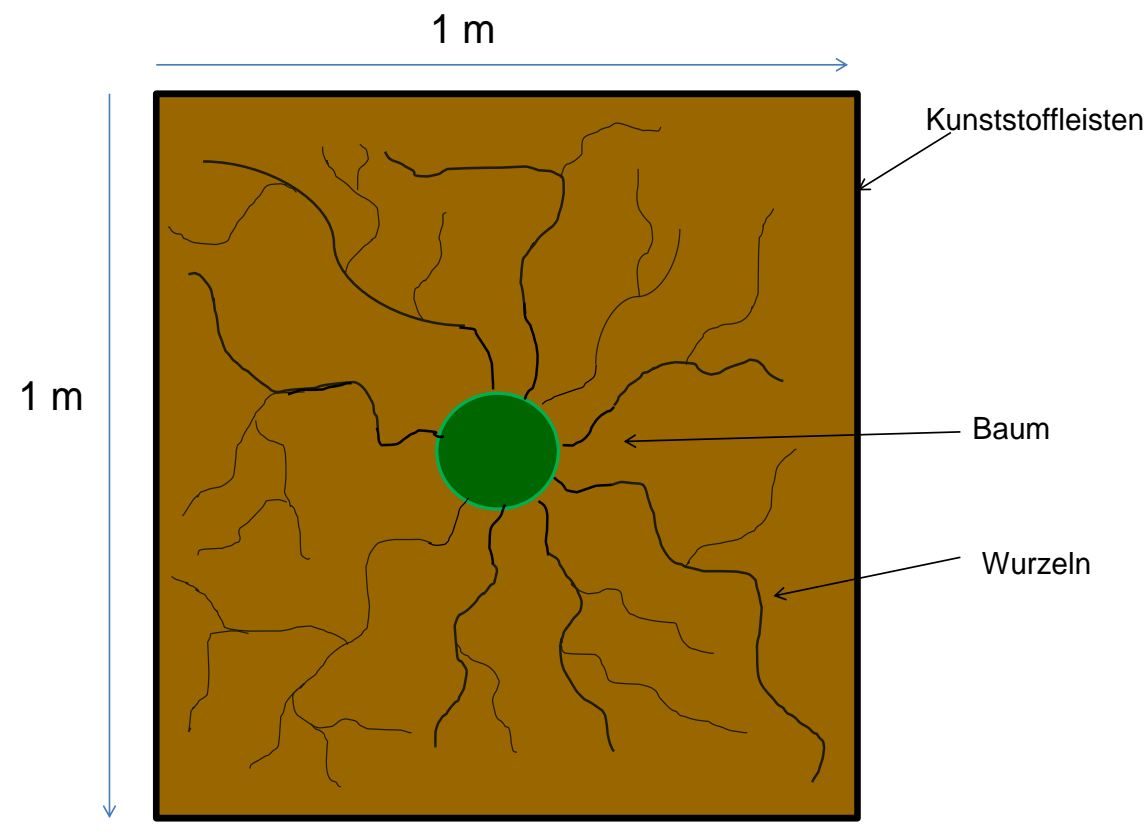

Abbildung 3.2.2: Schematische Darstellung eines Versuchsplots

Bei dem folgenden Aufbau der Holzgestelle zur Befestigung der Plastiktüten (siehe Abschnitt 3.2.2.1) wurde darauf geachtet, dass alle Stützbalken außerhalb des abgesteckten Quadrates installiert wurden. 


\subsubsection{Isotopenmarkierung}

\subsubsection{Kohlenstoffmarkierung}

Zur Vorbereitung des Experimentes wurden Holzgestelle, die die Bäume einrahmten, gefertigt. Die Begasung der Bäume mit ${ }^{13} \mathrm{CO}_{2}$ erfolgte in einem abgeschlossenen System, in dem die Bäume jeweils von einem großen Plastikschlauch umhült wurden und von der Außenluft abgeschlossen wurde. Das System wurde von den Holzkonstruktionen gehalten.

Für die Durchführung der ${ }^{13} \mathrm{C}$-Markierung wurden wegen der Pflanzenhöhe von bis zu 4 m Hilfskonstruktionen (Abbildung 3.2.3) benötigt. Dazu wurden aus Fichten/Tannen-Latten (24 x 48 mm, 3 m lang, OBI, Wermelskirchen, Deutschland) Tore gebaut. Ein Teil der Latten wurden auf eine Länge von 1,5 m gekürzt und dienten als Dachlatte. Daran wurden an beiden Enden 3 m lange Latten mit Nägeln als Seitenstützen befestigt. Je nach Pflanzenhöhe wurden die Latten durch Annageln weiterer Latten verlängert. Am Boden wurde die Konstruktion durch 2 angenagelte, kürzere Seitenlatten, die schräg von langen Seitenlatten in den Boden führten, zusätzlich gestützt.

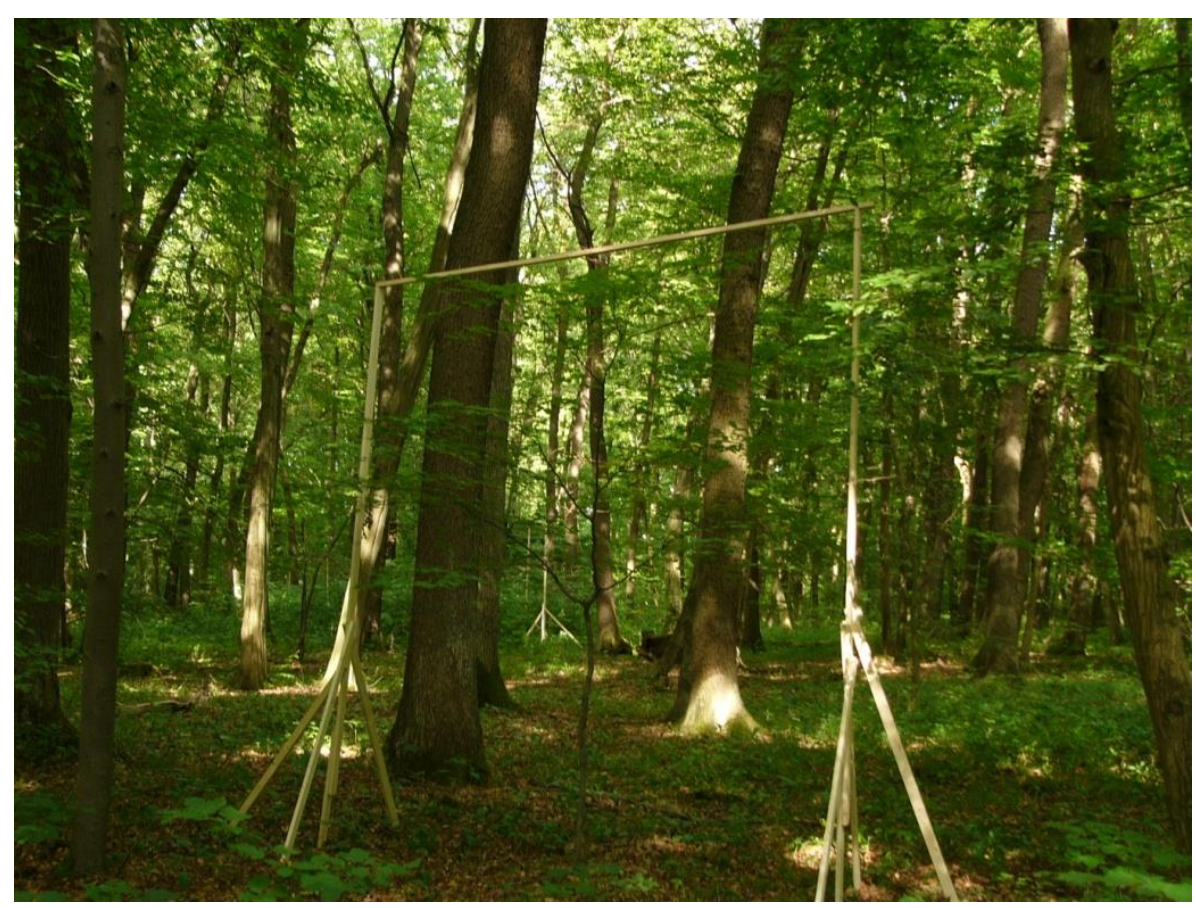

Abbildung 3.2.3: Holzkonstruktion zur Installation des Kunststoffschlauches (Fotos: S. Zieger)

Für die Konstruktion des Kunststoffschlauchs zur Begasung wurde eine PE (Polyethylen)-Folie, die in Gewächshäusern und Treibhäusern eingesetzt wird, 
verwendet. Die Folie ist mit einer Fläche von $4 \mathrm{~m}$ Breite und $50 \mathrm{~m}$ Länge (Polydress Plastic $\mathrm{GmbH}$, Michelstadt, Deutschland) erhältlich. Die 0,08 mm starke Folie wurde auf Grund ihrer Reißfestigkeit, UV-Stabilität, Temperaturunempfindlichkeit und Dauerhaftigkeit für den Freilandeinsatz ausgewählt. Die Folie wurde von Folien Bernhardt, Dreieich, Michelstadt mit einer Schweißnaht versehen und als Schlauch nach Göttingen geliefert. Für jeden der 2 bis $4 \mathrm{~m}$ hohen Bäume wurde ein $5 \mathrm{~m}$ langes Schlauchstück von der Rolle abgetrennt. Die beiden offenen Schlauchseiten wurden mit doppelseitigem Klebeband (PERFECTA CLASSIC, $50 \mathrm{~mm}$ breit, Eugen Nölle, noelle group $\mathrm{GmbH}$ \& Co KG, Sprockhövel, Deutschland) versehen. Zur Vorbereitung des Freilandexperimentes wurde nur die obere der beiden Schlauchseiten verklebt. Dabei wurde darauf geachtet, dass beide Flächen lückenlos miteinander verklebt wurden. Entstehende Löcher bergen die Gefahr des Verlustes von ${ }^{13} \mathrm{CO}_{2}$-Gas in die Umgebungsluft während des Markierungsexperimentes. Die verbleibende untere Seite wurde erst nach Installation der Ballons um die Bäume verschlossen, da der Baumstamm in das Konstrukt integriert werden musste. Zum Transport in den Wald wurden die einzelnen Schläuche eingerollt. Für die Durchführung der ${ }^{13} \mathrm{C}$ - Markierung wurden die an den Dachlatten befestigten und Schläuche ausgerollt und über den jeweiligen Baum gestülpt (Abbildung 3.2.4).

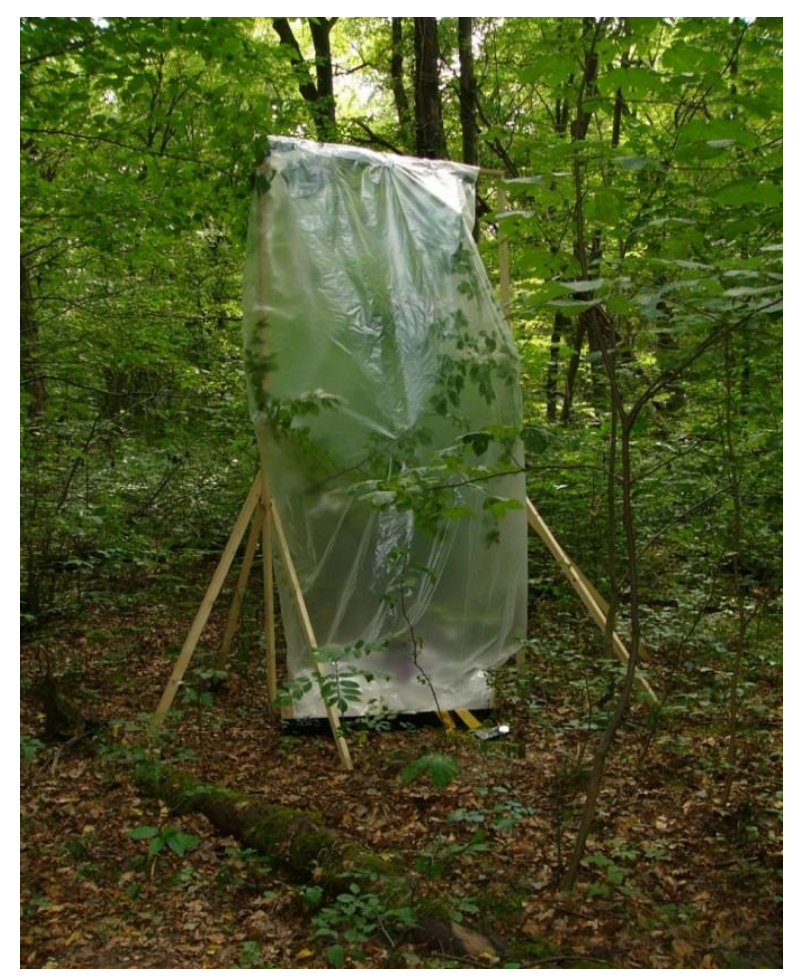


Die untere, mit doppelseitigem Klebeband versehene Öffnung wurde in horizontaler Richtung zur Hälfte - bis zum Stamm - geschlossen. Da der Ballon bis auf den Erdboden reichte, wurde eine Fläche geschaffen, auf der weitere Hilfsmittel installiert werden konnten. Neben einem Kühlakkumulator, der als Kühlfalle für sich bildendes Kondenswasser diente, wurden dort auch die Gefäße, die für die Markierung notwendig waren, platziert. Die mit einer Carbonatlösung (siehe unten) gefüllten 500 ml-Kunststoffflaschen wurden mit Duct tape (TESA, Hamburg, Deutschland) in den Kunststoffschüsseln befestigt, um ein Umkippen der Flaschen zu verhindern. Diese Konstruktion wurde ausgewählt, da so eine größere und damit eine sicherere Standfläche im Vergleich zu den Carbonat-gefüllten Flaschen allein erzielt wurde. Die Schüsseln wurden auf der - durch das Verkleben der beiden Ballonseiten entstandenen- Fläche vor den Stamm gestellt und der Deckel der Flaschen entfernt. Zwischen Stamm und Schüssel wurde ein über Ladeakkumulatoren betriebener Ventilator (MagLev DC 12 V (4,8-12 W), Sunon, Fresnes Cedex, France) platziert (Abbildung 3.2.5).

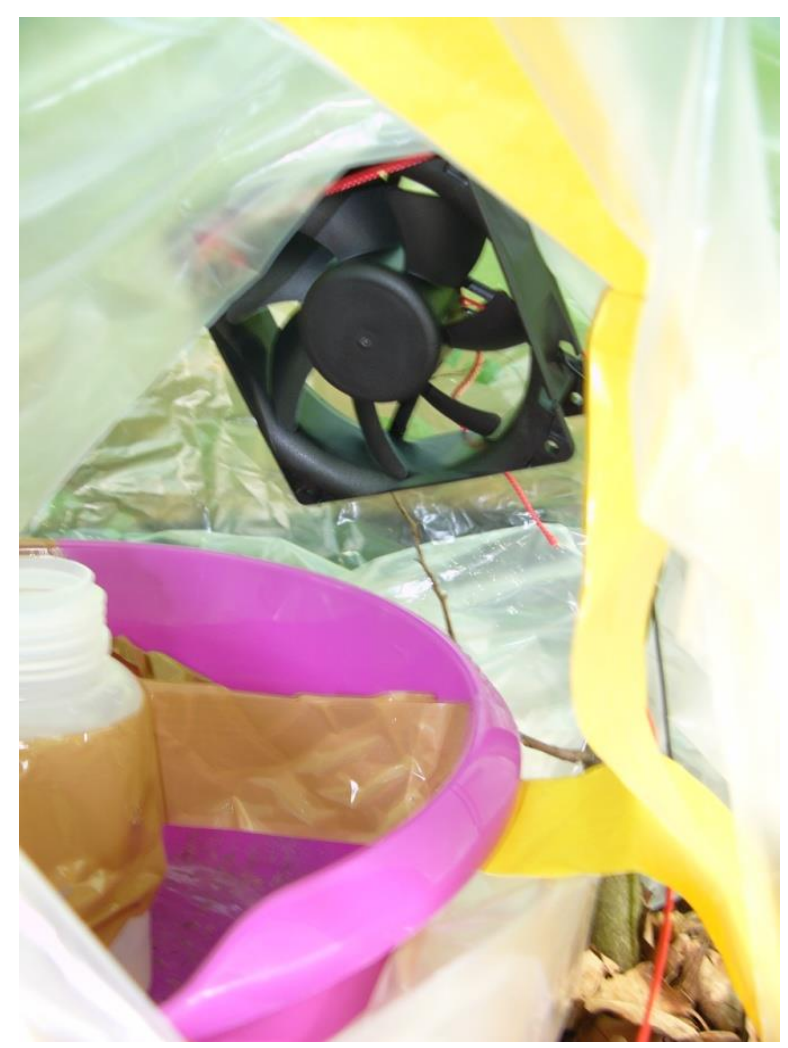

Abbildung 3.2.5: Befestigung des Ventilators im Schlauch 
Der Ventilator wurde in Höhe des Gefäßrandes der Markierungslösung am Baum befestigt. Durch den Ventilator wurde das entstehende Gas gleichmäßig verwirbelt, sodass dieses auch die oberen Blätter des Baumes erreichte. Die Leitungskabel des Ventilators wurden am Stamm entlang aus dem Ballon geführt und die restliche offene Seite des Schlauches auf Bodenhöhe verklebt. Dabei wurde darauf geachtet, dass insbesondere der Stamm dicht in der Kunststofffolie verbunden wurde.

Zur ${ }^{13} \mathrm{CO}_{2}$-Markierung wurde mit einer $100 \mathrm{ml}$ - Aufziehspritze (bacuplast, Remscheid, Deutschland) mit aufgesetzter Metallkanüle $60 \mathrm{ml} 5 \mathrm{M} \mathrm{H}_{2} \mathrm{SO}_{4}$ (Merck, Darmstadt, Deutschland) aufgezogen, auf Höhe der Kunststoffflasche von außen durch die Folie gestochen und die Schwefelsäure in eine Lösung aus $100 \mathrm{ml}$ destilliertem Wasser und $6,85 \mathrm{~g}$ Natriumcarbonat $\left(99 \%{ }^{13} \mathrm{C}\right.$ ) (Cambridge Isotope Laboratories, Tewksbury, MA, USA) gegeben. Durch mehrmaliges Aufziehen und Abgeben der Mischung mit der Spritze wurde die Lösung aus Schwefelsäure und Natriumcarbonat vermischt.

Aus der chemischen Reaktion von Natriumcarbonat mit Schwefelsäure entsteht Natriumsulfat, Wasser und Kohlendioxid. Der Kohlenstoff im Carbonat bestand hier zu $99 \%$ aus ${ }^{13} \mathrm{C}$-Atomen, sodass $99 \%$-iges ${ }^{13} \mathrm{C}$-Kohlendioxid entstand.

Das Entweichen des Gases unter Blasenbildung ließ erkennen, dass die chemische Reaktion ablief. Das beim Einspritzen der Säure entstandene Loch in der Folie wurde nach dem Entfernen der Spritze sofort mit reißfestem Klebeband (duct tape, TESA, Hamburg, Deutschland) verschlossen und der Ventilator an den Akkumulator angeschlossen. Um die Verwirbelung des Gases zu unterstützen, wurde die Folie für einen Moment zusätzlich per Hand bewegt.

Ungefähr zehn Minuten nach Beginn des Experimentes wurde mit einer Aufzugspritze (Typ nach DIN 13098, bacuplast, Remscheid, Deutschland) eine Gasprobe genommen und in einen Exetainer (Labco Limited, Ceredigion, Großbritannien), d. h. eine mit einem Septum verschlossene $12 \mathrm{ml}$ - Glasröhre überführt. Das entstandene Loch in der Schlauchfolie wurde sofort mit reißfestem Klebeband (duct tape, TESA, Hamburg, Deutschland) verschlossen. Fünf Stunden nach Beginn der Begasung wurde eine weitere Gasprobe entnommen und danach 
die Folie von den Pflanzen entfernt. Die Gasproben wurden von der AG Kuzyakov, Bodenkunde der Universität Göttingen, verwahrt.

\subsubsection{Stickstoffmarkierung}

Für die Stickstoffmarkierung wurde eine $2 \mathrm{M} \mathrm{Ca}\left({ }^{15} \mathrm{NO}_{3}\right)_{2}$-Lösung verwendet. Dazu wurden $36 \mathrm{~g}$ Calciumnitrat (99,23 atom\%) (Campro Scientific GmbH, Berlin, Deutschland) in $1200 \mathrm{ml}$ destilliertem Wasser gelöst. Jeweils 9,7 ml Lösung wurde in 12ml Glas- Exetainer (Labco Limited, Ceredigion, Großbritannien) gefüllt.

An den Buchen wurden drei Blätter an einem Seitenzweig ausgewählt. Dazu wurde die Krone jedes einzelnen Baumes betrachtet und visuell in drei Höhenstufen „obere Krone“, „Mittelkrone“ und „untere Krone“ eingeteilt. In jeder der drei Stufen wurden jeweils ein Seitenzweig ausgewählt. Die Blätter wurden quer zur Hauptnarbe eingerissen, um die Aufnahme der Markierungslösung über die Wunde zu beschleunigen. Die Blätter wurden längs zusammengerollt und gemeinsam in den, mit Markierungslösung gefüllten, Exetainer gebracht. Um das Eindringen von Flüssigkeiten von außen oder Verschmutzungen sowie das Auslaufen der Markierungslösung zu verhindern, wurden die Exetainer mit Parafilm („M“, Bemis Company Inc., Neenah, Wisconsin, USA) verschlossen. Die zugehörigen Exetainerdeckel wurden nicht verwendet, da sie keinen ausreichenden Durchlass für die Blattstengel bieten konnten. Pro Baum wurden drei Exetainer angebracht, wobei darauf geachtet wurde, dass sich die markierten Blätter in verschiedenen Kronenhöhen (obere Krone, Kronenmitte und untere Krone) befanden und die verwendeten Zweige nicht zu nah nebeneinander lagen. Dadurch sollte eine gleichmäßige Markierung erzielt werden. Bei den Eschen wurde die gleiche Prozedur durchgeführt, wobei pro Exetainer jeweils drei Fiederblättchen verwendet wurden.

Für den Fall, dass auf Grund von Windbewegung oder Wildverbiss Exetainer von den Bäumen gerissen würden, wurden über die Exetainer Plastiktüten gestülpt und mit Gummiband verschlossen, um eine Kontamination des Bodens zu verhindern (Abbildung 3.2.6). Ebenso sollte eine Verdünnung der Markierungslösung durch Flüssigkeiten von außen (Regen, Tau) vermieden werden. Daher wurde der Rand der Plastiktüten über die Exetainer gelegt, damit Wasser gegebenenfalls über den Beutel ablaufen konnte. Die Plastiktüten wurden mit Gefriertütenverschlussdraht (Toppits, Cofresco Frischhalteprodukte $\mathrm{GmbH} \&$ Co.KG, Minden, Deutschland) an dickeren Ästen des Baumes oder an der Holzkonstruktion befestigt. 


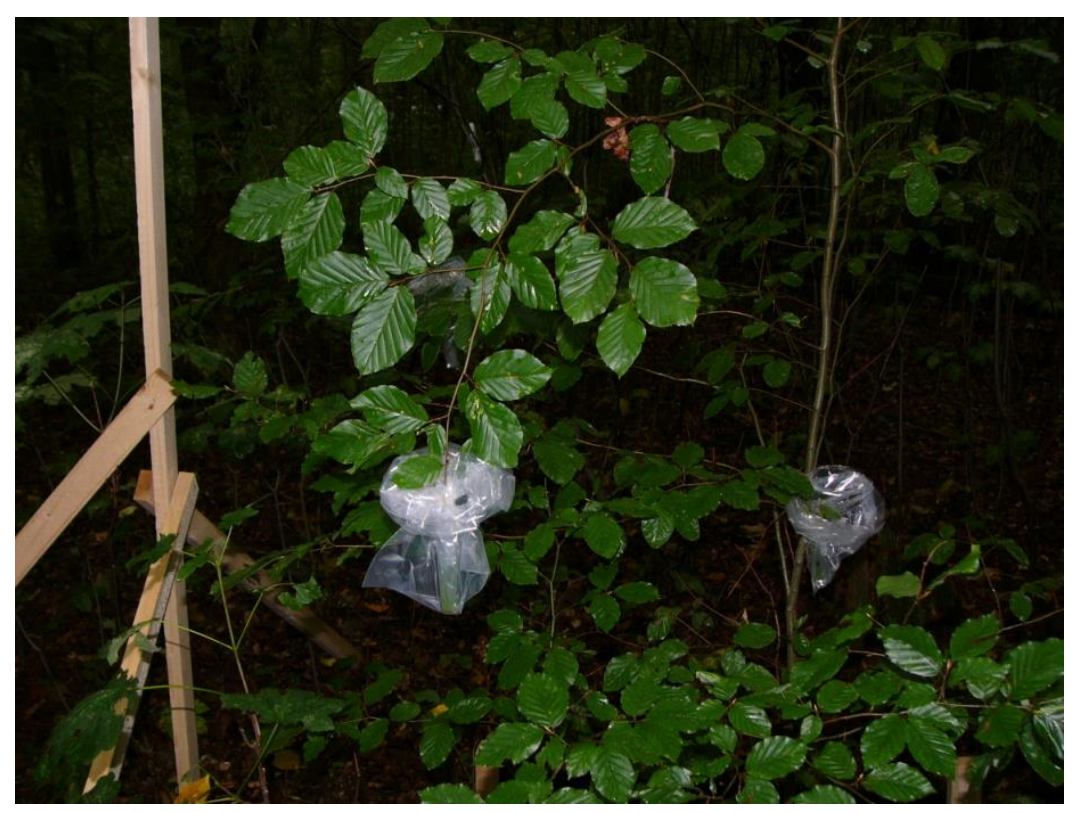

Abbildung 3.2.6: Stickstoffmarkierung an einer Buche

Während einer trockenen Wetterlage erfolgte die Stickstoffmarkierung von Samstag, den 13.08. bis Montag, den 15.08.2011. An diesen Tagen betrug die Niederschlagsmenge im Versuchsgebiet $0 \mathrm{~mm}$, die Temperatur lag zwischen 17 und $23^{\circ}$ Celsius mit einer relativen Luftfeuchte von 60- $69 \%$ (Tabelle 3.2.1;Daten zur Verfügung gestellt von AG Leuschner, Universität Göttingen). Am 13.08. wurden 8 Buchen und 8 Eschen markiert. Am 14.08. und 15.08. wurden jeweils 4 Buchen und 4 Eschen markiert, sodass insgesamt 16 Bäume pro Baumart markiert wurden. Die unterschiedliche Anzahl an Bäumen war auf Grund der nachfolgenden Kohlenstoffmarkierung notwendig. Die markierte Lösung verblieb jeweils 72 Stunden an den Bäumen und wurde danach abgenommen.

Die ${ }^{13} \mathrm{CO}_{2}$-Pulsmarkierung wurde im Anschluss an die ${ }^{15} \mathrm{~N}$-Markierung vom 19.- 21. August 2011 für jeweils 5 Stunden pro Baum durchgeführt. Das Stickstoffmarkierungsexperiment an den gleichen Bäumen wurde zuvor in der Zeit vom 16. - 18. August 2011 durchgeführt. Aus organisatorischen Gründen wurde an den drei Markierungstagen eine unterschiedliche Anzahl an Bäumen mit ${ }^{13} \mathrm{CO}_{2}$ behandelt. Am 16.08. wurden vier Buchen und vier Eschen markiert. Am 17.8. wurden 8 Buchen und 8 Eschen markiert. Am 18.08. erfolgte die Markierung wiederum an vier Buchen und vier Eschen. Vom 16.08. bis zum 18.08.2011 wurden 
während der Stickstoffmarkierung Photosyntheseraten an ausgewählten Buchen und Eschen mit einem LCpro+ Photosynthesesystem (ADC BioScientificLimited, Hertfordshire, UK) durchgeführt. Die Wetterdaten an Markierungstagen wurden von der AG Leuschner, Universität Göttingen zur Verfügung gestellt (Tabelle 3.2.1).

Tabelle 3.2.1: Meteorologische Daten an den Markierungstagen ( $\mathrm{MW}=$ Mittelwert, $\mathrm{STA}=$ Standardabweichung, PAR = photosynthetisch aktive Strahlung)

\begin{tabular}{|l|l|l|r|r|r|r|r|r|}
\hline & Datum & Zeitraum & $\begin{array}{r}\text { PAR } \\
\mu m o l / m^{2} s e c\end{array}$ & $\begin{array}{r}\text { Globalstrahlung } \\
\text { Watt/m }{ }^{2}\end{array}$ & $\begin{array}{c}\text { Wind } \\
\mathrm{m} / \mathrm{s}\end{array}$ & $\begin{array}{c}\text { Regen } \\
\mathrm{mm}\end{array}$ & $\begin{array}{c}\text { Temperatur } \\
{ }^{\circ} \text { Celsius }\end{array}$ & $\begin{array}{c}\text { Luftfeuchte } \\
\%\end{array}$ \\
\hline \multirow{3}{*}{ MW } & 16.8 .11 & $0-24 \mathrm{Uhr}$ & 278,88 & 140,66 & 1,51 & 0 & 17,0 & 69,4 \\
& 17.8 .11 & $0-24 \mathrm{Uhr}$ & 456,97 & 229,30 & 1,85 & 0 & 20,2 & 62,4 \\
& 18.8 .11 & $0-24 \mathrm{Uhr}$ & 432,35 & 216,21 & 1,61 & 0 & 23,0 & 59,7 \\
\hline \multirow{2}{*}{ STA } & 16.8 .11 & $0-24 \mathrm{Uhr}$ & 352,3 & 177,0 & 0,9 & 0 & 2,4 & 8,1 \\
& 17.8 .11 & $0-24 \mathrm{Uhr}$ & 555,8 & 279,9 & 0,8 & 0 & 3,4 & 16,7 \\
& 18.8 .11 & $0-24 \mathrm{Uhr}$ & 481,3 & 241,5 & 0,7 & 0 & 3,5 & 8,1 \\
\hline
\end{tabular}

\subsubsection{Ernte}

1, 5, 10, 20 und 60 Tage nach der ${ }^{13} \mathrm{C}$-Markierung wurden jeweils 4 Buchen und 4 Eschen und ein unbehandelter Kontrollbaum pro Art geerntet. Dazu wurde der Stamm an der Erdoberfläche gekappt und die Blätter zur Isotopenbestimmung abgenommen. Die Blätter wurden gemischt und von der AG Kuzyakov (Bodenkunde Universität Göttingen) weiter analysiert. Vom unteren Stammende wurden die untersten zwei Zentimeter abgetrennt und der Rest des Stammes in kleinere Stücke zerteilt. Die Bodenstreu wurde aus dem abgesteckten Bereich um den Baum abgetragen (AG Scheu) und danach jeweils zwei Bohrkerne (Durchmesser $5 \mathrm{~cm}$, Tiefe $10 \mathrm{~cm}$ ) von zwei beteiligten AGs entnommen (AG Gleixner, AG Kuzyakov). Danach wurden die verbleibenden Wurzeln des Baumes mit einem Spaten ausgegraben und in einer Plastiktüte nach Göttingen transportiert (AG Polle).

Der Boden wurde vorsichtig von den Wurzeln entfernt und die Grob- und Feinwurzeln voneinander getrennt. Frischgewichte vom, an die Wurzeln angrenzenden, Stammstück sowie von Grob- und Feinwurzeln wurden bestimmt. Grobwurzeln wurden zur Bestimmung des Wassergehaltes bei $60^{\circ} \mathrm{C}$ für 1 Woche im Trockenschrank (Memmert GmbH \& Co.KG, Schwabach, Deutschland) getrocknet. Die Feinwurzeln wurden in flüssigem Stickstoff eingefroren und bei $-20^{\circ} \mathrm{C}$ bis zur 
Analyse der Mykorrhizen verwahrt und im Anschluss das Trockengewicht nach 7 Tagen bei $60^{\circ} \mathrm{Celsius}$ bestimmt. Das abgenommene Stammstück wurde in Rinde und Holz geteilt und bei $60^{\circ}$ Celsius eine Woche getrocknet. Rinde und Holz wurden nach dem Trocknen gemahlen (MM 2000, Retsch, Haan, Deutschland).

\subsubsection{Mykorrhizauntersuchung}

\subsubsection{Ektomykorrhiza an Buche}

Zur Bestimmung der Mykorrhizierungsrate und der Vitalitätsrate der Buche wurden 1000 Spitzen der Feinwurzeln unter einem Stereomikroskop (M 205 FA, Leica, Wetzlar, Deutschland) gezählt. Dazu wurden kleine Mengen (2-3 Wurzelstücke pro Portion) aus dem Gefrierlager (-20 Celsius) in einer Petrischale unter dem Mikroskop ausgezählt, bis 1000 Wurzelspitzen erreicht waren. Da die Feinwurzeln (Durchmesser $<2 \mathrm{~mm}$ ) bei der Ernte grob zerschnitten und vor dem Einfrieren vermischt wurden, erfolgte die Auszählung der mykorrhizierten, trockenen und nichtmykorrhizierten Wurzelspitzen an homogenen Mischproben.

In der Petrischale wurden den Wurzelstücken wenige Tropfen Wasser zugegeben, um die Erkennbarkeit der Wurzelspitzenmorphologie zu erleichtern. Es wurde darauf geachtet, möglichst wenig Wasser zuzugeben, um eine Auswaschung von ${ }^{13} \mathrm{C}$ bzw. ${ }^{15} \mathrm{~N}$ zu verhindern. Unter dem Binokular wurde die Wurzel betrachtet und jeweils die Anzahl der nicht-mykorrhizierten, mykorrhizierten und trockenen Wurzelspitzen notiert. Als mykorrhizierte Wurzeln wurden Wurzelspitzen von vitaler Erscheinung und Verbindung mit einem Morphotypen bezeichnet. An den trockenen Wurzelspitzen war es schwierig optisch festzustellen, ob sie mit einem Ektomykorrhizapilz besiedelt waren oder nicht, sodass trockene Wurzelspitzen unabhängig von einer Verbindung mit einem Pilz angesprochen wurden.

Während des Auszählens wurden von jedem Baum Proben der trockenen und mykorrhizierten Wurzelspitzen für die Isotopenbestimmung entnommen und gefriergetrocknet.

Die Mykorrhizierungsrate der Wurzelspitzen (WS) wurden an Hand folgender Formeln berechnet: 
Mykorrhizierungsrate (\%)

$=\frac{\text { Anzahl mykorrhizierter WS }}{\text { Anzahl nicht-mykorrhizierter WS + mykorrhizierter WS }}{ }^{*} 100$

3.2.4.2 Arbuskuläre Mykorrhiza an Esche

Ein Aliquot der Eschenfeinwurzeln wurde zur Analyse der arbuskulären Mykorrhiza bei der Ernte in Ethanol gelagert. Um die pilzlichen Strukturen in den Eschenfeinwurzeln erkenntlich zu machen, wurden sie mit Lactophenol (Merck, Darmstadt, Deutschland) der Methode von Schmitz et al. (1991) eingefärbt. Dazu wurden die Eschenwurzeln mit einer 10\%igen Kalilauge $(\mathrm{KOH})$ für 50 Minuten bei $90^{\circ} \mathrm{C}$ in einem Thermoblock entfärbt. Nach Waschen mit destilliertem Wasser wurden die Feinwurzeln für 15 Minuten in 3,7\%iger Salzsäure bei Raumtemperatur angesäuert. Nach weiterem Waschen mit destilliertem Wasser wurden die Feinwurzeln bis zu 2 Minuten in einer Lactophenol-Lösung (1 $\mathrm{g} / \mathrm{l}$ destilliertes Wasser) eingefärbt. Überschüssiger Farbstoff wurde entfernt, indem die Feinwurzeln danach 60 Minuten in einer sauren Glycerinlösung $(50 \mathrm{ml}$ Glycerin, $45 \mathrm{ml}$ destilliertes Wasser, $5 \mathrm{ml}$ 1\%ige Salzsäure) bei Raumtemperatur inkubiert wurden. Bis zur Analyse wurden die Wurzeln in 50\%-igem Glycerin inkubiert.

Die Auszählung der arbuskulären Mykorrhiza erfolgte nach der "magnified intersection method" nach McGonigle et al. (1990), bei der die Mykorrhizierung der Wurzel an Hand ihrer Anzahl an Hyphen, Vesikel und Arbuskeln charakterisiert wird. Pro Pflanze wurden 3 Feinwurzeln, ausgehend von der Wurzelspitze in Richtung der Grobwurzel am Mikroskop (Axioplan inklusive Digitalkamera AxioCam und Software Axiovision, Zeiss, Oberkochen, Deutschland) ausgezählt. Die Kolonisierung mit Hyphen, Vesikeln und Arbuskeln wurde an Hand folgender Formeln berechnet. 
a) Kolonisierungsrate Hyphen (\%)

$=\frac{\text { Summe an Abschnitten mit Hyphe }}{\text { Summe an Abschnitten mit Wurzel }}{ }^{*} 100$

b) Kolonisierungsrate Arbuskel (\%)

$=\frac{\text { Summe an Abschnitten mit Arbuskel }}{\text { Summe an Abschnitten mit Wurzel }}^{*} 100$

c) Kolonisierungsrate Vesikel (\%)

$=\frac{\text { Summe an Abschnitten mit Vesikel }}{\text { Summe an Abschnitten mit Wurzel }}{ }^{*} 100$

Für die Isotopenbestimmung der Wurzelspitzen der Esche wurden nicht die eingefärbten Wurzelspitzen verwendet, sondern separat eingefrorene, unbehandelte Proben verwendet. Diese Proben wurden, wie die Buchenwurzeln unter dem Binokular betrachtet und entsprechend nach vitaler und trockener Erscheinung entnommen und gefriergetrocknet.

\subsubsection{Isotopenbestimmung}

Die Isotopensignatur wurde in der Rinde, Feinwurzel und in den Wurzelspitzen ermittelt. Zur Vorbereitung der Messung wurden Rinde, trockene und mykorrhizierte Wurzelspitzen gefriergetrocknet (P4K-S, Piatkowski Forschungsgeräte-Vertrieb, München, Deutschland) und die Feinwurzeln im Trockenschrank getrocknet. Die Rinden- und Feinwurzelproben wurden gemahlen (Retsch MM 2000, Haan, Deutschland). Durch das Mahlen wurden homogene Mischproben erzeugt, aus denen die Messproben entnommen wurden. Auf die Homogenisierung der Wurzelspitzen wurde verzichtet, da die Proben komplett in Zinnkapseln (4x6 mm, HEKAtech, Wegberg, Deutschland) eingewogen wurden. Die Isotopenbestimmung wurde mit einem Isotopenmassenspektrometer Delta plus (Finnigan MAT, Bremen, Deutschland) und einem Elementaranalysator NA 1500 (Fisons-Instruments, Rodano, Milano, Italien) im KOSI (Kompetenzzentrum Stabile Isotope) des BüsgenInstitutes der Universität Göttingen durchgeführt.

Die Isotopensignaturen wurden mit folgenden Formeln berechnet: 
a) Isotopensignatur Kohlenstoff $(\partial \% 0))$

$$
\partial{ }^{13} \mathrm{C}(\%)=\frac{\text { R Probe }- \text { R Standard }}{\text { R Standard }} * 1000
$$

mit $R=$ Verhältnis des schweren Isotops $\left({ }^{14} \mathrm{C}\right)$ zu leichtem Isotop $\left({ }^{13} \mathrm{C}\right)$

und Kohlenstoff (Belemnite) aus der PeeDee-Formation (V-PDB) als Standard (Kendall \& Caldwell, 1998)

b) Isotopensignatur Stickstoff (atom\%)

$$
\operatorname{AP}^{15} \mathrm{~N}(\text { atom } \%)=\frac{15 \mathrm{~N}}{(15 \mathrm{~N}+14 \mathrm{~N})}{ }^{*} 100
$$

mit $R=$ Verhältnis des schweren Isotops $\left({ }^{15} \mathrm{~N}\right)$ zu leichtem Isotop $\left({ }^{14} \mathrm{~N}\right)$

und Luftstickstoff als Standard

c) Isotopensignatur Stickstoff (Atom\%excess)

APE ${ }^{15} \mathrm{~N}$ (atom\%excess) $=\frac{15 \mathrm{~N}}{(15 \mathrm{~N}+14 \mathrm{~N})}$ markierte Probe $-\frac{15 \mathrm{~N}}{(15 \mathrm{~N}+14 \mathrm{~N})}$ Kontrolle $) * 100$ 


\subsubsection{Statistik}

Die statistische Auswertung des Experimentes erfolgte mit Microsoft Excel (Windows, Redmond, USA), OriginPro 8.5 (OriginLab Corporation, Northampton, MA, USA) und StatgraphicsPlus (Statgraphics Centurion, Warrenton, Virginia, USA). Die Daten wurden an Hand eines Shapiro-Wilk beziehungsweise Kolmogorov-Smirnov-Test auf Normalverteilung getestet. Für eine ANOVA wurden Zwei-Wege-Tests auf Grundlage von Baumart und Erntetag als Faktoren verwendet. Student's t-Test wurde mit Hilfe von Microsoft Excel (Windows, Redmond, USA) berechnet. Daten, die keine Normalverteilung aufwiesen, wurden an Hand des nicht-parametrischen KruskalWallis-Test analysiert. 


\subsection{Ergebnisse}

\subsubsection{Isotopenmarkierung}

Die Markierung mit Stickstoff wurde drei Tage vor der Kohlenstoffmarkierung durchgeführt. Nach der Inkubation wurde die Stickstoffmarkierung von den Bäumen abgenommen und es folgte eine 5-stündige Begasung mit ${ }^{13} \mathrm{CO}_{2}$. Die im Folgenden angegebenen Erntetage 1, 5, 10, 20 und 60 beziehen sich auf den Zeitpunkt nach der Kohlenstoffmarkierung. Bei den Kontrollen (Tag 0)handelt es sich um die nicht markierten Bäume.

Buchen und Eschen unterschieden sich signifikant in der Trockenmasse ihrer Feinwurzeln (Tabelle 3.3.1). Die für die Markierung verwendeten Buchen hatten im Mittel eine Feinwurzeltrockenmasse von 3,79 \pm 0,81 $\mathrm{g}$ und die verwendeten Eschen eine Feinwurzeltrockenmasse von 1,99 \pm 0,21 g. Demnach war die Trockenmasse der Feinwurzeln der Buchen knapp doppelt so groß wie die der Eschen. Die Trockenmasse der Grobwurzeln der Buchen betrug 3,91 $\pm 0,55 \mathrm{~g}$ und die der Eschen 4,33 $\pm 0,78 \mathrm{~g}$, woraus sich kein Unterschied zwischen den Baumarten ergab. Die Buchen waren zu 98,58 \% \pm 0,49 mykorrhiziert, während in der Esche zu 52,61 \% \pm 1,75 pilzliche Strukturen gefunden wurden.

Tabelle 3.3.1: Feinwurzel- und Grobwurzelbiomasse und Mykorrhizierungsgrad; Daten zeigen Mittelwerte \pm SE, $p$-Werte (einfache ANOVA)

\begin{tabular}{|l|l|l|l|}
\hline Kompartiment & Buche & Esche & $\mathrm{P}$ \\
\hline Feinwurzel & $3,79 \pm 0,81 \mathrm{~g}$ & $1,99 \pm 0,21 \mathrm{~g}$ & 0,035 \\
\hline Grobwurzel & $3,91 \pm 0,55 \mathrm{~g}$ & $4,33 \pm 0,78 \mathrm{~g}$ & 0,829 \\
\hline $\begin{array}{l}\text { Mykorrhizierungs } \\
\text {-grad }(\%)\end{array}$ & $98,58 \% \pm 0,49$ & $52,61 \pm 1,75$ & 0,000 \\
\hline
\end{tabular}

\subsubsection{Kohlenstoffmarkierung in Rinde, Feinwurzel und vitalen Wurzelspitzen von} Buche und Esche

Die natürliche Signatur von $\partial^{13} \mathrm{C}$ in der Rinde war in beiden Baumarten mit $-33,9 \%$ in der Buche und $-33,8 \%$ in der Esche identisch. Einen Tag nach der Markierung

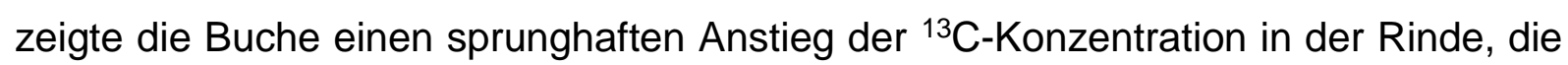
daraufhin wieder deutlich sank und dann im Zeitverlauf relativ konstant blieb (Abbildung 3.3.1). Im Vergleich dazu war die Anreicherung in der Rinde der Esche über den Zeitraum von 60 Tagen niedriger, wenn auch hier ein deutlicher Unterschied zwischen Tag 0 und Tag 1 erkennbar war. Die statistische Absicherung 
untermauerte die beobachteten Unterschiede zwischen den Erntezeitpunkten, jedoch nicht den Unterschied zwischen den Baumarten (Tabelle 3.3.2).

Tabelle 3.3.2: P-Werte von ${ }^{13} \mathrm{C}$ - sowie ${ }^{15} \mathrm{~N}$ - Konzentration in Rinde, Feinwurzeln (FW) und Wurzelspitzen (WS) von Buche und Esche; Treatment: ${ }^{13} \mathrm{C}$ - und ${ }^{15} \mathrm{~N}$-Markierung;Kompartiment: Rinde, Feinwurzel, Wurzelspitzen; Faktoren: 1. Baumart 2. Erntetag

\begin{tabular}{|c|c|c|c|c|}
\hline Treatment & Kompartiment & $\mathrm{P}$ (Baumart) & $\mathrm{P}$ (Zeitpunkt) & $\mathrm{P}$ (Interaktion) \\
\hline${ }^{13} \mathrm{C}$ & Rinde & 0,166 & $<0,01$ & $<0,01$ \\
\hline${ }^{13} \mathrm{C}$ & Feinwurzel & 0,051 & 0,094 & 0,762 \\
\hline${ }^{13} \mathrm{C}$ & Wurzelspitzen & 0,01 & 0,119 & 0,285 \\
\hline${ }^{15} \mathrm{~N}$ & Rinde & $<0,01$ & $<0,01$ & $<0,01$ \\
\hline${ }^{15} \mathrm{~N}$ & Feinwurzel & 0,018 & 0,020 & 0,093 \\
\hline${ }^{15} \mathrm{~N}$ & Wurzelspitze & 0,035 & 0,024 & 0,213 \\
\hline
\end{tabular}

Der Kohlenstoff wurde durch die Rinde in die Feinwurzeln transportiert. Dies war an

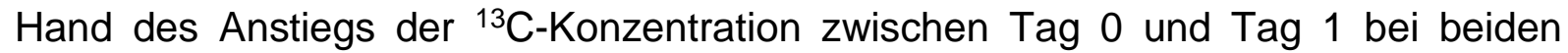
Baumarten bereits deutlich zu erkennen (Abbildung 3.3.1). Die natürliche Signatur $\left(\partial^{13} \mathrm{C}\right)$ der Feinwurzeln unterschied sich - wie bei der Rinde- zwischen beiden Baumarten nicht. Die Buche zeigte eine natürlich ${ }^{13} \mathrm{C}$-Signatur in der Feinwurzel von $-27,8$, die sich nicht merklich von der der Esche $\left(\partial^{13} \mathrm{C}=-27,7\right)$ unterscheidet. Die natürliche Signatur in der Rinde lag bei beiden Baumarten niedriger als in den Wurzeln.

Bereits 24 Stunden nach der Applikation von ${ }^{13} \mathrm{C}$ war in den Feinwurzeln der beiden Baumarten ein Anstieg an markiertem ${ }^{13} \mathrm{C}$ messbar. Im weiteren Verlauf zeigte die Esche eine etwas höhere Akkumulation von ${ }^{13} \mathrm{C}$ in der Feinwurzel als die Buche. Die statistische Untersuchung sicherte diese Beobachtung ebenfalls ab (Tabelle 3.3.1).

Die beiden Baumarten unterschieden sich in der Akkumulation von ${ }^{13} \mathrm{C}$ in den Feinwurzeln, wobei der Unterschied in den Wurzelspitzen noch deutlich stärker war. Die natürliche Signatur von ${ }^{13} \mathrm{C}$ in den Wurzelspitzen war in der Kontrolle ( Tag 0) mit $-28,9 \pm 0,7 \%$ bei Buche und $-32,6 \pm 0,2 \%$ bei Esche noch relativ ähnlich, unterschied sich aber in der Markierung zwischen den beiden Baumarten deutlich. Über den Verlauf der 60 Tage war die Akkumulation in der Buche im Vergleich zur Esche in den Wurzelspitzen signifikant geringer (Tabelle 3.3.2, Abbildung 3.3.1c). In den Eschenwurzelspitzen war ein starker Anstieg bis auf $342 \%$ an Tag $5 \mathrm{zu}$ erkennen. Ein hohes Niveau wurde relativ konstant über die weitere Versuchsdauer

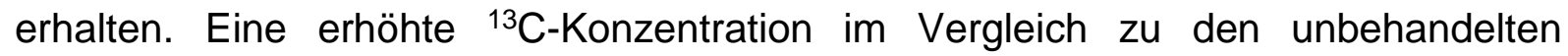
Bäumen wurde in den Buchenwurzelspitzen früher, bereits einen Tag nach der 
Markierung, erreicht und verblieb dann relativ konstant. Im Vergleich zur Esche verblieb die Konzentration in den Buchenwurzelspitzen jedoch insgesamt deutlich geringer. Bei beiden Baumarten war jedoch deutlich zu erkennen, dass die Akkumulation von ${ }^{13} \mathrm{C}$ in den Wurzelspitzen höher als in den Feinwurzeln war (Abbildung 3.3.1 b \& c). Im Mittel über die Markierungsphase betrug der ${ }^{13} \mathrm{C}$ Konzentrationsunterschied zwischen Wurzelspitzen und Feinwurzel bei der Buche 35 $\pm 17 \%$ und bei der Esche $171 \pm 97 \%$. An Tag 5, 20 und 60 betrug die Differenz zwischen Feinwurzeln und Wurzelspitzen bei der Esche sogar über $300 \%$. 

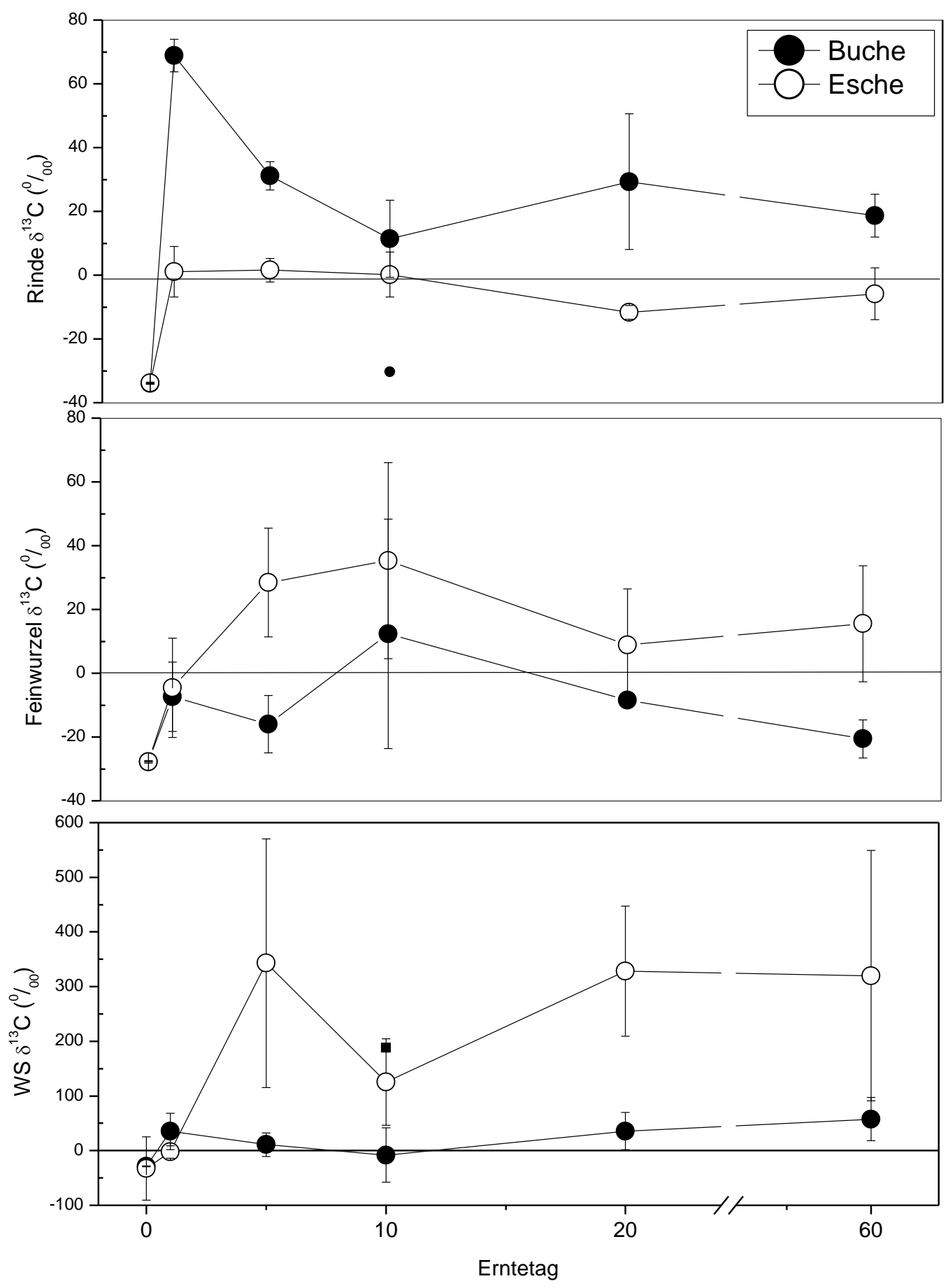

Abbildung 3.3.1: $\delta^{13} \mathrm{C}$ in Rinde (a), Feinwurzeln (b) und vitale Wurzelspitzen (c) von Buche und Esche über einen Zeitraum von 60 Tagen nach Begasung mit ${ }^{13} \mathrm{CO}_{2}$ für 5 Stunden (WS = Wurzelspitze). Die Bäume wurden an Tag 0 (Kontrolle) sowie 1, 5, 10, 20 und 60 (markierte Bäume) nach der Kohlenstoffmarkierung beprobt. Die Daten zeigen Mittelwerte \pm SE, $n=4-5$ (Tag 0 (Kontrolle) bzw. $n=$ 3- 4 pro Tag). 
3.3.1.2. Stickstoffmarkierung in Rinde, Feinwurzel und vitalen Wurzelspitzen von Buche und Esche

Die natürliche Signatur (atom $\%{ }^{15} \mathrm{~N}$ ) in der Rinde war in der Buche 0,364998 \pm 0,0005 atom\% und in der Esche 0,36442 \pm 0,0006 atom\%, während sie in den Feinwurzeln bei der Buche bei 0,36541 \pm 0,0005 und der Esche bei 0,36484 \pm 0,0001 lag. Die natürliche Signatur in den Wurzelspitzen betrug in der Buche 0,36598 \pm 0,0005 und in der Esche 0,36450 \pm 0,0003 atom\%. Die natürlichen Signaturen der beiden Baumarten waren in den Kompartimenten Feinwurzel ( $p=$ $0,029)$ und Wurzelspitzen $(p<0,01)$, in der Rinde $(p=0,143)$ jedoch nicht signifikant unterschiedlich. Für die markierten Proben wurde ${ }^{15} \mathrm{~N}$ atom\%excess berechnet. ${ }^{15} \mathrm{~N}$ in der Rinde der Buche akkumulierte über die Zeit stetig, jedoch mit geringer Steigung (Abbildung 3.3.2 a) im Vergleich zur Esche. Die Anreicherung von ${ }^{15} \mathrm{~N}$ in der Eschenrinde stieg konstant bis Tag 20 (0,41 \pm 0,21 APE (atom\%excess)) an und fiel dann geringfügig ab (0,32 $\pm 0,20$ APE). Der Unterschied zwischen den beiden Baumarten in der Akkumulation von ${ }^{15} \mathrm{~N}$ in der Rinde war signifikant (Tabelle 3.3.1). 


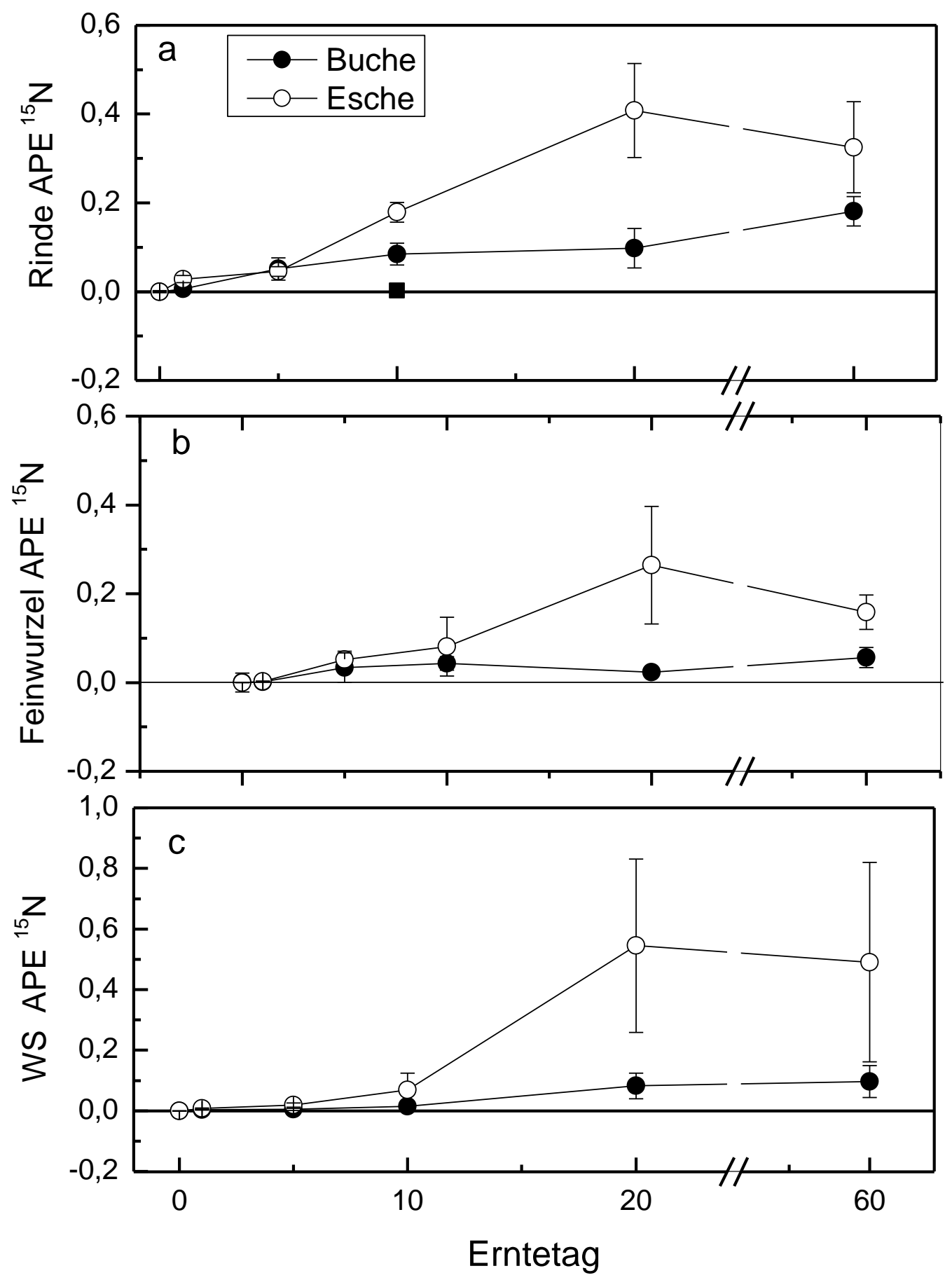

Abbildung 3.3.2: ${ }^{15} \mathrm{~N}$ in Rinde (a), Feinwurzel (b) und vitale Wurzelspitzen (c) von Buche und Esche über einen Zeitraum von 60 Tagen nach Begasung mit ${ }^{13} \mathrm{C}$ für 5 Stunden (WS = Wurzelspitze).Die Bäume wurden an Tag 0 (Kontrolle) sowie 1, 5, 10, 20 und 60 (markierte Bäume) nach der Kohlenstoffmarkierung beprobt. Die Daten zeigen Mittelwerte \pm SE, $n=4-5$ (Tag 0 (Kontrolle) bzw. $n=$ 3- 4 pro Tag. APE = atom\%excess

Ein deutlicher Unterschied in der Akkumulation von ${ }^{15} \mathrm{~N}$ zeigte sich auch in den Feinwurzeln. Hier war zu erkennen, dass bei der Buche die Akkumulation im 
Vergleich zur Esche - wie bei der Baumrinde - geringer war (Abbildung 3.3.2 b). Die Konzentration in der Buche war mit durchschnittlich 0,0017 \pm 0,0560 APE im Zeitraum Tag 1- 60 nicht höher als in der Kontrolle (Abbildung 3.3.2 c). Im Vergleich beider Baumarten zeigte sich eine höhere Konzentration von ${ }^{15} \mathrm{~N}$ in den Eschenfeinwurzeln (Abbildung 3.3.1 b). Das APE -Maximum wurde dabei an Tag 20 mit 0,26 \pm 0,13 APE erreicht und fiel zum Ende des Beobachtungszeitraums leicht ab. Der Unterschied in der ${ }^{15} \mathrm{~N}$-Akkumulation zwischen beiden Baumarten war ebenfalls in den Wurzelspitzen zu erkennen (Tabelle 3.3.2). Während die Akkumulation über die Zeit in der Buche - wie auch in der Feinwurzel - relativ gering blieb, war bei der Esche ab Tag 10 ein deutlicher Anstieg zu erkennen, der an Tag 20 das Maximum $(0,54 \pm 0,57$ APE) erreichte und dann relativ konstant blieb (Abbildung 3.3.2 c).

Tabelle 3.3.3: P-Werte ${ }^{13} \mathrm{C}$ - sowie ${ }^{15} \mathrm{~N}$ - Konzentration in Buchen und Eschen; Kompartiment: Rinde, Feinwurzel, vitale Wurzelspitzen; Faktoren: 1. Kompartiment 2. Zeitpunkt

\begin{tabular}{|c|c|c|c|c|}
\hline Treatment & Baumart & $\mathrm{P}$ (Kompartiment) & $\mathrm{P}$ (Zeitpunkt) & $\mathrm{P}$ (Interaktion) \\
\hline${ }^{13} \mathrm{C}$ & Buche & 0,029 & 0,172 & 0,797 \\
\hline${ }^{13} \mathrm{C}$ & Esche & $<0,01$ & 0,090 & 0,212 \\
\hline${ }^{15} \mathrm{~N}$ & Buche & 0,02 & $<0,01$ & 0,210 \\
\hline${ }^{15} \mathrm{~N}$ & Esche & 0,32 & $<0,01$ & 0,826 \\
\hline
\end{tabular}

Der Vergleich der einzelnen Gewebekompartimente innerhalb der Baumarten zeigte, dass sich die Akkumulation von ${ }^{13} \mathrm{C}$ in beiden Baumarten in den einzelnen Gewebekompartimenten unterschied, jedoch unabhängig von den einzelnen Untersuchungszeitpunkten (Tabelle 3.3.3). Es zeigte sich weiter, dass sich die Akkumulation von $\mathrm{APE}^{15} \mathrm{~N}$ in der Esche zwischen Rinde, Feinwurzeln und Wurzelspitze nicht signifikant unterschied; dies war jedoch in der Buche der Fall (Tabelle 3.3.1). Dort zeigte sich ein signifikanter Unterschied zwischen der der Rinde und den Feinwurzeln.

Um zu prüfen, ob es einen Zusammenhang im Transport von $\mathrm{C}$ und $\mathrm{N}$ gibt, wurden Korrelationen durchgeführt. Die Korrelationen von ${ }^{13} \mathrm{C}$ und ${ }^{15} \mathrm{~N}$ in den drei Kompartimenten Rinde, Feinwurzeln und Wurzelspitzen zeigten jedoch weder bei der Buche (Abbildung 3.3.3) noch bei der Esche (Abbildung 3.3.4) einen Zusammenhang. Diese Analyse ist ohnehin problematisch, auf Grund der zeitlichen Unterschiede in der Applikation von $\mathrm{C}$ und $\mathrm{N}$. 


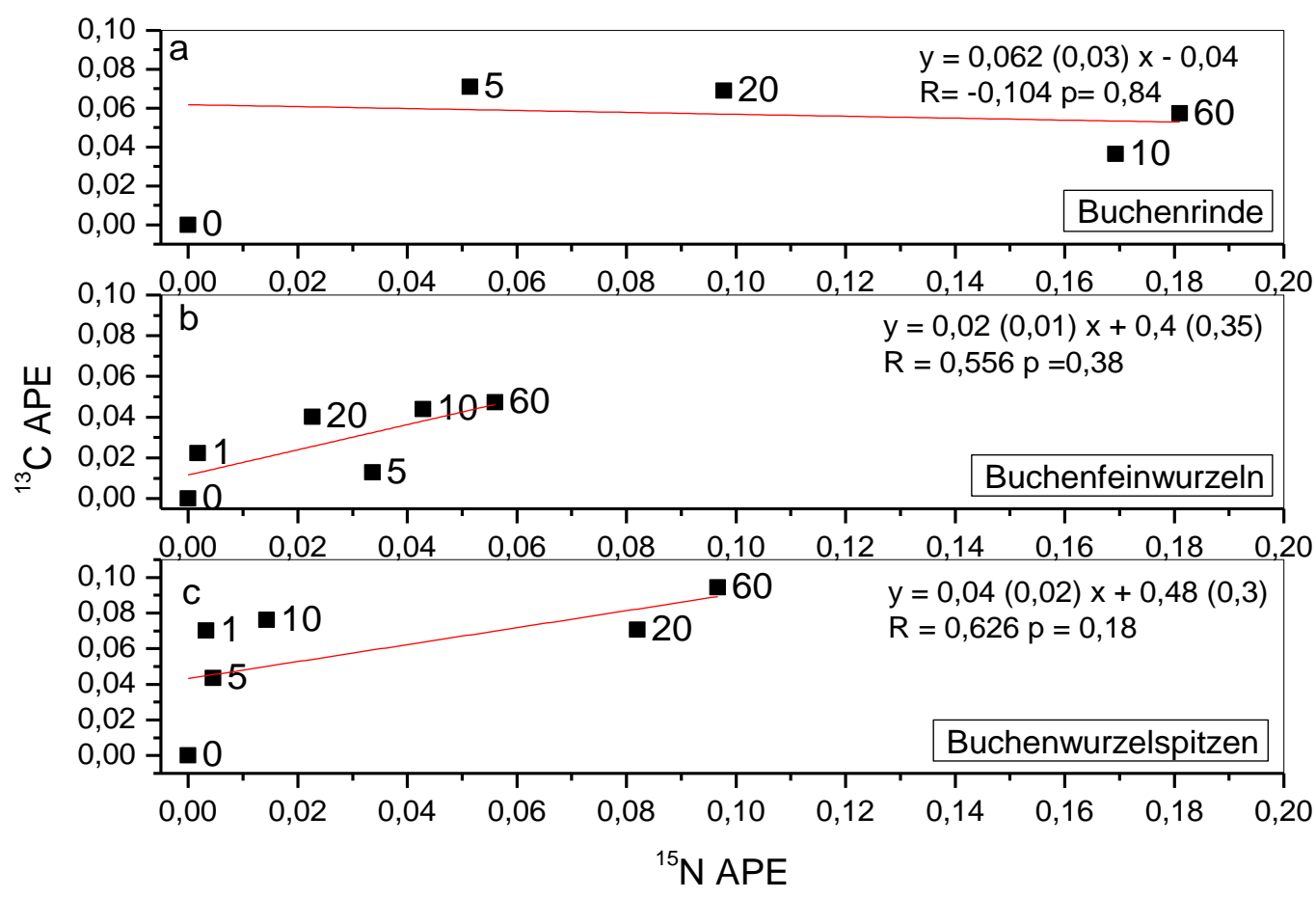

Abbildung 3.3.3: Korrelation von ${ }^{13} \mathrm{C}$ und ${ }^{15} \mathrm{~N}$ in Buchenrinde (a), Buchenfeinwurzeln (b) und Buchenwurzelspitzen (c). Die Daten zeigen Mittelwerte \pm SE, $n=4-5$ (Tag 0 (Kontrolle) bzw. $n=3-4$ pro Tag.APE $=$ atom $\%$ excess 


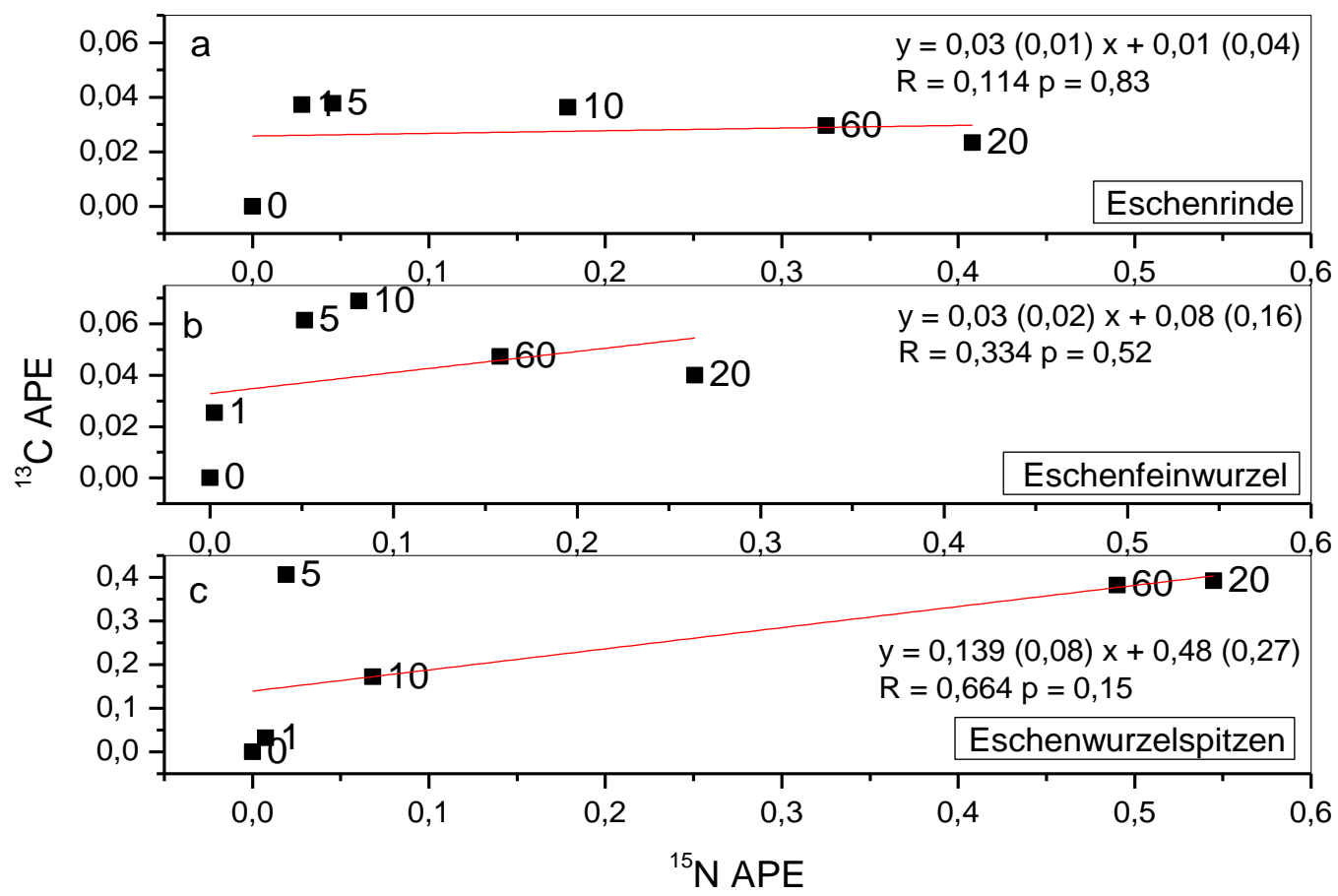

Abbildung 3.3.4: Korrelation von ${ }^{13} \mathrm{C}$ und ${ }^{15} \mathrm{~N}$ in Eschenrinde (a), Eschenfeinwurzeln (b) und Eschenwurzelspitzen (c). Die Daten zeigen Mittelwerte \pm SE, $n=4-5$ (Tag 0 (Kontrolle) bzw. $n=3-4$ pro Tag. APE = atom\%excess 


\section{4 Diskussion}

In der vorliegenden Freilanduntersuchung wurden Unterschiede in der Kinetik des Stofftransportes von ${ }^{13} \mathrm{C}$ und ${ }^{15} \mathrm{~N}$ in Buchen und Eschen zu ihrem Wurzelsystem bis in die Wurzelspitzen aufgezeigt. Die beiden Baumarten, die mit unterschiedlichen Mykorrhizaformen vergesellschaftet sind (Smith and Read, 2008), zeigten zudem verschieden hohe Stoffakkumulationen in den einzelnen Geweben Rinde, Feinwurzel und Wurzelspitzen. Unterschiede in der Wurzelmasse, die auch Meinen et al. (2009) gefunden haben, bestätigten sich auch in dieser Untersuchung, weil die Feinwurzeltrockenbiomasse der Buche nahezu doppelt so groß wie die der Esche ist.

Nach der Begasung der Bäume konnten wir den ${ }^{13} \mathrm{C}$-Transport an Hand der Akkumulation in der Rinde bei der Esche an Tag 5 und bei der Buche bereits an Tag 1 nachweisen. Bei der postexperimentellen Betrachtung ergibt sich die Schlussfolgerung, dass ein früherer Messzeitpunkt in Bezug auf die Buchen sinnvoll gewesen wäre, da der Transport von Photosyntheseprodukten durch das Phloem anscheinend sehr schnell beginnt. Diese Schlussfolgerung wird durch die Beobachtung von Keel et al. (2007) unterstützt, in deren Freilandbegasung die Markierung binnen weniger Stunden in der Rinde von Altbäumen detektierbar war. Dies deckt sich auch mit der Beobachtung von Högberg et al. (2008), wonach die Markierung vier Stunden nach Beendigung einer ${ }^{13} \mathrm{CO}_{2}-\mathrm{Begasung}_{\text {von }}$ jungen Kiefern messbar war.

Nichtsdestotrotz akkumulierten Buchen und Eschen unterschiedliche Konzentrationen an ${ }^{13} \mathrm{C}$ in der Rinde. Dies wurde auch in einem Gewächshausversuch mit markierter Laubstreu festgestellt (Cesarz et al., 2013), wobei wir in unserer Untersuchung jedoch nur einen tendenziellen Unterschied feststellen konnten.

Dass der Transport von Photosyntheseprodukten relativ kurzfristig verläuft zeigte auch die Akkumulation von ${ }^{13} \mathrm{C}$ in der Feinwurzel. Beide Baumarten akkumulierten bereits am ersten Tag nach der Markierung Kohlenstoff in ihren Feinwurzeln. Im Vergleich dazu, wurde die Akkumulation in Feinwurzeln in einem Freilandexperiment an Kiefern nach 4-7 Tagen beobachtet (Högberg et al., 2008). Die signifikant höhere Akkumulation der Eschenfeinwurzeln wird durch die Ergebnisse der obengenannten Studie von Cesarz et al. (2013) bestätigt. Daraus bilden sich die unterschiedlichen Strategien der Baumarten heraus. Während die Buche eine nicht unerhebliche 
Konzentration an ${ }^{13} \mathrm{C}$ in der Rinde akkumulierte, transportierte die Esche ${ }^{13} \mathrm{C}$ in größerem $\mathrm{Maß}$ in die Feinwurzel. Beiden Baumarten gemein ist die deutlich höhere Akkumulation von ${ }^{13} \mathrm{C}$ in den Wurzelspitzen im Vergleich zur Feinwurzel, was unsere einleitende Frage, welches der beiden Kompartimente der stärkere Sink für Kohlenstoff sei, beantwortet. Die größere Anreicherung von ${ }^{13} \mathrm{C}$ im mykorrhizanahen Wurzelbereich im Vergleich zur restlichen Feinwurzel wurde ebenfalls in einem Versuch mit jungen Buchen gezeigt (Valtanen et al., 2014). Die Sinkgröße des photosynthetischen Kohlenstoffs von ektomykorrhizierten Wurzelspitzen wurde bereits durch Högberg et al. (2008) aufgezeigt. Demgegenüber steht in unserer Untersuchung die signifikant höhere Akkumulation von ${ }^{13} \mathrm{C}$ in den Wurzelspitzen der Esche. Dies widerspricht den Beobachtungen von Eissfeller et al. (2013), wonach derer die Akkumulation von ${ }^{13} \mathrm{C}$ in der Mykorrhiza der Buche höher als bei der Esche war, nachdem die Pflanzen für fünf Monate einer erhöhten ${ }^{13} \mathrm{CO}_{2}$-Atmosphäre im Gewächshaus ausgesetzt waren.

Untersuchungen mit stabilem Stickstoff als Tracer erfolgen methodisch meist über die Zugabe über den Boden (Millard et al., 2005; Zein et al.,2011;Pena et al. ,2013).In unserer Untersuchung konnten wir zeigen, dass beide Baumarten Stickstoff auch dann in ihr Wurzelsystem transportieren, wenn dieser über die Blätter zugegeben wird. Ein kausaler Zusammenhang von Kohlenstoff und Stickstoff im pflanzeninternen Stofftransport konnten wir dabei nicht feststellen; jedoch unterschiedliche Transportstrategien von Buche und Esche beobachten. Während die Buche Stickstoff aus den Blättern lediglich durch Rinde, Feinwurzel und Wurzelspitzen „durchlaufen“ lässt, scheint die Esche ${ }^{15} \mathrm{~N}$ über die Zeit in den Kompartimenten $\mathrm{zu}$ speichern. Diese Beobachtung wirft unterschiedliche Interpretationen auf. Zum einen könnte die Buche den Stickstoff über Wurzelexsudate der Ektomykorrhiza bereits weiter in den Boden transportiert haben. Da dies bei der arbuskulär-mykorrhizierten Esche nicht der Fall ist, könnte diese den Stickstoff in ihrem eigenen Wurzelsystem einlagern. Dies würde auf einen Unterschied der beiden Baumarten auf Grund ihrer Mykorrhizaform hindeuten. Andererseits kann die höhere Konzentration von ${ }^{15} \mathrm{~N}$ in der Feinwurzel und den Wurzelspitzen auch durch die dickeren Feinwurzeln und den generell höheren Stickstoffgehalt der Feinwurzeln von Esche erklärt werden. Dies wird ebenfalls in 
einer Untersuchung in Hinblick auf das Konkurrenzverhalten der beiden Baumarten festgestellt (Beyer et al., 2013). Da der Einfluss der Mykorrhizaform in unserer Untersuchung nicht eindeutig bewiesen werden kann, scheint die Beobachtung von Beyer et al. (2013) auch für unsere Untersuchung schlüssig, nach der Eschenfeinwurzeln mehr $\mathrm{N}$ und höhere $\delta{ }^{13} \mathrm{C}$-Werte aufweisen als Buchenfeinwurzeln, was aus dem signifikanten Baumartenidentitätseffekt resultierte.

Aus unserer Untersuchung haben sich folgende Schlussfolgerungen ergeben: Bei beiden Baumarten stellen die Wurzelspitzen einen stärkeren Sink für Kohlenstoff dar als die Feinwurzeln; die Baumarten zeigen allerdings Unterschiede in der Höhe der Kohlenstoffakkumulation und -transportgeschwindigkeit. Unterschiede zwischen den beiden Baumarten treten ebenfalls in Bezug auf den Stickstofftransport auf, wobei die Gründe nicht durch unsere Untersuchung aufzudecken sind. Es konnte allerdings gezeigt werden, dass der Transport beider Nährelemente in keinem Zusammenhang steht. Ob es einen Unterschied in der Transportgeschwindigkeit zwischen Kohlenstoff und Stickstoff gibt, konnte auf Grund der zeitlich versetzten Applikation der beiden Stoffe nicht untersucht werden. 


\subsection{LITERATUR}

Abuzinadah RA, Read DJ (1986a) The role of proteins in the nitrogen nutrition of ectomycorrhizal plant. II Utilization of protein by mycorrhizal plants of Pinus contorta. New Phytol 103:495-506

Abuzinadah RA, Read DJ (1986a) The role of proteins in the nitrogen nutrition of ectomycorrhizal plant. III. Protein utilisation by Betula, Picea and Pinus with Hebeloma crustuliniforme. New Phytol 103:495-506

Beyer F, Hertel D, Leuschner C (2013) Fine root morphological traits in Fagus sylvatica and Fraxinus excelsior sapling as dependent on species, root order and competition. Plant Soil 373:143-156

Broadmeadow M, Ray D (2005) Climate change and British woodland. Forest Commission Information 69

Brumme R, Leimke U, Matzner E (1992) Interception and uptake of $\mathrm{NH}_{4+}$ and $\mathrm{NO}_{3}-$ from wet deposition by above-ground parts of young beech (Fagus sylvatica). Plant Soil 142: 273-279

Cesarz S, Fender AC, Beyer F, Valtanen K, Pfeiffer B, Gansert D, Hertel D, Polle A, Daniel R,Leuschner C,Scheu S(2013) Roots from beech (Fagus sylvatica L.) and ash (Fraxinus excelsior L.) differentially affect soil microorganisms and carbon dynamics. Soil Biol Biochem 61: 23-32

Ellenberg H (1996)Vegetation Mitteleuropas mit den Alpen. Ulmer Verlag Stuttgart 5. Auflage

Eissfeller V, Beyer F, Valtanen K, Hertel D, Maraun M, Polle A, Scheu S (2013) Incorporation of plant carbon and microbial nitrogen into the rhizosphaere food web of beech and ash. Soil Biol Biochem 62: 76-81

Grime JP, Thompson K, Hunt R, Hodgson JG, Cornelissen JHC, Rorison IH, Hendry GAF, Ashenden TW, Askew AP, Band SR, Booth RE, Bossard CC, Campbell BD, Cooper JEL, Davison AW, Gupta PL, Hall W, Hand DW, Hannah MA, Hillier SH, Hodkinson DJ, Jalili A, Liu Z, Mackey JML, Matthews N, Mowforth MA, Neal AM, Reader RJ, Reiling K, Ross-Fraser W, Spencer RE, Sutton F, Tasker DE, Thorpe PC, Whitehouse J (1997) Integrated creening validates primary axes of specialization in plants. Oikos 79:259-281

Geßler A, Schultze M, Schrempp S, Rennenberg H (1998) Interaction of phloemtranslocated amino compounds with nitrate net uptake by the roots of beech (Fagus sylvatica). J Exp Bot 49: 1529-1537

Geßler A, Weber P, Schneider S, Rennenberg H (2003) Bidirectional exchange of amino compounds beween phloem and xylem during long-distance transport in Norway spruce trees (Picea abies (Karst) L.). J Exp Bot 54: 1389-1397

He X, Xu M, Qui GY, Zhou J (2009) Use of $15 \mathrm{~N}$ stable isotope to quantify nitrogen transfer between mycorrhizal plants. J Plant Ecol 2:107-118

Högberg P, Högberg MN, Gottlicher SG, Betson NR, Keel SG, DB Metcalfe, Campbell C, Schindlbacher A, Hury V, Lundmark T, Linder S, Näshom T (2007) High 
temporal resolution tracing of photosynthate carbon from the tree canopy to forest soil organisms. New Phytol 177: 220-228

Keel S, Siegwolf RTW, Jäggi M, Körner C(2007) Rapid mixing between old and new $\mathrm{C}$ pools in the canopy of mature forest trees. Plant Cell Environ 30: 963- 972

Keel S, Campbell CD, Högberg MN, Richter A, Wild B, Zhou X, Hurry V, Linder S, Näsholm T, Högberg P (2012) Allocation of carbon to fine root compounds and their residence times in a boreal forest depend on root size class and season. New Phytol 194:972-981

Marschner H, Dell B (1993)Nutrient uptake in mycorrhizal symbiosis. Plant Soil 159:89-102

Meinen C, Hertel D, Leuschner C (2009) Biomass and morphology of fine roots in temperate broad-leaved forests differing in tree species diversity. Is there evidence of below-ground overyielding? Oecologia 161:99-111

Millard P, Wendler R, Grassi G, Grellet GA, Tagliavini M (2005) Translocation of nitrogen in the xylem of field-crown cherry and poplar trees during remobilization. Tree Physiol 26: 527- 536

Näsholm T, Kielland K, Ganeteg U (2009) Uptake of organic nitrogen in plants. New Phytol 182:31-48

Pena R, Tejedor J, Zeller B, Dannenmann M, Polle A (2013) Interspecific temporal and spatial differences in the acquisition of litter-derived nitrogen by ectomycorrhizal fungal assemblages. New Phytol 199:520-528

Philip LJ, SW Simard SW (2008) Plant Soil Vol. 308 "Minimum pulses of stable and radioactive carbon isotopes to detect belowground carbon transfer between plants. Plant Soil 308: 23-35

Read DJ, Perez- Moreno J (2003) Mycorrhizas and nutrient cycling in ecosystems - a journey towards relevance? New Phytol 157: 475-492

Smith S, Read DJ (2008) Mycorrhizal symbiosis. Acad Press, Cambridge, UK

Schulz H, Härtling S,Stange CF (2011) Species-specific differences in nitrogen uptake and utilization by six European tree species. J. Plant Nutr Soil Sci174:28-37

Valtanen K, Beyer F, Hertel D,Scheu S, Polle A (2014) Carbon fluxes between beech and their ectomycorrhizal assemblage. Mycorrhiza. short note

Wilson EJ (1992) Foliar uptake and release of inorganic nitrogen compounds in Pinus sylvestris L. and Picea abies (L.) Karst.. New Phytol 120:407-416

Zein K, Breda B, Gerant D, Zeller Z, Maillard P (2011) Nitrogen sources for currentyear shoot growth in 50 year-old sessile oak trees: an in situ ${ }^{15} \mathrm{~N}$ labeling approach. Tree Physiol 31:1390-1400 
4. Kapitel: Vitalitätsuntersuchungen an Wurzelspitzen von Buche (Fagus sylvatica) und Esche (Fraxinus excelsior)

\subsection{Einleitung}

Baumarten unterscheiden sich in der Biomasse ihrer Feinwurzeln sowie der Verteilung der Feinwurzeln im Bodenraum (Büttner \& Leuschner,1994). Der Unterschied zeigte sich auch in Freilanduntersuchungen (Schmid \& Kazda,2002), wobei beobachtet wurde, dass die Biomasse von Feinwurzeln in Reinbeständen geringer als in Mischbeständen war (Cueva et al. ,1991; Schmid \& Kazda,2002).In diesem Zusammenhang zeigten Untersuchungen in Beständen unterschiedlichen Baumartenreichtums, dass sich die Biomasse der toten Wurzeln, die Nekromasse, jedoch nicht unterscheidet (Meinen et al.,2009). In diesen Untersuchungen zeigte die Hauptbaumart Buche eine tendenzielle Erhöhung der Feinwurzelbiomasse mit zunehmendem Baumartenreichtum der Bestände. Die häufig in Verbindung mit der Buche vorkommende Esche (Fraxinus excelsior) zeigte in einer anderen Untersuchung eine höhere Feinwurzelproduktion in Beständen mittleren Baumartenreichtums (Meinen et al., 2009b). Mit zunehmendem Artenreichtum nahm dabei auch die Nekromasse der Feinwurzeln der Esche zu (Meinen et al., 2009 b). In Wurzeluntersuchungen ist die Abschätzung des Vitalitätsgrades der untersuchten Feinwurzeln schwierig. In der vorliegenden Untersuchung wollen wir dazu lebende und tote Wurzelspitzen von Buche und Esche daher an Hand morphologischer und anatomischer Eigenschaften charakterisieren und überprüfen, ob Wurzeln, die als tot angesprochen wurden auch wirklich abgestorben sind. Dazu sollen die Wurzeln zum einen visuell angesprochen werden und die Anatomie von lebenden Wurzelquerschnitten mit als tot angesprochenen Wurzelquerschnitten auf etwaige Unterschiede verglichen werden. Da dabei davon ausgegangen wird, dass als tot angesprochene Wurzeln auch physiologisch inaktiv sind, soll der Ansatz an Hand von TTC-Tests und dem Einsatz von stabilen Isotopen als Tracer in einem Transportexperiment überprüft werden.

Es wird geschätzt, dass bis zu $1 / 3$ des Kohlenstoffes aus der jährlichen globalen Nettoprimärproduktion in den unterirdischen Feinwurzelbereich investiert wird (Jackson et al.,1997). Die Feinwurzelbiomasse kann im Vergleich von Baumarten bis zu 53\% variieren (Finér et al.,2011). 
Bezogen auf unsere einheimischen Wälder zeigte sich in einer Freilanduntersuchung in Buchenbeständen verschiedener Niederschlagsmengen und Bodenversauerung in Deutschland, dass sich die Feinwurzelbiomasse nicht, die Feinwurzelnekromasse jedoch schon zwischen den Standorten verschiedener $\mathrm{pH}-$ Werte unterschied (Leuschner et al.,2004).

Die Lebensdauer von Wurzeln von Buchen beträgt rund 10 Monate (Brunner et al. ,2011). Die Umsatzrate von Wurzeln hat einen mittelbaren Einfluss auf die Kohlenstoffallokation im Boden (Pregitzer et al., 1995). Durch eine Erhöhung des atmosphärischen Kohlendioxidgehaltes wird der turnover von Wurzeln beschleunigt. In einem FACE (free air enrichment) - Experiment wurde in diesem Zusammenhang an Hand von TTC (Triphenyltetrazoliumchlorid)- Tests festgestellt, dass Wurzeln, die unter Pinus-Arten wuchsen, eine 55\% höhere Dehydrogenase-Enzymaktivität unter erhöhter $\mathrm{CO}_{2}$-Konzentration zeigten als Wurzeln, die unter Larix-Arten wuchsen (Handa et al. ,2008). TTC ist ein typischer Vitalitätsmarker zur Unterscheidung zwischen lebenden und toten Zellen in Bäumen (Joslin \& Henderson, 1984), da TTC lediglich durch vitale Zellen reduziert werden kann. Der TTC-Test wird unter anderem zur Vitalitätsbestimmung von Pflanzenkeimlingen verwendet (Lassheikki et al.,1991)). Die Vitalitätsbestimmung von Wurzeln kann neben der Verwendung von TTC auch visuell erfolgen, wonach die verschiedenen Stadien lebend bzw. vital und trocken bzw. tot optisch bestimmt werden (Clemensson-Lindell, 1994). Tests haben gezeigt, dass die visuelle Betrachtung und Einschätzung von Wurzeln mit Hilfe von Kamera und Minirhizotron möglich ist und eine Genauigkeit von $85 \%$ der Methode der Färbung mit TTC erreichen kann (Wang et al.,1995).

Im Zusammenhang mit TTC- Tests wurden zur Vitalitätsbestimmung von Wurzeln auch anatomische Querschnitte zur Analyse von Zellveränderungen verwendet (Comas et al., 2000).

In der vorliegenden Untersuchung soll dies angewandt werden und in Kombination mit TTC-Tests beurteilt werden, ob die visuelle Einteilung von Wurzelspitzen in "vital" (d.h. lebend) und "trocken" (d.h. tot) bei den einheimischen Baumarten Buche und Esche anwendbar ist. Die Methode soll zudem an Hand von Untersuchungen zum Stofftransport überprüft werden und die Hypothese bestätigen, dass tote Wurzeln von einer Stoffakkumulation ausgeschlossen sind. Die Grundlage für die vorliegende Untersuchung sind Freilanduntersuchungen, in denen ${ }^{13} \mathrm{C}$ über die Photosynthese in 
vitalen Wurzeln akkumuliert wurden (Eissfeller et al.,2013; Endrulat et al.,2010; Winkler et al.,2010). Wir nutzen diesen Effekt, um zu überprüfen, ob die trocken aussehenden Wurzeln auch tatsächlich kein ${ }^{13} \mathrm{C}$ aufnehmen, um zu untermauern, dass visuelle Ansprachen von Wurzeln zur Klassifizierung nach lebend und tot möglich sind. Die Untersuchung der Aufnahme von $\mathrm{N}$ und $\mathrm{C}$ in trockenen Wurzelspitzen ist die neuerliche Grundidee dieses Experimentes, da davon ausgegangen werden kann, dass tote Wurzeln physiologisch inaktiv sind und demnach keine Stoffakkumulation erfolgt. Diese Tatsache soll in der vorliegenden Untersuchung überprüft werden. 


\subsection{Material \& Methoden}

Für die Vitalitätsuntersuchungen wurden im Untersuchungsgebiet "Lichte Haardt" $\left(51^{\circ} 05^{\prime} 28^{\prime \prime} \mathrm{N}, 10^{\circ} 31^{\prime} 24^{\prime \prime}\right.$ E) (siehe Kapitel 3) ein Markierungsexperiment durchgeführt und für weitere Untersuchungen in der Billingshäuser Schlucht $\left(51^{\circ} 52^{\prime} 33^{\prime \prime} \mathrm{N} \mathrm{12}{ }^{\circ} 11^{\prime} 22^{\prime \prime}\right.$ E) unter jungen Buchen und Eschen mit Hilfe eines Bohrkerns (4,7 cm Durchmesser, 20cm Länge) Bodenkerne entnommen. Die Bäume in der Billingshäuser Schlucht entsprachen von der Höhe und des Standortes denen, die im Markierungsexperiment (siehe Kapitel 3) verwendet wurden und auf die in der vorliegenden Untersuchung Bezug genommen wird. Die Proben in der Billingshäuser Schlucht wurden für die TTC-Untersuchungen vor Ort genommen, um möglichst frische Wurzeln zu untersuchen. Für die optische Ansprache und die anatomischen Querschnitte wurden, neben den Proben aus dem Markierungsexperiment, Proben in der „Lichten Haardt" entnommen, bei $4^{\circ}$ Celsius im Kühlraum aufbewahrt und innerhalb einer Woche verarbeitet. Die Analyse der für die TTC-Tests verwendeten Wurzeln erfolgte unmittelbar nach der Probennahme im Bestand.

\subsubsection{Optische Ansprache der Wurzelspitzen}

Die Wurzeln wurden aus den Bohrkernen entnommen, unter Leitungswasser vorsichtig gesäubert und bis zur Analyse bei $4^{\circ}$ Celsius gelagert. Die optische Ansprache der Wurzel erfolgte unter einer Stereolupe (M 205 FA, Leica, Wetzlar, Deutschland). Dazu wurden die Wurzeln in eine, mit Wasser gefüllte, Petrischale gegeben. Die optische Ansprache zur Unterscheidung zwischen lebenden und toten Wurzeln erfolgte an Hand der Farbe und Oberflächenbeschaffenheit. Lebende Wurzeln zeigen eine glatte Oberflächenstruktur. Trockene Wurzeln sehen durch den Wasserverlust an ihrer Oberfläche „verschrumpelt“ aus. Vitale und trockene Wurzelspitzenproben wurden mit Hilfe einer Pinzette in, mit angefeuchtetem Filterpapier ausgelegten, Petrischalen gesammelt und unmittelbar zur Erstellung anatomischer Querschnitte eingebettet (siehe 4.2.2). 


\subsubsection{Einbettung der Wurzelspitzen zur Erstellung anatomischer Querschnitte}

Vitale und trockene Wurzelspitzen von Buche und Esche wurden mit StyrolMethacrylat fixiert und eingebettet. Dazu wurden vitale und trockene Wurzelspitzen abgesammelt und in einem Eppendorf-Reaktionsgefäß (Eppendorf, Hamburg, Deutschland) in FAE (Formaldehyd- Ethanol) für drei Tage gelagert. Anschließend wurden die Wurzeln wie folgend beschrieben über einen Ethanol/Aceton-Gradienten entwässert und anschließend in Plastik eingebettet. Dazu wurden Ethanol und Aceton auf einem Molekularsieb (Sigma-Aldrich, Hannover, Deutschland) vorbereitet und dreimal ausgetauscht. Die Wurzelspitzen wurden zu Beginn für 2 Stunden in 80 \%-igen Ethanol inkubiert, der danach durch 90\%-igen Ethanol für weitere zwei Stunden ersetzt wurde, worauf eine weitere zweistündige Inkubation mit 100\% Ethanol erfolgte. Die Inkubation über die verschiedenen Ethanol/Aceton/PlastikStufen erfolgte unter ständiger Bewegung. Nach der zweistündigen Entwässerung mit 100\%-igem Ethanol wurde dieser ausgetauscht und die Wurzelspitzen inkubierten bei Raumtemperatur über Nacht. Die Inkubation über Nacht wurde beendet, indem der 100\%-ige Ethanol in den Reaktionsgefäßen durch ein Gemisch aus 50\%-igem Ethanol und 50\%-igem Aceton (1:1) ersetzt wurde und die Wurzelspitzen darin für zwei Stunden weiterhin inkubiert wurden. Anschließend erfolgte die Inkubation für zwei Stunden in 100\%-igem Aceton. Um jegliche Rückstände des Ethanols zu entfernen, wurde das Aceton ausgetauscht und die Proben wiederum für 1,5 Stunden inkubiert. Darauf folgte eine schrittweise Umsetzung der Wurzelspitzen in eine flüssige Plastikmischung aus $15 \mathrm{ml}$ Styrene (Merck-Schuchardt, Darmstadt, Deutschland), $15 \mathrm{ml}$ Butyl-Methacrylat (SigmaAldrich, Seelze, Deutschland) und 0,3 g Dibenzoylperoxid (BP-50-FT-Peroxid Chemie GmbH, Pullach, Deutschland). Für die schrittweise Umsetzung der Wurzelspitzen wurden diese nach der Acetoninkubation in ein Gemisch aus 100\%igem Aceton und der obigen Plastikmischung (Verhältnis 1:1) gegeben und für 4 Stunden bei Raumtemperatur inkubiert. Danach wurde dieses Gemisch durch eines aus den oben genannten Komponenten ersetzt (Aceton:Plastik-Verhältnis 1:3) und die Wurzelspitzen darin über Nacht inkubiert. Am vierten Tag wurden die Wurzelspitzen in die Plastikmischung umgesetzt. Nach der ersten Inkubationsphase für 4,5 Stunden wurde die Plastikmischung ausgetauscht und erneut über Nacht inkubiert. Nach der Inkubation über Nacht wurde das reine Plastik erneuert. Nach einer viereinhalbstündigen Inkubation wurde der Vorgang wiederholt und die 
Wurzelspitzen erneut über Nacht in reinem Plastik belassen. Am darauffolgenden Morgen wurden die Wurzelspitzen zur Einbettung in Gelatinekapseln $\left(0,36 \mathrm{~cm}^{3}\right)$ umgesetzt. Dazu wurden die Gelatinekapseln in Reaktionsgefäßständer gegen Umkippen fixiert und mit ca. $300 \mu \mathrm{l}$ des gleichen Plastiks, in dem die Wurzelspitzen auch über Nacht inkubiert wurden, gefült. In die Gelatinekapseln wurde dann jeweils eine Wurzelspitze gegeben, mit Plastik aufgefüllt und mit dem Kapseldeckel geschlossen. Die Wurzelspitzen in den Gelatinekapseln wurden durch vorsichtiges Drehen der Kapsel in Senkrechtlage gebracht; die Luftblasen in der Kapsel lagerten sich dabei am oberen Rand an, während die Wurzelspitzen an den unteren Rand der Kapsel fielen. Zur Aushärtung des Plastiks zur Vorbereitung der Querschnitte wurden die Kapseln leicht angekippt in Aluschälchen für einen Tag bei $60^{\circ} \mathrm{Celsius} \mathrm{im}$ Trockenschrank (Memmert GmbH \& CoKG, Schwabach, Deutschland) gelagert. Dies diente dem Starten des Aushärtungsprozesses. Danach wurden die Kapseln für die vollständige Aushärtung für zehn Tage bei $37^{\circ}$ Celsius im Trockenschrank verwahrt.

Nach der Aushärtung wurden von den vitalen und trockenen Wurzelspitzen von Buche und Esche wurden $1 \mu \mathrm{m}$ dünne Querschnitte angefertigt (Ultracut E Mikrotom, Reichert- Jung, Depew, USA). Die Querschnitte wurden mit Toluidinblau (0,5\% Toulidinblau-Zinkchlorid (Certistain, Merck, Darmstadt, Deutschland) in 1\% Borsäure (Roth, Karlsruhe, Deutschland) angefärbt, in Depex-Eindeckmittel fixiert (Serva, Heidelberg, Deutschland) und mit Hilfe eines Mikrokops (Axioplan, Zeiss, Jena) in 200- und 400facher Vergrößerung fotografiert.

\subsubsection{Vitalitätsbestimmung der Wurzelspitzen an Hand von TTC-Tests}

Die optische Ansprache der Wurzelspitzen in Bezug auf Vitalität wurde mit Hilfe eines TTC (2,3,5-triophenyltetrazoliumchlorid)-Tests überprüft. Dieser Test wird zum Nachweis intakter Zellen genutzt. Grundlage bei diesem Test nach Altman (1976) ist eine Redoxreaktion der Tetrazoliumverbindung TTC, die sich bei Reaktion mit lebendem Zellgewebe in einem Farbumschlag äußert. Dabei handelt es sich um das Ergebnis der Reduktion des Tetrazolium-Kations durch Dehydrogenasen der Atmungskette zu Formazan (Ogur et al., 1957; Rich et al.,2001). Das TTC fungiert im Test als Indikator und ist im oxidierten Zustand (Tetrazolium) farblos; färbt sich im reduzierten Zustand (Formazan) jedoch rot. Für die Vitalitätsbestimmung wurden von, frisch aus dem Boden entnommenen Wurzeln, jeweils Wurzelspitzen vitaler und trockener Erscheinung mit einer Pinzette abgezupft und für 24 Stunden bei leichtem 
Schütteln im Dunklen bei $25^{\circ}$ Celsius in $1 \mathrm{ml}$ TTC-Lösung inkubiert. Die TTC-Lösung wurde durch die Zugabe von $1 \mathrm{mg}$ TTC (Merck, Darmstadt, Deutschland) in $100 \mathrm{ml}$ 0,05 M Phosphat-Puffer hergestellt. Der Phosphatpuffer wurde durch Zugabe von 3,4 $\mathrm{mg} \mathrm{KH}_{2} \mathrm{PO}_{4}$ in $500 \mathrm{ml}$ destilliertes Wasser vorbereitet und auf einen $\mathrm{pH}$-Wert von 5,8 eingestellt. Nach der 24-stündigen Inkubation wurden die Proben dreimal mit destilliertem Wasser gewaschen. Nach der Zugabe von jeweils 1,5 ml Ethanol auf die Proben wurden diese $30 \mathrm{~min}$ bei $70^{\circ} \mathrm{C}$ elsius im Thermoblock temperiert. Dadurch wurde der aufgenommene Indikator in die Lösung freigesetzt. Nach folgendem Abkühlen in Eiswasser wurden die Proben gemischt und für $15 \mathrm{~min}$ bei $15.000 \mathrm{rpm}$ bei $4^{\circ} \mathrm{C}$ zentrifugiert (Centrifuge 5417R, Eppendorf, Hamburg, Deutschland).Vom Überstand wurden $700 \mu \mathrm{l}$ abgenommen und in Kunststoffküvetten bei $485 \mathrm{~nm}$ im Photometer (Beckman Coulter $\mathrm{GmbH}$, Krefeld, Deutschland) gemessen. Vitale Wurzelspitzen zeigen bei dieser Wellenlänge eine Absorption des reduzierten Farbstoffes. Zur Kontrolle wurde 99\%-iger Ethanol bei gleicher Wellenlänge als „Blank“ gemessen. Der Vitalitätsindex wurde in Extinktion/mg angegeben. Die Prozedur wurde an Wurzelspitzen, die in einem Trockenschrank (Memmert, Schwabach, Deutschland) für 1 Woche bei $60^{\circ} \mathrm{Celsius}$ getrocknet wurden, wiederholt.

\subsubsection{Analyse der ${ }^{13} \mathrm{C}$ - und ${ }^{15} \mathrm{~N}$-Akkumulation in lebenden und toten Wurzelspitzen}

Die Akkumulation von ${ }^{13} \mathrm{C}$ und ${ }^{15} \mathrm{~N}$ in den lebenden und toten Wurzelspitzen von Buche und Esche wurde an Hand eines Markierungsexperimentes durchgeführt. Diese Untersuchung erfolgte unter Freilandbedingungen im Nationalpark Hainich (siehe Kapitel 3). Die Bäume wurden dazu über die Blätter für 3 Tage mit einer ${ }^{15} \mathrm{~N}$ markierten Calciumnitrat $\left(\mathrm{Ca}\left({ }^{15} \mathrm{NO}_{3}\right)\right)$ - Lösung inkubiert und anschließend für 5 Stunden mit $99 \%$-igem ${ }^{13} \mathrm{CO}_{2}$ begast. Eine detaillierte Versuchsbeschreibung enthält Kapitel 3.2 dieser Arbeit. Die Analyse der Isotopensignatur der Wurzelspitzen wurde an Hand Massenspektrometrie durchgeführt und ist in Kapitel 3.2.5 dieser Arbeit detailliert beschrieben.

\subsubsection{Statistik}

Die statistische Auswertung erfolgte mit Microsoft Excel (Windows, Redmond, USA) sowie Origin Pro 8.5 (OriginLab Corporation, Northampton, MA, USA). Die Daten wurden an Hand von Shapiro-Wilk beziehungsweise Kolmogorov-Smirnov Test auf 
Normalverteilung getestet. Die Daten wurden an Hand Zwei-Wege-ANOVA (Origin Pro 8.5,OriginLab Corporation, Northampton, MA, USA mit den Faktoren „Erntetag“ bzw. „Vitalität“ und „Baumart" analysiert.

\section{3 Ergebnisse}

4.3.1 Analyse der morphologischen Merkmale an Hand von Oberflächenaufnahmen und anatomischen Querschnitte

Um die Unterschiede in den morphologischen Merkmalen zu analysieren, wurden die lebenden und trockenen Wurzelspitzen von Buche und Esche fotografiert (Abbildung 4.3.1 a-d). Es zeigte sich dabei, dass die vitalen Wurzelspitzen von Buche eine hellbraune, glatte Oberfläche aufwiesen (Abbildung 4.3.1 a), wohingegen die trockenen Wurzelspitzen deutlich dunkler waren und ihre Oberfläche durch den Wasserverlust "verschrumpelt" aussahen (Abbildung 4.3.1 b). Im Vergleich zur Buche zeigten sich die vitalen Wurzelspitzen bei der Esche ebenfalls glatt, waren aber von ihrer Oberflächenerscheinung heller, sogar leicht durchsichtig (vgl. Abbildung 4.3.1 a \& 4.3.1 c). Die trockenen Wurzelspitzen der Eschen waren in der Farbe deutlich dunkler und sahen - wie die der Buchen- "verschrumpelt" aus (Abbildung 4.3.1. d).

a)

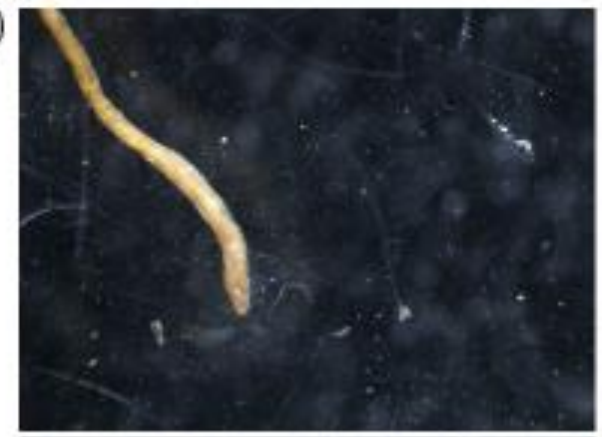

c)

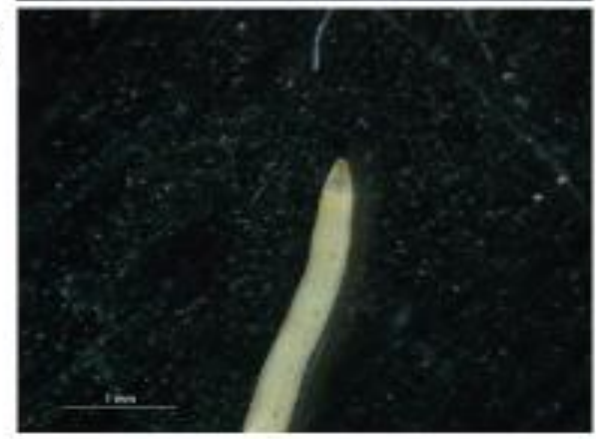

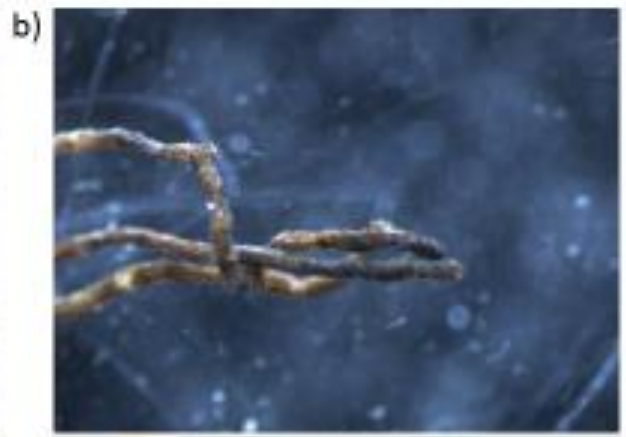

d)

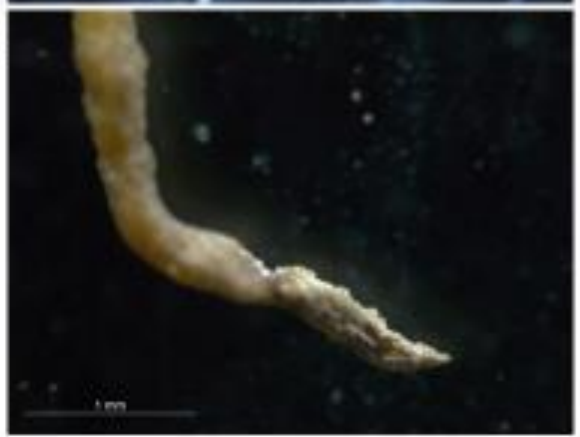

Abbildung 4.3.1: Fotografien von vitaler (a) und trockener (b) Wurzelspitze von Buche sowie von vitaler (c) und trockener (d) Wurzelspitze von Esche 
Ein deutlicher Unterschied zwischen vitalen und trockenen Wurzelspitzen war zum Teil ebenfalls in den angefertigten anatomischen Querschnitten erkennbar (Abbildung 4.3.2 a-d). In der vitalen Wurzelspitze der Buche waren die einzelnen Zellen in ihrer ovalen Form gut im Zellverband erkennbar (Abbildung 4.3.2 a). Durch den Wasserverlust schrumpften die Zellen zusammen und der Zellverband wurde teilweise aufgelöst (Abbildung 4.3.2 b). Dagegen waren bei den Eschen zwischen vitalen (Abbildung 4.3.2 c) und trockenen (Abbildung 4.3.2 d) Wurzelspitzen kaum Unterschiede zu erkennen.

a)

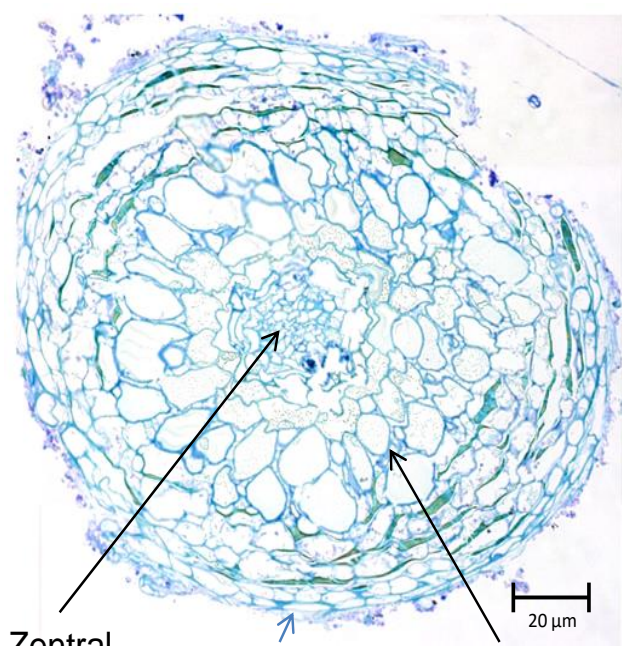

c) Zen zyir
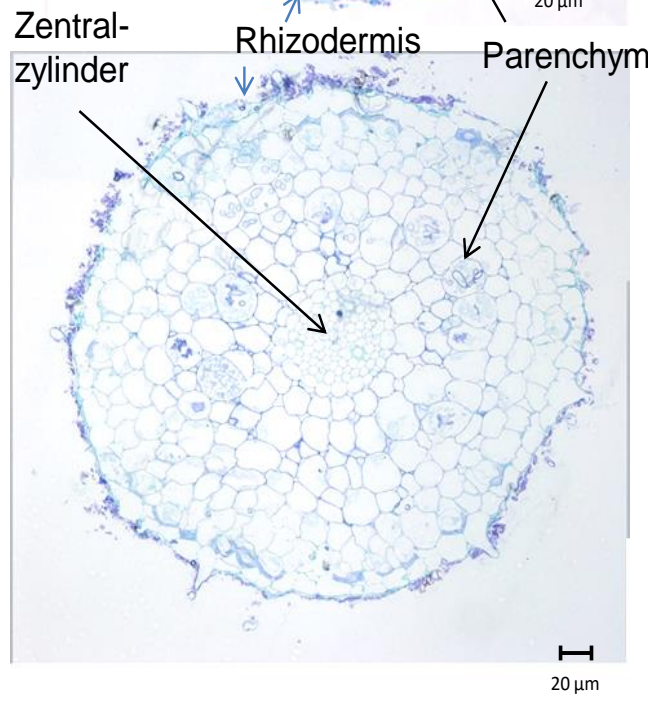

b)

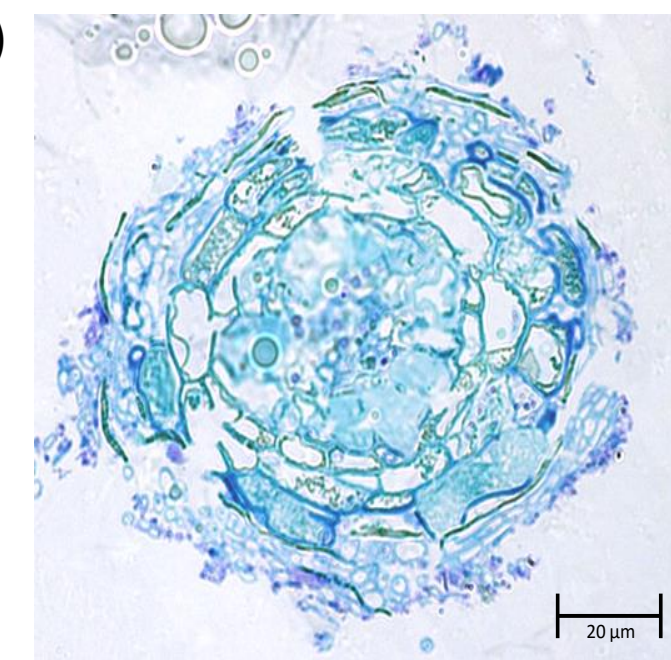

d)

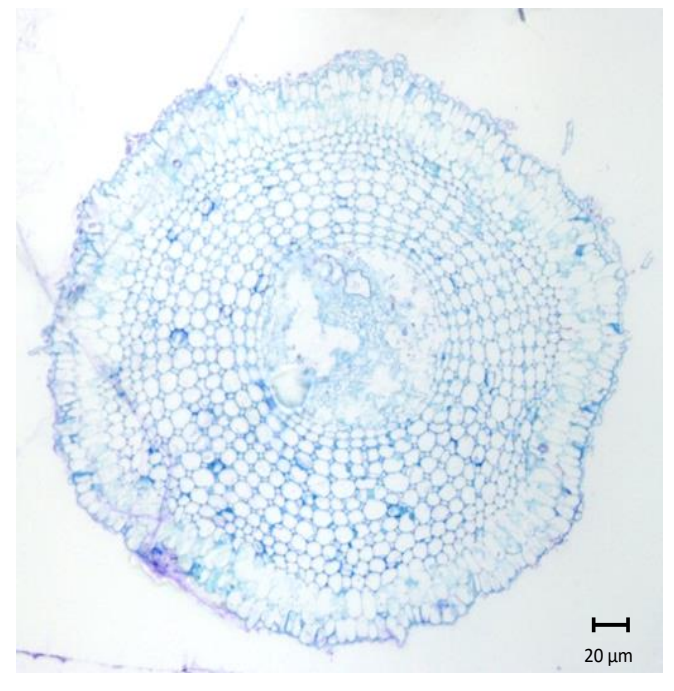

Abbildung 4.3.2: Querschnitte von vitaler (a) und trockener (b) Wurzelspitze von Buche sowie von vitaler (c) und trockener (d) Wurzelspitze von Esche 


\subsubsection{TTC-Tests an vitalen und trockenen Wurzelspitzen}

Um zu überprüfen, ob die als trocken angesprochenen Wurzelspitzen physiologisch inaktiv waren, wurden TTC-Tests durchgeführt. Die Wurzelspitzen der beiden Baumarten unterschieden sich dabei in der Extinktion der Lichtabsorption. Die vitalen Wurzelspitzen der Esche zeigen mit einer durchschnittlichen Extinktion von 4,18 \pm 2,30 units TTC $_{\text {red/ }} g$ eine signifikant höhere Absorption des reduzierten Farbstoffes ( $p$ $=0,02)$ als die der Buche, deren vitale Wurzelspitzen eine durchschnittliche Extinktion von 2,39 $\pm 1,31$ units TTC red/ $g$ aufwiesen (Abbildung 4.3.3). Die trockenen Wurzelspitzen der Buche zeigten nur eine sehr geringe Absorption reduzierten Farbstoffes $\left(0,83 \pm 1,11\right.$ units TTC $\left._{\text {red/ }} \mathrm{g}\right)$, während die trockenen Wurzelspitzen der Esche mit 2,08 \pm 1,66 units TTC $_{\text {red/g) }}$ ) nur gering unter der der vitalen Wurzelspitzen der Buche lagen (Abbildung 4.3.3). Eine Absorption reduzierten Farbstoffes in den als "trocken" angesprochenen Wurzelspitzen der Esche war demnach messbar.

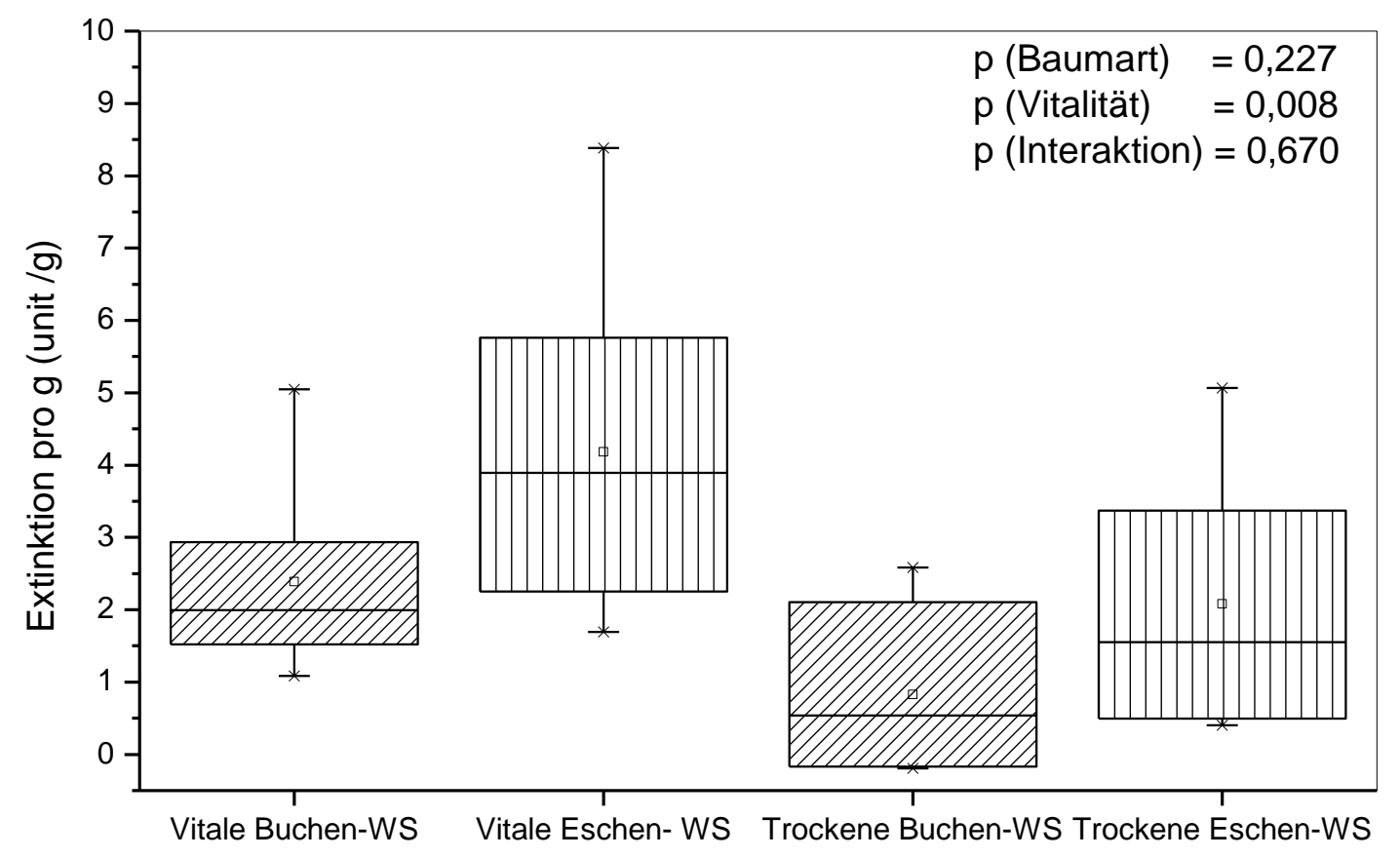

Abbildung 4.3.3: Extinktion von TTC (TTC red /g) in den Wurzelspitzen von Buche und Esche, $\mathrm{n}=7$ (pro Wurzelspitzentyp und Baumart) (red= reduziert)

In der Analyse der visuellen Ansprache und den TTC-Tests zeigte sich, dass beide Methoden bei der Buche anwendbar sind, wohingegen bei der Esche optisch kein 
deutlicher Unterschied zwischen vitalen und trockenen Wurzelspitzen erkennbar war und die Methode demnach schwierig erschien. Hinzu kam eine Absorption in den als trocken angesprochenen Eschenwurzelspitzen. Um dies zu überprüfen, wurde ein Transportexperiment, das im Rahmen des Markierungsexperimentes im Nationalpark Hainich (siehe Kapitel 3) durchgeführt wurde, analysiert.

\subsection{3 ${ }^{13} \mathrm{C}$ - und ${ }^{15} \mathrm{~N}$ - Transport in trockene Wurzelspitzen}

Nach der Markierung mit ${ }^{13} \mathrm{C}$ und ${ }^{15} \mathrm{~N}$ wurden trockene Wurzelspitzen von Buche und Esche untersucht. In den trockenen Wurzelspitzen der Buchen konnte keine Akkumulation von $\partial{ }^{13} \mathrm{C}$ festgestellt werden. Die Signatur der trockenen Wurzelspitzen der Buche lag bei $0 \pm 0,0009 \mathrm{APE}^{13} \mathrm{C}$ (atom\%excess ${ }^{13} \mathrm{C}$ ) für die Kontrollen und zwischen $0,0049 \pm 0,00095 \mathrm{APE}^{13} \mathrm{C}$ und $0,00867 \pm 0,007 \mathrm{APE}^{13} \mathrm{C}$ nach Markierung ( $p=0,21$ )(Abbildung 4.3.4 A). Die Kontrollen beider Baumarten unterschieden sich nicht, aber die markierten trockenen Wurzelspitzen der Esche zeigten im Vergleich zur markierten Buche einen signifikanten Unterschied $(p<$ 0,01 ). Bei der Esche wurde in den markierten Wurzelspitzen zwischen 0,036 $\pm 0,02$ $\mathrm{APE}{ }^{13} \mathrm{C}$ und $0,094 \pm 0,051 \mathrm{APE}{ }^{13} \mathrm{C}$ gemessen (Abbildung 4.3.4 A).

Eine Akkumulation in den trockenen Wurzelspitzen der Esche zeigte sich ebenfalls bezüglich des Stickstoffs; in den trockenen Wurzelspitzen der Buche konnte jedoch keine erhöhte Konzentration von ${ }^{15} \mathrm{~N}$ gemessen werden. In den trockenen Wurzelspitzen der Esche zeigte sich die höchste Konzentration - wie beim Kohlenstoff- am Ende der Versuchsdauer (Abbildung 4.3.4 B). Die maximale Konzentration wurde mit $0,54 \pm 0,65$ atom\%excess ${ }^{15} \mathrm{~N}\left(\mathrm{APE}^{15} \mathrm{~N}\right.$ ) an Tag 20 gemessen und nahm zum Ende des Versuchszeitraums wiederum leicht ab $(0,37 \pm$ $0,57 \mathrm{APE}^{15} \mathrm{~N}$ an Tag 60). 


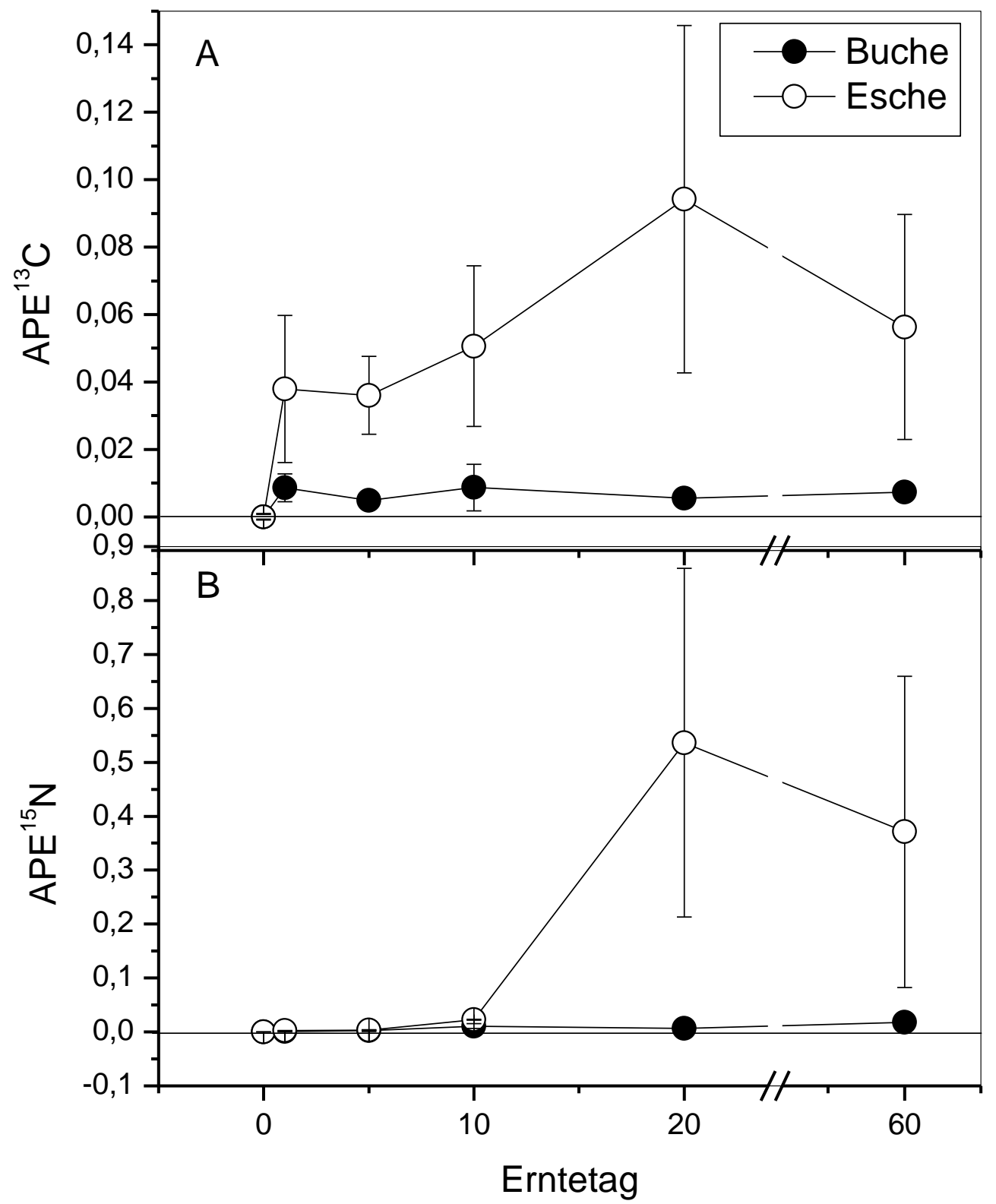

Abbildung 4.3.4: Kohlenstoffkonzentration (A) und Stickstoffkonzentration (B) in den trockenen Wurzelspitzen von Buche und Esche über den Versuchszeitraum von 60 Tagen (MW $\pm S E, n=5$ (Tag 0 (Kontrolle) bzw. $\mathrm{n}=4$ pro Tag (Tag 1,5,10,20,60(markiert)) 
Der Vergleich der Atom\%excess-Werte, bezogen auf ${ }^{13} \mathrm{C}$ und ${ }^{15} \mathrm{~N}$, zeigte, dass die gemessenen Werte in den trockenen Wurzelspitzen, mit Ausnahme des 20. Versuchstages bei der Esche (bezogen auf ${ }^{15} \mathrm{~N}$ ) geringer als in den vitalen Wurzelspitzen war (Tabelle 4.3.1).

Tabelle 4.3.1: Atom\%excess ${ }^{13} \mathrm{C}(\mathrm{A})$ und Atom\%excess ${ }^{15} \mathrm{~N}(\mathrm{~B})$ in vitalen (MWS) und trockenen (TWS) Wurzelspitzen in Buche und Esche über den Versuchszeitraum von 60 Tagen (MW \pm SE, $n=5$ (Tag 0 (Kontrolle) bzw. $\mathrm{n}=4$ pro Tag (Tag 1,5,10,20,60(markiert))

\begin{tabular}{|c|c|c|c|c|}
\hline \multirow[t]{3}{*}{ A } & \multicolumn{4}{|c|}{ Atom\%excess ${ }^{13} \mathrm{C}$} \\
\hline & \multicolumn{4}{|c|}{ Buche } \\
\hline & \multicolumn{2}{|l|}{ MWS } & \multicolumn{2}{|l|}{ TWS } \\
\hline Tag & Mittelwert & SE & Mittelwert & SE \\
\hline 0 & 0,0000000 & 0,0007155 & 0 & 0,0009334 \\
\hline 1 & 0,0700679 & 0,0365137 & 0,0085782 & 0,0041124 \\
\hline 5 & 0,0435669 & 0,0236172 & 0,0049012 & 0,000951 \\
\hline 10 & 0,0762717 & 0,0540813 & 0,0086692 & 0,0069611 \\
\hline 20 & 0,0706437 & 0,0372264 & 0,0054715 & 0,001051 \\
\hline \multirow[t]{2}{*}{60} & 0,0944669 & 0,0431053 & 0,0073487 & 0,0019021 \\
\hline & \multicolumn{4}{|l|}{ Esche } \\
\hline \multirow[b]{2}{*}{ Tag } & \multicolumn{2}{|l|}{ MWS } & \multicolumn{2}{|l|}{ TWS } \\
\hline & Mittelwert & SE & Mittelwert & SE \\
\hline 0 & $1,332 \mathrm{E}-16$ & 0,0007291 & $-4,44 \mathrm{E}-17$ & 0,0007266 \\
\hline 1 & 0,0323673 & 0,0125007 & 0,0378829 & 0,0218325 \\
\hline 5 & 0,4064633 & 0,2459495 & 0,0360324 & 0,0115177 \\
\hline 10 & 0,1717798 & 0,086548 & 0,0506084 & 0,0238358 \\
\hline 20 & 0,3920073 & 0,1288288 & 0,0942537 & 0,0515489 \\
\hline 60 & 0,3815303 & 0,2477541 & 0,0562889 & 0,0333632 \\
\hline \multirow[t]{2}{*}{ B } & \multicolumn{4}{|c|}{ Atom\%excess ${ }^{15} \mathrm{~N}$} \\
\hline & \multicolumn{4}{|c|}{ Buche } \\
\hline \multirow[b]{2}{*}{ Tag } & \multicolumn{2}{|l|}{ MWS } & \multicolumn{2}{|l|}{ TWS } \\
\hline & Mittelwert & SE & Mittelwert & SE \\
\hline 0 & 0,0000000 & 0,0002181 & $-1,11 \mathrm{E}-17$ & 0,0001689 \\
\hline 1 & 0,0032547 & 0,0012514 & 0,0005508 & 0,0002572 \\
\hline 5 & 0,0045250 & 0,0033940 & 0,0024872 & 0,0015066 \\
\hline 10 & 0,0142898 & 0,0068806 & 0,0109109 & 0,004584 \\
\hline 20 & 0,0820055 & 0,0418179 & 0,0068312 & 0,0032244 \\
\hline \multirow[t]{2}{*}{60} & 0,0966227 & 0,0522477 & 0,0182061 & 0,0040062 \\
\hline & \multicolumn{4}{|l|}{ Esche } \\
\hline Tag & \multicolumn{2}{|l|}{ MWS } & \multicolumn{2}{|l|}{ TWS } \\
\hline 0 & $-2,22 \mathrm{E}-17$ & 0,0001274 & 4,441E-17 & $9,406 \mathrm{E}-05$ \\
\hline 1 & 0,0075729 & 0,0022633 & 0,0024546 & 0,000309 \\
\hline 5 & 0,0190516 & 0,0072545 & 0,0030135 & 0,000587 \\
\hline 10 & 0,0682327 & 0,0553649 & 0,0225453 & 0,0093243 \\
\hline 20 & 0,544881 & 0,285907 & 0,5363771 & 0,3233965 \\
\hline 60 & 0,4903109 & 0,3291228 & 0,3710799 & 0,2883953 \\
\hline
\end{tabular}


Das Verhältnis der ${ }^{13} \mathrm{C}$-Akkumulation von trockenen Wurzelspitzen zu vitalen Wurzelspitzen war in den Kontrollen der Buche mit 1,245 $\pm 1,5$ APE ${ }^{13} \mathrm{C}$ annähernd dreimal so hoch wie in denen der Esche, die in den Kontrollen ein ${ }^{13} \mathrm{C}$ - Verhältnis trocken/vital von 1,095 $\pm 0,09 \mathrm{APE}^{13} \mathrm{C}$ aufwiesen (Tabelle 4.3.2). Der Unterschied in den Kontrollen war in den beiden Baumarten jedoch nicht signifikant $(p=0,30)$. Das trocken/vital-Verhältnis war in den markierten Wurzelspitzen geringer als in den Kontrollen (Tabelle 4.3.2), jedoch nicht signifikant geringer $(p=0,15)$. Das trocken/vitalVerhältnis betrug in den markierten Buchen über den Versuchszeitraum zwischen $0,115 \pm 0,05 \mathrm{APE}{ }^{13} \mathrm{C}$ und $0,545 \pm 0,28 \mathrm{APE}{ }^{13} \mathrm{C}$ und in den Eschen zwischen 0,21 \pm 0,04 APE ${ }^{13} \mathrm{C}$ und $0,783 \pm 0,34$ APE ${ }^{13} \mathrm{C}$. Demnach war das Verhältnis in den Buchen etwas geringer als in den Eschen; ein signifikanter Unterschied in Bezug auf die Baumarten konnte dabei jedoch nicht festgestellt werden $(p=0,75)$.

Tabelle 4.3.2: ${ }^{13} \mathrm{C}$ APE-Verhältnis zwischen trockenen und vitalen Wurzelspitzen $\left({ }^{13} \mathrm{C}\right.$ APE TWS $/{ }^{13} \mathrm{C}$ APE MWS) in Buchen und Eschen an den Versuchstagen; Daten zeigen Mittelwert \pm SE

\begin{tabular}{|l|ll|ll|}
\hline Tag & $\begin{array}{l}\text { Buche } \\
\mathrm{APE}^{13} \mathrm{C}\end{array}$ & $\begin{array}{l}\text { APE } \\
\text { Mittelwert } \mathrm{C}\end{array}$ & $\mathrm{SE}$ & $\begin{array}{l}\text { Esche } \\
\mathrm{APE}^{13} \mathrm{C}\end{array}$ \\
& Mittelwert & APE ${ }^{13} \mathrm{C}$ \\
\hline 0 & 1,245 & 1,500 & 1,095 & 0,092 \\
1 & 0,351 & 0,219 & 0,783 & 0,340 \\
5 & 0,545 & 0,275 & 2,043 & 1,955 \\
10 & 0,115 & 0,051 & 0,432 & 0,116 \\
20 & 0,237 & 0,118 & 0,451 & 0,336 \\
60 & 0,141 & 0,075 & 0,210 & 0,040 \\
\hline
\end{tabular}

Das ${ }^{15} \mathrm{~N}$-Verhältnis von trockenen Wurzelspitzen zu vitalen Wurzelspitzen war in den Kontrollpflanzen in der Esche mit $-0,485 \pm 0,87$ APE ${ }^{15} \mathrm{~N}$ tendenziell etwas geringer $(p=0,49)$ als in der Buche, die ein trocken/vital-Verhältnis $0,230 \pm 0,44$ APE ${ }^{15} \mathrm{~N}$ aufwies (Tabelle 4.3.3). Mit Ausnahme des 20. Versuchstages, bezogen auf die Buche, wurden bei den markierten Bäumen größere trocken/vital-Verhältnisse im Vergleich zu den Kontrollen festgestellt. Dieser tendenzielle Unterschied war jedoch nicht signifikant $(p=0,57)$. Ausgenommen des 20 . Versuchstages wurden in der Buche zwischen $0,285 \pm 0,07$ APE ${ }^{15} \mathrm{~N}$ und 3,059 $\pm 2,98$ APE ${ }^{15} \mathrm{~N}$, mit abnehmender Tendenz über den Versuchszeitraum, beobachtet (Tabelle 4.3.3). Die Abnahme des Verhältnisses über die Zeit wurde bei der Esche nicht festgestellt. In den Wurzelspitzen der Esche wurden Verhältnisse zwischen 0,490 $\pm 0,19$ APE ${ }^{15} \mathrm{~N}$ und $2,101 \pm 1,79$ APE ${ }^{15} \mathrm{~N}$ festgestellt. Das Maximum wurden in den 
Eschenwurzelspitzen an Tag 20 beobachtet und nahm zum Ende des Versuches ab (Tabelle 4.3.3). Ein signifikanter Unterschied zwischen Buche und Esche wurde nicht festgestellt $(p=0,51)$.

Tabelle 4.3.3: ${ }^{15} \mathrm{~N}$ APE-Verhältnis zwischen trockenen und vitalen Wurzelspitzen $\left({ }^{15} \mathrm{~N}\right.$ APE TWS $/{ }^{15} \mathrm{~N}$ APE MWS) in Buchen und Eschen an den Versuchstagen; Daten zeigen Mittelwert \pm SE

\begin{tabular}{|c|c|c|c|c|}
\hline Tag & $\begin{array}{l}\text { Buche } \\
\text { APE }{ }^{15} \mathrm{~N} \\
\text { Mittelwert }\end{array}$ & $\begin{array}{l}\mathrm{APE}^{15} \mathrm{~N} \\
\mathrm{SE}\end{array}$ & $\begin{array}{l}\text { Esche } \\
\text { APE }^{15} \mathrm{~N} \\
\text { Mittelwert }\end{array}$ & $\begin{array}{l}\text { APE }{ }^{15} \mathrm{~N} \\
\text { SE }\end{array}$ \\
\hline 0 & 0,230 & 0,438 & $-0,485$ & 0,874 \\
\hline 1 & 3,059 & 2,978 & 0,490 & 0,189 \\
\hline 5 & 1,561 & 1,153 & 0,521 & 0,384 \\
\hline 10 & 1,388 & 0,487 & 0,920 & 0,486 \\
\hline 20 & 0,133 & 0,068 & 2,101 & 1,794 \\
\hline 60 & 0,285 & 0,069 & 0,582 & 0,088 \\
\hline
\end{tabular}




\subsection{Diskussion}

In der vorliegenden Untersuchung sollte überprüft werden, ob der methodische Ansatz der visuellen Ansprache von lebenden und toten Wurzelspitzen an Buche und Esche möglich ist. Die visuelle Ansprache von Wurzelspitzen wurde in Kombination mit Vitalitätsuntersuchungen auf der Grundlage von TTC bereits an Fichten angewendet und es zeigte sich dabei, dass die als lebend angesprochenen Wurzelspitzen eine höhere TTC-Aktivität zeigten als die toten Wurzelspitzen (Clemensson-Lindell, 1994). Wir haben die Methode auf zwei Laubbaumarten übertragen und es zeigte sich dabei, dass die Methode bei der Buche im Gegensatz zur Esche zuverlässig anwendbar ist. Bei der visuellen Analyse wurde festgestellt, dass zwischen den lebenden und toten Wurzelspitzen der Esche kaum ein anatomischer Unterschied wahrnehmbar war und beim TTC-Test trat eine Absorption des reduzierten Farbstoffes in den toten Eschenwurzeln auf. Die Methode sollte kritisch betrachtet werden, da bei der optischen Ansprache es immer möglich ist, dass einige als trocken angesprochenen Wurzelspitzen möglicherweise noch physiologisch aktiv sind. Dies konnten wir bei den Buchenwurzeln ausschließen, da sie sich zum einen anatomisch von den lebenden unterschieden und auch keine Farbstoffabsorption zeigten. Bei der Esche konnten wir durch die Absorption allerdings nicht ausschließen, dass die Wurzeln zwar in ihrer Aktivität eingeschränkt, jedoch nicht gänzlich tot waren. Um die die Aktivität der trockenen Eschenwurzeln weitergehend zu überprüfen, haben wir den Stofftransport von $\mathrm{C}$ und $\mathrm{N}$ in einem weiteren Experiment überprüft. Bei der Buche kam es über den Untersuchungszeitraum von 60 Tagen zu keiner nachweislichen Anreicherung von $\mathrm{C}$ und $\mathrm{N}$, wonach davon auszugehen ist, dass die Buchenwurzeln keinem nachweislichen turnover unterlagen und die Anwendbarkeit der Methode der visuellen Ansprache untermauert wird. Im Gegensatz dazu ließ die auftretende Akkumulation von $\mathrm{C}$ bei der Esche weiterhin eine Aktivität der trockenen Wurzeln vermuten, was das Ergebnis der visuellen Ansprache und der TTC-Tests untermauert, dass diese Methode für die Esche nicht anwendbar ist. Da die höchste Akkumulation dabei an Tag 5 beobachtet wurde, scheint es jedoch wahrscheinlich, dass die Eschenwurzeln zumindest einem turnover unterlagen und die Proben zumindest teilweise tot waren. Der Verlauf der Stickstoffanreicherung in den trockenen Eschenwurzeln war dem der lebenden ähnlich, sodass hier wiederum davon ausgegangen werden muss, dass die als tot angesprochenen Eschenwurzeln 
nicht vollständig abgestorben waren. Dies gibt wiederum den Beweis, dass die visuelle Ansprache bei der Esche nicht möglich ist.

Die vorliegende Untersuchung zeigte, dass die Methode der visuellen Ansprache nicht uneingeschränkt für verschiedene Baumarten anwendbar ist und auch der neuerliche Ansatz der Isotopenmarkierung dieses Ergebnis unterstreicht. 


\subsection{Literatur}

Brunner I, Bakker MR, Björk RG, Hirano Y, Lukac M, Arnada X, Borja I, Eldhuset TD, Helmissaari HS, Jourdan C, Konopka B, Lopez BC, Miguel Perez C, Persson H, Ostonen I (2012) Fine root turnover rates of European forests revisited: an analysis of data from sequential coring and ingrowth cores. Plant Soil 362: 357- 372

Büttner V, Leuschner C (1994) Spatial and temporal pattern of fine-root abundance in a mixed oak beech forest. For Ecol Manage 70:11-21

Clemensson- Lindell A (1994) Triphenyltetrazolium chloride as an indicator of fineroot vitality and environmental stress in coniferous forest stands: Applications and limitations. Plant Soil 159: 297- 300

Comas LH, Eissenstat DM, Lakso AN (2000) Assessing root death and root system dynamics in a study of grape canopy pruning. New Phytol 147: 171- 178

Cuevas E, Brown S, Lugo AE (1991) Aboveground and belowground organic-matter storage and production in a tropical pine plantation and a paired broadleaf secondary forest. Plant Soil 135: 257- 268

Eissfeller V, Beyer F, Valtanen K, Hertel D, Maraun M, Polle A, S. Scheu S(2013) Incorporation of plant carbon and microbial nitrogen into the rhizosphere food web of beech and ash. Soil Biol Biochem 62: $76-81$

Endrulat T, Sauer M, Buchmann N, Brunner I (2000) Incorporation and remobilization of ${ }^{13} \mathrm{C}$ within the fine- root system of individual Abies alba trees in a temperate coniferous stand. Tree Physiol. 30: 1515 - 1527

Finer L, Ohashi M, Noguchi K, Hirano Y (2011) Fine root production and turnover in forest ecosystems in relation to stand and environmental characteristics.

For Ecol Manag 262: 2008- 2023

Handa IT, Hagedorn F, Hättenschwiler S (2008) No stimulation in root production in response to 4 years of in situ enrichment in the Swiss treeline. Funct Ecol 22: 348358

Jackson RB, Mooney HA, Schulze ED (1997) A global budget for fine root biomass, surface area and nutrient content. Proc Nat Acad Sci USA 94: 7362- 7366

Joslin JD, Henderson GS (1984) The determination of percentages of living tissue in woody fine root samples using Triphenyltetrazolium chloride. For Sci 30: 965- 970 Lassheikki M, Puttonen P, Räsänen PK (1991) Planting performance potential of Pinus sylvestris seedlings as evaluated by root growth capacity and triphenyltetrazolium chloride reduction methods.Scand J For Res 6: 91- 104 
Leuschner C, Hertel D, Schmid I, Koch O, Muhs A, Hölscher D (2004) Stand fine biomass and fine root morphology in old-growth beech forests as a function of precipitation and soil fertility. Plant Soil 258: 43-56

Lukac M, Lagomarsino A, Moscatelle MC, de Angelis P, Cotrufo MF, Godbold DL (2008) Forest soil carbon cycle under elevated $\mathrm{CO}_{2}-$ a case of increased throughput?Forestry 82:75- 86

Meinen C, Hertel D, Leuschner C (2009a) Biomass and morphology of fine roots in temperate broad-leaved forests differing in tree species diversity: is there evidence of below-ground overyielding? Oecologia 161: 99- 111

Meinen C, Hertel D, Leuschner C (2009 b) Root growth and recovery in temperate broad-leaved forest stands differing in tree species diversity. Ecosys 12: 1103- 1116

Pregitzer KS, Zak DR, Kubiske ME, Teeri JA, Vogel CS (1995) Atmospheric $\mathrm{CO}_{2}$, soil nitrogen and turnover of fine roots. New Phytol 129:579-585

Schmid I, Kazda M (2002) Root distribution of Norway spruce in monospecific and mixed stands on different soils. For Ecol Manag 159:37- 47

Wang Z, Burch WH, P, Jones RJ, Mitchell RJ(1195) Accuracy of visible and ultraviolet light for estimating live proportions with minirhizotrons. Ecology 76: 2330 2334

Winkler JB, Dannenmann M, Simon J, Pena R, Offermann C, Sternad W, Clemenz C, Naumann PS, Gasche R, Kögel- Knabner I, Gessler A, Rennenberg H, Polle A (2010) Carbon and nitrogen balance in beech roots under competitive pressure of soil- borne microorganisms induced by girdling, drought and glucose application. Funct Plant Biol 37: 879- 889 
5. Kapitel: $\partial^{13} \mathrm{C}$ - und $\partial^{14} \mathrm{C}$ - Doppelmarkierung zur Analyse des Transportes von Kohlenstoff in die Rhizosphäre einheimischer Laubbaumarten

\subsection{Einleitung}

Die Ökosysteme unserer einheimischen Laubbaumwälder haben durch die Photosynthese und den damit verbundenen Kohlenstofftransport eine wichtige ökologische Funktion. Sie binden beispielsweise mehr als $92 \%$ des weltweiten terrestrischen Kohlenstoffs und speichern damit zwischen 20 und $100 \%$ mehr Kohlenstoff als Agrarflächen (Andrasko, 1990; Houghton, 1990). Auf Grund der CO $\mathrm{C}_{2}$ Erhöhung im Zuge der Globalerwärmung wird in den Wurzeln die Primärproduktion der Biomasse um 22 \% erhöht (Norby et al., 2004). Unsere einheimischen Laubbaumwälder bestehen aus verschiedenen Baumarten, wie zum Beispiel Ahorn (Acer spec.), Buche (Fagus sylvatica) und Linde (Tilia spec.). Diesen Baumarten gemein ist die Vergesellschaftung der Wurzeln mit Mykorrhizapilzen. Ein Kennzeichen dieser Symbiose ist der Transport von Kohlenstoff durch die Pflanze zum Pilz, der den Kohlenstoff zum Aufbau seiner Biomasse verwendet. Bis zu 30\% der Assimilationsprodukte werden zum Pilz transportiert, wobei die Pflanze im Gegenzug mit Nährstoffen wie zum Beispiel Stickstoff versorgt wird (Söderström \& Read, 1987). Die Symbiosen unterscheiden sich in Abhängigkeit von der Baumart. Buche und Linde bilden Ektomykorrhizen aus, während Ahorn mit Endomykorrhizen assoziiert ist.

Der Baumarten spezifische Kohlenstofftransport in Richtung der unterirdischen Wurzelsymbiose kann mit Hilfe von stabilen Isotopen untersucht werden. Kohlenstoff ist ein elementarer Nährstoff für die pflanzliche Biomasseproduktion und kommt zu ca $0,04 \%$ Bestandteil der Luft vor (Geilenkirchen, 1911). Der Kohlenstoff tritt darin natürlicherweise in verschiedenen Isotopen auf, wobei ${ }^{12} \mathrm{C}$ mit rund $98 \%$ dabei den größten Anteil einnimmt und das stabile Isotop ${ }^{13} \mathrm{C}$ mit $1,11 \%$ einen deutlich geringeren und das radioaktive Isotop ${ }^{14} \mathrm{C}$ mit $10^{-10} \%$ einen nahezu verschwindend geringen Anteil besitzt (Dyckmans 2013). Auf Grund der Menge und der CDiskriminierung in physiklaischen Prozessen, d.h. der Bevorzugung des leichteren Isotopes ${ }^{12} \mathrm{C}$ gegenüber den schwereren Isotopen, reichern Pflanzen bei der Photosynthese ${ }^{12} \mathrm{C}$ gegenüber ${ }^{13} \mathrm{C}$ in ihren Metaboliten an.

Der Transport und die Akkumulation von Kohlenstoff in Pflanzen, ihren Symbiosen und im angrenzenden Boden wurden bereits in verschiedenen Studien unter 
Anwendung der Isotopentechnik untersucht (Schneider \& Schmitz 1989, Simard et al. 1997, Fitter et al. 1998). Die Versorgung der Ektomykorrhiza mit Kohlenstoff aus dem Transport durch die Pflanze wurde an Hand radioaktiver Markierung bereits 1957 von Melin und Nilsson beobachtet. Sie zeigten, dass nach ${ }^{14} \mathrm{CO}_{2}$-Markierung von Kiefernblättern nach kurzer Zeit $6 \%$ des ${ }^{14} \mathrm{C}$ im Spross und $11-15 \%$ des ${ }^{14} \mathrm{C}$ in den Wurzelspitzen akkumuliert wurde. Bei einer einstündigen ${ }^{14} \mathrm{CO}_{2}$-Begasung von jungen Kiefern wurde beobachtet, dass nach 8 Stunden bereits $91-94 \%$ des ${ }^{14} \mathrm{CO}_{2}$ in Spross und Wurzeln vorgefunden wurden (Shiroya et al. 1962). In Bezug auf ${ }^{13} \mathrm{CO}_{2}$ konnte sogar bereits nach einer Stunde nach Begasung der Transport von $\mathrm{C}$ in die Wurzeln beobachtet werden (Ghirardo et al. 2011).

In dieser Arbeit wurde festgestellt, dass Buchen und Eschen im Freiland unterschiedliche Strategien bezüglich des Kohlenstofftransports und der Anreicherung aufwiesen (Kapitel 3). Beide Baumarten zeigten stärkere Anreicherung von Kohlenstoff in ihren Wurzelspitzen als in ihren Feinwurzeln, wobei der Transport in den Wurzelbereich in der ektomykorrhizerten Buche schneller erfolgte, die Anreicherung in der endomykorrhizierten Esche jedoch höher war. In der hier vorliegenden Untersuchung wurden weitere Laubbaumarten einbezogen, die mit Ektomykorrhizen und Endomykorrhizen vergesellschaftet sind. Neben der Buche und der Esche wurden endomykorrhizierter Bergahorn (Acer pseudo-platanus) und ektomykorrizhierte Linde (Tilia cordata) untersucht. In einem Gewächshausversuch wurden die Bedingungen in Bezug auf Lichtverhältnisse, Temperatur, Luftfeuchtigkeit und Wasser sowie die ${ }^{13} \mathrm{C}$-Begasung über einen relativ langen Markierungszeitraum von zwei Vegetationsperioden weitgehend konstant gehalten. Im Anschluss an eine ${ }^{13} \mathrm{C}$-Begasung wurde eine radioaktive Kohlenstoff-Pulsmarkierung durchgeführt. Mit dieser Versuchsanordnung sollte die Frage untersucht werden, ob die Mykorriza und Rhizosphäre durch länger- oder kurzfristige Kohlenstoffressourcen gespeist wird. 
Das ${ }^{13} \mathrm{CO}_{2}$-Begasungsexperiment wurde im Gewächshaus der Abteilung Forstbotanik und Baumphysiologie des Büsgen-Instituts der Universität Göttingen und die ${ }^{14} \mathrm{CO}_{2}$-Begasung im Labor für Radioisotope (LARI) durchgeführt.

\subsubsection{Vorbereitung}

Am 20.04. und 01.05.2011 wurden insgesamt rund 2000 Liter natürlicher Boden aus dem Nationalpark Hainich aus einer Waldfläche zwischen den Ortschaften Weberstedt und Craula (514'35" N 10³0'55" E) mit Spaten aus Bodentiefen bis 50 cm ausgehoben und nach Göttingen transportiert. Durch Sieben $(10 \mathrm{~mm}$ Maschenweite des Siebs) wurden größere Steine entfernt und der Boden durchmischt; ansonsten blieb der Boden naturbelassen. Für den Versuch wurden jeweils 150 noch nicht ausgetriebene einjährige Pflanzen mit Höhen von 30-50 cm der Baumarten Winterlinde (Tilia cordata), Rotbuche (Fagus sylvatica) und Bergahorn (Acer pseudoplatanus) verwendet. Die Bäume wurden Mitte April von einer Baumschule (Billenforst, Bösinghausen, Deutschland) geliefert und bis zum Versuchsbeginn in einem Kühlraum bei $4^{\circ} \mathrm{C}$ unter Ausschluss von Licht gelagert.

Am 02.05. wurden die Pflanzen teils einzeln in Töpfe oder im Verbund von jeweils 18 Pflanzen pro Baumart in Wannen gepflanzt. Die insgesamt 287 Einzeltöpfe (Meyer, Rellingen, Deutschland) hatten ein Volumen von jeweils 5 Litern $(17 \times 17 \mathrm{~cm}$ Kantenlänge oben, $15 \times 15 \mathrm{~cm}$ Kantenlänge unten, $18 \mathrm{~cm}$ hoch). In die Wannen (Meyer, Rellingen, Deutschland) wurden jeweils 18 Pflanzen einer Baumart gepflanzt mit jeweils 3 Wannen pro Baumart. Jede Wanne hatte ein Volumen von 92,5 Litern (42 cm Breite, $71 \mathrm{~cm}$ Länge, $31 \mathrm{~cm}$ Höhe) und die Pflanzen wurden nach folgendem Schema eingepflanzt (Abbildung 5.2.1): 


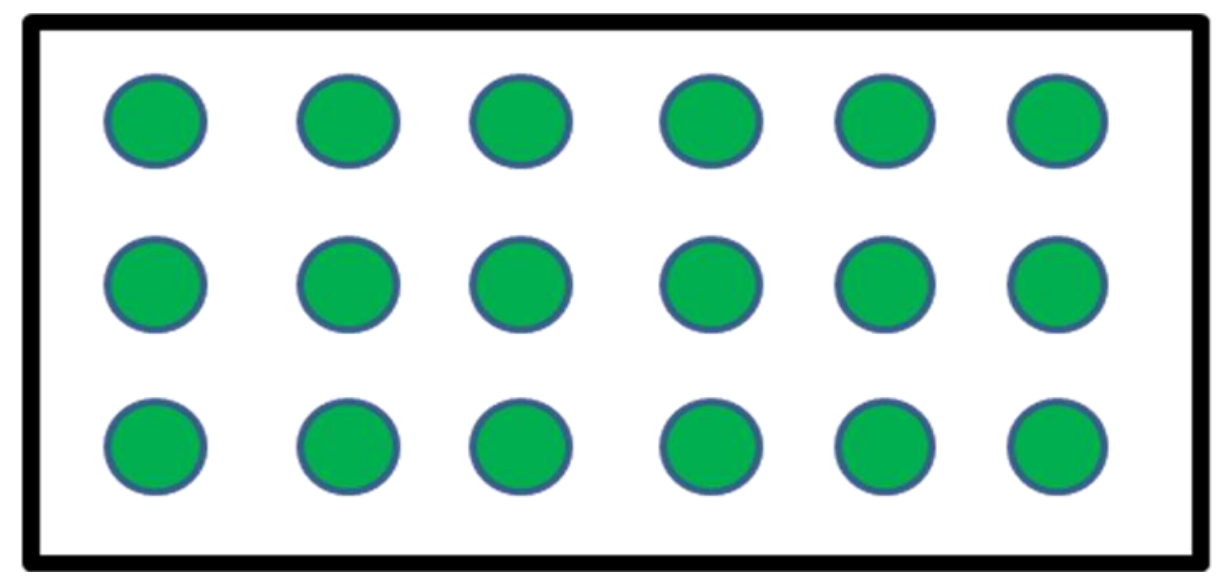

Abbildung 5.2.1: Pflanzenanordnung in den Wannen

Alle Pflanzen wurden mit kleinen Kunststoffschildern versehen und durchnummeriert. Die Einzeltöpfe wurden in Kontrollen und markierte Pflanzen aufgeteilt und in zwei getrennten Gewächshauskabinen untergebracht. Die Pflanzen in den insgesamt 9 Wannen wurden in die Kabine der zu begasenden Pflanzen gestellt.

Für den folgenden Versuch wurden die in Wannen gepflanzten Bäume nicht verwendet. Das Experiment mit Kombination von ${ }^{13} \mathrm{CO}_{2}-{ }^{14} \mathrm{CO}_{2}$-Markierung wurde an den Pflanzen in Einzeltöpfen durchgeführt.

Der Versuch erfolgte über einen Versuchszeitraum von zwei Vegetationsperioden mit einer langfristigen ${ }^{13} \mathrm{CO}_{2}$-Begasung in der Aufwuchsphase, die durch eine Winterruhe unterbrochen wurde und einer kurzfristigen ${ }^{14} \mathrm{CO}_{2}$-Begasung. Im Anschluss erfolgte die Ernte einen Tag, eine Woche sowie ein Jahr nach der ${ }^{14} \mathrm{CO}_{2}$-Begasung (Abbildung 5.2.2). 


\begin{tabular}{|c|c|c|c|}
\hline $\begin{array}{l}\text { Boden aus } \\
\text { Hainich } \\
\text { entnommen }\end{array}$ & $\begin{array}{l}\text { Ahorn, Buche } \\
\text { und Linde } \\
\text { in Boden gepflanzt }\end{array}$ & $\begin{array}{l}\text { Aufwuchsphase } \\
\text { ohne } \\
\text { Behandlung }\end{array}$ & $\begin{array}{l}\text { Beginn der }{ }^{19} \mathrm{CO}_{2} \text {-Begasung } \\
\text { in Gewächshauskammern }\end{array}$ \\
\hline 01.05 .2011 & 02.05 .2011 & $03.05 .-23.06 .2011$ & 06.07 .2011 \\
\hline $\begin{array}{l}\text { Ende der ersten } \\
{ }^{13} \mathrm{CO}_{2}= \\
\text { Begasungs- } \\
\text { phase }\end{array}$ & $\begin{array}{l}\text { Umstellung auf } \\
\text { Kurztagbedingungen }\end{array}$ & $\begin{array}{l}\text { Licht- und Temperatur- } \\
\text { regelung } \\
\text { vollständig eingestellt }\end{array}$ & $\begin{array}{l}\text { Pflanzen zum Uberwintern } \\
\text { in Kaltegewachshaus } \\
\text { Verbracht }\end{array}$ \\
\hline 26.11 .2011 & 26.11 .2011 & 12.12 .2011 & 20.12 .2011 \\
\hline $\begin{array}{l}\text { Beginn der } \\
\text { zweiten }{ }^{13} \mathrm{CO}_{2}^{-} \\
\text {Begasung in } \\
\text { Gewãchshaus- } \\
\text { kammern }\end{array}$ & $\begin{array}{l}\text { Ende der zweiten } \\
{ }^{13 \mathrm{CO}_{2}-} \\
\text { Begasungsphase }\end{array}$ & $\begin{array}{l}{ }^{14} \mathrm{CO}_{2} \text {-Pulsmarkierung } \\
\text { über } 16 \text { Stunden }\end{array}$ & Ernte ,1 Tag ${ }^{\circ}$ \\
\hline 25.04 .2012 & 05.07 .2012 & $\begin{array}{l}24.07 .2012 \text { 1. Kohorte } \\
25.07 .2012 \text { 2. Kohorte } \\
26.07 .2012 \text { 3. Kohorte }\end{array}$ & $\begin{array}{l}25,07.2012 \\
\text { 1. Kohorte }\end{array}$ \\
\hline Ernte .1 Woche & Ernte „1 Jahr & & \\
\hline $\begin{array}{l}02.08 .2012 \\
\text { 2. Kohorte }\end{array}$ & $\begin{array}{l}\text { 30.07.2013 } \\
\text { 3. Kohorte }\end{array}$ & & \\
\hline
\end{tabular}

Abbildung 5.2.2 Zeitschema des Versuchsablaufs

\subsection{2 ${ }^{13} \mathrm{CO}_{2}$ - und ${ }^{14} \mathrm{CO}_{2}$-Begasung}

Die Pflanzen wurden vom 24.06.2011 bis zum 05.07.2011 mit natürlichem $\mathrm{CO}_{2}$-Gas (Reinheit 99,2 \%) (AirLiquide, Düsseldorf, Deutschland) begast (1100 ppm) und nach Bedarf mit Leitungswasser versorgt. Die Begasung der Pflanzen mit ${ }^{12} \mathrm{CO}_{2}$-Gas diente der Kontrolle der Funktionsfähigkeit der Begasungsanlage und der Kabine. Bei der Begasungsanlage handelte es sich um einen Eigenbau der Abteilung Forstbotanik (Büsgen-Institut, Universität Göttingen). Die Kontrollpflanzen blieben unbehandelt.

Nachdem alle drei Baumarten vollständig ausgetrieben waren, wurde die Begasung mit 1,5\%-13 $\mathrm{CO}_{2}$-Gas (Euriso-Top, Saarbrücken, Deutschland) am 06.07.2011 begonnen, damit die Pflanzen unter der ${ }^{13} \mathrm{CO}_{2}$-Atmosphäre aufwachsen konnten. Während der Begasung über die Vegetationsperiode wurden die Pflanzen nach Bedarf mit Leitungswasser versorgt. Zu Beginn des Experimentes betrug die Anzahl an Pflanzen in Einzeltöpfen bei Ahorn 15 für Kontrolle und 30 für Markierung, bei Buche 15 für Kontrolle und 30 für Markierung sowie bei Linde 15 für Kontrolle und 30 für Markierung. Bei allen drei Baumarten war sichtbar, dass einige Pflanzen zu diesem Zeitpunkt keinerlei Austrieb zeigten und wurden aus dem Experiment 
entfernt. An den verbliebenen Einzelpflanzen wurde das Experiment in folgender Verteilung durchgeführt: 13 Ahorne als Kontrollen sowie 24 Ahorne für die Markierung, 8 Buchen als Kontrollen und 16 Buchen für die Markierung; 15 Linden als Kontrollen sowie 24 Linden.

In beiden Gewächshauskabinen RM 1 (Kontrolle) und RM 2 (Begasung) wurden kontrollierte Bedingungen von $65 \%$ relativer Luftfeuchte und $20-22^{\circ} \mathrm{Celsius}$ unter Langtagbedingungen (16 Stunden Licht durch künstliche Bestrahlung) gehalten, wobei die Temperatur an sehr warmen Tagen mit starker Sonnenstrahlung bis $26^{\circ} \mathrm{C}$ anstieg. Die Begasung erfolgte mit $1100 \pm 100$ ppm (parts per million) $\mathrm{CO}_{2}$. Am 8.8.2011 wurde eine Buchenpflanze geerntet, um die ${ }^{13} \mathrm{C}$-Signatur in den Feinwurzeln zu überprüfen. Mit dem Laubfall wurde die Begasung in der Vegetationsperiode 2011 am 26.11.2011 unterbrochen und die Pflanzen von Langtagbedingungen auf Kurztagbedingungen (8 Stunden Licht und Senkung der Temperatur von $22^{\circ}$ auf $17^{\circ}$ Celsius) auf die Winterperiode vorbereitet. Abgefallenes Laub wurde je nach Anfallen nach Baumarten getrennt eingesammelt, gegebenenfalls getrocknet, gewogen und eingelagert. Nach der ersten Versuchsperiode wurden die Pflanzen am 01.12.2011 nach Wurzelhalsdurchmesser und Höhe bonitiert. Am 12.12.2011 wurden Licht- und Temperaturregelungen in den Gewächshauskabinen vollständig eingestellt und die Pflanzen am 20.12.2011 zur Überwinterung in ein Kältegewächshaus $\left(6-8{ }^{\circ} \mathrm{C}\right)$ gebracht. Die schrittweise Anpassung an winterliche Bedingungen sollte Stressreaktionen bei den Pflanzen verhindern. Da die jungen Pflanzen an kontrollierte Bedingungen gewöhnt waren, wurde aus diesem Grund auf eine Überwinterung im Freien verzichtet. Während des Winters erhielten die Pflanzen, je nach Bedarf, $1 \mathrm{x}$ bis $2 \mathrm{x}$ mal wöchentlich Leitungswasser.

Nachdem die Pflanzen in der darauffolgenden Vegetationsperiode 2012 im Überwinterungsgewächshaus wieder ausgetrieben waren und die Pflanzen zurück in die Gewächshauskabinen verbracht wurden, wurde die Begasung unter gleichen Bedingungen wie im Vorjahr ab dem 25.04.2012 fortgesetzt. Dabei betrug die relative Luftfeuchte wieder $65 \%$ und die Temperatur $20-22^{\circ} \mathrm{C}$ in den Gewächshauskabinen. Am 29.05.2012 wurden drei Buchen geerntet, um die Einlagerung des zugeführten ${ }^{13} \mathrm{C}$ in den Pflanzenkompartimenten Blätter, Holz, Rinde, Grob- sowie Feinwurzeln zu überprüfen. Die Begasung mit ${ }^{13} \mathrm{C}$ endete am 05.07.2012. 
Im Anschluss an die ${ }^{13} \mathrm{CO}$-Begasung der Pflanzen wurde eine ${ }^{14} \mathrm{CO}_{2}$-Begasung durchgeführt. Auf Grund der Kammerkapazität wurden die insgesamt 45 markierten Einzelpflanzen in drei Durchgängen an drei aufeinanderfolgenden Tagen einem ${ }^{14} \mathrm{CO}_{2}$-Begasungspuls über eine Lichtphase von 16 Stunden ausgesetzt. Dazu wurden am 23.07.2012 alle Pflanzen in den Vorraum der Kammer gebracht, um die Pflanzen an die dort herrschenden Bedingungen zu akklimatisieren. Am 24.07.2012 wurden 15 Pflanzen (je fünf jeder Art) in den Töpfen in die Kammer gestellt und für eine Lichtphase von 16 Stunden begast. Das verwendete Gas wurde durch das Zusammenfügen von ${ }^{14} \mathrm{C}$-Natriumcarbonat und Milchsäure hergestellt. Bei der chemischen Reaktion von ${ }^{14} \mathrm{C}$-markiertem Natriumcarbonat (Moravek Biochemicals, Brea, USA) mit 90\%-iger Milchsäure (AppliChem, GmbH, Darmstadt, Deutschland) entsteht ${ }^{14} \mathrm{CO}_{2}$-markiertes Kohlenstoffgas. Radioaktive Markierungsexperimente müssen innerhalb einer sogenannten Freigrenze der radioaktiven Konzentration bleiben. Hier wurde die höchste zugelassene Konzentration von $185 \mathrm{MBq}{ }^{14} \mathrm{CO}_{2}$ (bezogen auf $5 \mathrm{ml} 2 \mathrm{~N} \mathrm{NaOH}$, in dem das Bariumcarbonat (ARC0109A, American Radiolabeled chemicals, Saint Louis, USA) gelöst war, verwendet; dies entsprach $22200000 \mathrm{dpm} / \mu \mathrm{l} \mathrm{NaOH}$. Für den ersten Durchgang wurden $0,25 \mathrm{ml}$ der radioaktiven Substanz und für die beiden folgenden Durchgänge jeweils $0,15 \mathrm{ml}$ radioaktiver Substanz verwendet. Zur Bildung des ${ }^{14} \mathrm{CO}_{2}$ wurden jeweils 1 Liter 0,5 M Milchsäure benötigt. Daraus ergab sich ein freigesetztes Volumen von ${ }^{14} \mathrm{CO}_{2}$ von 11,2 Liter pro Durchgang.

Das ${ }^{14} \mathrm{CO}_{2}$-markierte Gas entwickelte sich aus der Zusammenführung von Natriumcarbonat mit der Milchsäure. Beide Substanzen befanden sich jeweils außerhalb der Kammer in 1 L-Messkolben, die über einen Schlauch mit einer Dosierpumpe verbunden waren. Eine weiterführende Schlauchverbindung verbrachte die Substanzen zum Vermischen in den Dosierkolben. (Eine detaillierte Beschreibung der Versuchskammer und ihrer Funktion ist der Masterarbeit „Allokation von Kohlenstoff und Stickstoff in den Wurzelraum junger Buchen (Fagus sylvatica L.) und Eschen (Fraxinus excelsior L.) von Silke Ammerschubert, im selben Institut im Kontext des Graduiertenkollegs 1086 angefertigt wurde, zu entnehmen.) Aus diesem gelangte das entstandene Gas aus der Reaktion

$$
\mathrm{Na}_{2}{ }^{14} \mathrm{CO}_{3}+2 \mathrm{C}_{3} \mathrm{H}_{6} \mathrm{O}_{3}->2 \mathrm{NaC}_{3} \mathrm{H}_{5} \mathrm{O}_{3}+{ }^{14} \mathrm{CO}_{2}+\mathrm{H}_{2} \mathrm{O}
$$


in die Kammer. Die Konzentration betrug dabei $360 \mathrm{ppm}$ des freigesetzten $\mathrm{CO}_{2}$ bezogen auf das Kammervolumen. Der ${ }^{14} \mathrm{CO}_{2}$-Puls wurde pro Kohorte einmalig gegeben und die Pflanzen über 16 Stunden in der ${ }^{14} \mathrm{CO}_{2}$-Atmosphäre inkubiert. Die erste Kohorte wurde am Ende der Begasung am 25.07. aus der Kammer genommen. Die markierten Pflanzen wurden zurück in die Gewächshauskabine der Forstbotanik gebracht und konnten dort verbleiben, da ihre Markierung unterhalb der Freigrenze lag. In gleicher Art und Weise wurden zwei weitere Kohorten inkubiert.

Die Pflanzen der ersten Kohorte am 24.07.2012 wurden am 25.07.2012 nach 1 Tag, die Pflanzen der zweiten Kohorte am 02.08.2012 nach 1 Woche und die der dritten Kohorte am 30.07.2013 nach $1 \mathrm{Jahr}$ wie folgt geerntet. Die unbehandelten Kontrollen wurden am 01.08.2012 geerntet.

\subsubsection{Ernte}

Nach folgendem Ablauf wurde jede Pflanze geerntet: Alle Blätter der Pflanzen wurden gezählt und zur Bestimmung der spezifischen Blattfläche wurden drei Blätter (jeweils eines oben, mittig und unten) entnommen, gewogen, fotokopiert und getrocknet. Aus der Summe der drei Blätter ergab sich das Trockengewicht der SLAProbe. Die restlichen Blätter wurden abgesammelt, gewogen und getrocknet, danach erneut gewogen. Daraus wurde die Gesamtblattfläche pro Pflanze wie folgend ermittelt:

Gesamtblattfläche

$=\frac{\text { Fläche der SLA-Probe }\left(\mathrm{cm}^{2}\right)}{\text { * }}$ Gesamtgewicht der Blätter $(\mathrm{g})$ Gewicht der SLA-Probe (g)

Der Spross wurde bis auf den untersten $1 \mathrm{~cm}$, in kleinere Stücke geteilt, gewogen und getrocknet sowie danach erneut gewogen. Die untersten $1 \mathrm{~cm}$ des Sprosses wurden nach Holz und Rinde getrennt und für weitere Analysen bei minus $20^{\circ} \mathrm{Celsius}$ eingefroren. Die verbleibende Wurzel wurde aus dem Topf entnommen, locker anhaftender Boden vorsichtig abgeschüttelt und direkt an Wurzeln anhaftender Rhizosphärenboden mit einem Zahnstocher vorsichtig entnommen, gewogen und getrocknet; danach erneut gewogen. Der restliche Boden wurde vermischt, als Ganzes gewogen, eine Probe entnommen, diese gewogen, getrocknet und danach erneut gewogen. Die Wurzel wurde behutsam mit Wasser gewaschen und das Frischgewicht bestimmt. Danach wurden Grob- und Feinwurzeln voneinander 
getrennt und jeweils gewogen. Von den Grobwurzeln wurde je ein Aliquot zur Trockengewichtsbestimmung getrocknet und eines für weitere Analysen bei minus $20^{\circ}$ Celsius eingefroren. Die Feinwurzel wurde zerschnitten, homogen vermischt und in zwei Aliquots (eines für Mykorrhizaanalyse und eines für weitere Analysen) eingefroren.

\subsubsection{Mykorrhiza}

\subsubsection{Probennahme von Wurzelspitzen}

Zur Bestimmung von ${ }^{13} \mathrm{C}$ und ${ }^{14} \mathrm{C}$ in den Mykorrhizen von Buche und Linde bzw. in den Wurzelspitzen des Ahorns wurden Wurzelspitzen gesammelt. Für die Analyse der Ektomykorrhizaarten an Buche und Winterlinde wurde jeweils ein Aliquot der Feinwurzelproben für die Probennahme aufgetaut, die Probe in eine Glasschale gegeben und mit feuchtem Zelltuch abgedeckt, um ein Austrocknen der Feinwurzeln zu vermeiden. Um eine mögliche Auswaschung des markierten Kohlenstoffes in den entsprechen Proben zu verhindern, wurde den Feinwurzeln kein Wasser zugegeben, sondern das feuchte Zelltuch gegebenenfalls ausgetauscht. Die Kontrollproben wurden in derselben Art und Weise behandelt, obwohl bei diesen keine mögliche Auswaschung von ${ }^{13} \mathrm{C}$ bzw. ${ }^{14} \mathrm{C}$ zu befürchten war. Aus der aufgetauten Probe wurde eine kleine Menge herausgenommen und unter dem Stereomikroskop (M 205 FA, Leica, Wetzlar, Deutschland) betrachtet. Die ektomykorrhizierten Wurzelspitzen wurden mit Hilfe einer Pinzette von der Wurzel abgenommen, in EppendorfReaktionsgefäßen gesammelt und für die anschließende Isotopenbestimmung in einem Trockenschrank bei $40^{\circ}$ Celsius für eine Woche (Memmert, Schwabach, Deutschland) getrocknet. Beim Ahorn wurden die Wurzelspitzen ebenfalls mit Hilfe einer Pinzette von der Wurzel abgenommen, in Eppendorf-Reaktionsgefäßen gesammelt und getrocknet. Abbildung 5.2.3 zeigt die jeweiligen Bereiche der Wurzeln an ekto- und endomykorrhizierten Wurzeln. 

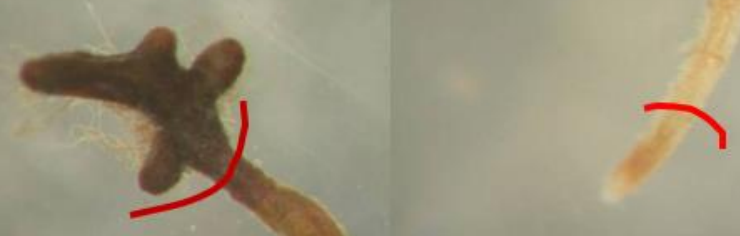

Abbildung 5.2.3: Entnommene Wurzelspitzen an ektomykorrhizierten (a) und endomykorrhizierten Wurzeln (B); rote Striche bezeichnen den Bereich, an dem die Wurzelspitze abgetrennt wurde

Die Bestimmung der Isotopen erfolgte danach als Mischung über die jeweilige Baumart aus verschiedenen Morphotypen pro Baum sowie beim Ahorn als Pool aus Wurzelspitzen pro Baum.

Auf Grundlage der gezählten Wurzelspitzen wurden die Mykorrhizierungsrate der Buchenwurzeln (a) sowie die Vitalitätsrate der gezählten Wurzelspitzen (b) bestimmt.

a) Mykorrhizierungsrate (\%)

$=\frac{\text { Anzahl vitaler mykorrhizierter WS }}{\text { Anzahl vitaler nicht-mykorrhizierter WS + vitaler mykorrhizierter WS }}{ }^{*}$

b) Vitalitätsrate (\%)

$=\quad \underline{\text { Anzahl vitaler nicht-mykorrhizierter WS }+ \text { vitaler mykorrhizierter WS }}{ }^{*} 100$ Anzahl aller gezählten WS

Die optische Ansprache der vitalen Wurzeln von Buche, Linde und Ahorn erfolgte an Hand der Farbe und Oberflächenbeschaffenheit. Vitale Wurzeln zeigen eine glatte Oberflächenstruktur. Nicht-vitale Wurzeln sehen durch den Wasserverlust an ihrer Oberfläche „verschrumpelt“ aus.

Um die pilzlichen Strukturen in den Ahornfeinwurzeln erkenntlich zu machen, wurden sie mit Lactophenol (Merck, Darmstadt, Deutschland) eingefärbt. Mit der Methode nach Schmitz et al. (1991) färben sich die pilzlichen Strukturen innerhalb der Feinwurzel blau. Dazu wurden die Ahornwurzeln mit einer 10\%igen Kalilauge $(\mathrm{KOH})$ für 50 Minuten bei $90^{\circ} \mathrm{C}$ in einem Thermoblock entfärbt. Nach Waschen mit destilliertem Wasser wurden die Feinwurzeln für 15 Minuten in 3,7\%iger Salzsäure bei Raumtemperatur angesäuert. Nach weiterem Waschen mit destilliertem Wasser 
wurden die Feinwurzeln bis zu 2 Minuten in einer Lactophenol-Lösung (1 $\mathrm{g} / \mathrm{l}$ destilliertes Wasser) eingefärbt. Überschüssiger Farbstoff wurde entfernt, indem die Feinwurzeln danach 60 Minuten in einer sauren Glycerinlösung (50 ml Glycerin, 45 $\mathrm{ml}$ destilliertes Wasser, $5 \mathrm{ml}$ 1\%ige Salzsäure) bei Raumtemperatur inkubiert wurden.

Die Erfassung der arbuskulären Mykorrhiza am Bergahorn erfolgte nach der "magnified intersection method" nach McGonigle et al. (1990), bei der die Mykorrhizierung der Wurzel an Hand ihrer Anzahl an arbuskulär-spezifischen Hyphen, Vesikel und Arbuskeln charakterisiert wird. Pro Pflanze wurden 3 Feinwurzeln, ausgehend von der Wurzelspitze in Richtung der Grobwurzel am Mikroskop (Axioplan inklusive Digitalkamera AxioCam und Software Axiovision, Zeiss, Oberkochen, Deutschland) ausgezählt. Bei der angewandten Methode wird über das Okular ein quadratisches Raster mit 100 Einheiten über die angefärbten Wurzeln, die nach der Anfärbung gequetscht wurden, gelegt. In den Einheiten (hier als Abschnitte bezeichnet) wurde das Auftreten von Hyphen, Arbuskeln, Vesikeln bzw. das Auftreten nicht indentifizierbarer Wurzelmasse und leere Abschnitte dokumentiert. Die Kolonisierung mit Hyphen, Vesikeln und Arbuskeln wurde an Hand folgender Formeln berechnet.

a) Kolonisierungsrate Hyphen (\%)

$=\frac{\text { Summe an Abschntten mit Hyphe }}{\text { Summe an Abschnitten mit Wurzel }}{ }^{*} 100$

b) Kolonisierungsrate Arbuskel (\%)

$=\frac{\text { Summe an Arbuskel mit Hyphe }}{\text { Summe an Abschnitten mit Wurzel }}{ }^{*} 100$

c) Kolonisierungsrate Vesikel (\%)

$=\frac{\text { Summe an Arbuskel mit Vesikel }}{\text { Summe an Abschnitten mit Wurzel }}{ }^{* 100}$ 
Die Gesamtmykorrhizierung ergab sich aus der Summe aus den Abschnitten mit Kolonisierungen mit Hyphen, Arbuskeln und Vesikel im Verhältnis zur gezählten Anzahl von Abschnitten.

Gesamtmykorrhizierung

$=\underline{\text { Kolonisierung Hyphe }+ \text { Kolonisierung Arbuskel }+ \text { Kolonisierung Vesikel }}{ }^{*}$ 100 Summe aller gezählten Abschnitte

\subsubsection{Isotopenanalyse}

\subsubsection{1 ${ }^{13} \mathrm{C}$ und ${ }^{14} \mathrm{C}$ in verschiedenen Kompartimenten}

Die Bestimmung der Isotopensignaturen erfolgte getrennt nach ${ }^{13} \mathrm{C}$ und ${ }^{14} \mathrm{C}$, wobei die Messungen im Kompetenzzentrum für Stabile Isotope (KOSI) der Universität Göttingen für das stabile Isotop ${ }^{13} \mathrm{C}$ und im Labor für Radioaktive Isotope (LARI) für das radioaktive Isotop ${ }^{14} \mathrm{C}$ durchgeführt wurden. Zur Vorbereitung der Messungen wurden die getrockneten Proben von Blättern, Spross, Grob- und Feinwurzeln sowie der Rhizosphären- und des Mischbodens gemahlen (Retsch, Haan, Deutschland) und daraus Aliquots entnommen. Die Wurzelspitzen wurden auf Grund ihrer geringen Mengen als Mykorriza (bei Buche und Linde) beziehungsweise als Wurzelspitzen (bei Ahorn) pro Baum als Einzelprobe zusammengefasst und ohne vorheriges Mahlen eingewogen. Für die Bestimmung der ${ }^{13} \mathrm{C}$-Signatur wurde jeweils $1 \mathrm{mg}$ des Kompartimentes in Zinnkartuschen (4x6 mm, Hekatech, Wegberg, Deutschland) eingewogen; bei der Mykorrhiza und den Wurzelspitzen auf Grund der geringen Menge weniger. Die Menge betrug dabei zum Teil nur $200 \mu \mathrm{g}$. Die ${ }^{13} \mathrm{C}-$ Konzentration wurde mit einem Isotopenmassenspektrometer Delta plus (Finnigan MAT, Bremen, Deutschland) und einem Elementaranalysator NA 1500 (Fisons-Instruments, Rodano, Milano, Italien) bestimmt und um die ${ }^{13} \mathrm{C}$-Signatur zu bestimmen, wurde das Verhältnis zwischen ${ }^{13} \mathrm{C}$ und ${ }^{12} \mathrm{C}$ wie folgend ermittelt:

Isotopensignatur Kohlenstoff $(\partial \% 0)$ )

$\partial{ }^{13} \mathrm{C}(\%)=\frac{R \text { Probe }- \text { R Standard }}{R \text { Standard }} * 1000$ 
mit $R=$ Verhältnis des schweren Isotops $\left({ }^{14} \mathrm{C}\right)$ zu leichtem Isotop $\left({ }^{13} \mathrm{C}\right)$

und Carbonat (Belemnite) aus der PeeDee-Formation (V-PDB) als Standard

(Kendall \& Caldwell,1998)

Die Signatur von ${ }^{14} \mathrm{C}$ in den Proben wurde an Hand der Flüssigszintillationsmessung bestimmt. Dazu wurden jeweils 0,1 - 0,3 g Blattmaterial, 0,1 - 0,2 g Sprossmaterial, 0,1 - 0,2 g Fein- bzw. Grobwurzelmaterial, ca. 0,01 g Mykorrhiza bzw. $1 \mathrm{~g}$ Bodenmaterial verwendet. Zur Bestimmung wurden die trockenen und gemahlenen Proben unter Zugabe von Sauerstoff in einem Verbrennungsofen Ox500 (Zinsser Analytic, Frankfurt, Deutschland) zu Wasser und Kohlendioxid verbrannt. Das aufgefangene Kohlendioxid wurde in einem Szinzillationsliquid Oxysolve C 400 (Zinsser, Frankfurt, Deutschland; $1,8 \mathrm{CO}_{2}$ auf $16 \mathrm{ml}$ Szintillationsliquid (40-60\% 3Methoxypropylamin, 40-60\% 1,2,4-Trimethylbenzen (Sigma-Aldrich, Taufkirchen, Deutschland))) in einem Flüssigszintillationszähler Packard Tricarb 2800 (Perkin Elmer, Rodgau, Deutschland) gemessen. Die Signatur des radioaktiven Isotopes wird an Hand der Zerfälle des Isotopes pro Minute in der entsprechenden Materie festgestellt.

\subsubsection{Statistik}

Die erhobenen Daten der biometrischen Parameter und die Messdaten der ${ }^{13} \mathrm{C}$ - und ${ }^{14} \mathrm{C}$-Markierung wurden mit Hilfe von Microsoft Excel (Windows, Redmond, USA) und OriginPro 8.5 (OriginLab Corporation, Northampton, USA) statistisch analysiert. Die Daten wurden an Hand von Shapiro-Wilk- bzw. Kolmogorv-Smirnov-Test auf Normalverteilung getestet. An Hand der Daten wurden für die biometrischen Parameter und Isotopenuntersuchungen auf Grundlage von Two-Way-ANOVA mit den Faktoren Baumart und Erntetag p-Werte berechnet. 


\subsection{Ergebnisse}

\subsubsection{Biometrische Analysen}

Die Versuchspflanzen der drei Baumarten wurden an Hand ihrer biometrischen Parameter analysiert. Hierbei wurden die Parameter Pflanzenhöhe, Wurzelhalsdurchmesser (WHD) und Blattanzahl sowie die jeweiligen Trockengewichte der einzelnen Kompartimente Blätter, Spross und Wurzeln hinzugezogen. In Bezug auf Buche und Linde wurde die Mykorrhizierungs- und Vitalitätsrate der Wurzeln und beim Ahorn die Kolonisierung der Wurzeln mit Arbuskeln, Vesikeln und Hyphen bestimmt.

In Bezug auf die biometrischen Daten wurden die Kontrollen als Zeitpunkt „0“ bezeichnet und am 30.07.2012 geerntet. Die Daten des Erntezeitpunktes nach einem Tag und nach einer Woche wurden zu „2012“ zusammengefasst und die Daten des Erntezeitpunktes nach einem Jahr als Zeitpunkt „2013“ bezeichnet.

Im Folgenden werden die Gesamttrockenmasse sowie Gesamtblattfläche sowie die Mykorrhizierung der Pflanzen gezeigt; die weiteren erhobenen Parameter Höhe, Wurzelhalsdurchmesser, Blattanzahl und die Trockengewichte von Blätter, Spross, Grob- und Feinwurzeln sind dem Anhang zu entnehmen.

Es zeigte sich, dass sich die untersuchten Baumarten in Bezug auf die Gesamttrockenmasse signifikant unterschieden (Abbildung 5.3.1). Die Gesamttrockenmasse des Ahorns betrug in den Kontrollen $46 \pm 2,2 \mathrm{~g}$ und in den markierten Pflanzen 89,5 \pm 9,7 g in 2012 und 103,4 \pm 10,1 g in 2013. Bei der Buche betrug das Gesamttrockengewicht $47 \pm 11,2 \mathrm{~g}$ in den Kontrollen und in den markierten Pflanzen 34,7 $\pm 2,3$ in 2012 und 86,8 \pm 14,4 g in 2013. Das Gesamttrockengewicht der Linde betrug in den Kontrollen 35,6 $\pm 2,8 \mathrm{~g}$ und in den markierten Pflanzen 42,7 $\pm 3,4 \mathrm{~g}$ in 2012 und 67,9 \pm 7,5 g in 2013. In den markierten Pflanzen zeigte der Ahorn die größte Gesamttrockenmasse (Abbildung 5.3.1). 


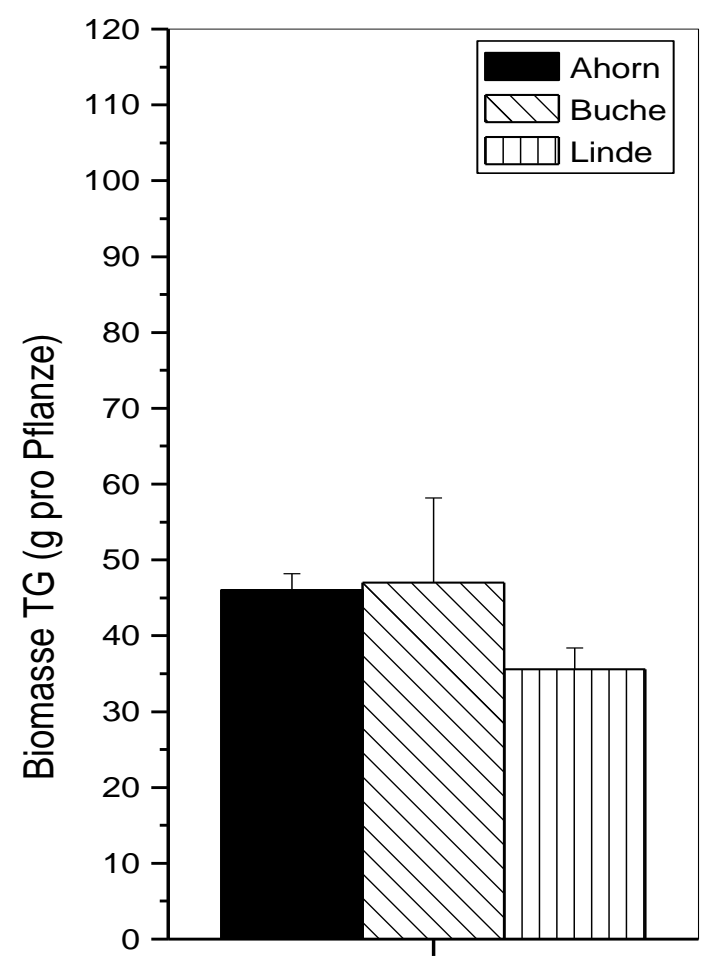

Erntezeitpunkt (Kontrollen)

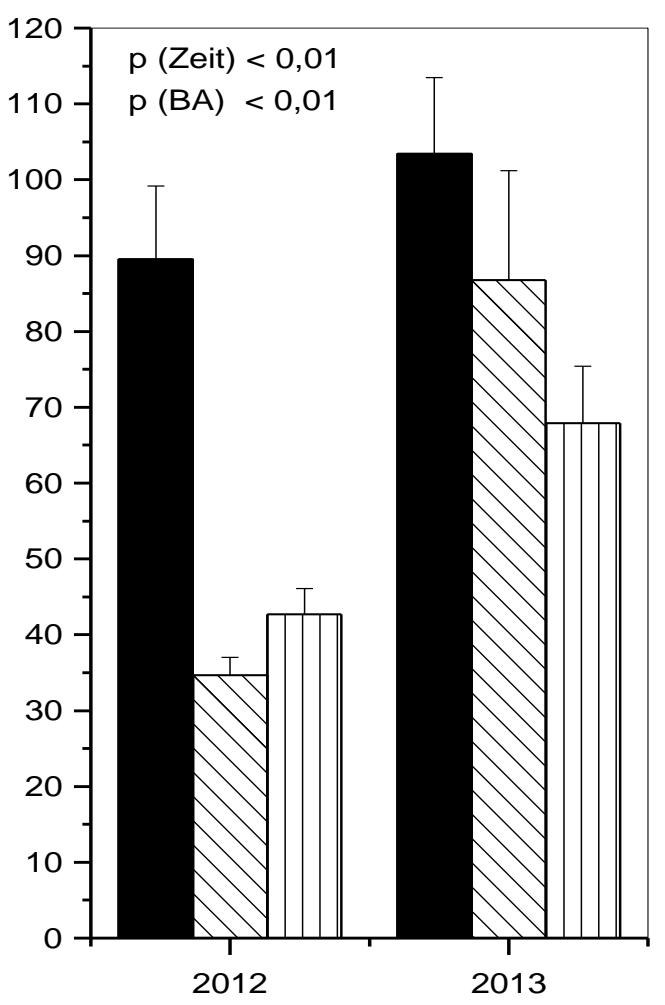

Erntezeitpunkt (Markierte Pflanzen)

Abbildung 5.3.1: Trockenmasse (TG) der gesamten Pflanze in den Kontrollen und beiden ${ }^{13} \mathrm{C}$ begasten Vegetationsperioden 2012 und 2013, entsprechend den Erntezeitpunkt von 1 Tag und 1 Woche (2012) und 1 Jahr nach der ${ }^{14} \mathrm{C}-$ Markierung (2013); Daten zeigen MW $\pm \mathrm{SE} ; \mathrm{n}=3-10$, $\mathrm{BA}=$ Baumart; Two- Way-ANOVA mit den Faktoren Zeit $(0,2012,2013)$ und Baumart (Ahorn, Buche, Linde)

Die Gesamtblattfläche pro Pflanze betrug durchschnittlich beim Ahorn zwischen 1462 $\pm 159 \mathrm{~cm}^{2}$ bei den Kontrollen und $3602 \pm 269 \mathrm{~cm}^{2}$ bei den markierten Pflanzen am letzten Erntezeitpunkt. Bei der Buche betrug die Blattfläche in den Kontrollen $2207 \pm$ $614 \mathrm{~cm}^{2}$, in $20121562 \pm 101 \mathrm{~cm}^{2}$ und am letzten Erntezeitpunkt $2658 \pm 622 \mathrm{~cm}^{2}$. Bei der Linde zwischen wurde eine Blattfläche zwischen $1705 \pm 190 \mathrm{~cm}^{2}$ in den Kontrollen und $3636 \pm 185 \mathrm{~cm}^{2}$ am letzten Erntezeitpunkt gemessen (Abbildung 5.3.2). Bei allen Baumarten war die gesamte Blattfläche pro Pflanze am Versuchsende am höchsten. Ein signifikanter Unterschied in der Blattfläche pro Pflanze wurde zwischen den Baumarten jedoch nicht beobachtet $(p=0,16)$. 


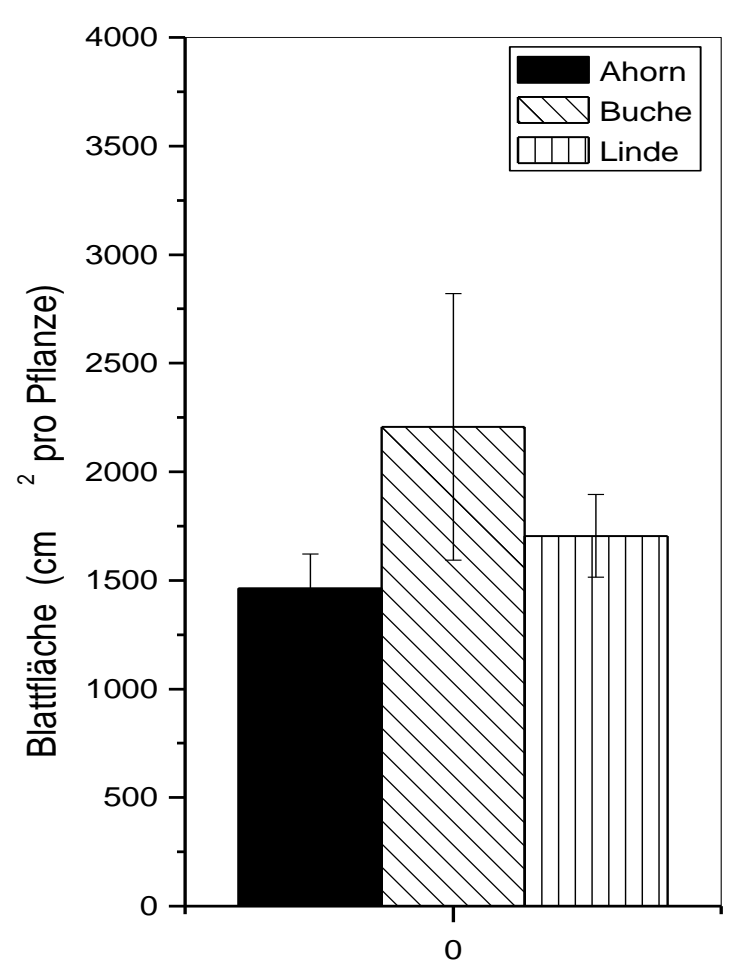

Erntezeitpunkt (Kontrollen)

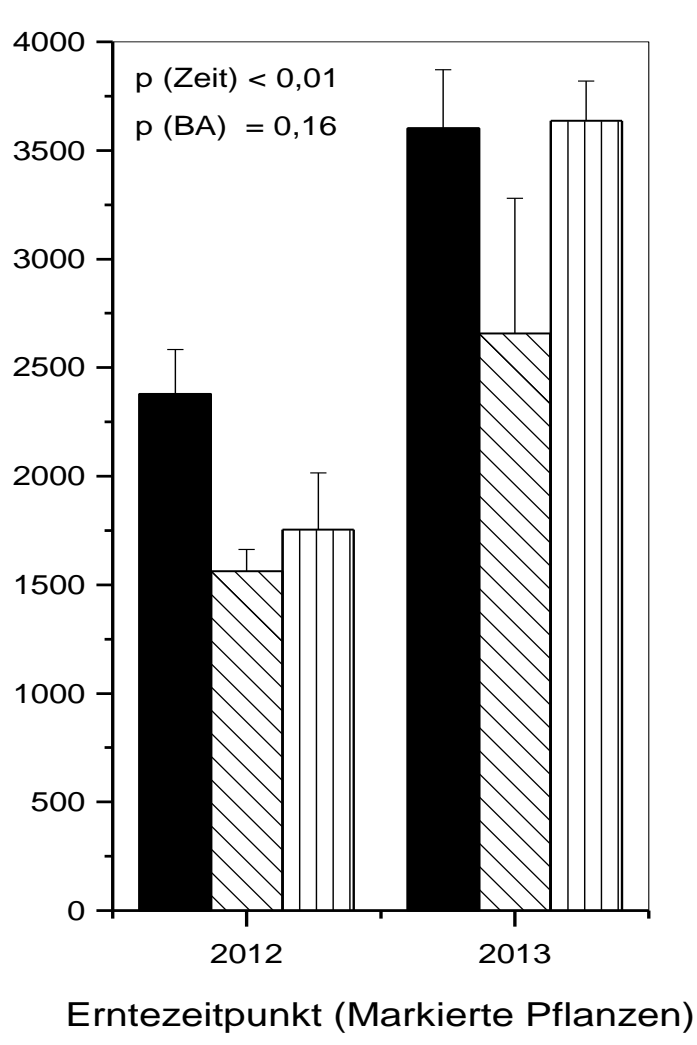

Erntezeitpunkt (Markierte Pflanzen)

Abbildung 5.3.2: Blattfläche pro Pflanze der Baumarten in den Kontrollen (0) und beiden ${ }^{13} \mathrm{C}$-begasten Vegetationsperioden 2012 und 2013, entsprechend den Erntezeitpunkt von 1 Tag und 1 Woche (2012) und 1 Jahr nach der ${ }^{14} \mathrm{C}$-Markierung (2013); Daten zeigen MW \pm SE; $n=3-10$, BA = Baumart; Two- Way-ANOVA mit den Faktoren Zeit $(0,2012,2013)$ und Baumart (Ahorn, Buche, Linde)

Bei Buche und Linde wurden neben biometrischen Parametern jeweils Mykorrhizierungs - und Vitalitätsraten sowie beim Ahorn die Kolonisierung der Wurzeln mit Arbuskeln, Vesikeln und Hyphen erhoben (Tabelle 5.1). Bei der Buche wurde eine Mykorrhizierungsrate in den Kontrollen von $77 \pm 13,1 \%$ und in den markierten Pflanzen von 72,5 $\pm 13 \%$ in 2012 und 67,3 $\pm 2,6 \%$ in 2013 beobachtet. Die Mykorrhizierungsrate der Linde betrug 89,7 $\pm 4 \%$ in den Kontrollen und 96,2 \pm 2 $\%$ in 2012 bzw. 89,7 $\pm 4,8 \%$ in 2013. Die Analyse der Ahornwurzeln ergab, dass die Gesamtmykorrhizierung, d.h. Mykorrhizierung mit Hyphen, Arbuskeln und Vesikeln in der Summe, zwischen 19,4 $\pm 1 \%$ und $30 \pm 3,1 \%$ lag. Über den Versuchszeitraum zeigte sich kein signifikanter Unterschied in der Mykorrhizierung innerhalb der 
Baumarten $(p=0,34)$. Es wurde jedoch festgestellt, dass die Mykorrhizierung beim Ahorn deutlich niedriger als bei Buche und Linde war $(p=0)$.

Tabelle 5.1: Mykorrhizierungs- und Vitalitätsraten der Wurzelspitzen an Buche und Linde sowie Kolonisierungsraten der Wurzeln des Ahorns, Daten zeigen MW \pm SE an den Zeitpunkten 0 (Kontrolle), 2012 und 2013; n.b. = nicht bestimmt

\begin{tabular}{|l|l|l|l|l|l|l|}
\hline \multirow{2}{*}{ Baumart } & \multirow{2}{*}{ Zeit } & n & \multicolumn{3}{|l|}{ Myk.rate (\%) } & \multicolumn{2}{l|}{ Vitalitätsrate } \\
$(\%)$
\end{tabular}

5.3.2 Anreicherung von altem und neuem Kohlenstoff in Pflanzen- und Bodenkompartimenten

\subsubsection{1 ${ }^{13} \mathrm{C}$ und ${ }^{14} \mathrm{C}$ in oberirdischen Pflanzenkompartimenten}

Die Begasung mit ${ }^{13} \mathrm{CO}_{2}$ wurde über einen Zeitraum von zwei Vegetationsperioden in den Jahren 2011 und 2012 mit Unterbrechung in der Vegetationsruhe durchgeführt. Die ${ }^{14} \mathrm{CO}_{2}$-Begasung wurde in Form einer 16-stündigen Exposition im Anschluss an die ${ }^{13} \mathrm{CO}_{2}$-Begasung in 2012 durchgeführt. Bei den Ergebnissen ist zu beachten, dass es sich um verschiedene Erntezeitpunkte in Bezug auf die Dauer der ${ }^{13} \mathrm{C}$ - und ${ }^{14} \mathrm{C}$-Exposition handelt. Vom Beginn der ${ }^{13} \mathrm{CO}_{2}$-Begasung bis zum Ende dauerte dieses Experiment insgesamt 737 Tage. Durch die Unterbrechung der ${ }^{13} \mathrm{CO}_{2}$ Begasung in der Vegetationsruhe belief sich die ${ }^{13} \mathrm{CO}_{2}$-Expostion insgesamt auf 216 Tage. Ausgehend vom Ende der ${ }^{13} \mathrm{CO}_{2}$-Begasung vergingen bis zum 1. Erntezeitpunkt nach der ${ }^{14} \mathrm{CO}_{2}$-Begasung 20 Tage, was bedeutet, dass Tag 20 der ${ }^{13} \mathrm{CO}_{2}$-Begasung mit dem 1. Tag der ${ }^{14} \mathrm{CO} 2$-Begasung übereinstimmt; Tag 28 von 
${ }^{13} \mathrm{CO}_{2}$ mit Tag 7 von ${ }^{14} \mathrm{CO}_{2}$ sowie Tag 391 von ${ }^{13} \mathrm{CO}_{2}$ mit Tag 369 (= $1 \mathrm{Jahr}$ ) von ${ }^{14} \mathrm{CO}_{2}$ übereinstimmt.

Die ${ }^{13} \mathrm{C}$ - Anreicherung in den Blättern zeigte bei den drei Baumarten einen ähnlichen Verlauf und eine nahezu identische Höhe (Abbildung 5.3.3 a). Die Kontrollen zeigten eine natürliche Signatur von - $27 \pm 0,3 \partial \%$ im Ahorn, - $27 \pm 0,2 \partial \%$ in der Buche und $-27 \pm 0,5 \partial \%$ in der Linde. Im Verlauf der Vegetationsperiode wurde eine starke Anreicherung von ${ }^{13} \mathrm{C}$ in den Blättern beobachtet. Erst in der folgenden Vegetationsperiode ohne ${ }^{13} \mathrm{CO}_{2}$-Begasung wurde ein Abfall gefunden. Jedoch lag die Anreicherung am Ende noch deutlich über der natürlichen Abundanz $(p<0,01$; Tabelle 5.2) und ist deshalb auf die Einlagerung gespeicherter ${ }^{13} \mathrm{C}$-Verbindungen zurückzuführen. Im Verlauf der Vegetationsperiode zeigten sich signifikante Unterschiede in Bezug auf die Zeitpunkte, jedoch nicht in Bezug auf die Baumarten (Abbildung 5.3.3 a (Mitte)).

Auch die kurzfristige ${ }^{14} \mathrm{CO}_{2}$-Begasung führte zu einer Anreicherung in den Blättern (Abbildung 5.3 .3 b). Im Vergleich zur ${ }^{13} \mathrm{C}$-Anreicherung sank die ${ }^{14} \mathrm{C}$-Anreicherung bei Ahorn und Linde jedoch bereits zum Zeitpunkt nach einer Woche und im folgenden Jahr weiter in allen Baumarten ab, wobei die sie am Ende des Versuches dennoch über der der Kontrollen ( $p<0,01$; Tabelle 5.2) verblieb. Die natürliche Signatur lag beim Ahorn bei $-3 \pm 1 \mathrm{dpm} / \mathrm{g}$, bei der Buche bei $4 \pm 7 \mathrm{dpm} / \mathrm{g}$ und bei der Linde bei $13 \pm 5 \mathrm{dpm} / \mathrm{g}$ lag. 

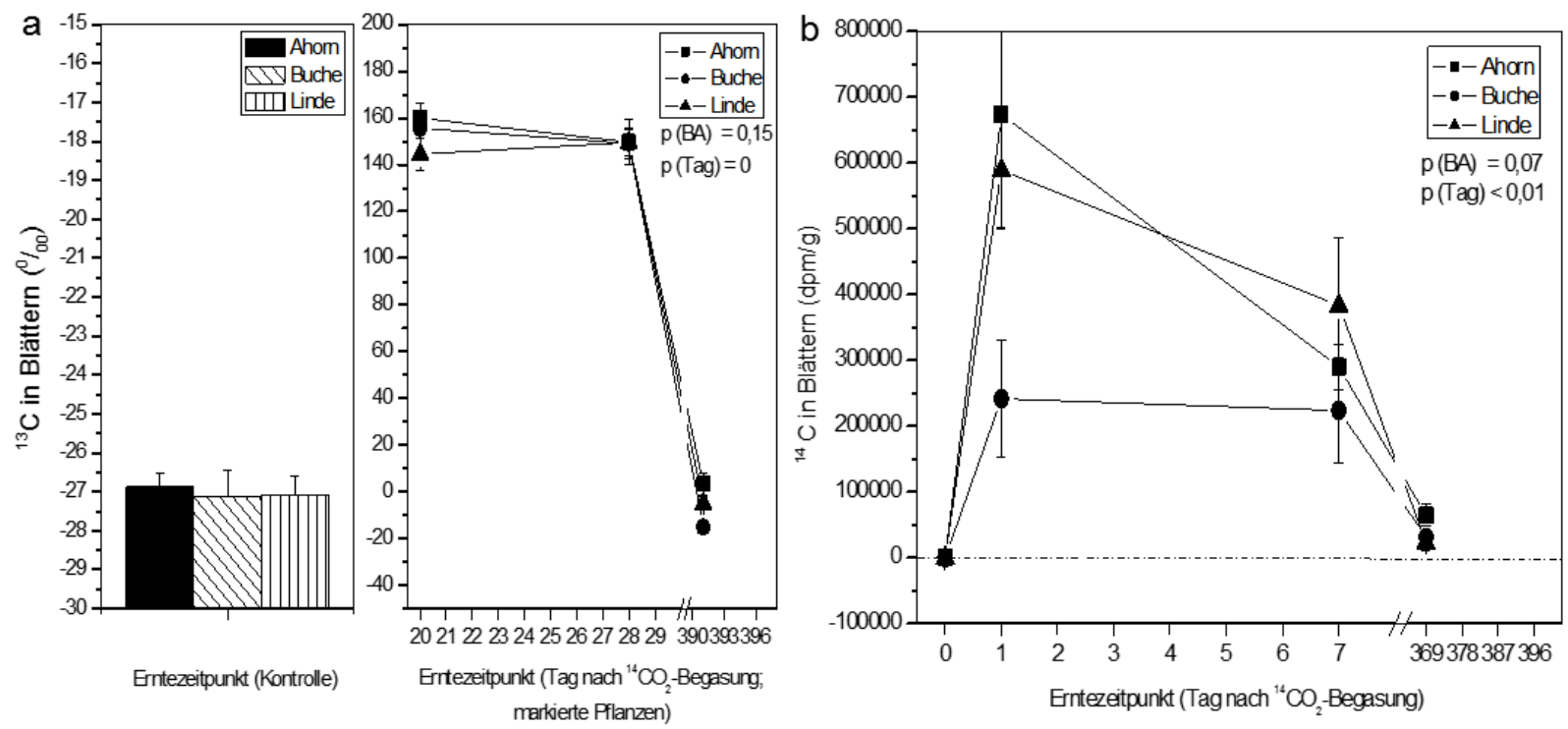

Abbildung 5.3.3: ${ }^{13} \mathrm{C}$ - Anreicherung (a; links und Mitte) in Blättern von Ahorn, Buche und Linde an den Tagen 0, 20, 28 und 391 nach ${ }^{13} \mathrm{CO}_{2}$-Begasung; ${ }^{14} \mathrm{C}$ - Anreicherung in Blättern (b, rechts) von Ahorn, Buche und Linde an den Tagen 0, 1, 7 und 369; entsprechend einem Erntezeitpunkt von 1 Tag, 1 Woche und 1 Jahr nach der ${ }^{14} \mathrm{CO}_{2}$-Begasung (Tag $0=$ Kontrolle); Daten zeigen MW $\pm \mathrm{SE}, \mathrm{n}=3-5$; Two-way-ANOVA mit Faktoren Baumart (BA) und Tag

Die langfristige ${ }^{13} \mathrm{CO}_{2}$-Begasung führte auch zu einer massiven Anreicherung von ${ }^{13}$ C im Spross (Abbildung 5.3.4 a (Mitte)) beobachtet, die dann erst in der folgenden Vegetationsperiode absank. Nach einem Jahr lag die Anreicherung von ${ }^{13} \mathrm{C}$ dennoch über der natürlichen Abundanz ( $p<0,01$, Tabelle 5.2), die beim Ahorn in der Kontrolle bei $-24,9 \pm 0,7 \partial \%$, bei der Buche bei $-26 \pm 0,3 \partial \%$ und bei der Linde bei $-24,8 \pm 0,9 \partial \%$ lag (Abbildung 5.3.4 a (links)).

Die ${ }^{13} \mathrm{C}$-Akkumulation über der natürlichen Abundanz im Spross wies signifikante Unterschiede zwischen den Baumarten auf (Abbildung 5.3.4 a (Mitte)) und betrug beim Ahorn an Tag 20 159,6 \pm 5,1 $\partial \%$, an Tag 28 150,8 $\pm 9 \partial \%$ und nach einem Jahr an Tag $39130,9 \pm 12,2 \partial \%$. Bei der Buche lag die ${ }^{13} \mathrm{C}$-Anreicherung bei 112,4 $\pm 2,6 \%$ bzw. 119,1 $\pm 11,3 \partial \%$ zu Beginn und bei $-2,03 \pm 2,6 \partial \%$ in der folgenden Vegetationsperiode (Abbildung 5.3.4 (Mitte)). Bei der Linde lag die ${ }^{13} \mathrm{C}$-Anreicherung zu Beginn bei 131,7 $\pm 10,4 \partial \%$ bzw. 113,9 $\pm 10,5 \partial \%$ und in der folgenden Vegetationsperiode bei $10,8 \pm 4,1 \partial \%$.

Die überlagerte kurzfristige ${ }^{14} \mathrm{CO}_{2}$-Begasung zeigte ebenfalls eine Anreicherung zu Beginn und einen Abfall in der folgenden Vegetationsperiode, wobei sich die Baumarten auch hier signifikant unterschieden (Abbildung 5.3.4 b). Am 
Versuchsende lag die ${ }^{14} \mathrm{C}$-Akkumulation jedoch noch über der der Kontrollen $(p=0$, Tabelle 5.2).

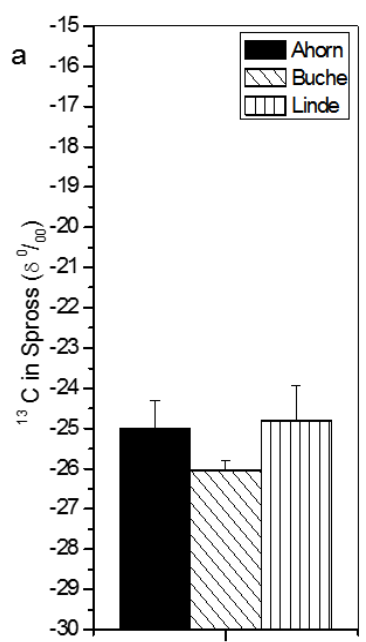

Erntezeitpunkt (Kontrollen)
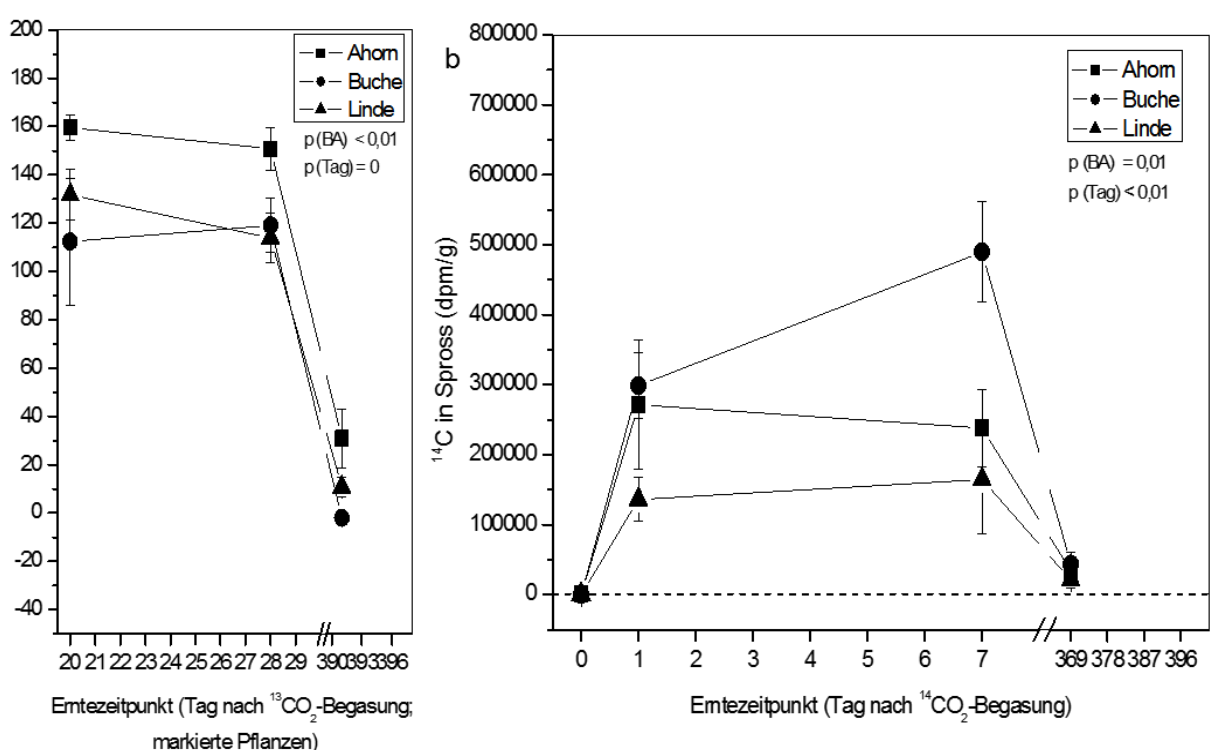

Abbildung 5.3.4: ${ }^{13} \mathrm{C}$ - Akkumulation (a; links) im Spross von Ahorn, Buche und Linde an den Tagen 0 , 20, 28 und 391 nach ${ }^{13} \mathrm{CO}_{2}$-Begasung; ${ }^{14} \mathrm{C}$ - Anreicherung im Spross (b, rechts) von Ahorn, Buche und Linde an den Tagen 0, 1, 7 und 369; entsprechend einem Erntezeitpunkt von 1 Tag, 1 Woche und 1 Jahr nach der ${ }^{14} \mathrm{CO}_{2}$-Begasung (Tag $0=$ Kontrolle); Daten zeigen $\mathrm{MW} \pm \mathrm{SE}, \mathrm{n}=3-5$; Two-way-ANOVA mit Faktoren Baumart (BA) und Tag

\subsubsection{2 ${ }^{13} \mathrm{C}$ und ${ }^{14} \mathrm{C}$ in unterirdischen Pflanzenkompartimenten}

In der unterirdischen Biomasse führte die langfristige ${ }^{13} \mathrm{CO}_{2}$-Begasung ebenfalls $\mathrm{zu}$ einer starken Anreicherung in den Grobwurzeln (Abbildung 5.3.5 a (Mitte), Feinwurzeln (Abbildung 5.3.6 a (Mitte)) und Wurzelspitzen (Abbildung 5.3.7 a (Mitte)), die sich kurzfristig in den einzelnen Organen kaum änderte und erst in der folgenden Vegetationsperiode absank. Ein signifikanter Unterschied zwischen den Baumarten wurde dabei jedoch nur in den Grobwurzeln beobachtet (Abbildung 5.3.5 a (Mitte)). Am Ende des Versuches lag die ${ }^{13} \mathrm{C}$-Anreicherung von Grob- und Feinwurzeln noch signifikant über der natürlichen Signatur ( $p_{G G W}<0,01$, $p_{F W}<0,01$; Tabelle 5.2), wobei zwischen den Kontrollen und den markierten Wurzelspitzen nach einem Jahr kein Unterschied mehr zu beobachten war ( $p w s=0,16$; Tabelle 5.2). Die natürliche Signatur von ${ }^{13} \mathrm{C}$ in den Grobwurzeln lag beim Ahorn bei $-24,6 \pm 0,6 \partial \%$, bei der Buche bei $-25,5 \pm 0,2 \partial \%$ und bei der Linde bei $-23,5 \pm 0,7 \partial \%$ (Abbildung 
5.3.5 a (links)). In den Feinwurzeln betrug sie beim Ahorn bei $-25,4 \pm 0,8 \partial \%$, bei der Buche bei $-26,9 \pm 0,4 \partial \%$ und bei der Linde bei -23,9 $\pm 0,7 \partial \%$ (Abbildung 5.3.6 a (links)) sowie in den Wurzelspitzen beim Ahorn bei $-26,1 \pm 1,1 \partial \%$, bei der Buche bei $-23,2 \pm 0,5 \partial \%$ und bei der Linde bei $-22,6 \pm 0,4 \partial \%$ (Abbildung 5.3.7 a (links)). In Bezug auf Grob-und Feinwurzeln kann daher von einer Einlagerung von langfristigem ${ }^{13} \mathrm{C}$ ausgegangen werden.

Die überlagerte ${ }^{14} \mathrm{CO}_{2}$-Begasung zeigte, dass auch die kurzfristigen C-Ressourcen in Grobwurzeln (Abbildung 5.3.5 b), Feinwurzeln (Abbildung 5.3.6 b) und den Wurzelspitzen (Abbildung 5.3.7 b) gespeichert wurden. Nach einer Anreicherung zu Beginn, verblieb das Niveau in den Grobwurzeln und Feinwurzeln kurzfristig nahezu konstant und stieg bei den Wurzelspitzen sogar noch an. Dabei wurden zwischen den Baumarten jedoch keine signifikanten Unterschiede beobachtet (Abbildung 5.3.7 b).In der folgenden Vegetationsperiode sank die ${ }^{14} \mathrm{C}$-Anreicherung in den drei Kompartimenten $\mathrm{ab}$, verblieb jedoch über den Kontrollen ( $p_{\mathrm{GW}}<0,01$; $\mathrm{p}_{\mathrm{FW}}=0$; $\mathrm{p}_{\mathrm{ws}}<$ 0,01 ; Tabelle 5.2). Daher kann hier ebenfalls von einer Speicherung des kurzfristigen ${ }^{14} \mathrm{C}$ ausgegangen werden.

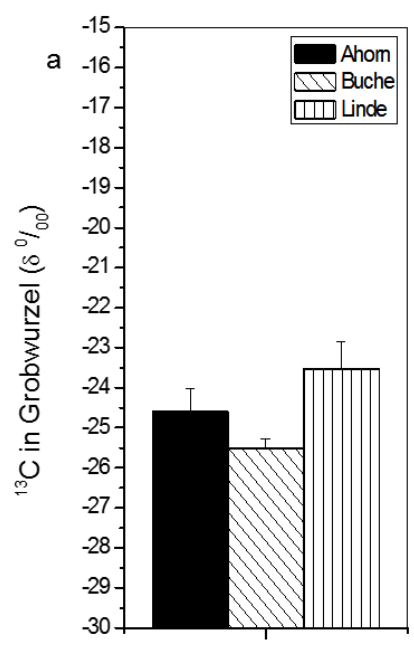

Emtezeitpunkt (Kontrollen)
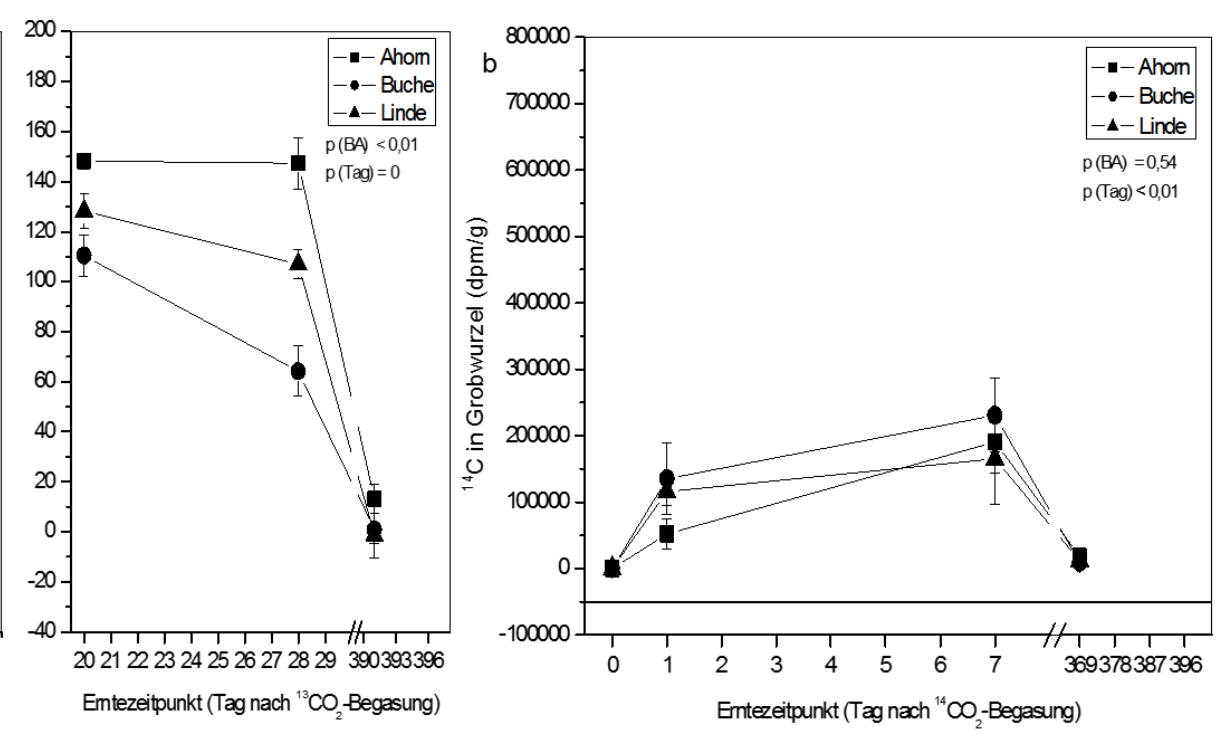

Abbildung 5.3.5: ${ }^{13} \mathrm{C}$ - Anreicherung (a; links) in Grobwurzeln von Ahorn, Buche und Linde an den Tagen 0, 20, 28 und 391 nach ${ }^{13} \mathrm{CO}_{2}$-Begasung; ${ }^{14} \mathrm{C}$ - Anreicherung in Grobwurzeln (b, rechts) von Ahorn, Buche und Linde an den Tagen 0, 1, 7 und 369; entsprechend einem Erntezeitpunkt von 1 Tag, 1 Woche und 1 Jahr nach der ${ }^{14} \mathrm{CO}_{2}$-Begasung (Tag $0=$ Kontrolle); Daten zeigen $\mathrm{MW} \pm \mathrm{SE}, \mathrm{n}=$ 3-5; Two-way-ANOVA mit Faktoren Baumart (BA) und Tag 


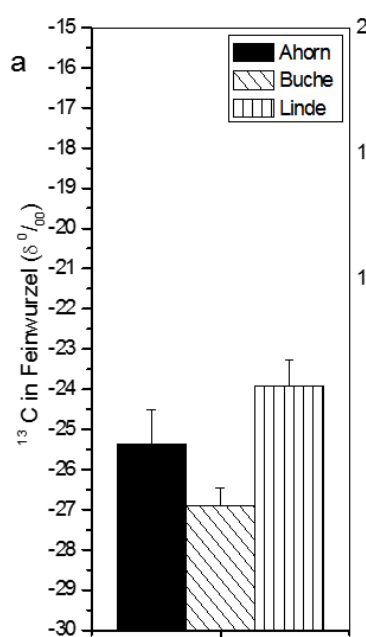

Emtezeitpunkt (Kontrollen)

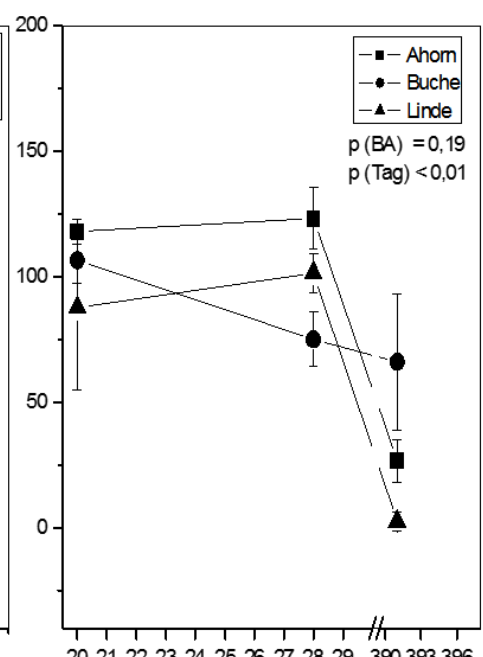

Emtezeitpunkt (Tag nach ${ }^{13} \mathrm{CO}_{2}$-Begasung; markierte Pflanzen)

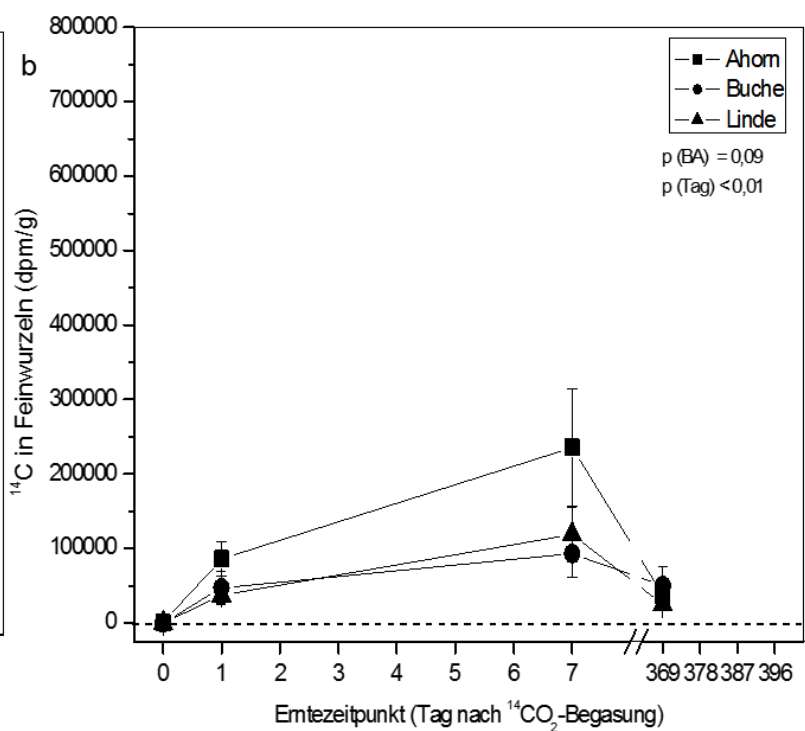

Abbildung 5.3.6: ${ }^{13} \mathrm{C}$ - Anreicherung (a; links) in Feinwurzeln von Ahorn, Buche und Linde an den Tagen 0, 20, 28 und 391 nach ${ }^{13} \mathrm{CO}_{2}$-Begasung; ${ }^{14} \mathrm{C}$ - Anreicherung in Feinwurzeln (b, rechts) von Ahorn, Buche und Linde an den Tagen 0, 1, 7 und 369; entsprechend einem Erntezeitpunkt von 1 Tag, 1 Woche und 1 Jahr nach der ${ }^{14} \mathrm{CO}_{2}$-Begasung (Tag $0=$ Kontrolle); Daten zeigen $\mathrm{MW} \pm \mathrm{SE}, \mathrm{n}=$ 3-5; Two-way-ANOVA mit Faktoren Baumart (BA) und Tag
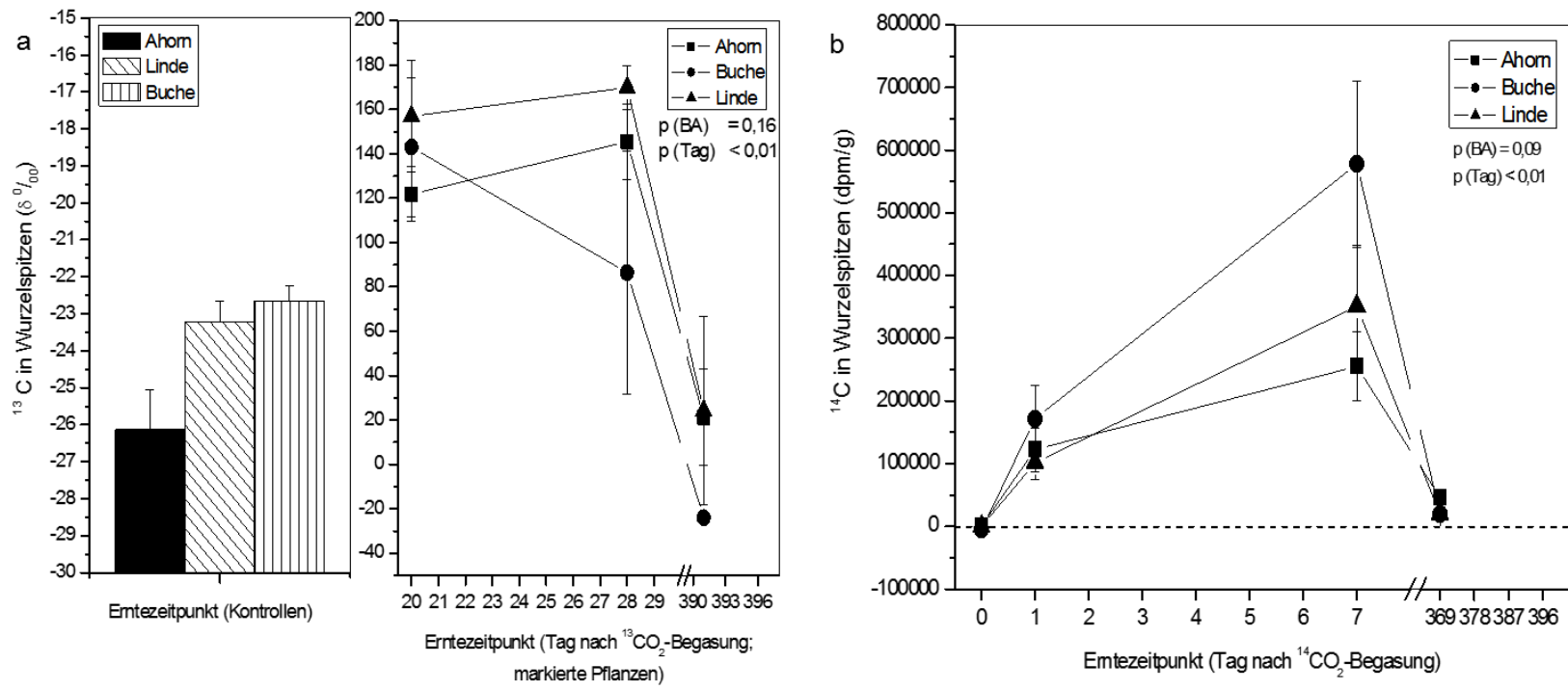

Abbildung 5.3.7: ${ }^{13} \mathrm{C}$ - Anreicherung (a; links) in Wurzelspitzen von Ahorn, Buche und Linde an den Tagen 0, 20, 28 und 391 nach ${ }^{13} \mathrm{CO}_{2}$-Begasung; ${ }^{14} \mathrm{C}$ - Anreicherung in Wurzelspitzen (b, rechts) von Ahorn, Buche und Linde an den Tagen 0, 1, 7 und 369; entsprechend einem Erntezeitpunkt von 1 Tag, 1 Woche und 1 Jahr nach der ${ }^{14} \mathrm{CO}_{2}$-Begasung (Tag $0=$ Kontrolle); Daten zeigen $\mathrm{MW} \pm \mathrm{SE}, \mathrm{n}=$ 3-5; Two-way-ANOVA mit Faktoren Baumart (BA) und Tag 


\subsubsection{3 ${ }^{13} \mathrm{C}$ und ${ }^{14} \mathrm{C}$ in Rhizosphärenboden und Mischboden}

Die langfristige ${ }^{13} \mathrm{CO}_{2}$-Begasung führte auch im Rhizosphären- und Mischboden zu einer Anreicherung von ${ }^{13} \mathrm{C}$ (Abbildung 5.3.8 a (Mitte), Abbildung 5.3.9 a (Mitte)). Die Anreicherung dabei war jedoch geringer als in den oberirdischen (vgl. Abbildungen in 5.3.2.1) und unterirdischen Pflanzenkompartimenten (vgl. Abbildungen in 5.2.2.2). Die Baumarten unterschieden sich jedoch nicht in der Höhe der Anreicherung (Abbildung 5.3.8 a (Mitte)). Nach der deutlichen Anreicherung von ${ }^{13} \mathrm{C}$ im Rhizosphärenboden zu Beginn sank diese jedoch bereits nach kurzer Zeit über den Versuchsverlauf zum Ende hin ab. Es verblieb aber dennoch eine signifikante Anreicherung über der natürlichen Abundanz auch nach einem Jahr $(p=0$; Tabelle 5.2). Die natürliche Abundanz von ${ }^{13} \mathrm{C}$ im Rhizosphärenboden lag beim Ahorn bei 25,7 \pm 0,2 $\partial \%$, bei der Buche bei $-26,3 \pm 0,1 \partial \%$ und bei der Linde bei $-26,2 \pm$ $0,1 \partial \%$ (Abbildung 5.3.8 a).

Auch im Mischboden (bulk soil) kam es nach der langfristigen ${ }^{13} \mathrm{CO}_{2}$-Begasung zu einer Anreicherung von ${ }^{13} \mathrm{C}$ (Abbildung 5.3.9 a (Mitte)). Die Anreicherung verblieb über den gesamten Versuchszeitraum relativ konstant ohne deutliche Unterschiede zwischen den Baumarten (Abbildung 5.3.9 a (Mitte)) und lag am Ende des Versuches noch über der natürlichen Abundanz ( $p<0,01$, Tabelle 5.2). Die natürliche Abundanz von ${ }^{13} \mathrm{C}$ im Mischboden lag beim Ahorn bei - 25,8 \pm 0,2 $\partial \%$, bei der Buche bei 26,2 \pm 0,1 $\partial \%$ und bei der Linde bei - 26,2 \pm 0,2 $\partial \%$ (Abbildung 5.3.9 a (links)).

Daher kann im Rhizosphärenboden von einer Speicherung des langfristigen C ausgegangen werden.

Die überlagerte ${ }^{14} \mathrm{CO}_{2}$-Pulsmarkierung zeigte, dass die kurzfristigen C-Ressourcen ebenfalls gespeichert wurden, da die Anreicherung am Ende des Versuches sowohl im Rhizosphärenboden als auch im Mischboden noch über den Kontrollen lag ( $p=0$ bzw. p < 0,01; Tabelle 5.2).

In Bezug auf die ${ }^{14} \mathrm{C}$-Anreicherung, die sich in beiden Kompartimenten kurzfristig kaum änderte und in der folgenden Vegetationsperiode absank (Abbildung 5.3.8 b, 5.3.9 b), zeigten sich beim Rhizosphärenboden leicht signifikante Unterschiede zwischen den Baumarten $(p=0,05)$ und beim Mischboden deutliche Unterschiede ( $p$ $=0,02)$. In beiden Kompartimenten war die Anreicherung im Ahorn am höchsten. 

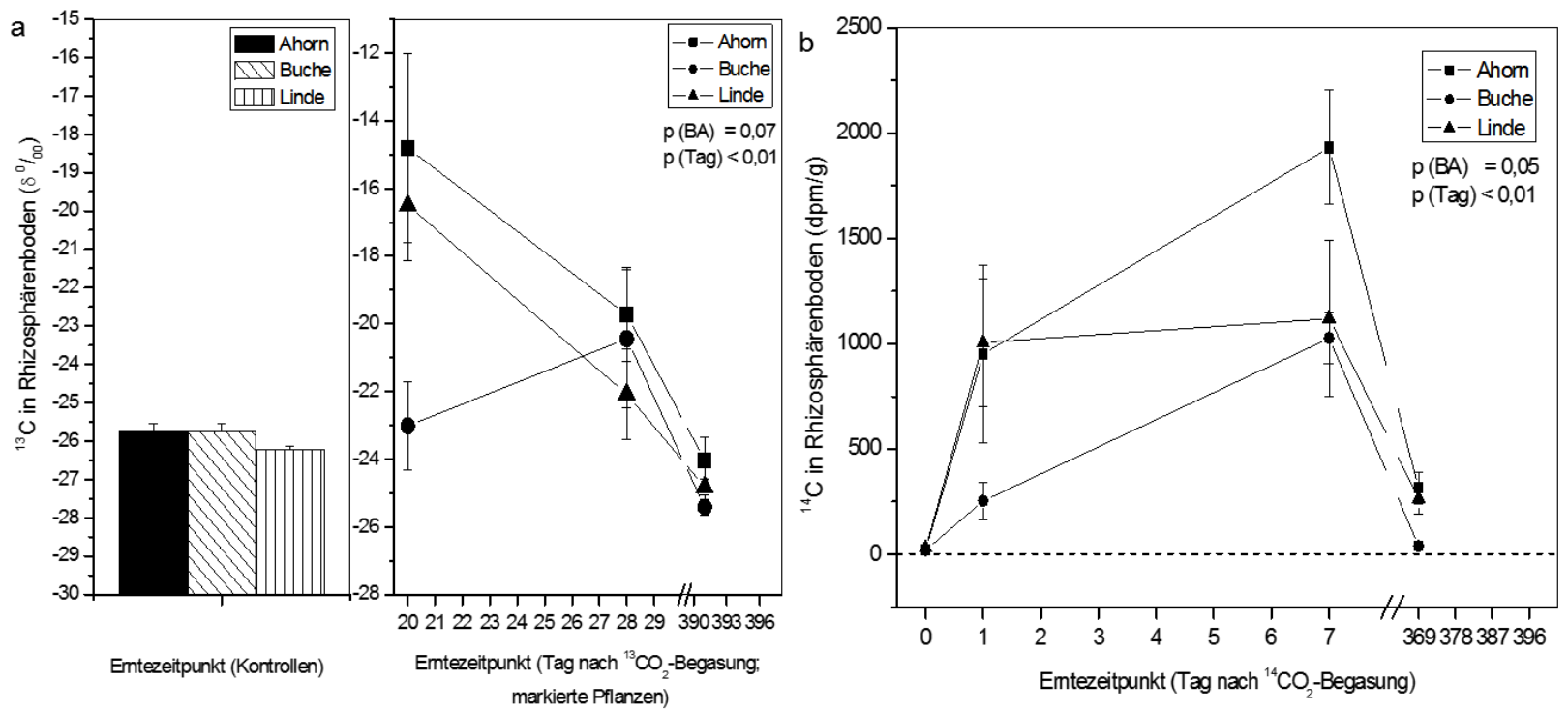

Abbildung 5.3.8: ${ }^{13} \mathrm{C}$ - Anreicherung (a; links) im Rhizoshärenboden von Ahorn, Buche und Linde an den Tagen 0, 20, 28 und 391 nach ${ }^{13} \mathrm{CO}_{2}$-Begasung; ${ }^{14} \mathrm{C}$ - Anreicherung im Rhizoshärenboden (b, rechts) von Ahorn, Buche und Linde an den Tagen 0, 1, 7 und 369; entsprechend einem Erntezeitpunkt von $1 \mathrm{Tag}, 1$ Woche und $1 \mathrm{Jahr}$ nach der ${ }^{14} \mathrm{CO}_{2}$-Begasung (Tag $0=$ Kontrolle); Daten zeigen MW $\pm \mathrm{SE}, \mathrm{n}=3-5$; Two-way-ANOVA mit Faktoren Baumart (BA) und Tag

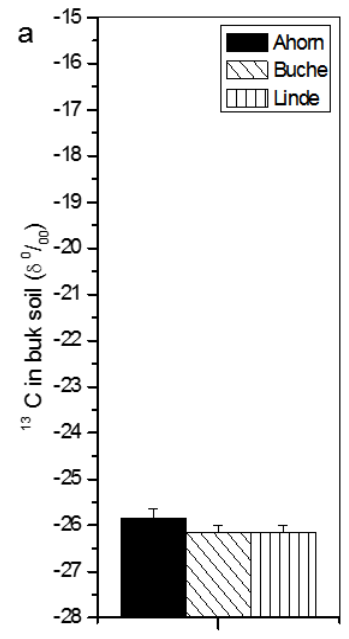

Emtezeitpunkt ( Kontrollen)

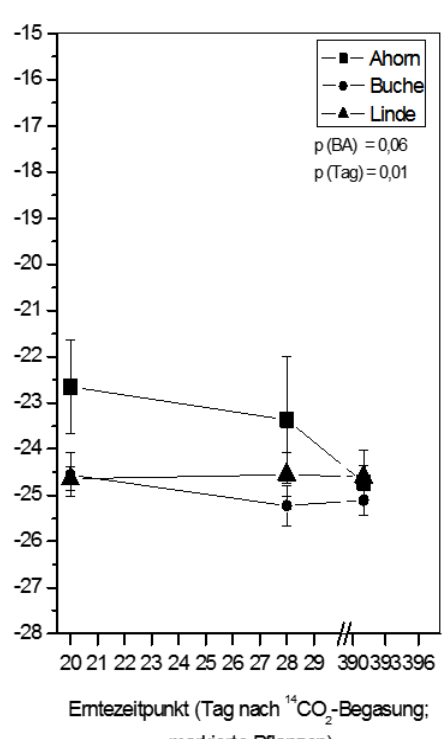

markierte Pflanzen)

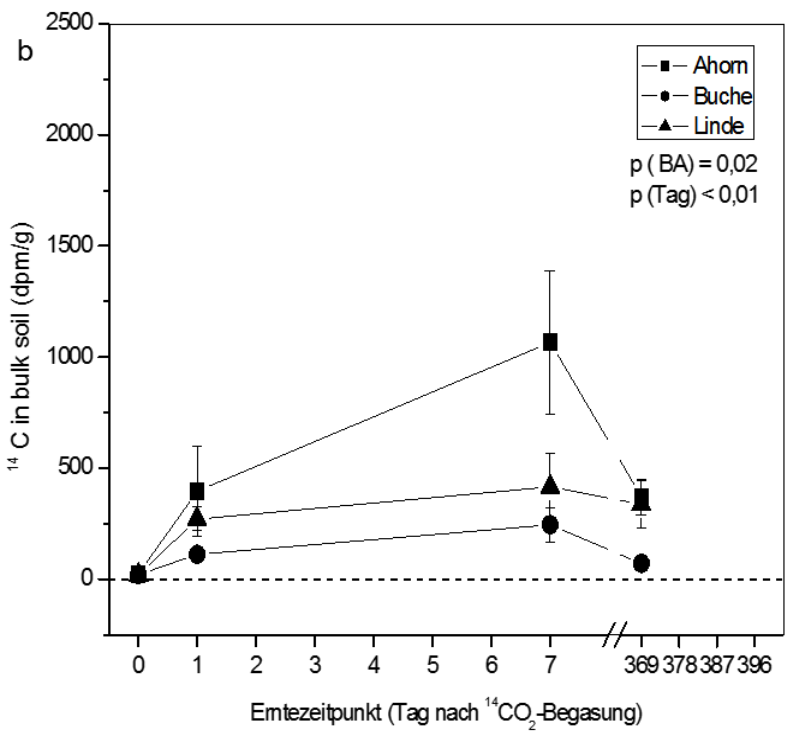

Abbildung 5.3.9: ${ }^{13} \mathrm{C}$ - Anreicherung (a; links) im Mischboden (bulk soil) von Ahorn, Buche und Linde an den Tagen 0, 20, 28 und 391 nach ${ }^{13} \mathrm{CO}_{2}$-Begasung; ${ }^{14} \mathrm{C}$ - Anreicherung im Mischboden (bulk soil) (b, rechts) von Ahorn, Buche und Linde an den Tagen 0, 1, 7 und 369; entsprechend einem Erntezeitpunkt von $1 \mathrm{Tag}, 1$ Woche und $1 \mathrm{Jahr}$ nach der ${ }^{14} \mathrm{CO}_{2}$-Begasung (Tag $0=$ Kontrolle); Daten zeigen MW $\pm S E, n=3-5$; Two-way-ANOVA mit Faktoren Baumart (BA) und Tag 
Tabelle 5.2: Two-Way-ANOVA: Kontrolle gegen Messwerte nach 1 Jahr; Daten zeigen MW \pm SE; Faktor 1: BA (Baumart), Faktor 2: Zeitpunkt (1 Jahr); p-Werte beziehen sich jeweils auf ein Kompartiment mit beiden Faktoren BA und Zeit

\begin{tabular}{|c|c|c|c|c|c|c|c|c|c|c|c|c|c|}
\hline \multirow[t]{3}{*}{ Baumart } & \multirow[t]{3}{*}{ Kompartiment } & \multicolumn{2}{|l|}{${ }^{13} \mathrm{C}($ Delta $\% / 00)$} & \multicolumn{2}{|c|}{${ }^{13} \mathrm{C}($ Delta $\% 00)$} & \multicolumn{2}{|c|}{${ }^{13} \mathrm{C}($ Delta $\% 00)$} & \multirow{2}{*}{\multicolumn{2}{|c|}{$\begin{array}{l}{ }^{14} \mathrm{C}(\mathrm{dpm} / \mathrm{g}) \\
\text { Kontrolle }\end{array}$}} & \multirow{3}{*}{$\begin{array}{l}{ }^{14} \mathrm{C}(\mathrm{dpm} / \mathrm{g}) \\
1 \mathrm{Jahr} \\
\mathrm{MW}\end{array}$} & \multirow[b]{3}{*}{ SE } & \multicolumn{2}{|c|}{${ }^{14} \mathrm{C}(\mathrm{dpm} / \mathrm{g})$} \\
\hline & & \multirow{2}{*}{$\begin{array}{l}\text { Kontrolle } \\
\text { MW }\end{array}$} & \multirow[b]{2}{*}{ SE } & \multirow{2}{*}{$\begin{array}{l}1 \text { Jahr } \\
\text { MW }\end{array}$} & \multirow[b]{2}{*}{ SE } & \multirow[b]{2}{*}{$p(B A)$} & \multirow[b]{2}{*}{$\mathrm{p}$ (Zeit) } & & & & & & \\
\hline & & & & & & & & MW & SE & & & $p(B A)$ & $\mathrm{p}$ (Zeit) \\
\hline Ahorn & Blätter & $-26,876$ & 0,34 & 26,740 & 25,96 & 0,05 & $<0,01$ & $-3,46$ & 1,02 & 114229,39 & 56938,13 & 0,12 & $<0,01$ \\
\hline Buche & Blätter & $-27,131$ & 0,70 & $-13,742$ & 0,56 & & & 3,81 & 6,79 & 25976,67 & 4146,16 & & \\
\hline Linde & Blätter & $-27,075$ & 0,48 & $-5,177$ & 3,49 & & & 13,15 & 4,78 & 23002,89 & 12201,85 & & \\
\hline Ahorn & Spross & $-24,994$ & 0,70 & 30,903 & 12,24 & 0,09 & $<0,01$ & 17,40 & 13,82 & 39263,99 & 7746,74 & 0,33 & 0 \\
\hline Buche & Spross & $-26,034$ & 0,25 & $-2,037$ & 2,62 & & & 1,39 & 2,24 & 43555,16 & 16002,31 & & \\
\hline Linde & Spross & $-24,023$ & 0,66 & 9,032 & 3,08 & & & 1,78 & 0,01 & 20646,40 & 14787,61 & & \\
\hline Ahorn & Grobwurzel & $-24,595$ & 0,58 & 13,176 & 5,91 & 0,32 & $<0,01$ & 31,21 & 11,48 & 17986,45 & 4354,59 & 0,27 & $<0,01$ \\
\hline Buche & Grobwurzel & $-25,507$ & 0,23 & 0,842 & 11,21 & & & 6,08 & 4,61 & 9146,24 & 2594,18 & & \\
\hline Linde & Grobwurzel & $-23,530$ & 0,69 & $-1,273$ & 3,20 & & & 8,89 & 2,26 & 10649,12 & 2783,56 & & \\
\hline Ahorn & Feinwurzel & $-25,358$ & 0,84 & 26,697 & 8,57 & 0,09 & $<0,01$ & 119,39 & 16,18 & 36990,21 & 12151,67 & 0,51 & 0 \\
\hline Buche & Feinwurzel & $-26,907$ & 0,44 & 66,009 & 27,28 & & & 2,76 & 0,01 & 50162,89 & 25990,69 & & \\
\hline Linde & Feinwurzel & $-23,932$ & 0,65 & 4,184 & 4,33 & & & 5,56 & 1,98 & 24646,65 & 6763,38 & & \\
\hline Ahorn & Wurzelspitzen & $-26,131$ & 1,08 & 21,172 & 21,80 & 0,62 & 0,16 & 10,57 & 9,16 & 765,41 & 151,13 & 0,01 & $<0,01$ \\
\hline Buche & Wurzelspitzen & $-23,213$ & 0,54 & $-24,007$ & 1,30 & & & $-88,75$ & 35,48 & 328,03 & 81,78 & & \\
\hline Linde & Wurzelspitzen & $-22,647$ & 0,39 & 24,224 & 42,51 & & & $-8,76$ & 2,72 & 330,68 & 49,30 & & \\
\hline Ahorn & Rhizoboden & $-25,761$ & 0,21 & $-24,039$ & 1,52 & 0,08 & 0 & 22,19 & 3,87 & 315,49 & 170,36 & 0,1 & 0 \\
\hline Buche & Rhizoboden & $-26,303$ & 0,12 & $-25,424$ & 0,24 & & & 20,07 & 0,88 & 40,03 & 20,98 & & \\
\hline Linde & Rhizoboden & $-26,242$ & 0,11 & $-24,831$ & 0,24 & & & 30,94 & 6,96 & 264,80 & 75,71 & & \\
\hline Ahorn & Mischboden & $-25,843$ & 0,20 & $-24,724$ & 0,37 & 0,66 & $<0,01$ & 24,31 & 6,24 & 370,20 & 80,13 & 0,12 & $<0,01$ \\
\hline Buche & Mischboden & $-26,150$ & 0,14 & $-25,106$ & 0,32 & & & 20,82 & 1,73 & 70,11 & 12,67 & & \\
\hline Linde & Mischboden & $-26,153$ & 0,15 & $-24,604$ & 0,57 & & & 18,99 & 0,82 & 337,46 & 108,70 & & \\
\hline
\end{tabular}




\subsection{Diskussion}

In der vorliegenden Untersuchung wurde der Transport von langfristigem und kurzfristigem C sowie deren Verweildauern von den Blättern in die Rhizosphäre von den drei Laubbaumarten Ahorn (Acer pseudo-platanus), Buche (Fagus sylvatica) und Linde (Tilia cordata) analysiert.

Sowohl das langfristige ${ }^{13} \mathrm{C}$ als auch das kurzfristige ${ }^{14} \mathrm{C}$ wurde in der vorliegenden Untersuchung in der ersten Vegetationsperiode in oberirdischen und unterirdischen Pflanzenorganen gespeichert und sank in der folgenden Vegetationsperiode in beiden Fällen auf ein Niveau über der natürlichen Abundanz ab. Dies zeigte zum einen die Speicherung von $\mathrm{C}$ in den einzelnen Kompartimenten und zum anderen den Transport assimiliertem Kohlenstoff zur Rhizosphäre. Da bereits am ersten Messzeitpunkt sowohl ${ }^{13} \mathrm{C}$ als auch ${ }^{14} \mathrm{C}$ in allen Kompartimenten messbar war, muss ein Stofftransport stattgefunden haben. Neben dem Transport von C von den Blättern spielt die C-Speicherung in Pflanzenorganen eine wichtige Rolle als C-sink (Lacointe et al., 2004) und es wurde hier davon ausgegangen, dass C im Verlauf der folgenden Vegetationsperiode verbraucht wurde. Eine deutliche Speicherung von 43 $-75 \%$ von ${ }^{14} \mathrm{C}$ in der oberirdischen Biomasse wurde auch in einem pulse labellingExperiment an Kiefer, Fichte und Birke beobachtet (Pumpanen et al. 2009). Im Verlauf der folgenden Vegetationsperiode sank in der oberirdischen Biomasse die Anreicherung der Isotopen. Keel et al. (2007) stellten bei einer ${ }^{13} \mathrm{CO}_{2}$-Markierung ebenfalls fest, dass 4 Monate nach der Markierung im Spross von Linde und Ahorn kaum noch Anreicherung $\mathrm{zu}$ finden war, was mit unseren Ergebnissen korrespondiert, die Anreicherung in der Buche jedoch anstieg. Keel et al. (2007) interpretieren das sinkende Signal in der Linde als eine niedrige Vermischung von neuem und altem $\mathrm{C}$, wohingegen der Anstieg in Buche und Eiche als starke Vermischung verstanden wird. Aus dieser Sicht kann der ähnliche Verlauf von langund kurzfristigem $\mathrm{C}$ in der vorliegenden Untersuchung ebenfalls als Resultat der Vermischung interpretiert werden.

In der vorliegenden Untersuchung wurde im Gegensatz zu Simard et al. (1997) kein unterschiedliches Verhalten der beiden Isotope beobachtet, die Isotopen verhielten sich ähnlich. In den Grob- und Feinwurzeln verlief die Anreicherung von lang- und kurzfristigem $\mathrm{C}$ ähnlich, wobei in den Wurzelspitzen die Anreicherung von ${ }^{14} \mathrm{C}$ nach einer Woche nach der Markierung sogar nochmal anstieg. 
Es wurde eine Verweilzeit des langfristigem $C$ von mindestens drei Wochen und dem kurzfristigem $\mathrm{C}$ von mindestens einer Woche beobachtet, was die Beobachtungen von Högberg et al. (2008), in der die Verweilzeit von rund zwei Tagen von markiertem C im Spross von Kiefer betrug, deutlich überstieg.

Neben der Speicherung von langfristigem ${ }^{13} \mathrm{C}$ und kurzfristigem ${ }^{14} \mathrm{C}$ in der oberirdischen Biomasse, die bei beiden Isotopen ähnlich war, erfolgte ein Transport von Kohlenstoff in die Rhizosphäre mit einer resultierenden hohen Anreicherung in Grob- und Feinwurzeln sowie den Wurzelspitzen. Die hohe Anreicherung von $C$ in den Wurzeln stimmt mit den Beobachtungen von Pumpanen et al. (2009) überein, nach denen 13-21\% des kurzfristig assimiliertem $C$ in der Wurzel messbar waren und zum Wurzelwachstum genutzt wurden. Dem gegenüber steht die Ansicht von Trueman \& Gonzalez-Meler (2005), dass gespeichertes C nicht für die Wurzelproduktion in Laubbäumen verwendet wird. Die Tatsache, dass in unserer die Anreicherung im Boden deutlich geringer als in den Wurzeln war, deutet allerdings daraufhin, dass $C$ verbraucht wurde. Es lässt sich jedoch nicht sicher sagen, ob dies zwangsläufig die Produktion neuer Wurzeln sein muss. Die Anreicherung im Boden war geringer als in den Pflanzenkompartimenten. Dies kann als starker sink der Wurzeln für Kohlenstoff verstanden werden und die Tatsache, dass die Anreicherung beim endomykorrhizierten Ahorn tendenziell höher als bei der ektomykorrhizierten Buche und Linde war, würde die These stützen, dass die Ektomykorrhiza einen stärkeren Kohlenstoffsink darstellt als die Endomykorrhiza (Finlay \& Soderström, 1992; Marschner 1995).

Unsere Ergebnisse gehen insgesamt konform mit den Beobachtungen von Lynch et al. 2013), die in ihrem Markierungsexperiment an amerikanischen Amberbäumen (Liquidambar styraciflua) festgestellt haben, dass die Wurzeln sowohl aus kurzfristigen als auch längerfristigen Kohlenstoffspeichern gespeist werden. Auf Grund der Speicherung und des Transportes von lang- und kurzfristigem $C$ muss davon ausgegangen werden, dass beide Pools für die Prozesse in der Pflanze gleichgewichtige Bedeutung besitzen. Dies geht mit den Ergebnissen von Bader et al. (2009) konform, in deren Untersuchung die Feinwurzeln eines mit $\mathrm{CO}_{2}$-markierten Mischwaldes sogar nach 6 Jahren zu 51\% aus neuem Kohlenstoff bestanden. 


\subsection{Literatur}

Andrasko K (1990) Global warming and forests. An overview of current knowledge. Unasylva 41: $3-11$

Bader M, Hiltbrunner E, Körner C (2009) Fine root responses of mature deciduous forest trees to free air carbon dioxide enrichment (FACE)

Dykmans J (2013): Stabile Isotope in der terrestrischen Ökologie - Einführung (Tutorial, pdf abgerufen am 14.04.2015 unter http://www.unigoettingen.de/de/123620.html)

Finlay R, Soderström B (1992) Mycorrhiza and carbon flow to the soil. In Allen MF: Mycorrhizal functioning- an integrative plant- functional process. Chapman an dHall, New York

Fitter AH, Graves JD, Watkins NK, Robinson D, Scimgeour C (1998) Carbon transfer between plants and its control in networks of arbuscular mycorrhizas. Functional Ecology 12:406 - 412

Geilenkirchen IT (1911) Eigenschaften, Aufbereitung und Bewegung der Luft für den Verbrennungsprozess. Grundzüge des Eisenhüttenwesens. Springer Berlin Heidelberg 188 - 196

Ghirardo A, Gutknecht J, Zimmer I, Brüggemann N, Schnitzler JP (2011) Biogenic volatile organic compound and respiratory $\mathrm{CO}_{2}$ emisssions after ${ }^{13} \mathrm{C}$.labeling: Online tracing of $\mathrm{C}$ translocation dynamics in poplar plants. PLoS One 6. E17393

Houghton RA (1990) The global effects of tropical deforestation. Environ Sci Tech $24: 414-422$

Högberg P, Nordgren A, Buchmann N,Taylor AFS,Ekblad A, Högberg NM, Nyberg G, Ottosson- Löfvenius M, Read DJ (2001) Large-scale forest girdling shows thta current photosynthesis drives soil respiration. Nature 411: $789-792$

Högberg $\mathrm{P}$, Högberg $\mathrm{MN}$, et al. (High temporal resolution tracing of photosynthate carbon from the tree canopy to forest soil microorganisms. New Phytol 177:220 - 228

Keel SG, Siegwolf RTW, Jäggi M, Körner C (2007) Rapid mixing between old and new $C$ pools in the canopy of mature forest trees. Plant Cell Environ 30: 963 - 972

Lacointe A, Deleens E, Ameglio B, Saint-Joanis C, Lelarge M, Vandame GC, Daudet FA (2004) Testing the branch autonomy theory. A $13 \mathrm{C} / 14 \mathrm{C}$ double-labelling experiment on differentially shaded branches. Plant Cell Environ. 27:1159 -1168

Lynch DJ, Matamala R, Iversen CM, Norby RJ, Gonzalez-Meler MA (2013) Stored carbon partly fuels fine-root respiration but is not used for production of new fine roots. New Phytol 199:420 - 430

Marschner H (1995) Mineral nutrition of higher plants. Academic Press, London

Melin E, Nilsson $\mathrm{H}$ (1957) Transport of ${ }^{14} \mathrm{C}$ labelled photosynthate to the fungal associate of pine mycorrhiza. Svenske Botanisk Tidskrift 51:166 - 186

Norby RJ, Ledford J, Reilly CD, Miller NE, O`Neill EG (2004) Fine-root production dominates response of a deciduous forest to atmospheric $\mathrm{CO} 2$ enrichment. PNAS $101: 9686-9693$ 
Pumpanen JS, Heinonsalo J, Rosilo T, Hume KR, Ilvesniemi H (2009) Carbon balance and allocation of assimilated $\mathrm{CO}_{2}$ in Scots pine, Norway spruce and silver birch seedlings determined with gas exchange measurements and ${ }^{14} \mathrm{C}$ pulse labelling. Trees 23:611 - 621

Schneider A, Schmitz K (1989) Seasonal course of translocation and distribution of ${ }^{14} \mathrm{C}$ - labelled photoassimilate in young trees of Larix decidua Mill. Trees 4:185 - 191

Schuur EAG \&Trumbore SE (2006) Partitioning sources of soil respiration in boreal black spruce forest using radiocarbon. Global Change Biology 12:165- 176

Shiroya T, Lister GR, Krotkov G, Nelson D, Slankis V (1962) Translocation oft he products of photosynthesis to roots of pine seedlings. Can J Bot 40: 1125- 1135

Simard SW, Perry DA, Jones MD, Myrold DD, Durall DM, Molina R (1997) Net transfer of carbon between ectomycorrhizal tree species in the field. Nature 388:579 582

Söderström B, Read DJ (1987) Respiratory activity of intact and excised ectomycorrhizal mycelial systems growing in unsterilized soil. Soil Biol Biochem 19:231 - 236

Trueman RJ \& Gonzalez-Meler MA (2005) Accelerated belowground C cycling in a managed agriforest ecosystem exposed to elevated carbon dioxide concentrations. Global Change Biology 11: 1258-1271 


\section{Synopsis}

Die vorliegende Arbeit befasst sich mit dem übergeordneten Thema des Stofftransportes in Pflanzen und ihrer Rhizosphäre. Die Betrachtung verschiedener einheimischer Laubbaumarten nimmt dabei Bezug auf das aktuelle Themengebiet der Biodiversitätsforschung. Methodisch wurden Isotope von $\mathrm{C}$ und $\mathrm{N}$ als Tracer angewandt, um die Stoffflüsse dieser wichtigen Pflanzenelemente zu analysieren.

Die zentrale Baumart in dieser Arbeit war die Buche (Fagus sylvatica), die in Mitteleuropa als dominante und konkurrenzstärkste Baumart bezeichnet wird (Ellenberg, 1996; Röhrig \& Bartsch, 1992). Auf Grund ihrer breiten Standortamplitude tritt sie in Deutschland zum einen in Reinbeständen als auch in Mischbeständen auf. In dieser Arbeit wurde ein Fokus auf sie und weitere einheimische Laubbaumarten gesetzt, um die Einblicke in biodiverse Strukturen in Bezug auf Stoffflüsse in Bäumen $\mathrm{zu}$ vertiefen und die verschiedenen Baumarten miteinander zu vergleichen. Daher wurde die Buche mit der Esche (Fraxinus excelsior) verglichen, die eine Baumart darstellt, die in Buchen-Eschen-Wäldern auftritt (Stinglwagner et al., 2005). Der Vergleich dieser beiden Baumarten war in der vorliegenden Arbeit von Interesse, da Buche und Esche über verschiedene Mykorrhizaformen verfügen. Die Buche ist obligat mit Ektomykorrhizen vergesellschaftet, während die Esche mit arbuskulären Mykorrhizen assoziiert ist (Lang et al., 2011). Der Mykorrhiza wird eine wichtige Bedeutung im Nährstofftransport in den temperaten Breiten zugesprochen (Hobbie \& Hobbie, 2006). Es wird davon ausgegangen, dass in Bezug auf den Kohlenstoff die Ektomykorrhiza eine stärkere Senke ist als die arbuskuläre Mykorrhiza (Finlay \& Soderström 1992; Marschner 1995). In der vorliegenden Arbeit wurde das nicht bestätigt, sondern beobachtet, dass die arbuskulär mykorrhizierte Esche eine größere Senke sowohl für den Kohlenstoff als auch den Stickstoff darstellte.

Baumarten beeinflussen den Nährstoffkreislauf in unterschiedlicher Weise (Finzi et al., 1998, Lovett \& Rueth, 1999) und der Aspekt der Biodiversität wurde in der vorliegenden Untersuchung durch zwei weitere Baumarten, Bergahorn (Acer pseudo-platanus) und Winterlinde (Tilia cordata) vertieft. Beide Baumarten kommen in Mitteleuropa und Deutschland in Vergesellschaftung mit Buche und Esche vor. Die beiden Baumarten wurden ausgewählt, da sie durch ihre Mykorrhizaform die beiden anderen Baumarten ergänzen und den Einblick in Bezug auf die Bedeutung der Mykorrhiza im Nährstofftransfer vertiefen. Die Winterlinde ist wie die Buche mit 
Ektomykorrhizen vergellschaftet, während der Bergahorn mit arbuskulären Mykorrhizen assoziiert ist (Lang et al., 2011).

Neben Kohlenstoff wurde in der vorliegenden Arbeit Stickstoff als weiterer wichtiger Pflanzennährstoff bezüglich des Transportes, in die verschiedenen Pflanzenorgane und die Rhizosphäre betrachtet.

Die Experimente der vorliegenden Arbeit zeigten alle einen Stofftransport innerhalb der Pflanzen. Ausgehend von einem Bodenmarkierungsexperiment in Buchenreinund Mischbeständen wurde die ${ }^{15} \mathrm{~N}$-Aufnahme der Feinwurzeln über den Boden analysiert. Dabei zeigte sich sowohl im Buchenreinbestand als auch im Buchenmischbestand eine starke Anreicherung von ${ }^{15} \mathrm{~N}$ in den Feinwurzeln zu Beginn mit verbleibendem konstanten Niveau über eine Woche. Zudem zeigte der Reinbestand eine höhere Stickstoffanreicherung in den Feinwurzeln als der Mischbestand. Die Translokation von $\mathrm{C}$ und $\mathrm{N}$ von den Blättern in die Rhizosphäre wurde im Folgenden an weiteren Experimenten untersucht. In einer Freilanduntersuchung wurde die unterschiedliche Strategie der Baumarten in Bezug auf $\mathrm{C}$ - und $\mathrm{N}$ - Akkumulation in ektomykorrhizierter Buche und endomykorrhizierter Esche aufgezeigt. Die Bäume wurden dabei über die Blätter mit ${ }^{13} \mathrm{CO}_{2}$ und ${ }^{15} \mathrm{~N}$ markiert und der Transport über einen Zeitraum von 60 Tagen analysiert. Es zeigte sich, dass die endomykorrhizierte Esche sowohl mehr $\mathrm{C}$ als auch $\mathrm{N}$ in ihren Feinwurzeln und Wurzelspitzen anreicherte als die ektomykorrhizierte Buche.

Der Stofftransport von der Pflanze in Richtung Rhizosphäre steht aus physiologischer Sicht im Zusammenhang mit dem Vitalitätszustand der Feinwurzeln. Daher wurde an den beiden untersuchten Baumarten die Methode der visuellen Ansprache von vitalen Wurzeln überprüft. Dabei wurden Wurzelspitzen an Hand von TTC-Tests auf ihren Vitalitätszustand hin überprüft. Aus dem Vergleich von vitalen und trockenen Wurzelspitzen ergab sich, dass die Methode der visuellen Ansprache bei der ektomykorrhizierten Buche verlässlich anwendbar ist, bei der endomykorrhizierten Esche jedoch nicht. Dies wurde an Hand einer angeschlossenen Isotopenmarkierung mit ${ }^{13} \mathrm{C}$ und ${ }^{15} \mathrm{~N}$ bestätigt. Während die vitalen Wurzelspitzen von Buche eine Anreicherung beider Isotope zeigten, trat dies in den trockenen Wurzelspitzen nicht auf und bestätigte dadurch die Anwendbarkeit der Methode der visuellen Ansprache. Bei der Esche wurden beiden Isotope jedoch sowohl in vitalen als auch in trockenen 
Wurzelspitzen angereichert, was bei den trockenen Wurzelspitzen, die als physiologisch inaktiv eingeschätzt wurden, jedoch nicht der Fall hätte sein dürfen.

Die Kohlenstofftranslokation von den Blättern in die Rhziosphäre wurde über einen längeren Betrachtungszeitraum von zwei Vegetationsperioden bei Buche, Winterlinde und Bergahorn in einem Gewächshausexperiment untersucht. Die Frage, ob die Rhizosphäre durch langfristige oder kurzfristige C-Ressourcen von der Pflanze gespeist wird, wurde dabei untersucht. Dabei wurde eine langfristige ${ }^{13} \mathrm{CO}_{2}$ Begasung über zwei Vegetationsperioden mit einer kurzfristigen ${ }^{14} \mathrm{CO}_{2}$ Pulsbegasung kombiniert. Es zeigte sich dabei, dass sowohl langfristiges als auch kurzfristiges $C$ von den Blättern in die Rhizosphäre transportiert wird. Daneben trat eine deutliche Anreicherung von beiden Isotopen in allen Pflanzenorganen über einen kurzen Zeitraum auf, die dann in der folgenden Vegetationsperiode absank. Die Ergebnisse deuten sowohl auf Transport als auch Speicherung von lang- und kurzfristigem $\mathrm{C}$ in allen untersuchten Baumarten hin.

Die vorliegende Arbeit zeigt den Stofftransport von Kohlenstoff und Stickstoff von den Quellen zu den Verbrauchsorten und deutet auf unterschiedliches Verhalten der untersuchten Baumarten hin, wobei ein genereller Unterschied in Bezug auf die Mykorrhizaform nicht eindeutig bewiesen werden konnte. Dazu wären weitere Experimente notwendig. Dies gilt ebenso für die Überprüfung der angewandten Methoden, bei der die Isotopentechnik als sehr zuverlässige Methode angesehen wird, da die Ergebnisse mit denen anderer Untersuchung zum Stofftransport an Hand Isotopentechnik konform gehen. Die angewandte Methodik der visuellen Ansprache von Wurzelspitzen in Bezug auf die Vitalität zeigt auf, dass hier zum einen eine kritische Betrachtung und zum anderen noch Forschungsbedarf an weiteren Baumarten notwendig sind. 


\section{Literatur}

Ellenberg H (1996) Vegetation Mitteleuropas mit den Alpen: in ökologischer, dynamischer und historischer Sicht. Eugen Ulmer Verlag, Stuttgart, 5. Auflage

Finlay R, Soderström B (1992) Mycorrhiza and carbon flow to the soil. In Allen MF: Mycorrhizal functioning- an integrative plant- functional process. Chapman and Hall, New York

Finzi AC, Van Breeman N, Canham CD (1998) Canopy tress soil interactions within temperate forests. Species effects on soil carbon and nitrogen. Ecological Applications 8. 440 - 446

Hobbie JE, Hobbie EA (2006) ${ }^{15} \mathrm{~N}$ in symbiotic fungi and plants estimates nitrogen and carbon flux rates in arctic tundra. Ecology 87: 816 - 822

Lang C, Seven J, Polle A (2011) Host preferences and differential contributions of deciduous tree species mycorrhizal species richness in a mixed Central European forest. Mycorrhiza 21: 297-308

Lovett GM, Rueth H (1999 Soil nitriogen transformations in beech and maple stands along a nitrogen deposition gradient. Ecological Applications 9. 1330 - 1344

Marschner H (1995) Mineral nutrition of higher plants. Academic Press, London

Read DJ \& Perez-Moreno J (2003) Mycorrhizas and nutrient cycling in ecosystems a journey towards relevance? New Phytol 157: 475 - 492

Röhrig E, Bartsch N (1992) Waldbau auf ökologischer Grundlage. Erster Band: Der Wald als Vegetationsform und seine Bedeutung für den Menschen. Parey Verlag, Hamburg \& Berlin, 6. Auflage

Stinglwagner GKF, Haseder IE, Erlbeck R (2005) Das Kosmos Wald- und Forstlexikon. Kosmos Verlag, Stuttgart, 3. Auflage

Tamm CO (1991) Nitrogen in the terrestrial environment. Questions of productivity, vegetional changes and ecosystem stability. Springer Verlag, Berlin 


\section{Summary}

Many indigenous deciduous trees are associated with mycorrhizae and this symbiosis provides nutrients for plants and fungi. The in Europe dominant beech (Fagus sylvatica) is associated with ectomycorrhizas and occurs in Germany both in pure stands or in association with other deciduous trees such as lime, ash and maple on. In the present study the nutrient transfer of two major plant nutrients, carbon and nitrogen, from the plant into the rhizosphere was investigated. Beech was compared with other deciduous trees and the aspect of different mycorrhizae forms on the different tree species was investigated. Different marking experiments were performed by using the technique of isotope as a tracer for the examined substances.

Beech is associated with ectomycorrhiza and was compared in the study with the endomycorrhiza forming ash. The investigations -with respect to Isotope experiments- of the present study were compared with studies done to a far lesser extent on maple and lime to widen the insight into nutrient transfer. By selecting the two tree species the mycorrhiza form was also an important factor. Maple forms endomycorrhiza and lime the ectomycorrhiza. To widen the focus on the two tree species beech and ash in terms of physiological aspects, a vitality investigation was carried out.

To analyze the transport of carbon and nitrogen in the native tree species and their associated mycorrhiza, different labeling experiments were carried out.

In a field experiment the uptake of nitrogen in beech roots both in pure and in mixed stands was examined. The soil had been labeled with ${ }^{15} \mathrm{~N}$ and over a period of 8 days absorption was analyzed. A strong enrichment of ${ }^{15} \mathrm{~N}$ was found in fine roots in both the pure and mixed stands in the beginning with a remaining constant level over the week. The pure stand showed a higher nitrogen enrichment in the fine roots than the mixed stands.

Based on this, a field labeling experiment was conducted, including the ectomycorrhiza forming beech as well as the endomycorrhizal forming ash. The nutrient transfer was investigated within the plants rhizosphere with two different 
mycorrhizal forms. The trees were marked with ${ }^{15} \mathrm{~N}$ for three days via theirs leaves and subsequently with ${ }^{13} \mathrm{CO}_{2}$ for five hours. The transport of $\mathrm{C}$ and $\mathrm{N}$ was analyzed over a period of 60 days. It turned out that the endomycorrhiza forming Ash accumulated both more $\mathrm{C}$ and more $\mathrm{N}$ in their fine roots and root tips than the ectomykorrhiza forming beech.

Looking at the physiology the nutrient transport in the plant is related to the vitality of the roots, especially the fine roots. This should be tested in an additional investigation. Therefore the roots of the two studied tree species beech and ash were visually checked. The root tips were further checked with a TTC tests on their vitality status. From the comparison of vital and dry root tips it turned out that the method of visual checking root tips is reliable for the ectomycorrhiza forming beech, but not for the endomycorrhiza forming ash. An labelling experiment with $\mathrm{C}$ and $\mathrm{N}$ was installed to analyse nutrient accumulation in vital and dry root tips of both species. In case of beech there was an accumulation of $\mathrm{C}$ and $\mathrm{N}$ just in vital root tips. In case of ash, it was shown that there was an accumulation of $\mathrm{C}$ and $\mathrm{N}$ in vital and dry root tips. This suggest that method of visually characterization of vital and dry root tips is suitable for beech, but not for ash.

The question of whether the rhizosphere is fed by long-term or short-term carbon resources of the plant was investigated in a greenhouse experiment with sycamore maple, beech and basswood. Long-term ${ }^{13} \mathrm{CO}_{2}$ fumigation over two growing seasons was combined with a short-term ${ }^{14} \mathrm{CO}_{2}$ pulse labeling. It was found in all three tree species that both long-term and short-term $C$ is transported from the leaves to the rhizosphere. In addition, a significant accumulation of both isotopes occurred in all plant organs in a short time, which fell in the following growing season. The results suggest both transport and storage of long- and short-term $C$ in all studied species.

In summary, in the present work it was shown that nutrient transfer was detected from the leaves to the roots in all the studied native deciduous tree species and some differences in the transport strategies of tree species occur. 


\section{Anhang}

Kapitel $5,{ }^{13} \mathrm{C}$ - und $\partial^{14} \mathrm{C}$ - Doppelmarkierung zur Analyse des Transportes von Kohlenstoff in die Rhizosphäre einheimischer Laubbaumarten"

\section{Biomasse}

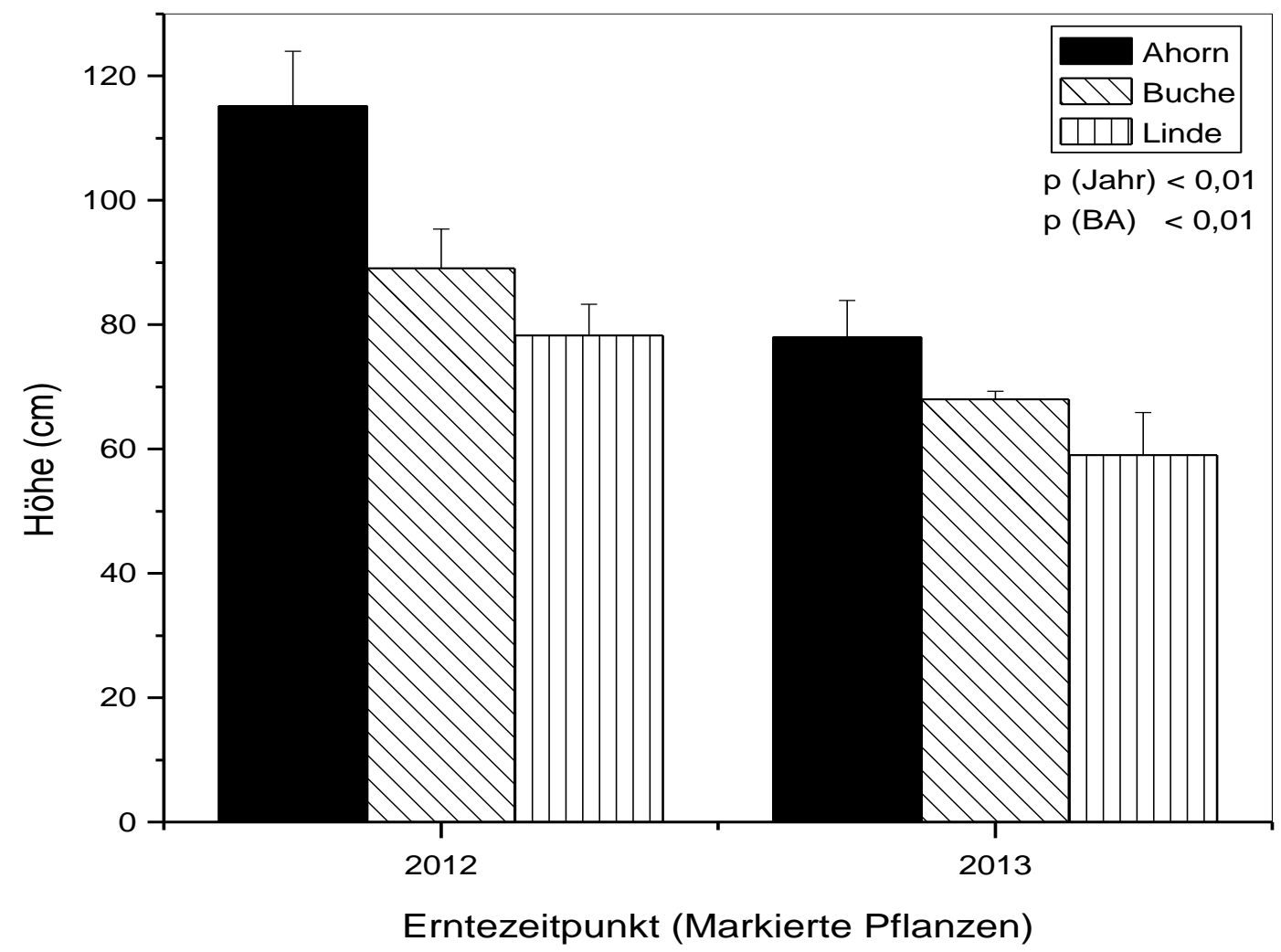

Abbildung 5.3.1: Pflanzenhöhe der Baumarten in den beiden ${ }^{13} \mathrm{C}$-begasten Vegetationsperioden 2012 und 2013, entsprechend den Erntezeitpunkt von 1 Tag und 1 Woche (2012) und 1 Jahr nach der ${ }^{14} \mathrm{C}$ Markierung (2013); Pflanzenhöhen der Kontrollen nicht vorhanden; Daten zeigen MW \pm SE; $n=3-10$, BA = Baumart, Two-Way-ANOVA mit den Faktoren Zeit $(2012,2013)$ und Baumart (Ahorn, Buche, Linde) 


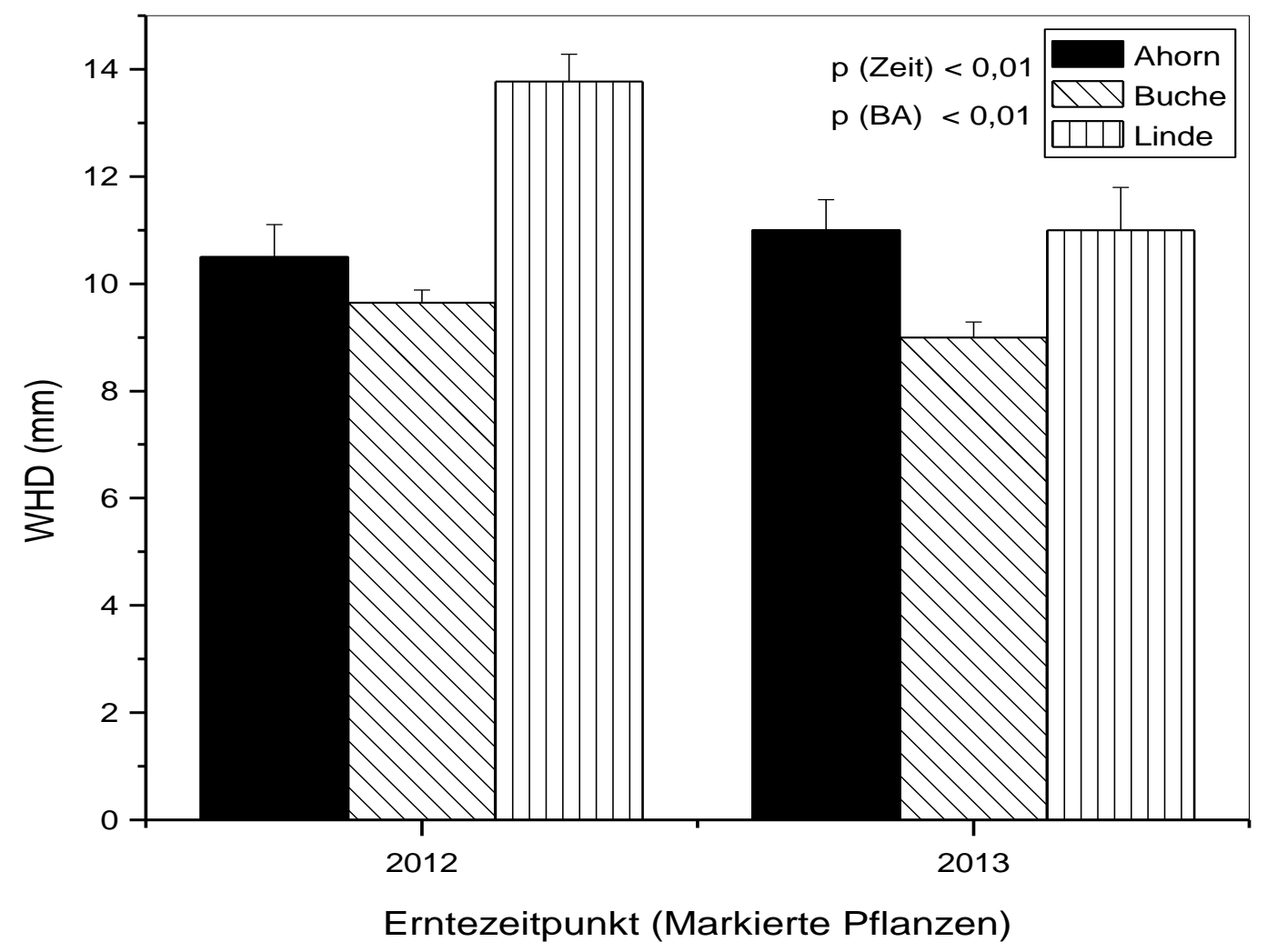

Abbildung 5.3.2: Wurzelhalsdurchmesser (WHD) der Baumarten in den beiden ${ }^{13} \mathrm{C}$-begasten Vegetationsperioden 2012 und 2013, entsprechend den Erntezeitpunkt von 1 Tag und 1 Woche (2012) und 1 Jahr nach der ${ }^{14} \mathrm{C}$-Markierung (2013);WHD der Kontrollen nicht vorhanden; Daten zeigen MW \pm SE; $n=3-10, B A=$ Baumart, Two-Way-ANOVA mit den Faktoren Zeit $(2012,2013)$ und Baumart (Ahorn, Buche, Linde) 


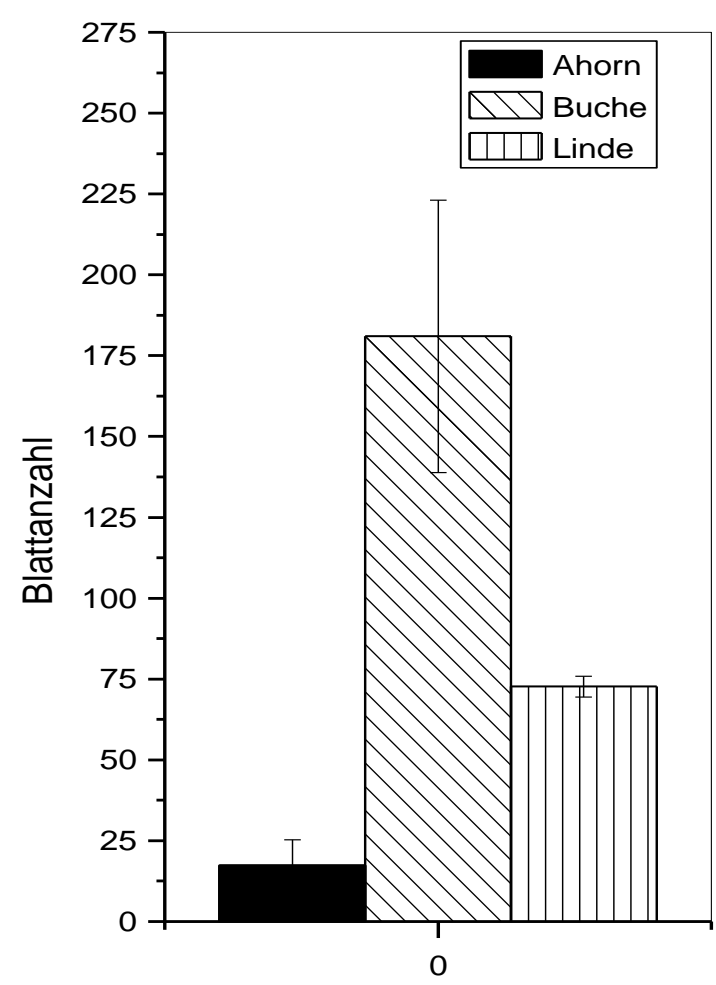

Erntezeitpunkt (Kontrollen)

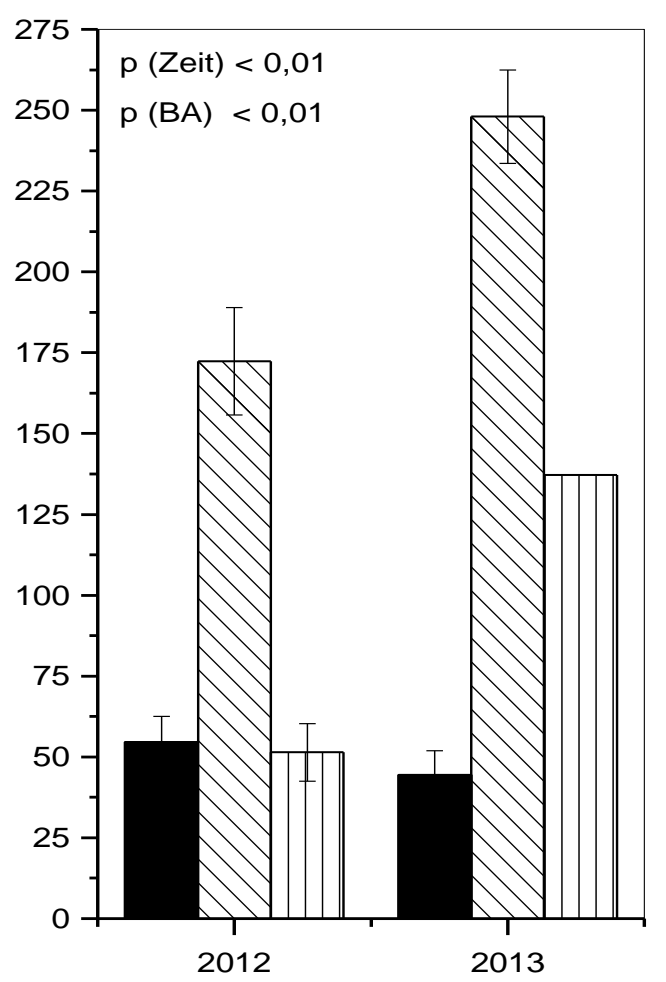

Erntezeitpunkt (Markierte Pflanzen)

Abbildung 5.3.3: Blattanzahl ( $\mathrm{n}$ ) der Baumarten in den Kontrollen (0) und beiden ${ }^{13} \mathrm{C}$-begasten Vegetationsperioden 2012 und 2013, entsprechend den Erntezeitpunkt von 1 Tag und 1 Woche (2012) und 1 Jahr nach der ${ }^{14} \mathrm{C}$-Markierung (2013); Daten zeigen MW \pm SE; $n=3-10$, BA = Baumart; Two- Way-ANOVA mit den Faktoren Zeit $(0,2012,2013)$ und Baumart (Ahorn, Buche, Linde) 


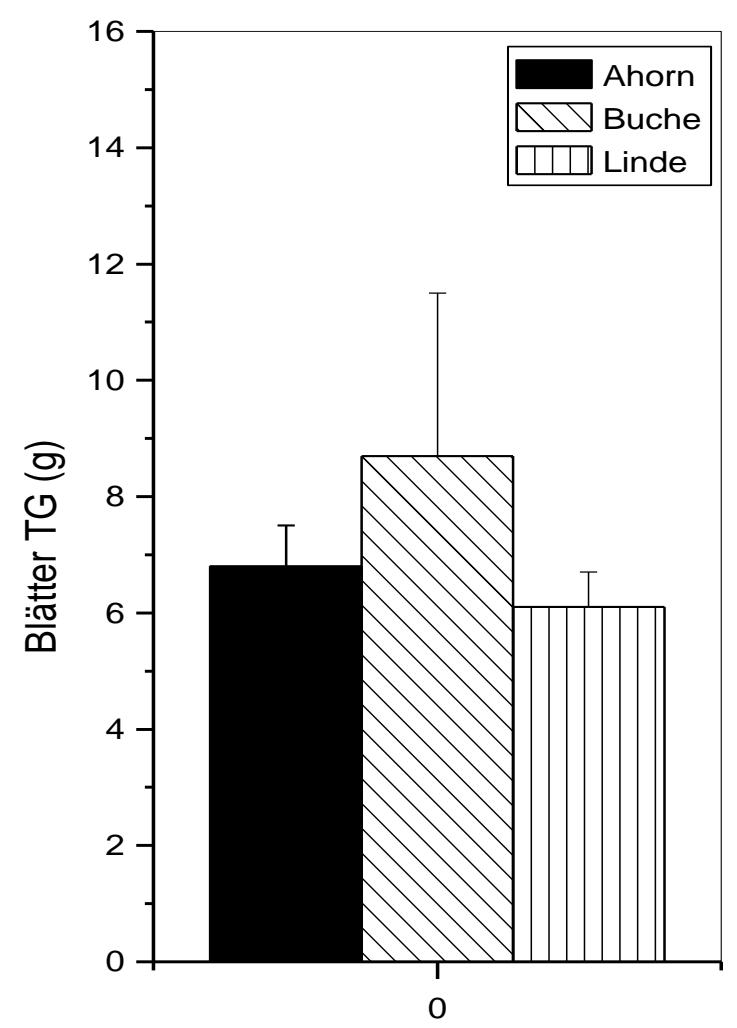

Kontrolle (Erntezeitpunkt 0)

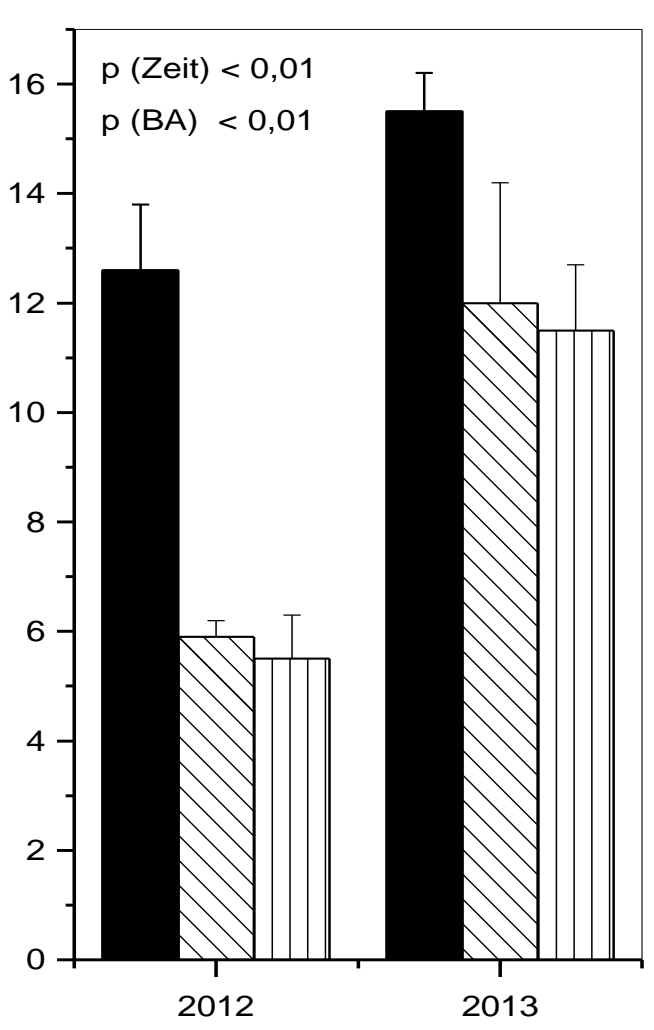

Erntezeitpunkt (Markierte Pflanzen)

Abbildung 5.3.4: Trockenmasse (TG) der Blätter in den Kontrollen und beiden ${ }^{13} \mathrm{C}$-begasten Vegetationsperioden 2012 und 2013, entsprechend den Erntezeitpunkt von 1 Tag und 1 Woche (2012) und 1 Jahr nach der ${ }^{14} \mathrm{C}$-Markierung (2013); Daten zeigen MW $\pm \mathrm{SE} ; \mathrm{n}=3-10$, BA = Baumart; Two- Way-ANOVA mit den Faktoren Zeit $(0,2012,2013)$ und Baumart (Ahorn, Buche, Linde) 


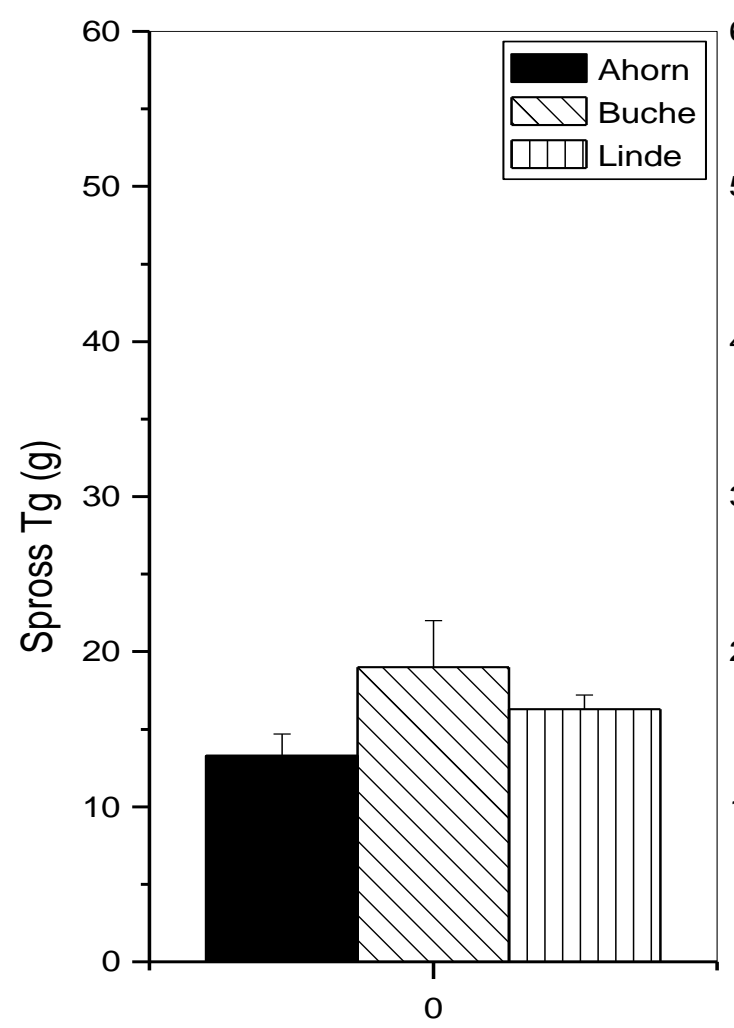

Erntezeitpunkt (Kontrollen)

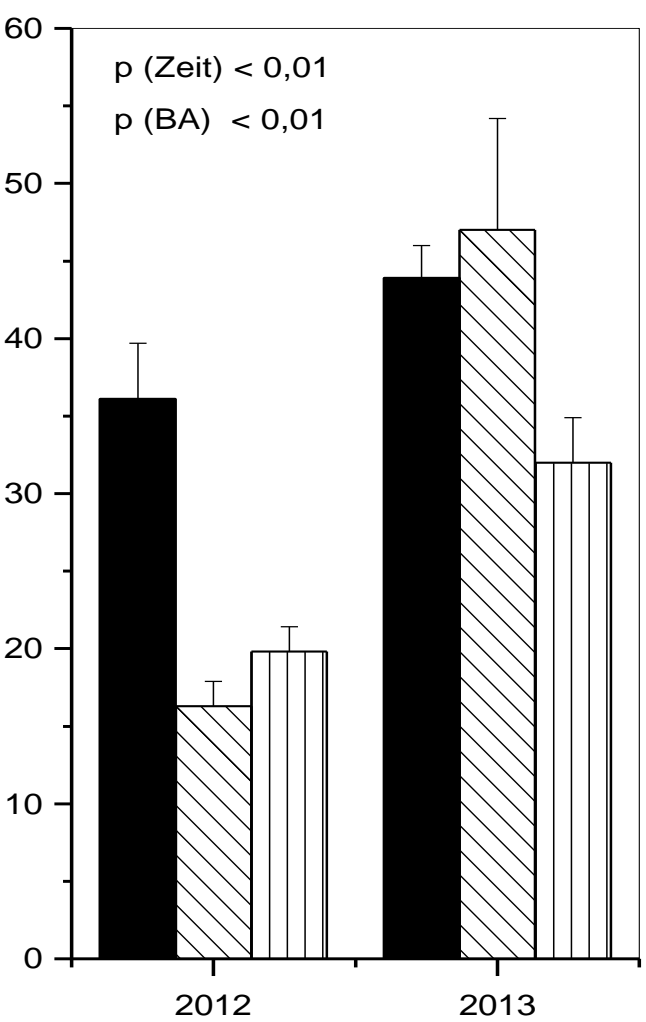

Erntezeitpunkt (Markierte Pflanzen)

Abbildung 5.3.5: Trockenmasse (TG) des Sprosses in den Kontrollen und beiden ${ }^{13} \mathrm{C}$-begasten Vegetationsperioden 2012 und 2013, entsprechend den Erntezeitpunkt von 1 Tag und 1 Woche (2012) und 1 Jahr nach der ${ }^{14} \mathrm{C}$-Markierung (2013); Daten zeigen MW $\pm \mathrm{SE} ; \mathrm{n}=3-10$, BA = Baumart; Two- Way-ANOVA mit den Faktoren Zeit $(0,2012,2013)$ und Baumart (Ahorn, Buche, Linde) 


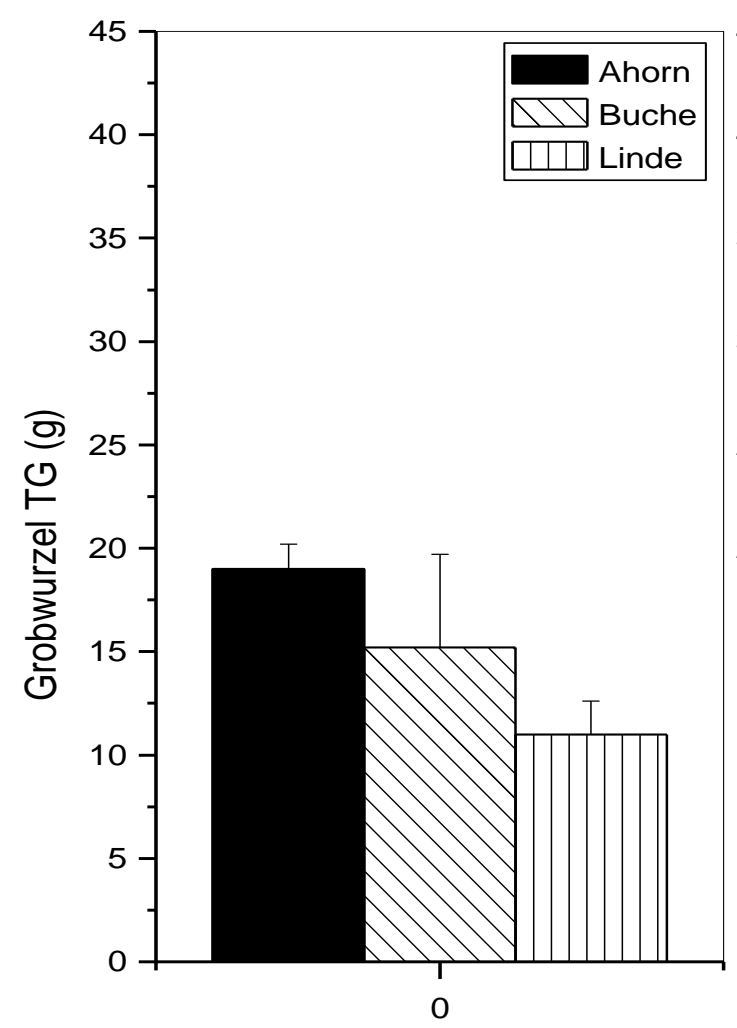

Erntezeitpunkt (Kontrollen)

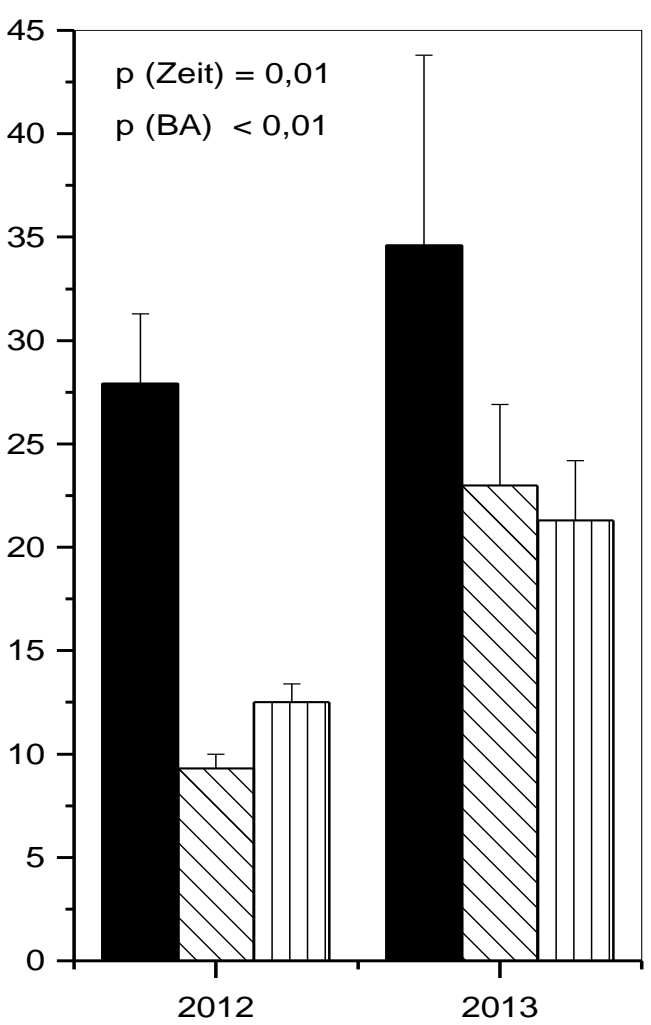

Erntezeitpunkt (markierte Pflanzen)

Abbildung 5.3.6: Trockenmasse (TG) der Grobwurzeln in den Kontrollen und beiden ${ }^{13} \mathrm{C}$-begasten Vegetationsperioden 2012 und 2013, entsprechend den Erntezeitpunkt von 1 Tag und 1 Woche (2012) und 1 Jahr nach der ${ }^{14} \mathrm{C}$-Markierung (2013); Daten zeigen MW $\pm \mathrm{SE} ; \mathrm{n}=3-10$, BA = Baumart; Two- Way-ANOVA mit den Faktoren Zeit $(0,2012,2013)$ und Baumart (Ahorn, Buche, Linde) 


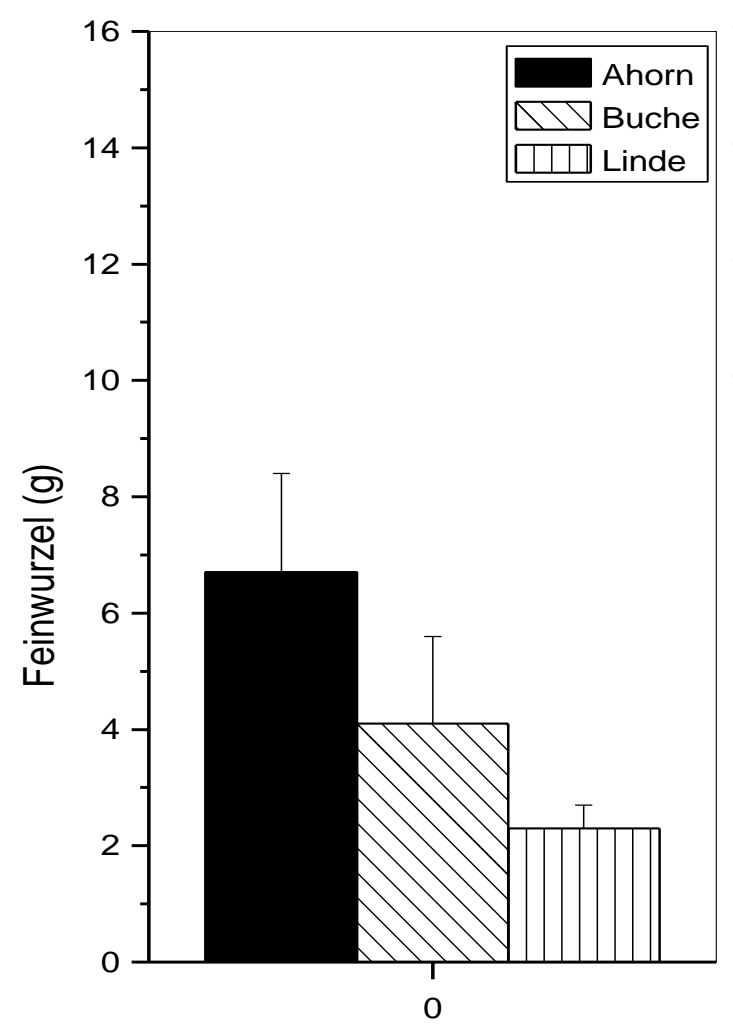

Erntezeitpunkt (Kontrollen)

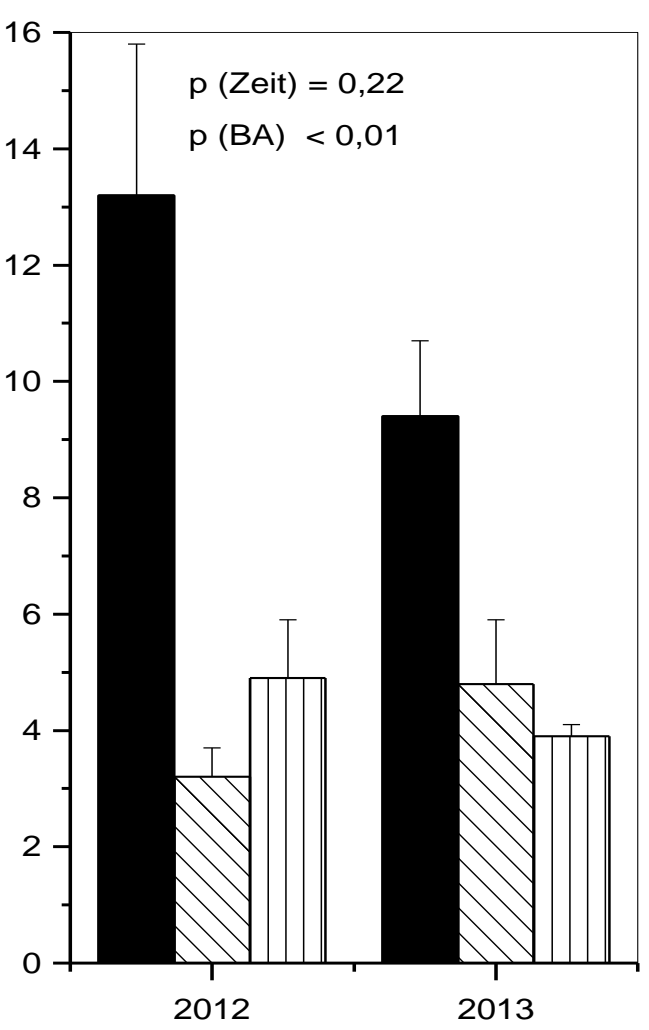

Erntezeitpunkt (Markierte Pflanzen)

Abbildung 5.3.7: Trockenmasse (TG) der Feinwurzeln in den Kontrollen und beiden ${ }^{13} \mathrm{C}$-begasten Vegetationsperioden 2012 und 2013, entsprechend den Erntezeitpunkt von 1 Tag und 1 Woche (2012) und 1 Jahr nach der ${ }^{14} \mathrm{C}$-Markierung (2013); Daten zeigen MW $\pm \mathrm{SE} ; \mathrm{n}=3-10$, BA = Baumart; Two- Way-ANOVA mit den Faktoren Zeit $(0,2012,2013)$ und Baumart (Ahorn, Buche, Linde) 
Lebenslauf

Michaela Rath

geboren am 12.Oktober 1982 in Kassel

Konfession: evangelisch

Nationalität: deutsch

Schulischer und wissenschaftlicher Werdegang

1989- 1993

Ludwig-Emil-Grimm Grundschule, Fuldatal

1993- 1999

IGS Gesamtschule Fuldatal

Realschulabschluss

$1999-2003$

Albert-Schweitzer-Gymnasium Kassel

Abitur

2003- 2008

Bachelorstudium der Forstwissenschaften und Waldökologie an der Georg-August-Universität Göttingen

B. Sc. Forstwissenschaften und Waldökologie

09/2004 - 12/2004 Betriebspraktikum im Hessischen Forstamt Witzen hausen

2008

Bachelorarbeit „Physiologischer Einfluss von Wurzelstress an

Pappel“ am Büsgen-Institut der Georg-August- Universität Göttingen, Abt. Forstbotanik, Prof. Andrea Polle

2008 - 2011 Masterstudium der Forstwissenschaften und Waldökologie an der Georg-August-Universität Göttingen

M. Sc. Forstwissenschaften und Waldökologie

2011

Masterarbeit „Einfluss von Trockenheit auf die Diversität der Mykorrhizagesellschaft an jungen Buchen“ am Büsgen-Institut der Georg-August- Universität Göttingen, Abt. Forstbotanik, Prof. Andrea Polle

2011 - heute Promotion im Graduiertenkolleg 1086 „Die Bedeutung der Bio- 
Diversität für Stoffkreisläufe und biotische Interaktionen in temperaten Laubwäldern“ über die Bedeutung der Mykorrhiza für die Nährstoffversorgung einheimischer Laubbaumarten, Prof. Andrea Polle

Hiermit erkläre ich, dass ich die vorliegende Arbeit selbständig und nur mit den erlaubten Hilfsmitteln angefertigt habe.

Michaela Rath

Göttingen, den 
Die vorliegende Arbeit wurde im Rahmen des DFG-Graduiertenkolleg 1086 „Die Bedeutung der Biodiversität für Stoffkreisläufe und biotische Interaktionen in temperaten Laubwäldern“, finanziert durch die Deutsche Forschungsgemeinschaft, angefertigt.

Am Ende dieser langen Reise danke ich vielen Menschen, die an meiner Seite gestanden und mich begleitet haben - ohne die, diese Dissertation nicht entstanden wäre.

Ich danke Frau Prof. Andrea Polle neben der vertrauensvollen Übertragung dieses Forschungsthemas für ihre Betreuung meines wissenschaftlichen Werdegangs seit meiner Bachelorarbeit. In den nunmehr sieben Jahren, in denen ich an ihrem Lehrstuhl studiert, als Hilfskraft gearbeitet, geforscht und gelehrt habe, habe ich unermesslich wertvolle Erfahrungen gemacht und bin sehr dankbar dafür.

Meinen Kollegen in der Forstbotanik, die mir im Laufe der letzten Jahre ans Herz gewachsen sind, werde ich auf ewig dankbar sein. An chronologisch erster Stelle steht dabei Frau Dr. Christa Lang, die mich seit meinem ersten Hiwijob bis zu ihrem Verlassen der Forstbotanik „unter ihre Fittiche“ genommen hat und mich immer (ohne wenn und aber) großartig betreut hat. Ohne sie wäre ich nicht auf die Idee gekommen, eine Doktorarbeit überhaupt zu beginnen. Christine Kettner, Giesbert Langer-Kettner, Bernd Kopka, Gabriele Lehmann und Marianne Smiatacz begleiten mich ebenfalls seit meinen Anfängen in der Forstbotanik und sie sind mir so sehr an mein Herz gewachsen, dass sich meine Dankbarkeit nur schwer in Worte fassen lässt. An den schweren Tagen mit Verzweiflung und mit dem einzigen Wunsch „das alles einfach aus dem Fenster zu schmeißen und morgen nicht mehr wiederzukommen“ haben sie mich immer wieder aufgefangen und ohne sie hätte ich diese Arbeit nicht zu Ende gebracht.

Meinen Kollegen Kristina Schröter, Bettina Otto, Dennis Janz, Martin Leberecht, Josie Sahner und Katharina Volmer danke ich, dass ich den Promotionsmarathon nicht allein laufen musste. Sie und ihre Aufmunterungen, Ratschläge und Diskussionen - beruflich als auch privat - haben meine Arbeit immer wieder bereichert. Ich danke auch Silke Ammerschubert, mit der ich viel Spaß in unseren 
gemeinsamen Lehrveranstaltungen hatte und mit der ich immer wieder über das all umfassende Sein diskutieren kann.

Ich danke weiterhin dem gesamten Team der Forstbotanik - es gibt keinen den ich ausschließen will. Und auch meinen Kollegen aus dem Graduiertenkolleg Sarah Zieger und Ronny Thoms, die mit mir im Freiland "gewütet“ haben.

Mein größter Dank gilt meiner Familie, ohne die diese Arbeit nicht möglich gewesen wäre. Ich danke meinen Eltern Klaus und Regina - diese Arbeit ist „fürr di“-, dass sie zu jedem Moment und ohne jeden Zweifel bei mir waren und an mich geglaubt haben. Wie oft habt ihr mich aus dem Tal der Tränen rausgeholt? Ohne euch hätte ich es nicht geschafft, „nicht aufzugeben“. 